
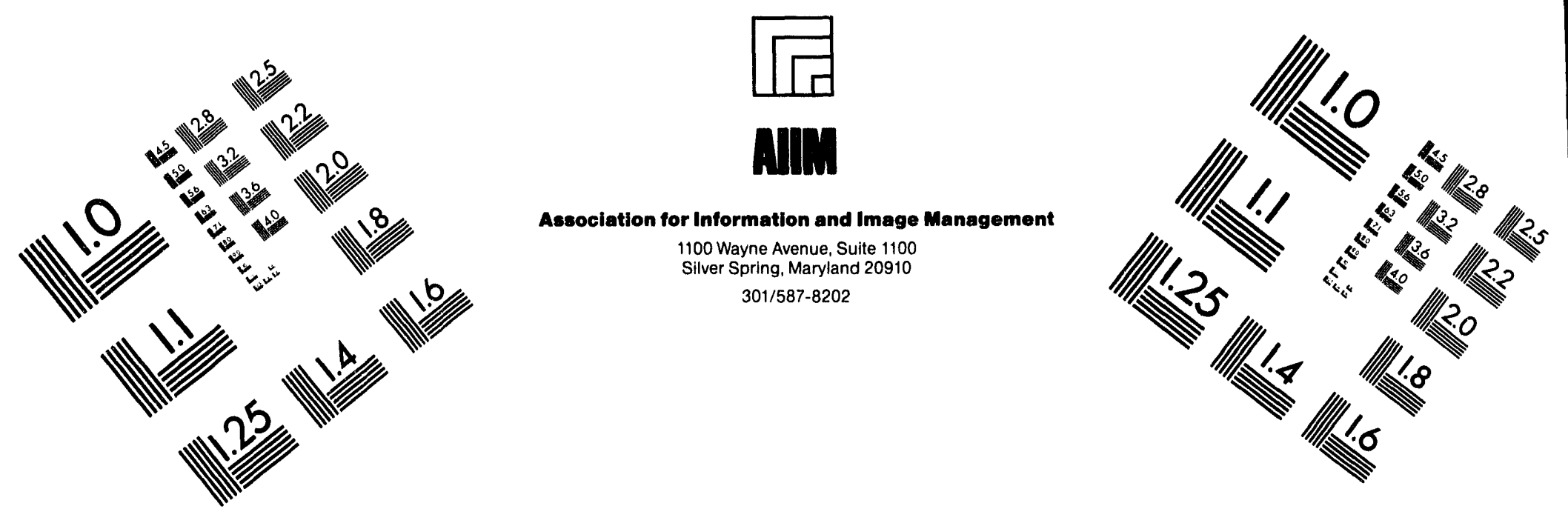

\title{
Centimeter
}

$\begin{array}{llllllllllllllll}1 & 2 & 3 & 4 & 5 & 6 & 7 & 8 & 9 & 10 & 11 & 12 & 13 & 14 & 15 & \mathrm{~mm}\end{array}$ 1 Inches
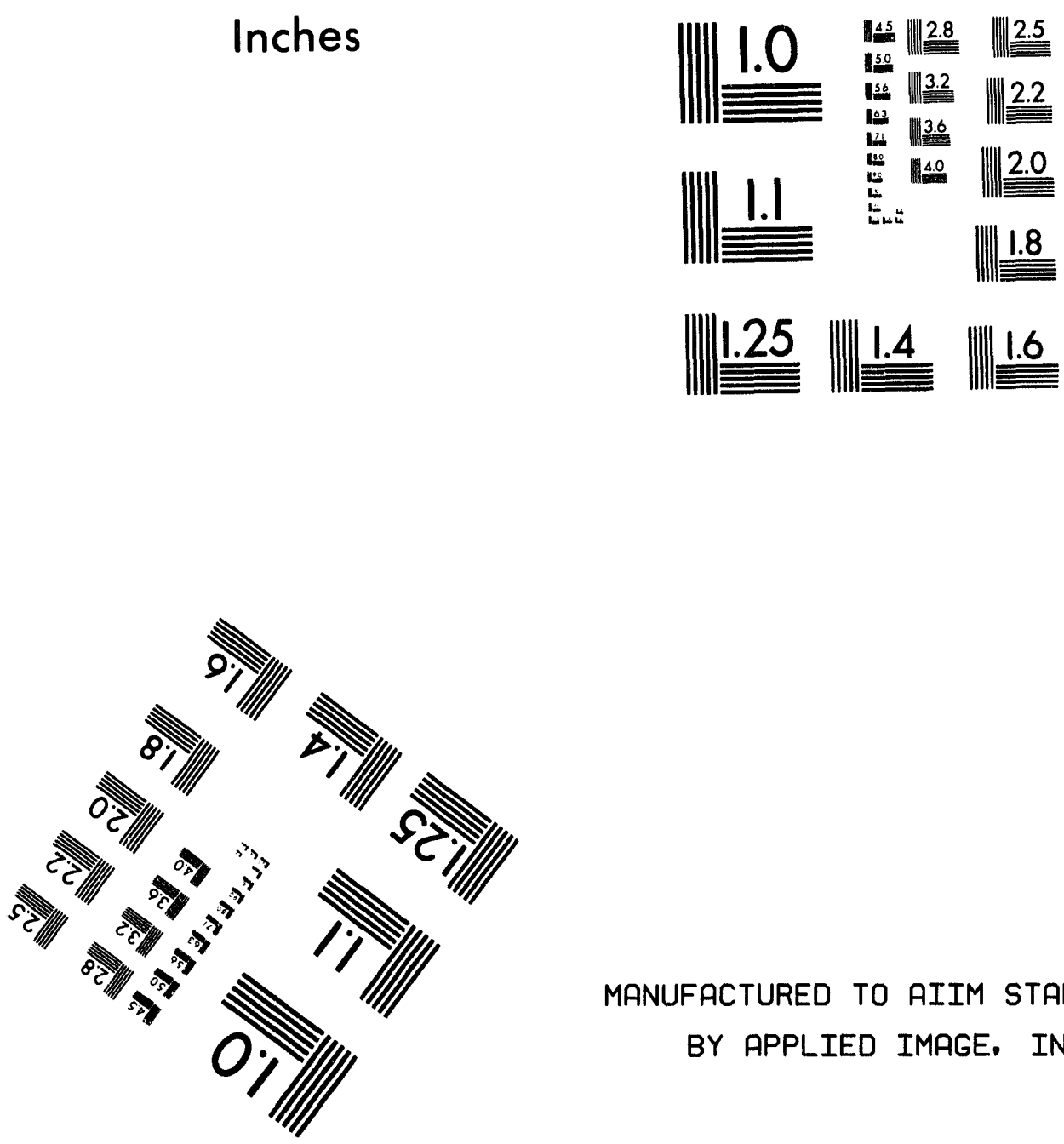

MANUFACTURED TO AIIM STANDARDS BY APPLIED IMAGE, INC.

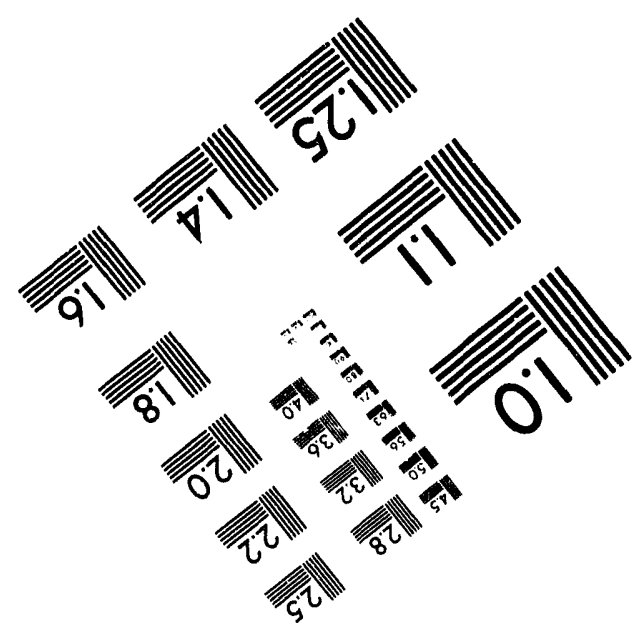



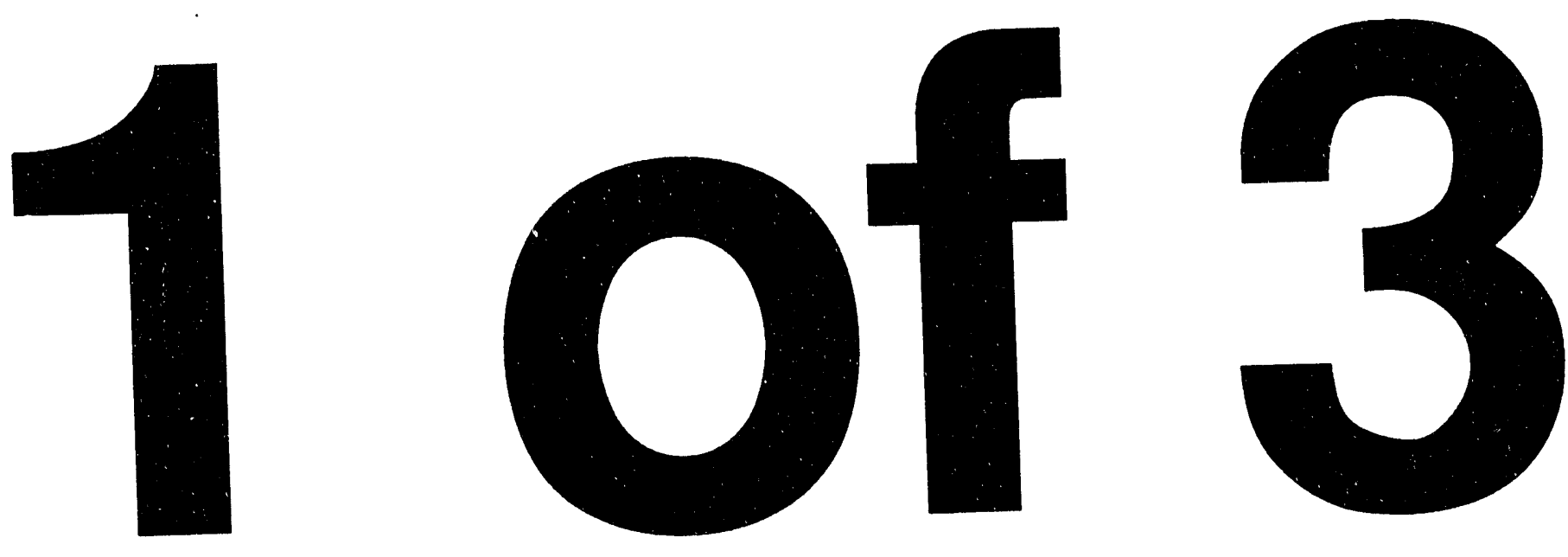


\section{Report on Hanford Site Land Disposal Restrictions for Mixed Waste}

D. Black

Date Published

April 1994 


\section{LEGAL DISCLAIMER}

This report was prepared as an account of work sponsored by an agency of the United States Government. Neither the United States Government nor any agency thereof, nor any of their employees, nor any of their contractors, subcontractors or their employees, makes any warranty, express or implied, or assumes any legal liability or responsibility for the accuracy, completeness, or any third party's use or the results of such use of any information, apparatus, product, or process disclosed, or represents that its use would not infringe privately owned rights. Reierence herein to any specific commercial product, process, or service by trade name, trademark, manufacturer, or otherwise, does not necessarily constitute or imply its endorsement, recommendation, or favoring by the United States Government or any agency thereof or its contractors or subcontractors. The views and opinions of authors expressed herein do not necessarily state or reflect those of the United States Government or any agency thereof.

This report has been reproduced from the best available copy. Available in paper copy and microfiche.

Available to the U.S. Department of Energy

and its contractors from

Office of Scientific and Technical Information

P.0. Box 62

Oak Ridge, TN 37831

(615) $576-8401$

Available to the public from the U.S. Department of Commerce National Technical Information Service

5285 Port Royal Road

Springfield, VA 22161

(703) 487.4650

Printed in the United States of Amorica

DISCI-M-1.CHP (1-91) 


\section{EXECUTIVE SUMMARY}

This report was submitted to meet the requirements of Hanford Federal Facility Agreement and Consent Order' Milestone M-26-01D.

The U.S. Department of Energy, its predecessors, and contractors at the Hanford Site were involved in the production and purification of nuclear defense materials from the early 1940s to the late 1980s. These production activities have generated large quantities of 1 iquid and solid radioactive mixed waste. This waste ic slibject to regulation under authority of both the Resource Conservation and Recovery Act of $1976^{2}$ and Atomic Energy Act of $1954 .^{3}$ This report covers mixed waste only. Hazardous waste that is not contaminated with radionuclides is not addressed in this report.

The Washington State Department of Ecology, U.S. Environmental Protection Agency, and U.S. Department of Energy have entered into an agreement, the Hanford Federal Facility Agreement and Consent Order ${ }^{\dagger}$ (commonly referred to as the Tri-Party Agreement) to bring the Hanford Site operations into compliance with dangerous waste regulations. The Tri-Party Agreement required development of the original land disposal restrictions (LDR) plan and its annual updates to comply with LDR requirements for radioactive mixed waste. This report is the fourth update of the plan first issued in 1990.

Tri-Party Agreement negotiations completed in 1993 and approved in January 1994 changed and added many new milestones. Most of the changes were related to the Tank Waste Remediation System and these changes are incorporated into this report.

The Tri-Party Agreement requires, and the baseline plan and annual update reports provide, the information that follows.

- Waste Characterization Information--Provides information regarding the characterizing of each LDR mixed waste. The sampling and analysis methods and protocols, past characterization results, and a schedule for providing the characterization information, where available, are discussed.

- Storage Data--Identifies and describes the mixed waste at the Hanford Site, including the following: the Resource Conservation and Recovery Act of 1976 dangerous waste code(s), process information necessary to identify the waste and make LDR determinations, quantities stored, generation rates, location and

${ }^{1}$ Ecology, EPA, and DOE, 1992, Hanford Federal Facility Agreement and Consent Order, Vol. 1 and 2, as updated by the fourth amendment dated January 25, 1994, Washington State Department of Ecology, U.S. Environmental Proteçtion Agency, and U.S: Department of Energy, 01 ympia, Washington.

Resource Conservation and Recovery Act of 1976, as amended, 42 USC 6901, et seg.

${ }^{5}$ Atomic Energy Act of 1954, as amended, 42 USC 2011. 
method of storage, an assessment of storage unit compliance status, storage capacity, and the bases and assumptions used in making the estimates.

- Treatment Information--Identifies the current treatment processes, plans, and schedules for developing treatment technologies that meet LDR treatment standards. Also included are discussions of treatment alternatives and accelerated treatment.

- Waste Reduction Information--Identifies methods for reducing the generation of land disposal restricted waste. Includes treatment methods and process changes made or planned to reduce the generation of LDR waste, methods to minimize the volume of LDR waste, and methods to minimize the toxicity of newly generated waste.

- Schedule--Provides schedules depicting the events necessary to achieve compliance with LDR requirements, including variances, exemptions, or time extensions necessary to achieve LDRs compliance.

- Progress--Identifies progress made in achieving compliance since the previous LDRs report.

A Tri-Party Agreement change request for the LDR report milestone was approved in 1992. This change request consolidated another LDR report, Milestone M-25-00, that emphasized LDR treatment alternatives. Therefore, this LDR report now includes increased discussion of treatment alternatives.

The Hanford Site waste primarily resulted from defense materials production. Usable defense materials were separated from fission products waste through precipitation and solvent extraction processes. Large quantities of liquid waste resulted from these separation processes and were stored in underground single-shell tanks (SST) and double-shell tanks (DST). Additional waste volumes resulted from nuclear fuel fabrication activities, process laboratories activities, decontamination and cleaning of equipment and building structures, closure of process and storage units, and research and development activities such as Fast Flux Test Facility operation.

Projected generation rates, after waste reduction, range from approximately 26,749 cubic meters per year to approximately 46,868 cubic meters per year. These rates are in the years 1998 and 1995, respectively.

The waste addressed in this report includes mixed waste (i.e., hazardous waste that contains radionuclides) designated as characteristic dangerous waste; designated as toxic, carcinogenic, and persistent by the Washington State criteria; and 1isted waste because it contains small amounts of spent solvents and discarded pure chemical products. The waste consists of liquid, sludges, hard crystalline material (salt cake), and materials such as contaminated equipment, paper, and rags. Much is already known about the waste characteristics from process information and sampling and analysis programs. Action schedules have been developed to further characterize the waste. 
The waste currently is stored in underground SSTs and DSTs, in containers placed in storage units such as the Hanford Solid Waste Operations Complex, caissons, and retrievable storage units. A surface impoundment, the Liquid Effluent Retention Facility, has been constructed to store large quantities of waste that contain radionuclide concentrations low enough to allow surface storage. The waste will be removed from these storage units, treated to meet LDR standards, and sent to final disposal in accordance with schedules established in Tri-Party Agreement milestones $M-17$ and M-26.

Total Hanford Site storage capacity for LDR waste is approximately 574,000 cubic meters. About 366,000 cubic meters of this capacity is in units such as SSTs that no longer actively receive waste. Approximately 243,830 cubic meters of waste are currentiy in storage. The DSTs currently available are essentially filled to capacity. To alleviate the space shortage, up to six new DSTs are planned. The Liquid Effluent Retention Facility basins dedicated to 242-A Evaporator process condensate will be filled in mid 1995 and the storage space currently available at the Central Waste Complex is anticipated to be filled in 1999; however, additional buildings will be constructed as required to store waste generated in the future.

The waste treatment processes for these wastes include the current treatment processes to reduce corrosion of storage tanks and planned treatment processes to reduce waste toxicity and immobilize waste constituents. Current waste treatment consists of $\mathrm{pH}$ adjustment and corrosion inhibitors and using absorbents and solidifying agents. Planned waste treatment processes include development of neutralization and toxic constituent destruction processes (corrosivity neutralization processes), development of waste separation and pretreatment processes (Waste Receiving and Processing Facility Module 2), separation of tank waste (pretreatment) into a low-level waste fraction and a high-level fraction which will both be vitrified, and development of an organic destruction process (the Treated Effluent Disposal Facility).

The Hanford Site developed a sitewide waste minimization plan that sets minimization goals and establishes processes for measuring progress toward these goals. Each plant or process has a plan to implement the sitewide goals.

The continued storage of land disposal restricted wastes until sufficient treatment and disposal capacity is available was negotiated as part of the Tri-Party Agreement. Schedules to implement the dangerous waste management compliance activities until treatment capacity is available are described in the Tri-Party Agreement. Any newly identified compliance actions will be scheduled in accordance with procedures established in the agreement. 


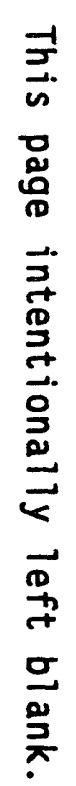




\section{ACRONYMS AND ABBREVIATIONS}

ALARA

BDAT

CAW

CCW

CCWE

CERCLA

CFR

CUU

CWC

CXP

D\&AL

$D \& D$

LBP

DOE

DOE-HQ

DSS

DSSF

DST

Ecology

EIS

EPA

ETF

FFCA

FFTF

FR

FY

GTF

HEPA

HLW

HOC

HSW

HWVP

IEMC

INEL

LDR

LERF

LLBG

LLW

LSA

LSW

MBP

NA

National Report

NCAW

NCRW

NEPA

ORNL

PCB

PFP as low as reasonably achievable

best demonstrated avajlable technology

CA Column Aqueous Waste Stream

constituent concentrations in waste

constituent concentration in the waste extract

Comprehensive Environmental Response Compensation

and Liability Act of 1980

Code of Federal Regulations

CU Column Aqueous Waste Stream

Central Waste Complex

CX Column Aqueous Waste Stream

Development and Analytical Laboratories

Deactivation and Decommissioning

Di-Butyl Phosphate

U.S. Department of Energy

U.S. Department of Energy-Headquarters

Double-Shell Slurry

Double-Shell Slurry Feed

double-shell tank

Washington State Department of Ecology

Environmental Impact Statement

U.S. Environmental Protection Agency

Effluent Treatment Facility

Federal Facilities Compliance Act

Fast Fiux Test Facility

Federal Register

fiscal year

Grout Treatment Facility

high-efficiency particulate air (filter)

high-level waste

halogenated organic carbon

High-Salt Waste

Hanford Waste Vitrification Plant

Interim Examination and Maintenance Cell

Idaho National Engineering Laboratory

land disposal restriction

Liquid Effluent Retention Facility

low-level burial grounds

low-level waste

low specific activity

Low-Salt Waste

Mono-Butyl Phosphate

not applicable

National Report on Prohibited Wastes and Treatment

Options (DOE 1990)

neutralized current acid waste

neutralized cladding removal waste

National Environmental Policy Act of 1969

Oak Ridge National Laboratory

polych? orinated biphenyl

Plutonium Finishing Plant 


\section{ACRONYMS AND ABBREVIATIONS (cont)}

PNL

PRF

PUREX

RCRA

$\mathrm{RL}$

RMC

RMW

SALDS

SRS

SST

TBD

TBP

TCLP

TOC

TOX

Tri-Party Agreement

TRU

TRUEX

TRUPACT

TRUSAF

TSCA

WAC

WDOE

WERF

WIPP

WRAP
Pacific Northwest Laboratory

Plutonium Reclamation Facility

Plutonium-Uranium Extraction (Plant)

Resource Conservation and Recovery Act of 1976

U.S. Department of Energy, Richland Operations Office Remote Mechanical "C" Line

radioactive mixed waste

state-approved land disposal structure

Savannah River Site

single-shell tank

to be determined

Tri-Butyl Phosphate

toxic characteristic leach procedure

Total Organic Carbon

total organic halide

Hanford Federal Facility Agreement and Consent Order transuranic

transuranic extraction

transuranic package transporter

Transuranic Waste Storage and Assay Facility

Toxic Substances Control Act of 1976

Washington Administrative Code

Washington State Department of Energy

Waste Experimental Reduction Facility

Waste Isolation Pilot Plant

Waste Receiving and Processing (Facility) 


\section{CONTENTS}

1.0 INTRODUCTION . . . . . . . . . . . . . . . . . . . . . 1-1

1.1 BACKGROUND AND PURPOSE .................. . . . 1-2

1.2 ASSUMPTIONS ......................... . . . . . $1-3$

1.3 SCHEDULE AND MECHANICS OF PLAN UPDATE . . . . . . . . . . . 1-5

1.4 MILESTONE PLANNING PROCESS . . . . . . . . . . . . . 1-5

1.5 ACTIVITIES AND ACHIEVEMENTS ............... . . . . . .

2.0 SITF SUIMARY . . . . . . . . . . . . . . . . . . . 2-1

2.1 WASTE GENERATION .................... 2-1

2.2 WASTE CHARACTERIZATION ................... 2-2

2.3 WASTE STORAGE ..................... 2-3

2.4 WASTE TREATMENT .................... 2-4

2.4.1 Double-She11 Tank Waste... . . . . . . . . 2-6

2.4.2 PUREX Plant Aging Waste ............... 2-7

2.4.3 Single-Shell Tank Waste .............. 2-7

2.4.4 242-A Evaporator Process Condensate . . . . . . . . 2-7

2.4.5 4843 Sodium Storage Facility Waste . . . . . . . . 2-7

2.4.6 PUREX PIant Ammonia Scrubber Waste . . . . . . . . 2-7

2.4.7 PUREX PIant Process Condensate . . . . . . . . . . 2-8

2.4.8 Hexone Waste . . . . . . . . . . . . . . . . 2-8

2.4.9 183-H Solar Evaporation Basins Waste . . . . . . . 2-8

2.4.10 PUREX Storage Tunnels 1 and 2 Waste (Lead, Mercury, and Silver) and PUREX Containment Building (Lead and Cadmium) .................. 2-9

2.4.11 Central Waste Complex Stored Low-Level,

Transuranic, and Polychlorinated Biphenyl Waste;

TRUSAF Stored Waste; and Retrievably Stored Low-Level, Transuranic, and Polychiorinated

Biphenyl Waste.................. 2-9

2.4.12 303-K Stored Waste................ 2-9

2.5 WASTE MINIMIZATION .................... . . 2-10

2.5.1 Waste Minimization Program Elements . . . . . . . 2-10

2.5.2 Program Objectives .. . . . . . . . . . . . 2-11

2.5.3 Facility-Specific Waste Minimization ....... . 2-12

2.6 VARIANCES, EXEMPTIONS, AND TIME EXTENSIONS ........

3.0 INDIVIDUAL WASTE STREAM INFORMATION ............... . . 3-1

3.1 DOUBLE-SHELL TANK WASTE ................. . . 3-1

3.1.1 Generation ................... 3-1

3.1.2 Characterization ................ 3-4

3.1.3 Storage .................... 3-7

3.1 .4 Treatment .................. 3-8

3.1.5 Waste Reduction .................. 3-9

3.1.6 Variances, Exemptions, Time Extensions . . . . . . . 3-9

3.2 PUREX AGING WASTE ..................... . . . . . 10

3.2.1 Generation .................... . 3-11

3.2.2 Characterization ................. . . . . . .

3.2.3 Storage .. . . . . . . . . . . . . . . 3-12

3.2.4 Treatment ..................... . . . . . 12

3.2.5 Waste Reduction ................... 3-13

3.2.6 Variances, Exemptions, Time Extensions ....... 3-13 
3.3 SINGLE-SHELL TANK WASTE . . . . . . . . . . . . . 3-14

3.3.1 Generation .................. 3-15

3.3.2 Characterization .. . . . . . . . . . 3-16

3.3.3 Storage .. . . . . . . . . . . . $3-18$

3.3.4 Treatment .. . . . . . . . . . . . . 3-18

3.3.5 Waste Reduction ... . . . . . . . . . . . . 3-19

3.3.6 Variances, Exemptions, Time Extensions . . . . . . 3-20

3.4 242-A EVAPORATOR PROCESS CONDENSATE . . . . . . . . . . . . 3-20

3.4.1 Generation ................... 3-21

3.4.2 Characterization ................ 3-21

3.4 .3 Storage ..................... . 3-22

3.4 .4 Treatment ................... 3-23

3.4.5 Waste Reduction ................ . . 3-24

3.4.6 Variances, Exemptions, Time Extensions . . . . . . 3-24

3.54843 SODIUM STORAGE FACILITY WASTE . . . . . . . . . . . 3-25

3.5.1 Generation .................. 3-26

3.5.2 Characterization .. . . . . . . . . . 3-26

3.5.3 Storage ... . . . . . . . . . . . . 3-27

3.5.4 Treatment ................... 3-27

3.5.5 Waste Reduction ................. . 3-28

3.5.6 Variances, Exemptions, Time Extensions . . . . . . 3-28

3.6 PUREX AMMONIA SCRUBBER WASTE . . . . . . . . . . . . 3-29

3.6.1 Generation ................... . 3-29

3.6.2 Characterization ............... 3-29

3.6.3 Storage ..................... . 3-31

3.6.4 Treatment .................... . 3-31

3.6.5 Waste Reduction ................ 3-31

3.6.6 Variances, Exemptions, Time Extensions . . . . . . 3-32

3.7 PUREX PROCESS CONDENSATE ................ . . . . . . . .

3.7.1 Generation ................... 3-32

3.7.2 Characterization ............... 3-33

3.7 .3 Storage . . . . . . . . . . . . . . 3-34

3.7.4 Treatment ................... 3-34

3.7.5 Waste Reduction .................. 3-34

3.7.6 Variances, Exemptions, Time Extensions . . . . . . 3-35

3.8 HEXONE WASTE ...................... 3-35

3.8.1 Generation ................. . 3-35

3.8.2 Characterization ................ . 3-36

3.8.3 Storage ...... . . . . . . . . . . . . 3-36

3.8.4 Treatment .................. . 3-36

3.8.5 Waste Reduction ............... 3-37

3.8.6 Variances, Exemptions, Time Extensions ...... . 3-37

3.9 183-H SOLAR EVAPORATION BASINS WASTE .......... 3-38

3.9.1 Generation .................. 3-38

3.9.2 Characterization .............. 3-39

3.9.3 Storage ... . . . . . . . . . . . . . 3-42

3.9.4 Treatment ................... 3-42

3.9.5 Waste Reduction ................... . 3-42

3.9.6 Variances, Exemptions, Time Extensions . . . . . . 3-42

3.10 PUREX STORAGE TUNNEL 2 WASTE (Mercury)........... . 3-43

3.10.1 Generation .................. 3-43 
3.10.2 Characterization ................3-44

3.10.3 Storage .................. . 3-45

3.10 .4 Treatment ... . . . . . . . . . . . 3-46

3.10.5 Waste Reduction .. . . . . . . . . . . . . . 3-46

3.10.6 Variances, Exemptions, Time Extensions . . . . . . . 3-46

3.11 PUREX STORAGE TUNNELS 1 AND 2 WASTE (LEAD) AND
STORAGE TUNNEL 2 WASTE (SILVER)

3.11.1 Generation ..... . . . . . . . . . . . . 3-47

3.11.2 Characterization ............... 3-47

3.11.3 Storage .................... . 3-48

3.11.4 Treatment ................. 3-49

3.11.5 Waste Reduction .. . . . . . . . . . . . . 3-49

3.11.6 Variances, Exemptions, Time Extensions ...... . 3-50

3.12 PUREX CONTAINMENT BUILDING (LEAD AND CADMIUM) . . . . . . . 3-50

3.12.1 Generation .................. 3-50

3.12.2 Characterization .............. 3-51

3.12.3 Storage .................... . 3-52

3.12.4 Treatment .................. . 3-52

3.12.5 Waste Reduction ................. 3-53

3.12.6 Variances, Exemptions, Time Extensions . . . . . . . 3-53

3.13 CENTRAL WASTE COMPLEX STORED LOW-LEVEL, TRANSURANIC, AND

POLYCHLORINATED BIPHENYL WASTE . . . . . . . . . . . . 3-53

3.13.1 Generation .................. . 3-55

3.13.2 Characterization ............... 3-58

3.13.3 Storage ..................... . 3-59

3.13.4 Treatment .................. 3-60

3.13.5 Waste Reduction ................. 3-64

3.13.6 Variances, Exemptions, Time Extensions . . . . . . . 3-64

3.14 RETRIEVABLY STORED LOW-LEVEL AND TRANSURANIC WASTE . . . . . . 3-65

3.14.1 Generation .................... 3-66

3.14.2 Characterization ................ 3-66

3.14 .3 Storage .................. 3-67

3.14.4 Treatment .................... . 3-70

3.14.5 Waste Reduction .................. 3-70

3.14.6 Variances, Exemptions, Time Extensions . . . . . . . 3-70

3.15 TRANSURANIC WASTE STORAGE AND ASSAY FACILITY STORED WASTE : . 3-71

3.15.1 Generation ................... 3-71

3.15.2 Characterization .............. 3-72

3.15.3 Storage .. . . . . . . . . . . . . . 3-73

3.15 .4 Treatment ................... 3-73

3.15.5 Waste Reduction .. .............. 3-74

3.15.6 Variances, Exemptions, Time Extensions . . . . . . 3-74

$3.16303-K$ STORED WASTE .................... . . 3-74

3.16.1 Generation .................... 3-75

3.16.2 Characterization .............. . 3-76

3.16.3 Storage .................... . 3-77

3.16.4 Treatment ................. . 3-77

3.16.5 Waste Reduction ................ 3-78

3.16.6 Variances, Exemptions, Time Extensions . . . . . . 3-78 
4.0 REFERENCES . . . . . 4-1

4.2 CODE OF FEDERAL REGULATIONS AND FEDERAL REGISTER . . . . 4-3

4.3 FEDERAL AND STATE ACTS . . . . . . . . . . . 4-4

4.4 WASHINGTON STATE REGULATIONS .............. 4-4 APPENDIX

A INVENTORY DATA CORRELATION ................ A-i 


\section{LIST OF FIGURES}

2-1 Operating Schedules for Units Managing Land Disposal

Restricted Wastes ................ F2-1.1

2-2 Hanford Tank Waste Remediation System . . . . . . . . F2-2.1

2-3 Plutonium-Uranium Extraction Aging Waste Treatment

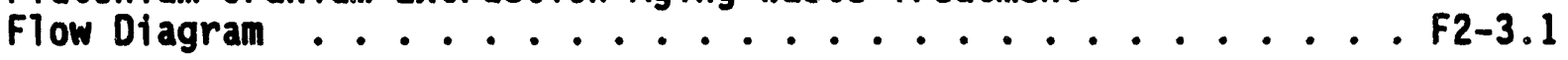

2-4 Central Waste Complex Stored Waste, Retrievably

Stored Waste, 183-H Solar Bas in Waste, and 303-K

Waste Treatment Flow Diagram .............. F2-4.1

3-1 Double-She11 Tank Space Summary . . . . . . . . . . . F3-1.1

3-2 Tank Waste Remediation System Treatment Block Diagram. . . . F F-2.1

3-3 PUREX Aging Waste Transfers to Aging Waste Storage . . . . . F3-3.1

$:-4$ Relative Proportions of Supernatant, Sludge, and Salt Cake in

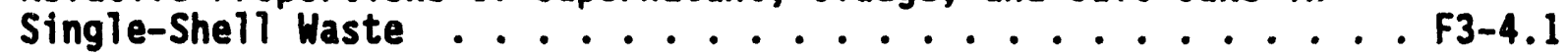

3-5 Single-Shell Tank Waste Inventory by Tank Farm . . . . . . F3-5.1

3-6 Process Flow Diagram for 242-A Evaporator . . . . . . . . F3-6.1

3-7 Historical Process Flow Diagram for PUREX Ammonia Scrubber

Waste Generation .................. F3-7.1

3-8 Ammonia Scrubber Waste Transfers to Double-Shell Tanks . . . . F3-8.1

3-9 Historical Process Flow Diagram for PUREX Process Condensate . . F3-9.1

3-10 Process Flow Diagram for Hexone Waste Processing and Disposal. F3-10.1

3-11 Plan View of Existing and Planned 200 West Area Facilities . . F3-11.1

3-12 Process Flow Diagram for Proposed Waste Receiving and

Processing Facility Module 2 ............ F3-12.1

3-13 Waste Receiving and Processing Modules 1, 2A, and 2B .... F3-13.1

3-14 Typical Configuration of Retrievable Storage Unit for

Contact-Handled Waste .............. F3-14.1

3-15 Typical Configuration of a Retrievable Storage Unit for

Remote-Handled Waste ............... F3-15.1

3-16 Transuranic Storage and Assay Facility Floor Plan . . . . . F3-16.1 


\section{LIST OF TABLES}

1-1 Stream Names for the Hanford Land Disposal Restrictions PIan for Mixed Wastes .................. . T1-1.1

2-1 Summary of Annual Waste Generation Projections ........ T2-1.1

2-2 Waste Stream Characterization . . . . . . . . . . T2-1.1

2-3 Hazardous Waste vesignations .............. T2-3.1

2-4 Storage Unit Characteristics . . . . . . . . . . T2-4.1

2-5 Stored Waste Characteristics ............ T2-5.1

2-6 Treatment of Land Disposal Restricted Waste for Disposal . . . T2-6.1

2-7 Waste Reduction Activities for Hanford Site Land Disposal Mixed Wastes............... T2-7.1

3-1 Plutonium Finishing Plant Radioactive Liquid Waste Stream Composition ................ T3-1.1

3-2 Chemical Concentrations in Double-Shell Tank Waste . . . . T3-2.1

3-3 Waste Generation for Various Facilities and Programs

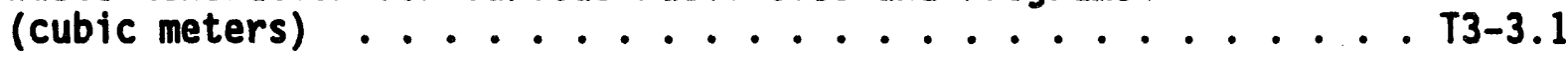

3-4 Plutonium-Uranium Extraction Aging Waste Composition ..... . T3-4.1

3-5 Sample Analysis for Plutonium-Uranium Extraction Aging Waste Stored in Tanks 241-AZ-101 and 241-AZ-102 .......... T3-5.1

3-6 Estimated Mass of Nonradioactive Chemical Components of Existing Single-Shell Tank Waste after Completion of Jet Pumping . . . . T3-6.1

3-7 Hanford Sice Single-Shell Tank Releases . . . . . . . . . . T3-7.1

3-8 Analytes Detected in 242-A Evaporator Process Condensate . . . T3-8.1

3-9 Analytes Reported in Plutonium-Uranium Extraction Plant Ammonia Scrubber Discharge ............... T3-9.1

3-10 Analyses for Plutonium-Uranium Extraction Plant Ammonia Scrubber Feed Stored in Double-Shell Tanks ........ T3-10.1

3-11 Analytes Reported in the Plutonium-Uranium Extraction Plant Process Condensate ............... T3-11.1

3-12 Analyses of Hexone Waste. . . . . . . . . . . T3-12.1

3-13 Routine Wastes Discharged to 183-H Solar Evaporator Basins . . T3-13.1 
DOE/RL-94-21

TABLES (Cont.)

3-14 Plutonium-Uranium Extraction Plant Storage Inventories . . . T3-14.1

3-15 Projected Generation of Central Waste Complex Stored Low-Level and Transuranic Waste . . . . . . . . . . . T3-15.1

3-16 Designation of 303-K Facility Stored Waste (December 31, 1993) T3-16.1 
DOE/RL-94-21

This page intentionally left blank. 
because both low-level and high-level tank wastes will now be subjected to vitrification at temperatures high enough to extract or destroy regulated organics to levels below treatment standards in the final treated waste form.

\subsection{ASSUMPTIONS}

This section lists key milestones and assumptions used to prepare this plan.

The most significant Tri-Party Agreement (Ecolooy et a1. 1992) milestones related to the management of LDR waste are identified below, including anproved change requests.

- Complete separation of tank waste into low-activity and high-activity fractions by December 2028 (M-50-00). This mile:tone includes initiation of operations by December $2004(M-50-02)$ to support operation of LLW treatment facility.

- Complete vitrification of LLW by December 2028 (M-60-00). The waste treatment facility (vitrification) will begin operations in June 2005 (M-60-05). This facility replaces the former LLW disposal method (grout), which has been canceled because of negative public opinion.

- Complete vitrification of high-level waste (HLW) by December 2028 $(M-51-00)$. Operation of the HLW Vitrification Plant will be delayed until December 2009 (M-51-03).

- Construct two new DSTs by December 1997 and up to four additional DSTs by December 1998 (M-42-00).

- Complete SST interim stabilization by September 2000 (M-41-00).

- Complete closure of all SST farms by September 2004 (M-45-00). This milestone includes a requirement to initiate tank waste retrieval from one SST by December 2003 (M-45-05T1).

- Issue Tank Characterization Reports for all SSTs and DSTs by September 1999 (M-44-00).

- Complete construction and initiate operations of expanded laboratory hot cells for high-level mixed waste by June 1994 (M-11-00).

- An initial commitment was to complete construction and initiate operation of a low-level mixed waste laboratory by January 1992 (M-14-00). U.S. Department of Energy, Richland Operations Office (RL) has decided to use offsite laboratory support for low-level mixed waste analysis and thereby did not meet this milestone. As a participant in the dispute resolution process on Milestone M-14-00, $\mathrm{RL}$ agreed to a $\$ 100,000$ penalty. It was also determined that it must be demonstrated that analysis needs can be satisfied using a combination of commercial offsite laboratory capacity and a downsized onsite laboratory that has been built. The new M-14-00 
requires compliance with the senior executive committee agreement on resolution of the M-14-00 change request dispute by October 1995 . Milestone M-14-03 specifies that the Waste Sampling and Characterization Facility will initiate operations in Ipril 1994. Milestone M-14-01 requires commencement of local commercial laboratory operations in October 1995.

- Initiate operation of 242-A Evaporator Effluent Treatment Facility (ETF) by June $1995(M-17-14)$.

- Issue tank characterization reports for 177 HLW tanks by September $1999(M-44-00)$.

- Complete Waste Receiving and Processing (WRAP) Facility, Module 1, construction and initiate operations by March 1997 (M-18-00).

- Complete WRAP Facility, Module $2 A$, construction and initiate operations by September 1999 (M-19-00).

The following are key assumptions that have been used to develop the treatment plans and schedules for DST waste (WHC 1990a) and assumptions related to the use of tank space.

- The pretreatment methods to be developed will include acceptable technology to separate the waste into LLW and HLW so that the bulk of chemical waste is in the LLW, and the bulk of radionuclides are in the HLW.

- Pretreated waste from al1 DSTs and SSTs will be provided to the LLW and HLW vitrification facilities, using selective blending if necessary.

- The level of organics in DST and SST waste received from pretreatment will be treatable by vitrification, and the glass waste forms will fully comply with leachability requirements.

- Space in DSTs, including six proposed new tanks, will be available to support DST and SST waste disposal activities.

- The 242-A Evaporator will restart in March 1994. A treatment unit for 242-A Evaporator process condensate will be available in June 1995.

- The PIutonium-Uranium Extraction (PUREX) Plant was notified to begin shutdown activities in September 1992. Stored irradiated reactor fuel will not be processed in the PUREX Plant; thus, no additional DST waste will be generated.

- During PUREX Plant shutdown, no new PUREX aging waste, PUREX process condensate, or PUREX ammonia scrubber waste will be generated. Any future PUREX process condensate and PUREX ammonia scrubber 
condensate streams will not be dangerous wastes. As part of the cleanup activitios in PUREX and B Plant, waste may be sent to aging waste tank storage. (In CY 1993, 27,000 gallons were transferred to aging waste DSTs.)

- Liquid waste from SSTs will continue to be transferred to DSTs as part of the stabilization program for the SSTs.

- The HLW and LLW vitrification processes will recycle all liquid effluent streams (i.e., they will not produce waste streams subject to the LDRs that require tank storage).

\subsection{SCHEDULE AND MECHANICS OF PLAN UPDATE}

Information in the baseline plan will be updated by additional future annual reports in accordance with Tri-Party Agreement (Ecology et a1. 1992) Milestone M-26-01. The annual reports include the following:

- Addition of new LDR waste streams as they are identified or regrouped

- Revision of the stream generation rates to reflect current operating plans and schedules

- Revision to treatment plans and schedules to reflect further defined waste treatments and treatment schedules

- Revision to the stream characterizations to reflect additional sample analyses or process changes

- Revision to the compliance status of the units to reflect future compliance assessments and permitting activities

- Reevaluation of the adequacy of the capacity of current units for the storage of LDR waste

- Addition of new or proposed milestones, as applicable.

\subsection{Milestone PLANNING PROCESS}

Milestones and work schedules for activities related to the management of LDR mixed waste will be consistent with the work schedules contained in Appendix D of the Tri-Party Agreement (Ecology et al. 1992) and the annual update to the work schedule. The scope of these schedules includes interim milestones and additional target dates to accomplish the major milestones contained in Section 2.0 of the Tri-Party Agreement. Summary milestone schedules for activities related to the management of LDR mixed waste are discussed in Chapter 3.0 of the Tri-Party Agreement. Any new or additional LDR milestones, as well as changes to approved LDR milestone schedules, will be implemented via the Change Control system process defined in Section 12.0 of the Tri-Party Agreement. 
Tri-Party Agreement Milestone M-26-01 (Ecology et al. 1992) also requires that appropriate new milestones be proposed through this annual report. No new milestones are proposed for this reporting period. The recent Tri-Party Agreement renegotiation added a significant number of milestones (Amendment 4 approved January 25, 1994), including many regarding SST and DST retrieval and treatment.

The LDR milestone planning process exercised by DOE and its contractors also involves consideration of DOE and federal budget process, integration with other concurrent Hanford Site operations (including waste management and environmental restoration activities), and overall sitewide regulatory compliance and coordination with other milestone initiatives described in the Tri-Party Agreement. Because these planning elements are numerous and complex, coordination and resolution of issues will be accomplished through the ongoing project managers' and unit managers' meetings within the broader framework provided by Section $\mathbf{8 . 0}$ of the Tri-Party Agreement. Also, LDR waste management activities will be included, as appropriate, in Tri-Party Agreement quarterly progress reports, and summarized each year, as required by Milestone M-26-01.

\subsection{ACTIVITIES AND ACHIEVEMENTS}

This section summarizes major activities and accomplishments related to compliance with LDRs from about Apri1 1, 1993, through March 31, 1994.

- The renegotiations to the Tri-Party Agreement were signed into action on January 25, 1994, substantially changing the projected future of the Hanford Site cleanup.

- Successfully tested a Rotary Mode Core Sampling Drill on simulated waste.

- Completed research, development, and installation of the Hard Salt Cake Sampler and the Hydrostatic Balance System per Tri-Party Agreement Milestone M-10-13-T2.

- Continued construction of the laboratory hot cell complex, which is scheduled to be operational in June 1994 for analysis of high-level radioactive mixed waste.

- Submitted selection criteria, retrieval approach, and recommendation for Tank 106-C as the first SST to be retrieved.

- Issued the Pretreatment Technology Plan, defining the long-range integrated program for Tank Waste Remediation System pretreatment development, and completed the Waste Pretreatment Feed Optimization study.

- Obtained 20 core samples and issued safety screening analyses for 14 SSTs.

- Obtained 10 auger samples and issued safety screening analyses results for 7 samples. 
- Completed characterization of three DST tanks (102-AP, 105-AP, and 106-AN).

- Issued the approved Multifunction Waste Tank Facility advanced Conceptual Design Report.

- Initiated settling test for neutralized current acid waste (NCAW) in-tank solids washing, completing Milestone M-02-00-T07.

- In the process of completing the paperwork and obtaining shipping containers to remove all waste from the 4843 Sodium Storage Facility. Per current planning, waste will be moved in summer 1994 to the Solid Waste Operations Complex.

- Several activities related to the Federal Facilities Compliance Act (FFCA) of 1992 were completed in 1993. The Mixed Waste Inventory Report for Hanford Site mixed wastes was submitted to U.S. Department of Energy-Headquarters (DOE-HQ). The chief financial officer's report questionnaire was completed and forwarded to DOE-HQ, and Conceptual Site Treatment Plan Tables 8-1 and 8-2 were submitted as requested. Also, a data call for all mixed waste inventory data was completed and submitted to compile a national database for submittal to the states. It will also determine a national treatment strategy for all DOE sites.

- National Treatability groupings established for the FFCA by DOE-HQ have been adopted at the Hanford Site and have been used in all submittals for the FFCA responses.

- Initiated construction of Project W-025, Mixed Waste Disposal Trench. This is a geotextile-lined trench with a leachate collection system that meets LDR requirements and that will dispose of RCRA-compliant low-level mixed waste. The design capacity of the trench is $23,000 \mathrm{~m}^{3}$ of packaged waste. It is located in the 200 West Area and will be completed in spring 1994.

- Initiated the study of privatization of various Hanford Site solid waste operations. Mixed waste treatment, storage, and disposal; incineration; and burial ground operations have been considered as viable options for privatization. Discussions continue with interested parties, DOE-RL, and the state.

- A backlog of dangerous waste had accumulated in some generating units in excess of the 90-day regulatory limit. An internal assessment completed in June 1992 identified container management problems. A subset of the backlog waste ("unknowns") is at T Plant for opening, sampling, and repackaging. Approximately 201 drums have been processed and are being shipped to the Central Waste Complex (CWC). Repackaging of 58 boxes was completed in 1994. The Waste Analysis P1an for the backlog waste program was negotiated with and approved by Ecology. More than 5,000 containers were managed through this program. Further details are located in Section 3.1.3.1 of this report. 
- A moratorium on offsite shipment of potentially radioactive hazardous and polychlorinated biphenyl (PCB) wastes was placed by DOE-HQ. RCRA LDR wastes that are believed to not be radioactive remain in storage onsite. This is inconsistent with the prohibition on such storage. On September 30, 1993, DOE-HQ approved proposed radioactive determination procedures and conditionally lifted the moratorium. Efforts have begun to sample wastes stored at 616 and CWC for radioactive content. Nonradioactive waste will be released for offsite disposal.

- Six additional submarine reactor compartments were received for storage in the 200 West Area, bringing the total to 36.

- Received approval to initiate construction of WRAP Module 1 (KD3 authorization) project $W-026$, Tri-Party Agreement Milestone M-18-00, from DOE-HQ on November 4, 1993. Groundbreaking for the construction of WRAP will take place in April 1994.

- Transferred a portion of waste in SSTs BX-110 and BX-111 to DSTs to reduce leakage.

- The spent distillation vessels from hexone waste distillation were overpacked and moved to mixed waste storage. Nine hundred gallons of solvent-saturated water are scheduled to be incinerated offsite in 1994. (The much larger quantities of distilled hexone were incinerated offsite in previous years.)

- The DOE-HQ in December 1993 announced its decision to begin the process of shutting down the Fast Flux Test Facility (FFTF). The facility had been in a hot standby condition since April 1993. 


\subsection{INTRODUCTION}

The baseline land disposal restrictions (LDR) plan was prepared in 1990 in accordance with the Hanford Federal Facility Agreement and Consent Order (commonly referred to as the Tri-Party Agreement) Milestone M-26-00 (Ecology et a1. 1992). The text of this milestone is telow.

LDR requirements include limitations on storage of specified hazardous wastes (including mixed wastes). In accordance with approved plans and schedules, the U.S. Department of Energy (DOE) shall develop and implement technologies necessary to achieve full compliance with LDR requirements for mixed wastes at the Hanford Site. LDR plans and schedules shall be developed with consideration at other action plan milestones and will not become effective until approved by the U.S. Environmental Protection Agency (EPA) (or Washington State Department of Ecology [Ecology]) upon authorization to administer LDRs pursuant to Section 3006 of the Resource Conservation and Recovery Act of 1976 (RCRA). Disposal of LDR wastes at any time is prohibited except in accordance with applicable LDR requirements for nonradioactive wastes at all times. The plan will include, but not be limited to, the following:

- Waste characterization plan

- Storage report

- Treatment report

- Treatment plan

- Waste minimization plan

- A schedule depicting the events necessary to achieve full compliance with LDR requirements

- A process for establishing interim milestones.

The original plan was published in October 1990. This is the fourth of a series of annual updates required by Tri-Party Agreement Milestone M-26-01. A Tri-Party Agreement change request approved in March 1992 changed the annual due date from October to April and consolidated this report with a similar one prepared under Milestone M-25-00. The reporting period for this report is from April 1, 1993, to March 31, 1994.

The 1990 baseline plan was a follow-on document to both the National Report on Prohibited Wastes and Treatment Options (DOE 1990) (commonly referred to as the National Report), which identified all solvent ( 40 Code of Federal Regulations [CFR] 268.30) and California List ( 40 CFR 268.32) wastes that are restricted from land disposal, and a subsequent effort by $D O E$ (WHC 1990d) to identify any additional waste that was restricted from land disposal as a result of First-, Second-, and Third-Third LDRs promulgation (55 Federal Register [FR] 22520). 


\subsection{BACKGROUND ANO PURPOSE}

On September 19, 1989, DOE entered into a federal facilitifis compliance agreement with the EPA and the Colorado Department of Health regarding the storage of certain radioactive mixed wastes at the Rocky Flats Plant. The agreement required the DOE to prepare and submit the National Report to the EPA. This report (DOE 1990) was submitted to EPA in January 1990 . It included information on a 11 DOE sites that store radioactive mixed waste subject to the LDRs in effect at the time of report preparation.

Since that time additional LDRs for dangerous waste have been promulgated by the EPA (55 FR 22520). These restrictions resulted in additional waste being restricted from land disposal. These wastes were not included in the National Report. To assess the impact of these new restrictions on DOE facilities, a survey of all DOE sites was conducted by DOE to identify any additional waste that was restricted from 1 and disposal as a result of this Thirds rule.

In 1994, revised WAC 173-303 regulations incorporated federal LDR standards by reference.

This report describes the generation and management of LDR mixed waste generated, treated, and stored at the Hanford site. Hazardous waste that is not a mixed waste is not included in this report. Discussions focus on the hazardous aspects of mixed wastes, although treatment, storage, and disposal are frequently complicated by the radioactive components. This report discusses the LDR mixed waste managed at the Hanford Site by a combination of point of gerieration and current storage locations. The waste is separated into groups based on the future treatment of the waste before disposal. This grouping resulted in the definition of 16 groups or streams of LDR waste. The 16 stream names used for this plan are shown in Table 1-1. Where a "stream" is actually a storage unit, the individual waste streams that make it up are discussed in this report as applicable.

The 16 waste streams identified for this report combine several of the waste streams identified in the National Report and the case-by-case extension petition. The National Report included solvent waste (40 CFR 268.30) and California List (40 CFR 268.32) wastes, whereas the case-by-case petition was to include all nonsolvent waste that was restricted from land disposal. This report encompasses the Hanford Site-specific aspects of the National Report (DOE 1990) and the case-by-case petition, as well as newly identified LDR waste.

Discussions with the regulators were completed in 1993 regarding major modifications to the Tri-Party Agreement milestones, particularly those that address the Tank Waste Remediation System. Included were key areas of this report, such as modifying concepts of single-shell tank (SST) and double-shell tank (DST) waste retrieval and characterization, changes to the Hanford Waste Vitrification Plant (HWVP) schedule, and replacement of the grout treatment system with a new low-level waste (LLW) vitrification facility. These changes were approved on January 25, 1994, and the new milestones are incorporated into this report. In many ways, these changes simplify LDR compliance, 
Table 1-1. Stream Names for the Hanford Land Disposal

Restrictions Plan for Mixed Wastes. (sheet 1 of 2)

\begin{tabular}{|c|c|}
\hline Stream name & Waste source \\
\hline 1. DST Waste & $\begin{array}{l}\text { Widely varying wastes from chemical } \\
\text { separations processes (e.g., PUREX } \\
\text { Plant, PFP, cesium and strontium } \\
\text { separations) used from } 1970 \text { to date }\end{array}$ \\
\hline $\begin{array}{l}\text { 2. PUREX Aging Waste (inventory in } \\
\text { DSTs) }\end{array}$ & $\begin{array}{l}\text { First extraction column fission } \\
\text { products from PUREX PIant }\end{array}$ \\
\hline 3. SST Waste (inventory)" & $\begin{array}{l}\text { Waste from spent nuclear fue } 1 \\
\text { processing between } 1944 \text { and } 1980\end{array}$ \\
\hline $\begin{array}{l}\text { 4. 242-A Evaporator Process } \\
\text { Condensate }\end{array}$ & $\begin{array}{l}\text { Condensed vapor from concentrating } \\
\text { DST waste }\end{array}$ \\
\hline $\begin{array}{l}\text { 5. } 4843 \text { Sodium Storage Facility } \\
\text { Waste (inventory) }\end{array}$ & Waste sodium from FFTF operations \\
\hline $\begin{array}{l}\text { 6. PUREX Ammonia Scrubber Waste } \\
\text { (inventory in DSTs) }\end{array}$ & $\begin{array}{l}\text { Waste generated from adsorption of } \\
\text { gaseous ammonia from fuel processing } \\
\text { operations }\end{array}$ \\
\hline $\begin{array}{l}\text { 7. PUREX Process Condensate } \\
\text { (generated during PUREX } \\
\text { operation, inventory in DST) }\end{array}$ & $\begin{array}{l}\text { Condensed vapors from PUREX Plant } \\
\text { operations }\end{array}$ \\
\hline $\begin{array}{l}\text { 8. Hexone Waste (has been treated } \\
\text { offsite) }\end{array}$ & $\begin{array}{l}\text { Hexone that had been planned for use in } \\
202-S \text { solvent extraction }\end{array}$ \\
\hline $\begin{array}{l}\text { 9. 183-H Solar Evaporation Basins } \\
\text { Waste (inventory) }\end{array}$ & $\begin{array}{l}\text { Solid remains from solar evaporation } \\
\text { basins for } 300 \text { Area fuel fabrication } \\
\text { wastes, } 1973 \text { to } 1985\end{array}$ \\
\hline $\begin{array}{l}\text { 10. PUREX Storage Tunnel } 2 \text { Waste } \\
\text { (mercury) }\end{array}$ & $\begin{array}{l}\text { Mercury sealed in discarded PUREX fuel } \\
\text { dissolvers }\end{array}$ \\
\hline $\begin{array}{l}\text { 11. PUREX Storage Tunnels } 1 \text { and } 2 \\
\text { Waste ( } 1 \text { ead) and Storage } \\
\text { Tunnel } 2 \text { Waste (silver) }\end{array}$ & $\begin{array}{l}\text { Lead from discarded equipment and } \\
\text { shielding; silver from discarded silver } \\
\text { reactors }\end{array}$ \\
\hline $\begin{array}{l}\text { 12. PUREX Containment Building } \\
\text { (lead and cadmium) }\end{array}$ & $\begin{array}{l}\text { Discarded lead and cadmium shielding } \\
\text { and weights from PUREX }\end{array}$ \\
\hline $\begin{array}{l}\text { 13. Central Waste Complex Stored } \\
\text { Low-Level, Transuranic, and PCB } \\
\text { Waste }\end{array}$ & $\begin{array}{l}\text { Onsite and offsite solid wastes from } \\
\text { many generators, primarily from routine } \\
\text { operations after 1987. }\end{array}$ \\
\hline $\begin{array}{l}\text { 14. Retrievably Stored Low-Level } \\
\text { and Transuranic Wastes } \\
\text { (inventory) }\end{array}$ & $\begin{array}{l}\text { Containers of contaminated debris } \\
\text { generated onsite and offsite up to } \\
1987 \text {. }\end{array}$ \\
\hline
\end{tabular}


Table 1-1. Stream Names for the Hanford Land Disposal Restrictions Plan for Mixed Wastes. (sheet 2 of 2)

\begin{tabular}{|l|l|}
\hline \multicolumn{1}{|c|}{ Stream name } & \multicolumn{1}{|c|}{ Waste source } \\
\hline 15. TRUSAF Stored Waste & $\begin{array}{l}\text { Transuranic waste from onsite and } \\
\text { offsite, packaged for eventual WIPP } \\
\text { disposai. }\end{array}$ \\
\hline 16. 303-K Stored Waste & $\begin{array}{l}\text { Temporary storage of 300 Area fuel } \\
\text { fabrication solid and } 1 \text { iquid wastes. }\end{array}$ \\
\hline
\end{tabular}

"No longer being generated.

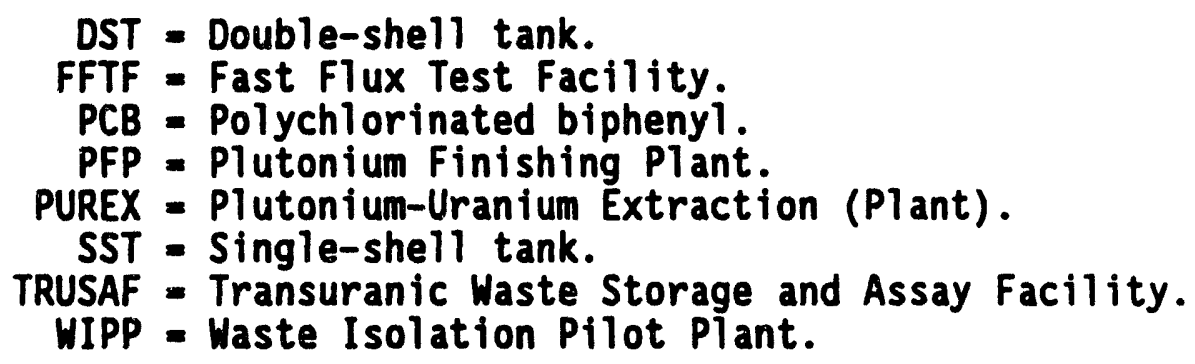




\subsection{SITE SUMMARY}

This section summarizes the generation, characterization, storage, treatment, and reduction of radioactive LDR waste at the Hanford Site. It also discusses the variances, exemptions, and time extensions required to manage this waste within the requirements established by 55 FR 22520 and 40 CFR 268.

\subsection{MASTE GENERATION}

The projected volumes of radioactive mixed waste to be generated are shown in Table 2-1. The assumptions governing these generation rates are discussed in Chapter 1.0, Section 1.2. These assumptions are summarized below.

- The operation of waste pretreatment, treatment and disposal units will proceed as scheduled in the Tri-Party Agreement (Ecology et al. 1992).

- It is assumed that obligations of DOE arising under the Tri-Party Agreement will be fully funded. The DOE will take all necessary steps to obtain timely funding to meet its obligations under the Tri-Party Agreement. Ecology and EPA will assist U.S. Department of Energy, Richland Operations Office (RL) in determining the specific tasks required to support the corresponding negotiated work schedule for each fiscal year, but will not become involved with the internal DOE budget process.

- Site production plants (e.g., PFP) will continue to operate within their current planning bases.

The annual waste generation volumes presented in Table 2-1 represent the current best estimates of future waste generation for each of the LDR mixed waste streams or storage units. These estimates are based on detailed evaluation of plant operating schedules, past operating history, and projections of future waste generation. The projected generation volumes may be higher or lower than the actual generation rates because of changes in waste treatment or production schedules or waste minimization activities.

Decommissioning and remediation activities are anticipated to generate large volumes of contaminated soils and debris (e.g., contaminated structures, drums, tanks, piping, equipment, and cleanup debris) that may be subject to regulation under the LDR Program. Volumes will be defined during the Programmatic Environmental Impact Statement (EIS) process. Volumes cannot be accurately determined until RCRA Facility Investigation/Corrective Measures Studies, Comprehensive Environmental Response, Compensation, and Liability Act of 1980 (CERCLA) Remedial Investigation/Feasibility Studies, and Decontanination and Decommissioning Work Plans have been completed and remedies have been selected. Treatment standards for debris were promulgated by EPA on August 18, 1992 (57 FR 37194). Specific treatment standards for LDR soils have not been promulgated as of March 1994. However, upon promulgation of these standards, treatment and possibly expanded storage capacity for 
generated wastes from decommissioning and remediation activities will require planning and development. Should promulgated standards not be feasible for these soils and debris, variances from such standards will be applied for. Extended storage of these wastes would be allowable pursuant to Tri-Party Agreement provisions dealing with LDR waste. Planning information as it develops for these wastes will be incorporated into future revisions of this report.

\subsection{WASTE CHARACTERIZATION}

Radioactive mixed waste at the Hanford Site has been characterized, as documented in this plan, based on current process knowledge and, where available, waste sample analyses. Sampling and characterization of waste will continue until the waste is disposed. Future characterization plans for the waste are summarized in Table 2-2. Individual waste streams are described in Chapter 3.0.

The dangerous waste designations for the waste in storage are summarized in Table 2-3. This table shows the dangerous waste codes applicable to each of the waste streams. The assigned dangerous waste codes are based on the generation process and analyses of the waste streams. The waste designations are based on the best available information. However, future waste characterization may show that additional or fewer waste codes are applicable to a waste stream. Any changes will be included in updates of this report.

The waste stored in the SSTs, the DSTs, and the silver nitrate waste stored in the PUREX Storage Tunnels have been assigned the D001 (ignitable) waste designation because of the presence of oxidizers, nitrates, and/or nitrites. They are not ignitable by themselves, and the designation results from the possibility of reaction with other materials.

Using the FOO1 through F005 (spent halogenated and nonhalogenated solvents) waste codes to characterize the waste stored in the SSTs and DSTs has been assigned to the waste not because the waste contains significant quantities of spent solvents, but because small quantities of waste discharged to the tanks in the past may have contained spent solvents. The potential for the past discharge of spent solvents to have contaminated essentially all of the waste in the tanks has resulted in the waste being designated F001 through F005. The tank waste does not comprise large quantities of organic solvents, as is typically the case for waste designated F001 through F005. The tank waste primarily is inorganic in nature with trace contamination by $\mathrm{FOOl}$ through F005 solvents.

The schedule and means for reporting waste characterization data are outlined in the Tri-Party Agreement (Ecology et al. 1992) as amended by new Section 9.6, "Data Reporting Requirements." This section states that DOE wi11 make available to Ecology and EPA all validated laboratory analytical data collected pursuant to the Tri-Party Agreement within 15 work days of data validation. Within 1 week after the laboratory data are validated, DOE will notify Ecology and EPA of its availability in the Hanford Environmental Information System. This notification will include the time and location of the sampling, the type of data available, and a list of the sample parameters 
or a target compounds list. The time limits for reporting sample analyses are SST analyses, 216 days; hot cell analyses, 176 days; and low-level and mixed waste, 126 days (after the date of sampling).

Before any sampling or analysis, the appropriate level of quality assurance/quality control will be defined and documented in accordance with Guidelines and Specifications for Preparing Quality Assurance Program Plans (EPA 1983a) and Interim Guidance and Specifications for Preparing Quality Assurance Project Plans (EPA 1983b). All laboratories that analyze samples for DOE also are required to have a quality assurance/quality control plan approved by EPA and Ecology before being used to conduct analyses.

\subsection{WASTE STORAGE}

The Hanford Site has 16 units, as defined by this report, that currently store mixed waste. These 16 units can be divided into two groups: (1) eight that are no longer actively receiving waste (SST waste, PUREX aging waste, PUREX ammonia scrubber waste, PUREX process condensate, 4843 Sodium Storage Facility Waste, hexone waste, 183-H Solar Evaporation Basins waste, and retrievably stored LLW and TRU waste); and (2) eight that are currently receiving or could receive waste for storage to await treatment and disposal (DST waste, Liquid Effluent Retention Facility [for 242-A Evaporator Process Condensate], the three PUREX tunnel streams, CWC, Transuranic Waste Storage and Assay Facility [TRUSAF], and the 303-K Facility). The key characteristics of these units are summarized in Table 2-4.

The storage unit capacity for radioactive mixed waste at the Hanford Site is projected to be adequate for all currently generated mixed waste uni il at least 1996, assuming the availability of additional storage facilities such as part of the Central Waste Complex (CWC) and up to six new DSTs. After approximately three campaigns, the LERF will be near its storage capacity for 242-A Evaporator process condensate (Chapter 3.0, Section 3.4). Current plans are to suspend 242-A Evaporator operations temporarily until waste treatment at the Effluent Treatment Facility can treat the stored process condensate and receive process condensate directly from the 242-A Evaporator. After the Effluent Treatment Facility (ETF) has treated the process condensate stored in LERF (projected to be completed in 1994), it will treat process condensate directly without further storage at the LERF.

In 1994, the currently available DSTs are essentially filled to capacity. The latest plans are to design and construct up to six additional tanks. This is in accordance with Tri-Party Agreement (Ecology et al. 1992) Milestone M-42-00, with a completion date of December 1998.

The CWC is projected to reach its capacity in 1999. This projection is based on the individual projections of all generators who ship waste to the CWC and the availability of planned storage and treatment facilities. The projection of waste generation rates is refined annually. Should future projections indicate that increased storage capacity is required, additional storage units will be constructed and permitted on an as-needed basis. 
The storage units for mixed waste at the Hanford Site have not released any dangerous constituents to the environment with the exception of the SSTs and the 183-H Solar Evaporation Basins. This has been determined through all available information such as monitoring data, inspections, and operational history. The SSTs have released an estimated 2,600 cubic meters of 1iquid waste to the ground. This estimate excludes any cooling water added to tanks after they were known to be leaking. This volume estimate currently is being reviewed for accuracy and may be revised. To minimize further releases from this storage unit, the pumpable liquid portion of the waste stored in the SSTs is being transferred to the DSTs. The amount of hazardous constituents raleased from the 183-H Solar Basins has not been estimated as this time. This will be a part of the data evaluation report for this unit.

The Part B Permit application submittal date for the mixed waste storage unit is shown in Table 2-4. The date when each unit will be in full compliance with the interim status requirements is shown as the "Compliance Date." The schedule for the permitting of the storage and treatment units currently used for mixed waste storage or planned for use in treating LDR waste is shown in Figure 2-1.

The general characteristics of the radioactive mixed waste currently in storage at the Hanford Site are summarized in Table 2-5. The table shows that as of December 31, 1993, the Hanford Site stores approximately 243,830 cubic meters of radioactive mixed waste. The bulk of this waste (97\%) is stored in the SSTs (57.2\%) and DSTs (39.8\%). The table also indicates how much waste is LLH, TRU waste, or HLW.

\subsection{WASTE TREATMENT}

The LDRs for dangerous waste (55 FR 22520 and Washington Administrative Code [WAC] 173-303-140) (WAC 1990) specify a series of treatment technologies or treatment standards for each dangerous waste code that is restricted from land disposal. If a treatment technology is specified for a waste code, that technology must treat the waste before land disposal of the treatment

residues. If a constituent concentration treatment standard is specified for a waste code, any treatment method may be used before land disposal so long as the treatment standard is met and the waste is not impermissibiy diluted. If a waste exhibits multiple waste codes, it must be treated in accordance with the technologies or constituent concentration standards associated with each of the waste codes present. Additionally, ignitable (D001) and corrosive (D002) wastes must be treated to remove any underlying hazards, in accordance with recently imposed requirements for wastes (58 FR 29860, "Land Disposal Restriction for Ignitable and Corrosive Characteristic Wastes Whose Treatment Standards Were Vacated"). For example, if a waste is ignitable (D001) and 
TCLP toxic for chromium (D007), the waste must be treated for the characteristics of ignitability, TCLP toxicity, and for the presence of any contaminants listed in the F039 list reasonably expected to be present.

This plan summarizes the treatment standards applicable and those proposed for the Hanford Site waste; included are discussions of the following waste categories:

- DST Waste (Chapter 3.0, Section 3.1)

- PUREX Agir.g Waste (Chapter 3.0, Section 3.2)

- SST Waste (Chapter 3.0, Section 3.3)

- 242-A Evaporator Process Condensate (Chapter 3.0, Section 3.4)

- 4843 Sodium Storage Facility Waste (Chapter 3.0, Section 3.5)

- PUREX Ammonia Scrubber Waste (Chapter 3.0, Section 3.6)

- PUREX Process Condensate (Chapter 3.0, Section 3.7)

- Hexone Waste (Chapter 3.0, Section 3.8)

- 183-H Solar Evaporation Basins Waste (Chapter 3.0, Section 3.9)

- PUREX Storage Tunnel 2 Waste (mercury) (Chapter 3.0, Section 3.10)

- PUREX Storage Tunnels 1 and 2 Waste (lead) and Tunnel 2 (silver) (Chapter 3.0, Section 3.11)

- PUREX Containment Building Storage (lead and cadmium) (Chapter 3.0, Section 3.12)

- CWC Stored LLW, TRU Waste, and PCB Waste (Chapter 3.0, Section 3.13)

- Retrievably Stored LLW and TRU Waste (Chapter 3.0, Section 3.14)

- Transuranic Waste Storage and Assay Facility (TRUSAF) Stored Waste (Chapter 3.0, Section 3.15)

- 303-K Stored Waste (Chapter 3.0, Section 3.16).

The applicable treatment standards (required by 55 FR 22520 and WAC 173-303-140) and the proposed treatments for the Hanford Site mixed waste are summarized in Table 2-6. All of the contributing streams to the DST system are combined as one, because all will be pretreated into HLW and LLW

${ }^{1}$ Wastes that exceed TCLP standards are not subject to the LDRs because of this characteristic. Wastes that exceed Extraction Procedure (the older test method) standards are subject to the restrictions. However, EPA prefers that facilities use the TCLP test method to determine if the waste meets treatment standards. 
streams and vitrified similarly (Table 2-6). The schedule for the operation of the treatment units is provided in Figure 2-1.

Applicable treatment alternatives are described in Chapter 3.0. The use of offsite commercial treatment technologies is currently under consideration for some waste streams. (The hexone waste stream, containing very low levels of radioactivity, has previously been incinerated off site.) The use of onsite commercial technologies is also possible. A private firm has proposed to build and operate a high-level vitrification system using French technology. This proposal is under consideration. The use of commercial technologies is likely to play a major role in site remediation work (primarily under CERCLA regulations). Certain solid waste treatment operations, such as WRAP Module IIA, are planned to be privatized.

The Tri-Party Agreement specifies the required dates for construction, startup, and waste treatment in the major treatment facilities. There are no requirements for accelerated treatment beyond these dates. All of these wastes are considered to be stored in a relatively environmentally sound manner with the exception of SST wastes and several DSTs with unique safety problems because of chemical content. Further details on accelerated treatment are located in the individual waste stream treatment discussions in Chapter 3.0.

\subsubsection{Double-Shell Tank Waste}

The DST waste consists of LLW, TRU waste, and HLW. In the interim storage mode, however, the waste is managed as $H L W$ and is evaporated at the 242-A Evaporator to reduce the tank waste volume. Before treatment for disposal, the waste will be separated (i.e., pretreated) into two streams: a LLW stream and a HLW/TRU stream. The HLW stream may undergo additional treatment as necessary to further reduce its volume and increase radionuclide loading.

Before disposal, appropriate testing of the LLW and HLW/TRU products will be conducted to ensure the waste will comply with the LDR standards. The HLW subsequently will be disposed of at a HLW repository in a still-to-be determined national location; the TRU waste may be disposed of at WIPP near Carlsbad, New Mexico; and the LLW will be disposed of near surface on the Hanford Site. Figure 2-2 depicts the DST separation, treatment, and disposal processes.

Several Hanford Site plants are planned to perform treatment and disposal processes. A pretreatment facility will be constructed to perform the necessary waste separations, with startup scheduled for December 2004 for the LLW stream and June 2008 for the HLW/TRU waste stream. Startup is scheduled for the LLW vitrification facility in June 2005 and the HLW/TRU waste vitrification facility in 2009; subsequent disposal of treated HLW will begin when a national repository is available. The schedule for these treatment processes is shown in Figure 2-1. 
DOE/RL-94-21

\subsubsection{PUREX Plant Aging Wasto}

Treatment of the PUREX Plant aging waste stored in DSTs is addressed in Section 2.4.1.

\subsubsection{Single-Shell Tank Waste}

The SST waste consists of LLW, TRU Waste, and HLW; however, in the interim storage mode it is managed as HLW. The physical forms of SST waste are sludge, salt cake, and liquid. Llquid waste, which includes supernatant and interstitial liquid within the salt cake, will be transferred to DSTs for subsequent treatment. The planning base for SSTs is to retrieve all the waste and transfer it to DSTs where it will then be separated into LLW and HLW/TRU waste fractions (via pretreatment) which will both be vitrified for disposal; the same as for DST waste shown in Figure 2-2.

\subsubsection{2-A Evaporator Process Condensate}

The 242-A Evaporator process condensate waste (containing trace organic solvents) will be stored in a surface impoundment (LERF) for a short time while the Effluent Treatment Facility (ETF) is constructed. The ETF is being designed and will be constructed and operated to destroy organic constituents and to remove radioactive and certain inorganic constituents. The ETF will treat the waste streams to aliow discharge to the ground. A petition was submitted to delist the process condensate after it is treated.

There is a Research, Development, and Demonstration permit in work that is intended to permit the testing of the proposed treatment technologies on actual waste from the 242-A Evaporator when it becomes avallable. This permit is currently in preparation by EPA, Region 10, and was originally scheduled to be issued in October 1992. The permit will allow testing of actual

242-A Evaporator process condensate at the 1706-KE Facility in the 100 Area at the Hanford Site.

\subsubsection{Sodium Storage Facility Waste}

The 4843 Sodium Storage Facility presently is not receiving additional materia1. A closure plan has been developed and transmitted to Ecology. All but one container of nonradioactive waste has been shipped off site for ultimate disposal by independent contractors. Mixed waste will be transported to the CWC. This is currently planned for summer 1994. A considered treatment for 4843 Sodium Storage Facility waste is deactivation by reacting it to form a sodium hydroxide/water solution and then reacting this solution with carbon dioxide to form sodium carbonate.

\subsubsection{PUREX Plant Ammonia Scrubber Waste}

The PUREX ammonia scrubber waste is generated when ammonia gas from the $N$ Reactor fuel decladding process is sprayed with water. In the past the ammonia-bearing solutions were bolled in a concentrator to separate the bulk 
of the entrained fission products from the ammonia scrubber discharge that was disposed in a crib. The remaining ammonia scrubber waste was transferred to DSTs.

In late 1987, it was determined that the ammonium hydroxide concentrations in the ammonia scrubber discharge sometimes exceeded $1 \%$, making it a dangerous (toxic) waste as designated by state regulations and, therefore, not appropriate for discharge to the crib. The remaining ammonia scrubber feed was no longer concentrated for discharge, but treated for tank storage and transferred as ammonia scrubber waste to underground storage tanks. The last ammonia scrubber waste was generated during December 1989. The treatment consisted of adding caustic to adjust the $\mathrm{pH}$ to greater than 12 and adding sodium nitrite to minimize tank corrosivity.

The PUREX Plant has received official notification to begin shutdown activities. Ammonia scrubber waste will no longer be generated.

\subsubsection{PUREX Plant Process Condensate}

The PUREX Plant process condensate is generated by condensing the vapors resulting from evaporative concentrations of $N$ Reactor fuel solutions.

Until 1987 the PUREX Plant process condensate stream was discharged directly to a crib if radioactivity was sufficiently low. After closure of the old crib and to prevent corrosive (pH less than 2) waste from being discharged into the new crib, potassium hydroxide was. added and the stream was routed through a tank with calcium carbonate (1 imestone) before being discharged. In early 1989 the stream was rerouted temporarily to underground storage tanks pending resolution of its dangerous waste designation. The last PUREX Plant process condensate was generated in March 1990.

The PUREX Plant has received official notification to begin shutdown activities. Process condensate will no longer be generated.

\subsubsection{Hexone Waste}

Hexone waste was removed from the storage tanks in the 200 West Area in 1990 and distilled to remove radionuclides (except for tritium). The distillate was temporarily stored in tank cars and was then trucked off site for incineration. The treatment reduced the hexone to carbon dioxide and water. Incineration is $97 \%$ complete, with the final waste batch to be incinerated in March or April 1994. Spent distillation vessels were sent to the Mixed Waste Storage complex for storage and treatment. Approximately 500 gallons of distillation tars remain in the storage tanks. A closure plan has been submitted to Ecology, and the tanks are awaiting closure.

\subsubsection{3-H Solar Evaporation Basins Waste}

The 183-H Solar Evaporation Basins waste, designated for toxicity (chromium), and trace listed commercial chemical products (formic acid, cyanide salts, vanadium pentoxide) resulted from closure of the 183-H Basins 
storage unit. The contaminants and residues remaining in the 183-H Basins were placed in containers and transported to the CWC for storage.

Subsequentiy, the waste will be treated at the WRAP Facility and disposed of in a near-surface disposal unit. The required treatment technology for formic acid is incineration; therefore, a treatability variance may be required before ultimate disposal of this waste. (The total amount of formic acid was 2 pounds diluted in 2.5 million gallons total waste volume.)

\subsubsection{PUREX Storage Tunnels 1 and 2 Waste (Lead, Morcury, and silver) and PUREX Containment Butlding (Lead and Cadmiun)}

The PUREX Plant waste includes lead solids, mercury, and stlver waste stored in the PUREX tunnels and lead and cadmium solid waste stored in the PUREX Containment Bullding. The required treatment for lead solids is microencapsulation and/or surface decontamination. If surface decontamination is selected, the treatment residue must meet the lead characteristic treatment standard of 5 milligrams per 1iter. Amalgamation or retorting and recovery are the required treatments for mercury waste. Any treatment that achieves the constituent concentration 1 imits is applicable for the silver waste. Treatments have not been selected yet.

\subsubsection{Central Haste Complex Stored Low-Level, Transuranic,} and Polychlorinatod BIphenyl Waste; TRUSAF Stored Waste; and Retrievably Stored Low-Level, Transuranic, and Polychlorinated Biphenyl Waste

Waste stored in the CWC consists of low-level and TRU mixed waste, some of which is co-contaminated with PCBs. The retrievably stored suspect-TRU waste will be assayed and separated at the WRAP Facility into TRU and low-level streams. This TRU waste plus TRU waste stored at the TRUSAF and the CWC will be certified and shipped to WIPP for disposal. The LLW will be disposed of in a near-surface disposal unit. Mixed waste will be treated as necessary to separate the radioactive and hazardous components. Retrievably stored LLW and TRU wastes are primarily contained in 0.21 -cubic-meter drums, metal boxes, wood boxes, and fiberglass reinforced plastic boxes. They are stored in various configurations of underground storage units. After retrieval, the waste is processed/treated at the WRAP Facility so it is acceptable for permanent disposal. The proposed treatments comply with the 55 FR 22520 and WAC 173-303-140 treatment requirements. The specific processes to be used currently are being selected. Also, privatization options are currentiy under study to determine feasibility and practicability. The PCBs will be stored until treatment capacity is identified. Figure 2-4 depicts the CWC treatment and disposal processes.

\subsubsection{3-K Stored Waste}

The 303-K waste consists of container-stored waste. Current plans are to move the pyrophoric chips and fines to the 304 Building for concretion and then burial as LLW, and to move the remainder of the waste to storage at the CWC for treatment by the WRAP Facility. The CWC treatment plans are discussed in Section 2.4.11. 
DOE/RL-94-21

\subsection{WASTE MINIMIZATION}

\subsubsection{Waste Minimization Program Elements}

Six basic elements comprise the overall waste minimization program: top management support, characterization of waste generated and the process that generates it, waste minimization assessments, cost allocation, technology transfer, and program evaluation.

2.5.1.1 Statement of Management Support/Comitment. The RL Manager and contractor management are committed to minimizing the generation of waste by giving preference to source reduction, material substitution, and environmentally sound recycling over treatment, storage and disposal of such waste. Management takes appropriate action to provide adequate personne1, budget, training, and resources on a continuing basts to ensure that the objectives of the waste minimization program are met.

Annual goals have been established by both RL and contractor management for all types of waste generated at the Hanford Site. Through the performance of waste minimization assessments and selection of economicaliy practicable options, the site goals are translated into specific goals for each facility.

Management support is further evidenced by including waste minimization training in the Hanford General Employee Training program, through incentives programs that reward individual and group contributions, and inclusion of waste minimization in job performance evaluations of persons having waste minimization responsibilities.

2.5.1.2 Characterization of Waste Generation. Waste that is generated is characterized to obtain information on quantity generated, hazardous constituents, and their concentration.

2.5.1.3 Periodic Maste Minimization Assessments. Waste minimization is to be considered as an integral part in the design of any new facility or the modification of an existing facility or process. Waste that is nonetheless generated will periodically be assessed for waste minimization potential through a methodology called pollution prevention opportunity assessments. This methodology requires that a pollution prevention opportunity assessment team be formed to evaluate each waste generating process selected.

The key elements for conducting pollution prevention opportunity assessments are to--

- Select a team leader who is familiar with the facility and its processes, waste generation, and waste management operations to head the team.

- Assemble a multidisciplined team and train them in the pollution prevention opportunity assessment process.

- Collect baseline information including process flow diagrams, material balances, waste quantities, and process description. 
DOE/RL-94-21

- Develop waste minimization options including costs and savings.

- Evaluate options and recommend course of action.

- Document all work on pollution prevention opportunity assessment forms and place them in the appropriate appendix of the facility's waste minimization plan.

2.5.1.4 Cost Allocation System. A cost accounting system that accounts for the "true cost" of waste that is generated by the factlity must include shortand long-term costs arising from (1) underutilization of raw materials found in the waste stream, (2) management of the wastes that are generated,

(3) waste disposal, and (4) third-party 11 abllities if the waste is improperly disposed. Associated costs will include personnel, recordkeeping, transportation, pollution control, equipment, treatment, storage, disposal, liabilits, compliance, and oversight costs.

Present1y, only estimates of some of the cost contributors are available. These estimates are combined with "known" costs to evaluate waste minimization alternatives in the process waste assessment process.

2.5.1.5 Technology Transfor. The transfer of federally developed technology between laboratories and potential users is a contractual responsibility of DOE faclitities and laboratories. Activities involving technology transfer must be coordinated through the contractor's office that has been designated to represent the facility on the Federal Laboratory Consortium for Technology Transfer. The Federal Laboratory Consortium promotes technology transfer through iinks to the public and private sectors and through support services such as training and assistance in implementing partnership opportunities. Transfer of technologies specific to waste minimization may develop from information exchange systems, workshops, or topical conferences.

2.5.1.6 Progran Evaluation. Achlevements and milestones in the program will be a part of the contractor's performance evaluation and determination of award fees. The results of this evaluation by the contractor are reported by the Pollution Prevention group of the prime contractor to RL in periodic reports.

The following success criterla are avallable to aid in the demonstration of effective waste minimization efforts:

- Reduced amount of hazardous waste

- Reduced amount of all waste

- Reduced waste management costs

- Improved regulatory compliance

- Reduced health risks

- Increased production efficiency

- Reduced accident risk

- Improved public relations.

\subsubsection{Program Objectives}

The objectives of the waste minimization program are listed below. 
- Foster a philosophy to conserve resources and minimize waste and pollution while achieving Hanford Site strategic objectives.

- Promote the use of nonhazardous materials in operations to minimize the potential risks to human health and the environment.

- Reduce or eliminate the generation of waste through input substitution, process modification, improved housekeeping, and closed-loop recycling to achieve minimal adverse effects to the air, water, and land.

- Comply with federal and state regulations and DOE requirements for waste minimization, waste reduction, and pollution prevention.

- Characterize waste streams and develop a baseline of waste generation data.

- Identify and implement methods and technologies for waste minimization.

- Target policies, procedures, or practices that may be barriers to waste minimization.

- Enhance communication of waste minimization objectives, goals, and ideas.

- Promote integration and coordination of waste generators and waste managers on waste minimization matters.

- Develop specific goals and schedules for waste minimization activities.

- Create incentives for waste minimization.

- Collect and exchange waste minimization information through technology transfer, outreach, and educational networks.

- Develop mechanisms for fully disseminating current technical information to Hanford Site users.

\subsubsection{Facility-Specific Maste Minimization}

All facilities that generate waste are required to have a waste minimization program in place. The effectiveness and implementation of the programs are audited on a regular basis. The following are key components of the program.

- To the extent practical, all mixed waste is segregated and packaged separately from LLW or TRU Waste that contains no hazardous or dangerous constituents.

- The volume of mixed waste is reduced by compaction when possible. 
- To minimize the generation of mixed waste, generators actively seek nondangerous alternatives for the dangerous constituents in their processes.

- Waste is characterized and the potential for minimization is investigated.

- Minimization goals are set annually and tracked on a quarterly basis.

- If allowed by reguletion, mixed waste is treated to remove the dangerous constituents.

- Corrosive materials are neutralized (if allowed by regulation) removing their corrosive character or packaged in a manner ensuring integrity of the containment barriers.

- Waste handling, segregation, and certification will be performed following detailed procedures when the disposal criteria are promulgated.

- A Quality Assurance Program Plan and implementing procedures are required.

Table 2-7 summarizes the waste reduction (minimization and treatment) methods currently in place or planned for the 16 waste units addressed in this plan. The table also shows schedules for implementation and the projected effectiveness of the method.

Future mixed-waste generation rates are dominated by the process condensate from the 242-A Evaporator (Table 2-1). In a typical year, more than five times more process condensate is generated than all other waste streams combined. However, the planned Effiuent Treatment Facility will reduce, by greater than $99 \%$ the volume of process condensate designated as dangerous waste.

Next to the planned treatment of the 242-A Evaporator process condensate stream, the most significant waste reduction is seen for DST waste. Evaporation of the dilute waste received into the DST reduces an annual average generation of 10,000 cubic meters to approximately 2,000 cubic meters.

The waste currently stored at the CWC will be processed at the WRAP Facility (described in Chapter 3.0, Section 3.13) before disposal. This facility will minimize the amount of LDR waste by separating the dangerous constituents from the nondangerous constituents.

In a typical year, waste reduction practices at the Hanford Site will reduce the volume by well over 100,000 cubic meters. The majority of the reduction is from treatment.

In addition to specific waste reduction sections in Chapter 3.0 of this report, waste reduction at the Hanford Site is described in the Hanford Site Annual Waste Reduction Report (RL 1991b). 


\subsection{VARIANCES, EXEMPTIONS, AND TIME EXTEMSIONS}

Removal and treatment of the Hanford Site stored mixed waste to meet LDR requirements are summarized in Section 2.4 .

The national capacity variance for Third-Third Waste (55 FR 22520) provided for a 2-year national capacity variance from the LDR for Third-Third mixed waste expiring on May 8, 1992. This variance allowed continued storage of these wastes. A Federal Register notice dated May 26, 1992, stated that EPA could not grant a case-by-case extension for Third-Third mixed waste because of questions regarding the DOE's demonstration that they have entered into binding contractual commitments to construct or otherwise provide treatment capability. A final decision has yet to be made. This extension would allow DOE to store LDR mixed wastes until May 8, 1993, and this date could be extended for up to an additional year. The Tri-Party Agreement will allow continued storage of these wastes in accordance with the schedules in the Tri-Party Agreement. The Federal Facility Compliance Act of 1992 also allows DOE facilities to store their wastes for 3 years if compliance with the Act's provisions for providing waste inventory and treatment plans are met.

If additional variances, exemptions, or time extensions are required as a result of delays in the development of treatment, storage, or disposal capacity, they will be applied for in accordance with the procedures detailed in the Tri-Party Agreement (Ecology et al. 1992). No variances have been scheduled to date.

The Tri-Party Agreement provides for extension of a schedule or a deadline upon receipt of a timely request for extension and when good cause exists for the requested extension. Any request for extension shall be submitted in writing and shall specify:

- The timetable and deadline or schedule for which the extension is sought

- The length of the extension sought

- The good cause for the extension

- Any related deadline or schedule that would be affected if the extension were granted.

Good causes for an extension include the following:

- An event of force majeure as defined in Article XLVII of the Tri-Party Agreement, subject to Ecology's reservation in Paragraph 147.

- A delay caused by another party's failure to meet any requirement of the Tri-Party Agreement

- A delay caused by invocation of dispute resolution to the extent provided by Paragraph 30 (f) and Paragraph 59(I) or judicial order 
- A delay caused, or which is likely to be caused, by an extension granted to another deadline or schedule

- Any other event or series of events mutually agreed to by the parties as constituting good cause. 
DOE/RL-94-21

This page intentionally left blank. 
Table 2-1. Summary of Annual Waste Generation Projections.*

\begin{tabular}{|c|c|c|c|c|c|}
\hline \multirow{2}{*}{ Waste strean } & \multicolumn{5}{|c|}{ Projected generation $\left(m^{3}\right)$} \\
\hline & 1996 & 1995 & $19 \%$ & 1997 & 1998 \\
\hline 1. OST Waste (before evaporation) & 7,800 & 11,300 & 11,300 & 12,100 & 11,300 \\
\hline 2. PUREX Aging Haste & 0 & 0 & 0 & 0 & 0 \\
\hline 3. Sst Haste & 0 & 0 & $\mathbf{0}$ & 0 & 0 \\
\hline 4. 242-A Eveporator Procese Condeneate & 26,600 & 31,800 & 30,600 & 13,300 & 12,500 \\
\hline 5. 4843 sodiu storege Fecility Waste & 0 & $\mathbf{0}$ & $\mathbf{0}$ & 0 & $\mathbf{0}$ \\
\hline 6. PUREX Amonia Scrubbar Maste & 0 & 0 & $\mathbf{0}$ & $\mathbf{0}$ & $\mathbf{0}$ \\
\hline 7. PUNEX Proceses Condenente & $\mathbf{0}$ & $\mathbf{0}$ & $\mathbf{0}$ & 0 & $\mathbf{0}$ \\
\hline 8. Hexome Haste & 0 & 0 & $\mathbf{0}$ & $\mathbf{0}$ & $\mathbf{0}$ \\
\hline 9. 183-H Solar Eveporation Basine Waste & $\mathbf{0}$ & 0 & 0 & $\mathbf{0}$ & $\mathbf{0}$ \\
\hline 10. PUREX storage Tunnel 2 Waste (morcury) b & $\mathbf{0}$ & $\mathbf{0}$ & $\mathbf{0}$ & $\mathbf{0}$ & $\mathbf{0}$ \\
\hline $\begin{array}{l}\text { 11. PuREX storage Tumnls } 1 \text { and } 2 \text { Wast } 8 \\
\text { (lead) and Tumbl } 2 \text { Haste (silver) }\end{array}$ & 0 & 0 & $\mathbf{0}$ & 0 & $\mathbf{0}$ \\
\hline 12. PunEX Cogtainment cuilding (lead and & 0 & $\mathbf{0}$ & 0 & $\mathbf{0}$ & 0 \\
\hline 13. Cux stored Low-Leval, TRU, and PCe Haste & 4,273 & 3,502 & 3,205 & 2,877 & 2,603 \\
\hline $\begin{array}{l}\text { 14. Rotrievably Stored Low-Level and } \\
\text { Tru Uaste }\end{array}$ & 0 & 0 & 0 & 0 & $\mathbf{0}$ \\
\hline 15.. Trusaf Stored Waste & 266 & 266 & 266 & 266 & 266 \\
\hline 16. 303-K Stored Weste & 1 & 0 & 0 & 0 & 0 \\
\hline Total Projected Generstion & 36,940 & 46.868 & 45,371 & 28,543 & 26,749 \\
\hline
\end{tabular}

These seneration rates are besed on the essuptione of chepter 1.0, section 1.2.

beneration rate depends upon the need to nove failed equipenent containing mercury, lead, end/or silver. Rel isble predictions can be mads only 1 year in edvence.

Cuc = Central Uaste Complex.

DST a Double-shell tank.

PC = Polychlorinated bipheml.

PUREX = Plutoniun-Urenium Extrection (Plent).

SST = single-shell tenk.

TRU = Transurenic.

TRUSAF = Irensurenic Waste Storege and Assay Facility. 
Table 2-2. Waste Stream Characterization. (sheet 1 of 3 )

\begin{tabular}{|c|c|c|}
\hline Waste stream & Schedule & $\begin{array}{l}\text { Method, protocol, } \\
\text { specific analyses }\end{array}$ \\
\hline 1. DST Waste & $\begin{array}{l}1994-1999(M-44-00 \\
\text { Ecology et al. 1992) }\end{array}$ & $\begin{array}{l}\text { - A Tank Waste Analysis Plan is under development using } \\
\text { the results of the data quality objective process for } \\
\text { characterization of all tanks. } \\
\text { Specific analysis will be determined by the data } \\
\text { quality objectives process. } \\
\text { - tank characterization plan will also be developed } \\
\text { using the data quality objectives process. The tank } \\
\text { characterization plans will integrate the results of } \\
\text { the various issue and process efforts into a specific } \\
\text { sampling and aralysis plan for a given tank. }\end{array}$ \\
\hline 2. PUREX Aging Waste & $\begin{array}{l}\text { 1994-1995 (Defense } \\
\text { Nuclear Facilities } \\
\text { Safety Board Commitment } \\
\text { 93-95) }\end{array}$ & $\begin{array}{l}\text { - The number of samples required and sampling methods } \\
\text { will be determined by the data quality objectives } \\
\text { process. }\end{array}$ \\
\hline 3. SST Waste & $1994-1999(M-44-00)$ & $\begin{array}{l}\text { - The number of core samples from each SST will be } \\
\text { determined by the data quality objectives process. } \\
\text { - Samples will be analyzed according to the Tank Waste } \\
\text { Analysis Plan. }\end{array}$ \\
\hline $\begin{array}{l}\text { 4. 242-A Evaporator } \\
\text { Process Condensate }\end{array}$ & $\begin{array}{l}\text { Waste to be sampled in } \\
\text { accordance with } 242-A \\
\text { waste analysis plan }\end{array}$ & $\begin{array}{l}\text { - Future characterization will be negotiated among the } \\
\text { EPA, DOE, and Ecology. } \\
\text { - Treated stream will be characterized after treatment } \\
\text { facility startup. }\end{array}$ \\
\hline $\begin{array}{l}4843 \text { Sodium } \\
\text { Storage Facility } \\
\text { Waste }\end{array}$ & $\begin{array}{l}\text { No future } \\
\text { characterization is } \\
\text { planned }\end{array}$ & 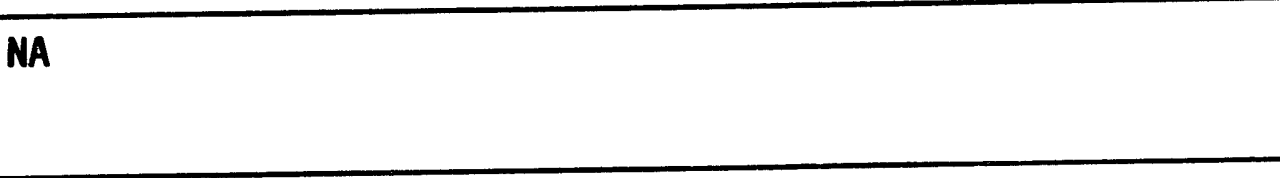 \\
\hline $\begin{array}{l}\text { 6. PUREX Ammonia } \\
\text { Scrubber Waste }\end{array}$ & $\begin{array}{l}\text { 1990-1995, with other } \\
\text { DST waste }\end{array}$ & $\begin{array}{l}\text { - Waste analysis plan completed per M-23-03 (Ecology } \\
\text { et al. 1992). }\end{array}$ \\
\hline $\begin{array}{l}\text { 7. PUREX Process } \\
\text { Condensate }\end{array}$ & $\begin{array}{l}\text { 1990-1995, with other } \\
\text { DST waste }\end{array}$ & $\begin{array}{l}\text { - Waste analysis plan completed per M-23-03 (Ecology } \\
\text { et al. 1992). }\end{array}$ \\
\hline
\end{tabular}


Table 2-2. Haste Stream Characterization. (sheet 2 of 3 )

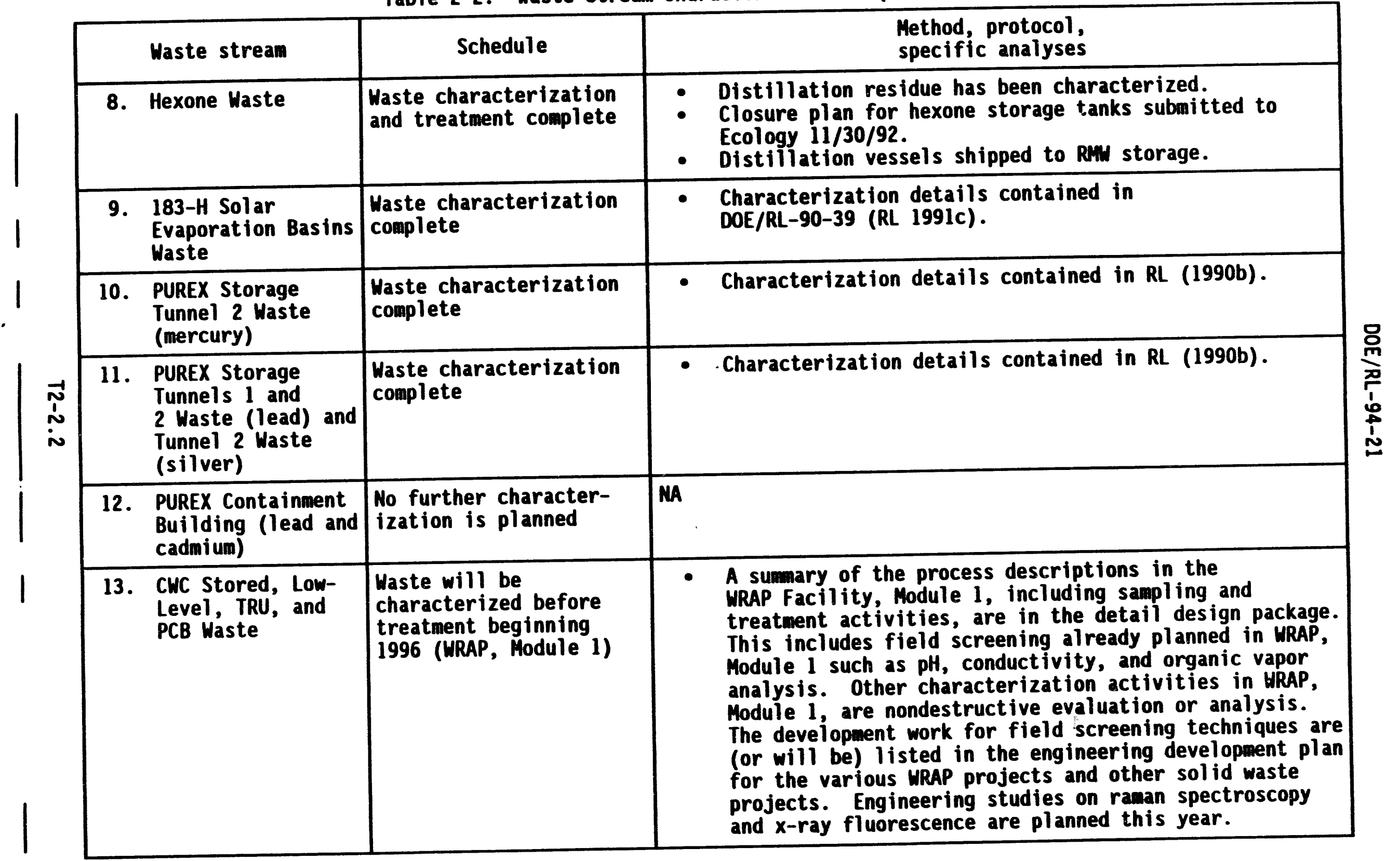


Table 2-2. Waste Stream Characterization. (sheet 3 of 3)

\begin{tabular}{|l|l|l|}
\hline Waste stream & \multicolumn{1}{|c|}{ Schedule } & \multicolumn{1}{c|}{$\begin{array}{c}\text { Method, protocol, } \\
\text { specific analyses }\end{array}$} \\
\hline 14. $\begin{array}{l}\text { Retrievably Stored } \\
\text { Low-Level and TRU } \\
\text { Waste }\end{array}$ & $\begin{array}{l}\text { In situ character- } \\
\text { ization 1991-1994 } \\
\text { Waste will be } \\
\text { characterized } \\
\text { before disposal } \\
\text { after processing } \\
\text { at WRAP Facility }\end{array}$ & $\begin{array}{l}\text { - } \\
\text { Real-time radiography will help identify liquids and } \\
\text { lead in pre-1980 drums. } \\
\text { Gas within containers will be sampled and analyzed to } \\
\text { ascertain whether explosive gas mixtures are present. }\end{array}$ \\
\hline 15. $\begin{array}{l}\text { TRUSAF Stored } \\
\text { Waste }\end{array}$ & $\begin{array}{l}\text { No further character- } \\
\text { ization is planned }\end{array}$ & $\bullet \begin{array}{l}\text { Certified and shipped to the Waste Isolation Pilot } \\
\text { Plant. }\end{array}$ \\
\hline 16. 303-K Stored Waste & $\begin{array}{l}\text { Waste characterization } \\
\text { complete }\end{array}$ & NA \\
\hline
\end{tabular}

1
1
1
0

CWC = Central Waste Complex.

DOE = U.S. Department of Energy.

DST $=$ Double-shell tank.

Ecology $=$ Washington State Department of Ecology.

$E P A=$ U.S. Environmental Protection Agency.

$N A=$ Not applicable.

PCB = Polychlorinated biphenyl.

PUREX = Plutonium-Uranium Extraction (Plant).

RL $=$ U.S. Department of Energy, Richland Operations Office.

SST = Single-shell tank.

TRU = Transuranic.

TRUSAF = Transuranic Waste Storage and Assay Facility.

WRAP = Waste Receiving and Processing (Facility). 
Table 2-3. Hazardous Waste Designations." (sheet 1 of 5 )

\begin{tabular}{|c|c|}
\hline Waste stream & Designated waste code(s) \\
\hline 1. DST Waste & 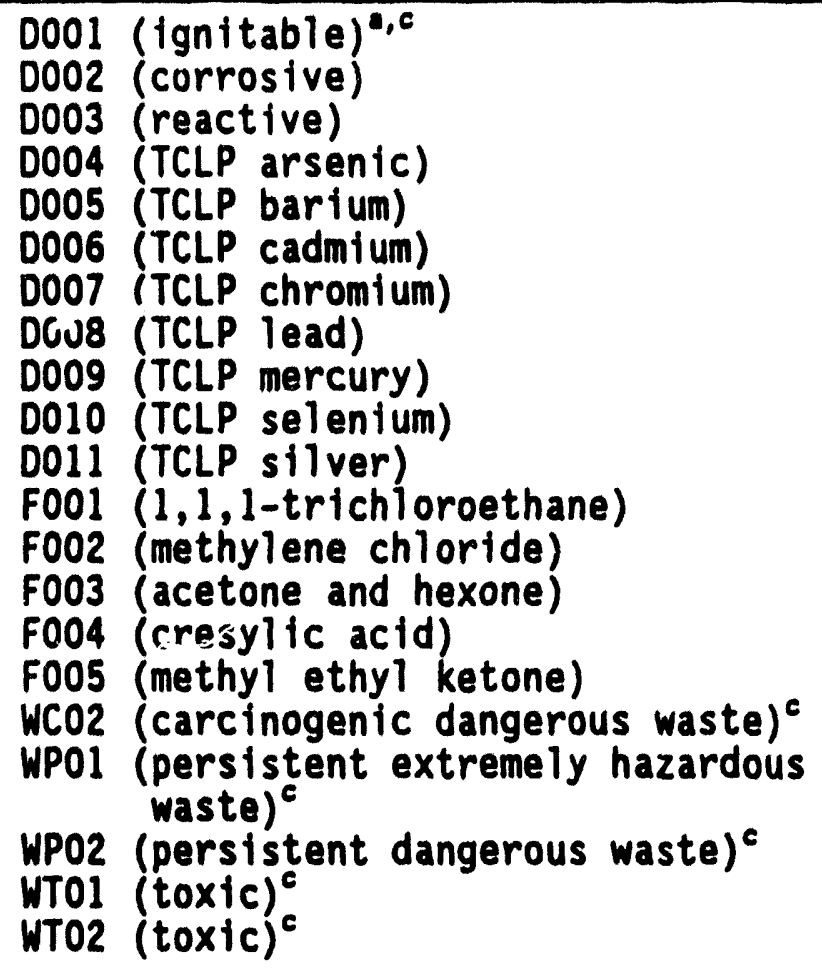 \\
\hline 2. PUREX Aging Waste & $\begin{array}{l}\text { D001 (ignitable)",c } \\
\text { D002 (corrosive) } \\
\text { D006 (TCLP cadmium) } \\
\text { D007 (TCLP chromium) } \\
\text { D008 (TCLP lead) }\end{array}$ \\
\hline 3. SST Waste & $\begin{array}{l}\text { D001 (ignitable) } \\
\text { D002 (corrosive) } \\
\text { D005 (TCLP barium) } \\
\text { D006 (TCLP cadmium) } \\
\text { D007 (TCLP chromium) } \\
\text { D008 (TCLP lead) } \\
\text { D009 (TCLP mercury) } \\
\text { D010 (TCLP selenium) } \\
\text { D011 (TCLP silver) } \\
\text { F003 (acetone and hexone) } \\
\text { F005 (nonspent halogenated solvents) } \\
\text { WT01 (toxic) }\end{array}$ \\
\hline $\begin{array}{l}\text { 4. 242-A Evaporator Process } \\
\text { Condensate }\end{array}$ & $\begin{array}{l}\text { F001 (1,1,1-trichlorethane) } \\
\text { F002 (methylene chloride) } \\
\text { F003 (acetone and hexone) } \\
\text { F004 (cresylic acid) } \\
\text { F005 (methyl ethyl ketone) } \\
\text { WT01 (toxic, extremely hazardous) } \\
\text { WT02 (toxic) }\end{array}$ \\
\hline
\end{tabular}


Table 2-3. Hazardous Waste Designations." (sheet 2 of 5)

\begin{tabular}{|c|c|}
\hline Waste stream & Designated waste code(s) \\
\hline $\begin{array}{l}\text { 5. } 4843 \text { Sodfum Storage Facility } \\
\text { Waste }\end{array}$ & $\begin{array}{l}\text { D001 (ignitable) } \\
\text { D002 (corrosive) } \\
\text { D003 (reactive) } \\
\text { WT01 (toxic) } \\
\text { WT02 (toxic) }\end{array}$ \\
\hline 6. PUREX Ammonia Scrubber Waste & $\begin{array}{l}\text { D002 (corrosive) } \\
\text { WTO1 (toxic) }\end{array}$ \\
\hline 7. PUREX Process Condensate & $\begin{array}{l}\text { D002 (corrosive) } \\
\text { WT02 (toxic) }\end{array}$ \\
\hline 8. Hexone Waste & $\begin{array}{l}\text { D001 (ignitable) } \\
\text { F003 (hexone) } \\
\text { WC02 (carcinogenic) } \\
\text { WT02 (toxic) }\end{array}$ \\
\hline $\begin{array}{l}\text { 9. 183-H Solar Evaporation Basins } \\
\text { Waste }\end{array}$ & $\begin{array}{l}\text { D007 (TCLP chromium) } \\
\text { P029 (copper cyanides) } \\
\text { P030 (soluble cyanide salts) } \\
\text { P098 (potassium cyanide) } \\
\text { P106 (sodium cyanide) } \\
\text { P120 (vanadium pentoxide) } \\
\text { U123 (formic acid) } \\
\text { WT01 (toxic) }\end{array}$ \\
\hline $\begin{array}{l}\text { 10. PUREX Storage Tunnel } 2 \text { Waste } \\
\text { (mercury) }\end{array}$ & $\begin{array}{l}\text { D001 (ignitable) } \\
\text { D009 (TCLP mercury) } \\
\text { D011 (silver nitrate) } \\
\text { WT01 (toxic) }\end{array}$ \\
\hline $\begin{array}{l}\text { 11a. PUREX Storage Tunnels } 1 \text { and } 2 \\
\text { Waste (lead) }\end{array}$ & $\begin{array}{l}\text { D008 (TCLP lead) } \\
\text { WT01 (toxic) }\end{array}$ \\
\hline $\begin{array}{l}\text { 11b. PUREX Storage Tunnel } 2 \text { Waste } \\
\text { (silver) }\end{array}$ & $\begin{array}{l}\text { D001 (ignitable) } \\
\text { D011 (TCLP silver) } \\
\text { WTO1 (toxic) }\end{array}$ \\
\hline $\begin{array}{l}\text { 12. PUREX Containment Building } \\
\text { (1ead and cadmium) }\end{array}$ & $\begin{array}{l}\text { D006 (TCLP cadmium) } \\
\text { D008 (TCLP lead) } \\
\text { WT01 (toxic) }\end{array}$ \\
\hline
\end{tabular}


Table 2-3. Hazardous Waste Designations. (sheet 3 of 5)

\begin{tabular}{|c|c|c|}
\hline & Waste stream & Destgnated waste code(s) \\
\hline 13. & $\begin{array}{l}\text { CWC Stored Low-Level, TRU, and } \\
\text { PCB Waste } \\
\text { NOTE: Due to the nature of } \\
\text { this factlity, an extensive } \\
\text { number of waste codes apply. } \\
\text { Some of the major codes are } \\
\text { presented here. (This also } \\
\text { applies to Tabie 2-6.) The } \\
\text { Part A permit application } \\
\text { contains a complete listing. }\end{array}$ & $\begin{array}{l}\text { D001 (tgnitable) } \\
\text { D002 (corrosive) } \\
\text { D003 (reactive) } \\
\text { D004 (TCLP arsenic) } \\
\text { D005 (TCLP barium) } \\
\text { D006 (TCLP Cadmium) } \\
\text { D007 (TCLP chromium) } \\
\text { D008 (TCLP lead) } \\
\text { D009 (TCLP mercury) } \\
\text { D010 (TCLP selenium) } \\
\text { D011 (TCLP silver) } \\
\text { D012 (TCLP Endrin) } \\
\text { D016 (TCLP 2, 4-D) } \\
\text { F001 (spent halogenated degreasing } \\
\text { F002 (spent halogenated solvents) } \\
\text { F003 (acetone) } \\
\text { F004 (cresols) } \\
\text { F005 (spent non-halogenated solvents) } \\
\text { P029 (copper cyanides) } \\
\text { P030 (soluble cyanide salts) } \\
\text { P098 (potassium cyanide) } \\
\text { P106 (sodium cyanide) } \\
\text { P120 (vanadium pentoxide) } \\
\text { U080 (dichloromethane) } \\
\text { U123 (formic acid) } \\
\text { U161 (methylisobutylketone) } \\
\text { W001 (PCBs) } \\
\text { WC02 (carcinogenic dangerous waste) } \\
\text { WP01 (persistent extremely hazardous } \\
\text { WP02 (waste) } \\
\text { WT01 (toxic) } \\
\text { WT02 (toxic) }\end{array}$ \\
\hline
\end{tabular}


Table 2-3. Hazardous Waste Designations.' (sheet 4 of 5)

\begin{tabular}{|c|c|}
\hline Waste stream & Designated waste code(s) \\
\hline $\begin{array}{l}\text { 14. Retrievably Stored Low-Level } \\
\text { and TRU Waste }\end{array}$ & 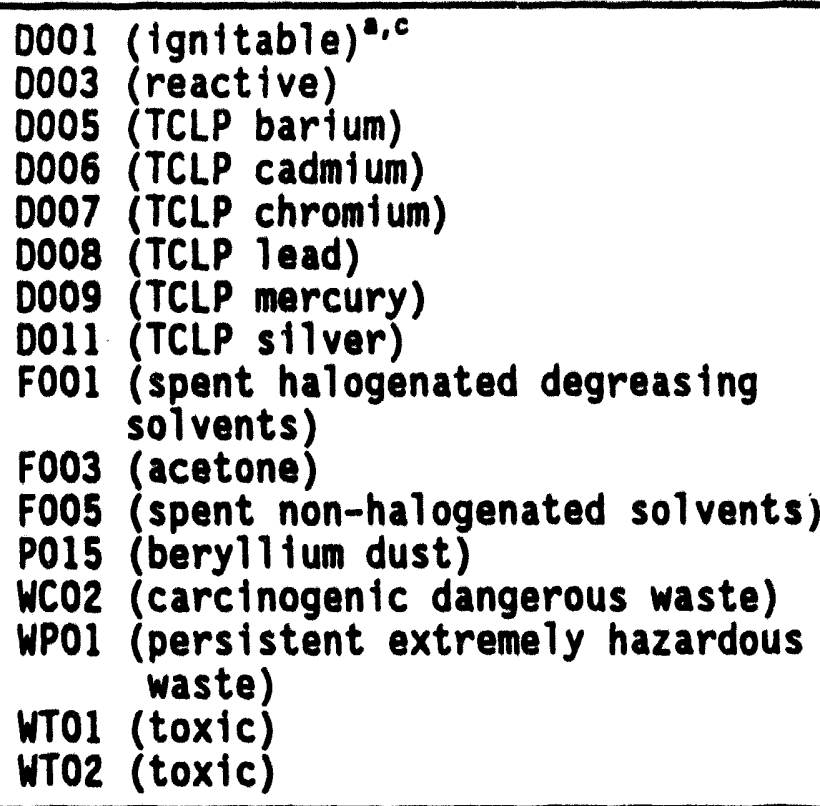 \\
\hline 15. TRUSAF Stored Waste & $\begin{array}{l}\text { D002 (corrosive) } \\
\text { D005 (TCLP barium) } \\
\text { D006 (TCLP cadmium) } \\
\text { D007 (TCLP chromium) } \\
\text { D008 (TCLP lead) } \\
\text { D009 (TCLP mercury) } \\
\text { WCO2 (carcinogenic dangerous waste) } \\
\text { WP01 (persistent extremely hazardous } \\
\text { WTaste) } \\
\text { WT01 (toxic) }\end{array}$ \\
\hline
\end{tabular}


Table 2-3. Hazardous Waste Designattons." (sheet 5 of 5)

\begin{tabular}{|c|c|}
\hline Waste stream & Designated waste code(s) \\
\hline 16. 303-K Stored Waste & $\begin{array}{l}\text { D001 (ignitable) } \\
\text { D006 (TCLP cadmium) } \\
\text { D007 (TCLP chromium) } \\
\text { D008 (TCLP lead) } \\
\text { D039 (perchiorethylene) } \\
\text { F001 (spent halogenated degreasing } \\
\text { solvents) } \\
\text { F002 (spent halogenated solvents) } \\
\text { WC02 (carcinogenic) } \\
\text { WP01 (persistent) } \\
\text { WT01 (toxic) }\end{array}$ \\
\hline
\end{tabular}

Further information is given in Section 2.2 .

BTCLP waste codes D018, D019, D022, D028, D029, D030, D033, D035, D036, and 0038 through D043 are listed in the DST Part A Permit appl lcation but are not listed in this table or in Table 2-6 because analysis of tank waste has not yet confirmed these to be present.

'Designation is based on process knowledge; waste has not been laboratory analyzed for these components.

This waste has been removed and transferred to the CWC (waste strean 13 in this report).

CuC = Central Waste Complex.

DST - Double-shell tank.

PCB = Polychlorinated biphanyl.

PUREX = Plutonium-Uranium Extraction (Plant).

SST = Single-shell tank.

TCLP = Toxic characteristic leach procedure.

TRU = Transuranic.

TRUSAF = Transuranic Waste Storage and Assay Facility. 
Table 2-4. Storage Unit Characteristics. (sheet 1 of 2)

\begin{tabular}{|c|c|c|c|c|c|}
\hline Wate streen & fecllity & cepecity $\left(0^{3}\right)$ & $\begin{array}{l}\text { Anticipated } \\
\text { capecity fill } \\
\text { date }\end{array}$ & $\begin{array}{c}\text { Part } \\
\text { Declosure } \\
\text { Plen (Letes; } \\
\text { Revision) }\end{array}$ & $\begin{array}{l}\text { thoun Rel case of } \\
\text { harardori } \\
\text { constituents }\end{array}$ \\
\hline 1. DST Haste & DSTs & 111,800 & 1924 & $6 / 91$ & nom \\
\hline 2. Priex Aging Haste & OSTs & 7,400 & 1989 & $6 / 91$ & nome \\
\hline 3. SSt waste & SST: & $357.300^{\circ}$ & $m^{b}$ & $9 / 00^{6}$ & $y=(T a b l e$ 3-6) \\
\hline 4. 262-A Eveporator Process Condmante & LERF & 49,000 & 1995 & $6 / 91$ & nome \\
\hline 5. 4043 sodiun storace Fecillity Uate & 4863 vulldine & $86,000 \mathrm{~kg}$ & $m^{b}$ & $691^{c}$ & nome \\
\hline 6. Preex amonie scrubber Maste & DSTs & 111,000 & $m^{b}$ & $6 / 91$ & nome \\
\hline 7. Punex Process condensate & DSTs & 111,000 & $m^{b}$ & $6 / 91$ & $\operatorname{nom}$ \\
\hline 8. Mexone Mate & $\begin{array}{l}276-5-141 \\
276-5-142\end{array}$ & $170^{9}$ & $m^{b}$ & $11 / 92^{c}$ & $\operatorname{nom}$ \\
\hline 9. 183-11 Solar Eveporation Easins Maste & 183-n casins & $8,200^{\circ}$ & $m^{b}$ & $691^{c}$ & $r e$ (Section 3.9.3) \\
\hline $\begin{array}{l}\text { 10. Punex storese Tumal } 2 \text { Maste } \\
\text { (eercury) }\end{array}$ & Puncx Tunnal 2 & d & $m^{e}$ & $12 / 91^{f}$ & nome \\
\hline $\begin{array}{l}\text { 11. Puasx storece Tumals } 1 \text { and } 2 \text { Uaste } \\
\text { (lead) and Tumal } 2 \text { Waste (si lver) }\end{array}$ & puasex tumals 1, 2 & d & $m^{2}$ & $12 \mu 1^{t}$ & $\operatorname{non}$ \\
\hline $\begin{array}{l}\text { 12. Punex contoinment cuilding (lead and } \\
\text { cadbivin) }\end{array}$ & puncx campon & b & $m^{*}$ & $\begin{array}{l}\text { Clome plen } \\
\text { Two }\end{array}$ & none \\
\hline $\begin{array}{l}\text { 13. Cuc Stored Lou-Level, TRu, and PCS } \\
\text { Haste }\end{array}$ & Verion & 23,093 & 1999 & $10 / 91$ & $\operatorname{man}$ \\
\hline $\begin{array}{l}\text { 16. Retrievably Stored Lou-Level and Tay } \\
\text { Uastes }\end{array}$ & Various & $15,460^{\circ}$ & $m^{n}$ & $10 / 91$ & nom \\
\hline
\end{tabular}


Table 2-4. Storage Unit Characteristics. (sheet 2 of 2)

\begin{tabular}{|c|c|c|c|c|c|}
\hline weste streen & facillity & capecity (13) & $\begin{array}{l}\text { Anticipated } \\
\text { cepacity fill } \\
\text { dote }\end{array}$ & $\begin{array}{c}\text { Part } \\
\text { erclowre } \\
\text { Plen (Lotest }\end{array}$ & $\begin{array}{l}\text { thoun Release of } \\
\text { hazardous } \\
\text { constituents }\end{array}$ \\
\hline 15. Trusaf stored mate & 226-T Eullding & 420 & $m^{5}$ & $6 / 92$ & neme \\
\hline 16. 303-K stored wate & 303-K Dullding & 42 & $m^{0}$ & $12 / 9 s^{c}$ & neme \\
\hline
\end{tabular}

This unit is no loner uned for ective storese; ceppecity noted is for informtion only.

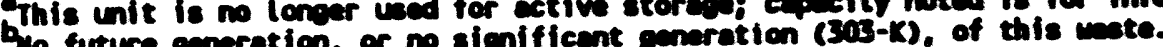

Gio future ganer

cloeure plen.

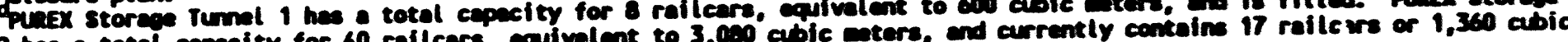

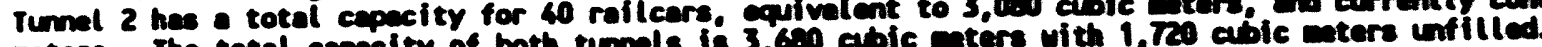

meters. The total cepecity of bot all future emeration.

To be cheread to a clowure plen.

ar = Central Laste Complex.

DST = Double-ahall tenk.

LERF = Liquid Effluent Retention Facillity.

$m a$ = Not eoplicable.

PCS = Polychlorinated biphent.

PDEx = Plutoniu-Ureniv Extraction (fecility).

SST = sinole-chell tenk.

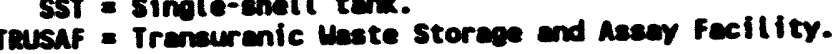


Table 2-5. Stored Waste Characteristics. (sheet 1 of 2)

\begin{tabular}{|c|c|c|c|c|c|c|c|c|c|}
\hline Maste etrecm & facility & $\begin{array}{l}\text { mount in } \\
\text { storase }\left(n^{3}\right)\end{array}$ & $\begin{array}{l}\text { Date first } \\
\text { waste in } \\
\text { sterage }\end{array}$ & $\underset{(x)}{\text { Liquid }}$ & $\begin{array}{l}\text { Solid } \\
(x)\end{array}$ & $\begin{array}{l}\text { sludes } \\
(x)\end{array}$ & (x) & $\underset{(x)}{T R U / L L H}$ & $\begin{array}{l}\text { HLW } \\
(x)\end{array}$ \\
\hline 1. DST Uaste & DSTs & $89,756^{\circ}$ & 1975 & 80 & 11 & 9 & 6 & $\mathbf{b}$ & $\mathbf{b}$ \\
\hline 2. PunEX Agino Uaste & DSTs & $7.211^{\circ}$ & 1975 & 93 & 0 & 7 & 0 & 0 & 100 \\
\hline 3. Sst Maste & SST & 139,500 & 1946 & 31 & 44 & 25 & $b$ & $b$ & b \\
\hline 4. 242-A Eveporator Process Condenente & LEAf & nome & & 100 & 0 & 0 & 100 & 0 & 0 \\
\hline 5. 4843 sodiu storage fecillity Maste & 4843 Building & 8.5 & $19 a 7$ & 0 & 100 & 0 & 100 & 0 & 0 \\
\hline 6. PuREX ammonie scrubber Maste & DSTs & $5.900^{\circ}$ & 1907 & 100 & 0 & 0 & 100 & 0 & 0 \\
\hline 7. PUREX Process Condensate & DSTs & $6,800^{\circ}$ & 1909 & 100 & 0 & 0 & 100 & 0 & 0 \\
\hline 8. Mexone Waste & $276-5-141,142$ & 0.4 & 1951 & 8 & 0 & 92 & 100 & 0 & 0 \\
\hline 9. 183-H Solar Eveporation Basins Maste & 183-n Dasins & $\operatorname{none}^{c}$ & 1973 & $20^{c}$ & $80^{c}$ & 0 & $100^{c}$ & 0 & 0 \\
\hline $\begin{array}{l}\text { 10. PunEX Storage Tumel } 2 \text { Uaste } \\
\text { (mercury) }\end{array}$ & Punex Tunnel 2 & $0.01^{d}$ & 1971 & 100 & 0 & 0 & 100 & $\mathbf{0}$ & 0 \\
\hline $\begin{array}{l}\text { 11. PUREX Storage Tumels } 1 \text { and } 2 \text { Waste } \\
\text { (lead) }\end{array}$ & $\begin{array}{l}\text { PUREX Tumal } 1 \\
\text { PUREX Turnal } 2\end{array}$ & $0.26^{d}$ & 1960 & 0 & 100 & 0 & 100 & 0 & 0 \\
\hline $\begin{array}{l}\text { 11b. PuREX storage Tumal } 2 \text { Maste } \\
\text { (silver) }\end{array}$ & PUREX TUnml 2 & $0.17^{d}$ & 1971 & 0 & 100 & 0 & 100 & 0 & 0 \\
\hline $\begin{array}{l}\text { 12. PuREX Containment Building (lead and } \\
\text { cactaiu) }\end{array}$ & PUREX Plant & $0.29^{d}$ & 1987 & 0 & 100 & 0 & 100 & 0 & 0 \\
\hline $\begin{array}{l}\text { 13. Cuc Stored Lou-Level, TRU, and PCB } \\
\text { Hastes }\end{array}$ & Various & 5.118 & 1988 & 0 & 100 & 0 & 95 & 5 & 0 \\
\hline $\begin{array}{l}\text { 16. Retrievebly Stored Lou-Level and TRU } \\
\text { Wostes }\end{array}$ & Verious & 2,184 & 1970 & 0 & 100 & 0 & 78 & 22 & 0 \\
\hline
\end{tabular}


Table 2-5. Stored Waste Characteristics. (sheet 2 of 2)

\begin{tabular}{|c|c|c|c|c|c|c|c|c|c|}
\hline Waste strean & Fecilfty & $\begin{array}{l}\text { anount in } \\
\text { storage }\left(n^{3}\right)\end{array}$ & $\begin{array}{l}\text { Date first } \\
\text { waste in } \\
\text { storege }\end{array}$ & $\underset{(x)}{\text { Liquid }}$ & $\begin{array}{l}\text { Solid } \\
(x)\end{array}$ & $\underset{(X)}{\text { sludee }}$ & $\begin{array}{l}\text { LLW } \\
(x)\end{array}$ & $\begin{array}{c}\text { TRU/LLU } \\
(x)\end{array}$ & $\begin{array}{l}M L H \\
(x)\end{array}$ \\
\hline 15. Tmusaf stored Uaste & $224-1 \mathrm{Dlda}$ & 43 & 1905 & $\mathbf{0}$ & 100 & $\mathbf{0}$ & 0 & 100 & $\mathbf{0}$ \\
\hline 16. 303-K stored Haste & $303-K$ eldo & 5.5 & 1943 & 0 & 100 & $\mathbf{0}$ & 100 & 0 & $\mathbf{0}$ \\
\hline
\end{tabular}

a Imventories for PUREX Amonia Scrubber Maste and PUaEX Process Condeneate elso are included in the DST Uaste immentory. PUnEX Aging Maste is (is 96,967 cubic esters.

ipcluded in the DST Maste imventory. The total DST Waste inmentory is 9s,

Penk waste contains LLW, TRU, and MLW. Nowever. in the interiw storres

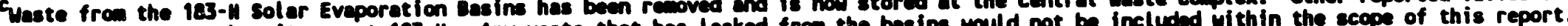

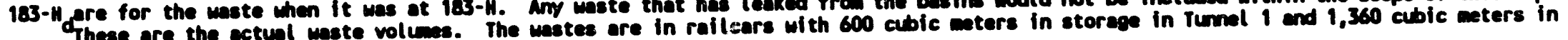
storage in Tunnel 2 (roilcars included).

\section{Cuc = Central Maste Complex.}

DST = Double-shell tenk.

IIU = Mich-level uaste.

LERF = Liquid Effluent Retention Fecility.

LERF = Llquid Effluent

LLE = Low-level maste.

PCB = Plutoniu-Ureniu Extraction (Fecility).

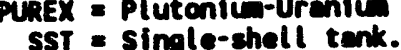

TRU = Trensurenic (maste).

TRUSAF = Transuranic Haste Storage and Aseay facility. 
Table 2-6. Treatment of Land Disposal Restricted Waste for Disposal. (sheet 1 of 10)

\begin{tabular}{|c|c|c|c|c|c|c|}
\hline $\begin{array}{l}\text { Wastes } \\
\text { codes }\end{array}$ & $\begin{array}{l}\text { Required } \\
\text { treatment }\end{array}$ & $\begin{array}{l}\mathrm{Pl} \text { anned } \\
\text { treatment }\end{array}$ & $\begin{array}{l}\text { Treatment } \\
\text { facility }\end{array}$ & $\begin{array}{l}\text { Facility } \\
\text { capacity } \\
\text { (m/day) }\end{array}$ & $\begin{array}{l}\text { Disposal } \\
\text { facility }\end{array}$ & $\begin{array}{l}\text { Treat- } \\
\text { ment } \\
\text { date }\end{array}$ \\
\hline \multicolumn{7}{|c|}{$\begin{array}{l}\text { 1. DST Waste (includes NCAW, NCRW, complex concentrate, and PFP waste) } \\
\text { (low-level fraction) }\end{array}$} \\
\hline F001 & CCW & vitrification & $\mathrm{TBD}^{\mathrm{C}}$ & TBD & TBD(onsite) & 2005 \\
\hline F002 & $\mathrm{CCH}$ & vitrification & TBDC & $\mathrm{TBD}^{d}$ & TBD(onsite; & 2005 \\
\hline F003 & $\mathrm{CCH}$ & vitrification & $\mathrm{TBO}^{\circ}$ & $\mathrm{TBO}^{\mathrm{d}}$ & TBD(onsite) & 2005 \\
\hline F004 & $\mathrm{CCH}$ & vitrification & $\mathrm{TBD}^{\mathrm{C}}$ & $T B D^{d}$ & TBD(onsite) & 2005 \\
\hline F005 & $\mathrm{CCW}$ & vitrification & $\mathrm{TBD}^{\mathrm{C}}$ & $\mathrm{TBD}^{\mathrm{d}}$ & TBD(onsite) & 2005 \\
\hline D001 & deactivation & vitrification & $\mathrm{TBD}^{\mathrm{C}}$ & $\mathrm{TBD}^{\mathrm{d}}$ & TBD(onsite) & 2005 \\
\hline 0002 & deactivation & vitrification & $T B D^{C}$ & $T_{B D}^{d}$ & TBD(onsite) & 2005 \\
\hline D003 & deactivation & vitrification & $\mathrm{TBD}^{\mathrm{C}}$ & $\mathrm{TBD}^{\mathrm{d}}$ & TBD(onsite) & 2005 \\
\hline D004 & CCWE & vitrification & $T B D^{C}$ & $\mathrm{TBO}^{\mathrm{d}}$ & TBD(onsite) & 2005 \\
\hline D005 & CCWE & vitrification & $T_{B D}{ }^{C}$ & $\mathrm{TBD}^{\mathrm{d}}$ & TBD(onsite) & 2005 \\
\hline D006 & CCWE & vitrification & $\mathrm{TBD}^{\mathrm{C}}$ & $\mathrm{TBD}^{\mathrm{d}}$ & TBD(onsite) & 2005 \\
\hline D007 & CCWE & vitrification & $\mathrm{TBD}^{\mathrm{C}}$ & $\mathrm{TBD}^{\mathrm{d}}$ & TBD(onsite) & 2005 \\
\hline D008 & CCWE & vitrification & $\mathrm{TBD}^{\mathrm{C}}$ & $T B D^{d}$ & TBD(onsite) & 2005 \\
\hline 0009 & CCWE & vitrification & $T_{B D}{ }^{C}$ & $T_{B D}^{d}$ & TBD(onsite) & 2005 \\
\hline D010 & CCWE & vitrification & $\mathrm{TBO}^{\mathrm{C}}$ & $T B D^{d}$ & TBD(onsite) & 2005 \\
\hline D011 & CCWE & vitrification & $\mathrm{TBD}^{\mathrm{C}}$ & $T B D^{d}$ & TBD(onsite) & 2005 \\
\hline WT01 & reduction & vitrification & $\mathrm{TBD}^{\mathrm{C}}$ & $\mathrm{TBO}^{\mathrm{d}}$ & TBD(onsite) & 2005 \\
\hline WTO2 & none & vitrification & $\mathrm{TBD}^{\mathrm{C}}$ & TBOd & TBD(onsite) & 2005 \\
\hline WCO2 & none & vitrification & $\mathrm{TBO}^{\mathrm{C}}$ & $T B D^{d}$ & TBD(onsite) & 2005 \\
\hline WPO1 & reduction & vitrification & $\mathrm{TBD}^{\mathrm{C}}$ & $\mathrm{TBD}^{\mathrm{d}}$ & TBD(onsite) & 2005 \\
\hline WP02 & none & vitrification & $T B D^{C}$ & $\mathrm{TBD}^{\mathrm{d}}$ & TBD(onsite) & 2005 \\
\hline \multicolumn{7}{|c|}{ Pretreated Complexed Concentrate Waste (high-level fraction) } \\
\hline F001 & $\mathrm{CCH}$ & vitrification & TBD' & TBD & repository & 2009 \\
\hline F002 & $\mathrm{CCH}$ & vitrification & $\mathrm{TBO}^{\mathrm{C}}$ & TBD & repository & 2009 \\
\hline F003 & $\mathrm{CCH}$ & vitrification & $\mathrm{TBO}^{\mathrm{C}}$ & TBD & repository & 2009 \\
\hline F004 & $\mathrm{CCH}$ & vitrification & $\mathrm{TBO}^{\mathrm{C}}$ & TBD & repository & 2009 \\
\hline F005 & CCW & vitrification & TBDC & TBD & repository & 2009 \\
\hline
\end{tabular}


Table 2-6. Treatment of Land Disposal Restricted Waste for Disposal. (sheet 2 of 10)

\begin{tabular}{|c|c|c|c|c|c|c|}
\hline $\begin{array}{c}\text { Wastes } \\
\text { codes }\end{array}$ & $\begin{array}{l}\text { Required } \\
\text { treatment }\end{array}$ & $\begin{array}{l}\text { Planned } \\
\text { treatment }\end{array}$ & $\begin{array}{l}\text { Treatment } \\
\text { facility }\end{array}$ & $\begin{array}{l}\text { Facility } \\
\text { capacity } \\
\left(\mathrm{m}^{3} / \text { day }\right)\end{array}$ & $\begin{array}{l}\text { Disposal } \\
\text { facility }\end{array}$ & $\begin{array}{c}\text { Treat- } \\
\text { ment } \\
\text { date }\end{array}$ \\
\hline $\mathrm{D} 001$ & deactivation & vitrification & $T_{B D}{ }^{C}$ & TBD & repository & 2009 \\
\hline D002 & deactivation & vitrification & $\mathrm{TBD}^{\mathrm{C}}$ & TBD & repository & 2009 \\
\hline D003 & deactivation & vitrification & $T B D^{C}$ & TBD & repository & 2009 \\
\hline D004 & CCHE & vitrification & $\mathrm{TBD}^{\mathrm{C}}$ & TBD & repository & 2009 \\
\hline D005 & CCHE & vitrification & $\mathrm{TBD}^{\mathrm{C}}$ & TBD & repository & 2009 \\
\hline D006 & CCWE & vitrification & $\mathrm{TBD}^{\mathrm{C}}$ & TBD & repository & 2009 \\
\hline D007 & CCWE & vitrification & TBD & TBD & repository & 2009 \\
\hline 0008 & CCWE & vitrification & TBD ${ }^{C}$ & TBD & repository & 2009 \\
\hline 0009 & CCWE & vitrification & $\mathrm{TBO}^{\mathrm{C}}$ & TBD & repository & 2009 \\
\hline 0010 & CCWE & vitrification & $T_{B D}{ }^{C}$ & TBD & repository & 2009 \\
\hline D011 & CCWE & vitrification & $T B D^{C}$ & TBD & repository & 2009 \\
\hline WT01 & reduction & vitrification & $\mathrm{TBD}^{\mathrm{C}}$ & TBD & repository & 2009 \\
\hline WTO2 & none & vitrification & TBDC & TBD & repository & 2009 \\
\hline WCO2 & none & vitrification & $\mathrm{TBO}^{\mathrm{C}}$ & TBD & repository & 2009 \\
\hline WPO1 & reduction & vitrification & $\mathrm{TBD}^{\mathrm{C}}$ & TBD & repository & 2009 \\
\hline WPO2 & none & vitrification & $\mathrm{TBD}^{\mathrm{C}}$ & TBD & repository & 2009 \\
\hline \multicolumn{7}{|c|}{$\begin{array}{l}\text { 2. PUREX Aging Waste } \\
\text { High-level fraction }\end{array}$} \\
\hline D001 & deactivation & vitrification & $\mathrm{TBD}^{\mathrm{C}}$ & TBD & repository & 2009 \\
\hline 0002 & vitrification & vitrification & TBDC & TBD & repository & 2009 \\
\hline 0006 & vitrification & vitrification & $\mathrm{TBD}^{\mathrm{C}}$ & TBD & repository & 2009 \\
\hline D007 & vitrification & vitrification & $T B D^{C}$ & TBD & repository & 2009 \\
\hline D008 & vitrification & vitrification & $\mathrm{TBO}^{\mathrm{C}}$ & TBD & repository & 2009 \\
\hline \multicolumn{7}{|c|}{ Low-level fraction } \\
\hline D001 & deactivation & Vitrification & $\mathrm{TBD}^{\mathrm{C}}$ & $\mathrm{TBD}^{\mathrm{d}}$ & TBD (onsite) & 2009 \\
\hline 0002 & deactivation & Vitrification & TBDC & $\mathrm{TBD}^{\mathrm{d}}$ & TBD (onsite) & 2009 \\
\hline D006 & CCWE & Vitrification & $T_{B D}{ }^{C}$ & $T_{B D}^{d}$ & TBD (onsite) & 2009 \\
\hline 0007 & CCWE & Vitrification & $\mathrm{TBO}^{\mathrm{C}}$ & $T B D^{d}$ & TBD (onsite) & 2009 \\
\hline 0008 & CCWE & Vitrification & TBDC & $\mathrm{TBD}^{\mathrm{d}}$ & TBD (onsite) & 2009 \\
\hline
\end{tabular}


Table 2-6. Treatment of Land Disposal Restricted Waste for Disposal. (sheet 3 of 10)

\begin{tabular}{|c|c|c|c|c|c|c|}
\hline $\begin{array}{l}\text { itesites } \\
\text { des des }\end{array}$ & $\begin{array}{l}\text { Required } \\
\text { treatment }\end{array}$ & $\begin{array}{l}\text { Planned } \\
\text { treatment }\end{array}$ & $\begin{array}{l}\text { Treatment } \\
\text { facility }\end{array}$ & $\begin{array}{l}\text { Facility } \\
\text { capacity } \\
\left(\mathrm{m}^{3} / \text { day }\right)\end{array}$ & $\begin{array}{l}\text { Disposal } \\
\text { facility }\end{array}$ & $\begin{array}{c}\text { Treat- } \\
\text { ment } \\
\text { date }\end{array}$ \\
\hline \multicolumn{7}{|c|}{$\begin{array}{l}\text { 3. SST Waste } \\
\text { High-level fraction }\end{array}$} \\
\hline 33 & $\mathrm{CCH}$ & vitrification & $\mathrm{TBD}^{\mathrm{C}}$ & TBD & repository & 2009 \\
\hline $5 \quad 5$ & $\mathrm{CCH}$ & vitrification & $\mathrm{TBD}^{\mathrm{C}}$ & TBD & repository & 2009 \\
\hline 111 & deactivation & vitrification & TBD' & TBD & repository & 2009 \\
\hline 212 & deactivation & vitrification & TBDC & TBD & repository & 2009 \\
\hline 1515 & CCWE & vitrification & TBDC & TBD & repository & 2009 \\
\hline $16 \quad 16$ & CCWE & vitrification & TBDC & TBD & repository & 2009 \\
\hline $17 \quad 17$ & CCWE & vitrification & TBDC & TBD & repository & 2009 \\
\hline $18 \quad 18$ & CCWE & vitrification & TBDC & TBD & repository & 2009 \\
\hline $19 \quad 19$ & CCWE & vitrification & TBDC & TBD & repository & 2009 \\
\hline 1010 & CCWE & vitrification & TBDC & TBD & repository & 2009 \\
\hline 1111 & CCWE & vitrification & $\mathrm{TBD}^{\mathrm{C}}$ & TBD & repository & 2009 \\
\hline \multicolumn{7}{|c|}{ Low-level fraction } \\
\hline $03 \quad 03$ & $\mathrm{CCW}$ & vitrification & $\mathrm{TBD}^{\mathrm{C}}$ & $\mathrm{TBD}^{\mathrm{d}}$ & TBD (onsite) & 2005 \\
\hline $05 \quad 05$ & $\mathrm{CCH}$ & vitrification & $\mathrm{TBO}^{\mathrm{C}}$ & $\mathrm{TBD}^{\mathrm{d}}$ & TBD (onsite) & 2005 \\
\hline 0101 & deactivation & vitrification & TBDC & $\mathrm{TBO}^{\mathrm{d}}$ & TBD (onsite) & 2005 \\
\hline 0202 & deactivation & vitrification & $\mathrm{TBD}^{\mathrm{C}}$ & TBD $^{d}$ & TBD (onsite) & 2005 \\
\hline 0505 & CCWE & vitrification & TBD' & $\mathrm{TBO}^{\mathrm{d}}$ & TBD (onsite) & 2005 \\
\hline $06 \quad 06$ & CCWE & vitrification & $\mathrm{TBD}^{\mathrm{C}}$ & TBO $^{d}$ & TBD (onsite) & 2005 \\
\hline $07 \quad 07$ & CCWE & vitrification & TBDC & $\mathrm{TBO}^{\mathrm{d}}$ & TBD (onsite) & 2005 \\
\hline $08 \quad \overline{08}$ & CCWE & vitrification & $T B D^{C}$ & $T_{B D}^{d}$ & TBD (onsite) & 2005 \\
\hline 109109 & CCWE & vitrification & TBDC & $\mathrm{TBD}^{\mathrm{d}}$ & TBD (onsite) & 2005 \\
\hline $110 \quad 110$ & CCWE & vitrification & TBDC & $\mathrm{TBD}^{\mathrm{d}}$ & TBD (onsite) & 2005 \\
\hline 111111 & CCWE & vitrification & TBDC & $\mathrm{TBD}^{\mathrm{d}}$ & TBD (onsite) & 2005 \\
\hline \multicolumn{7}{|c|}{ 4. 242-A Evaporator Process Condensate } \\
\hline 101101 & $\mathrm{CCH}$ & destruction & ETF & TBD & SALDS & 1995 \\
\hline $102 \quad 02$ & $\mathrm{CCH}$ & destruction & ETF & TBD & SALDS & 1995 \\
\hline 203503 & $\mathrm{CCH}$ & destruction & ETF & TBD & SALDS & 1995 \\
\hline
\end{tabular}


Table 2-6. Treatment of Land Disposal Restricted Waste for Disposal. (sheet 4 of 10)

\begin{tabular}{|c|c|c|c|c|c|c|}
\hline $\begin{array}{l}\text { Wastes } \\
\text { codes }\end{array}$ & $\begin{array}{l}\text { Required } \\
\text { treatment }\end{array}$ & $\begin{array}{l}\text { Planned } \\
\text { treatment }\end{array}$ & $\begin{array}{l}\text { Treatment } \\
\text { facility }\end{array}$ & $\begin{array}{l}\text { Facility } \\
\text { capacity } \\
\left(m^{3} / \text { day }\right)\end{array}$ & $\begin{array}{l}\text { Disposal } \\
\text { facility }\end{array}$ & $\begin{array}{l}\text { Treat- } \\
\text { ment } \\
\text { date }\end{array}$ \\
\hline F005 & $\mathrm{CCW}$ & destruction & ETF & TBD & SALDS & 1995 \\
\hline WT02 & none & removal & ETF & TBD & SALDS & 1995 \\
\hline \multicolumn{7}{|c|}{ 5. 4843 Sodium Storage Facility Waste } \\
\hline 0001 & deactivation & deactivation & TBD & TBD & LLBG & TBD \\
\hline D002 & deactivation & deactivation & TBD & TBD & LLBG & TBD \\
\hline D003 & deactivation & deactivation & TBD & TBD & LLBG & TBD \\
\hline WT01 & reduction & TBD & TBD & TBD & TBD & TBD \\
\hline WT02 & none & TBD & TBD & TBD & TBD & TBD \\
\hline \multicolumn{7}{|c|}{ 6. PUREX Ammonia Scrubber Waste } \\
\hline \multicolumn{7}{|c|}{ Included with LLW DST wastes. } \\
\hline \multicolumn{7}{|c|}{ 7. PUREX Process Condensate } \\
\hline \multicolumn{7}{|c|}{ Included with LLW DST wastes. } \\
\hline \multicolumn{7}{|c|}{ 8. Hexone Waste. } \\
\hline F003 & $\mathrm{CCH}$ & incineration & $\begin{array}{l}\text { Diversified } \\
\text { Scientific } \\
\text { Services, } \\
\text { Kingston, TN }\end{array}$ & 12 & $\begin{array}{l}\text { None } \\
\text { (complete } \\
\text { destruc- } \\
\text { tion) }\end{array}$ & $\begin{array}{l}1991- \\
1993\end{array}$ \\
\hline WTO2 & none & incineration & $\begin{array}{l}\text { Diversified } \\
\text { Scientific } \\
\text { Services, } \\
\text { Kingston, TN }\end{array}$ & 12 & $\begin{array}{l}\text { None } \\
\text { (complete } \\
\text { destruc- } \\
\text { tion) }\end{array}$ & $\begin{array}{l}1991- \\
1993\end{array}$ \\
\hline 0001 & deactivation & incineration & $\begin{array}{l}\text { Diversified } \\
\text { Scientific } \\
\text { Services, } \\
\text { Kingston, TN }\end{array}$ & 12 & $\begin{array}{l}\text { None } \\
\text { (complete } \\
\text { destruc- } \\
\text { tion) }\end{array}$ & $\begin{array}{l}1991- \\
1993\end{array}$ \\
\hline WCO2 & CCWE & incineration & $\begin{array}{l}\text { Diversified } \\
\text { Scientific } \\
\text { Services, } \\
\text { Kingston, TN }\end{array}$ & 12 & $\begin{array}{l}\text { None } \\
\text { (complete } \\
\text { destruc- } \\
\text { tion) }\end{array}$ & $\begin{array}{l}1991- \\
1993\end{array}$ \\
\hline
\end{tabular}


Table 2-6. Treatment of Land Disposal Restricted Waste for Disposal. (sheet 5 of 10)

\begin{tabular}{|c|c|c|c|c|c|c|}
\hline $\begin{array}{c}\text { Wastes } \\
\text { codes }\end{array}$ & $\begin{array}{l}\text { Required } \\
\text { treatment }\end{array}$ & $\begin{array}{l}\text { Planned } \\
\text { treatment }\end{array}$ & $\begin{array}{l}\text { Treatment } \\
\text { facility }\end{array}$ & $\begin{array}{l}\text { Facility } \\
\text { capacity } \\
\text { (m } / \text { day) }\end{array}$ & $\begin{array}{l}\text { Disposal } \\
\text { facility }\end{array}$ & $\begin{array}{l}\text { Treat- } \\
\text { ment } \\
\text { date }\end{array}$ \\
\hline \multicolumn{7}{|c|}{ 9. 183-H Solar Evaporation Basins Waste } \\
\hline U123 & incineration & TBD & TBD & TBD & TBD & TBD \\
\hline P030 & CCWE & TBD & WRAP & TBD & TBD & $1999^{f}$ \\
\hline P120 & stabilization & TBD & WRAP & TBD & TBD & $1999^{f}$ \\
\hline P029 & CCWE & TBD & WRAP & TBD & TBD & $1999^{f}$ \\
\hline P106 & CCWE & TBD & WRAP & TBD & TBD & $1999^{f}$ \\
\hline P098 & CCWE & TBD & WRAP & TBD & TBD & $1999^{f}$ \\
\hline 0007 & CCWE & TBD & WRAP & TBD & TBD & $1999^{f}$ \\
\hline WTOI & reduction & TBD & WRAP & TBD & TBD & $1999^{f}$ \\
\hline \multicolumn{7}{|c|}{ 10. PUREX Storage Tunnel 2 Waste (mercury) } \\
\hline D001 & deactivation & TBD & TBD & TBD & TBD & TBD \\
\hline D009 & amalgamation & TBD & TBD & TBD & TBD & TBD \\
\hline D011 & CCWE & TBD & TBD & TBD . & TBD & TBD \\
\hline WTO1 & reduction & TBD & TBD & TBD & TBD & TBD \\
\hline \multicolumn{7}{|c|}{ 11.a PUREX Storage Tunnels 1 and 2 Naste (lead) } \\
\hline D008 & $\begin{array}{l}\text { macro- } \\
\text { encapsulation }\end{array}$ & TBD & TBD & TBD & TBD & TBD \\
\hline WTOI & reduction & TBD & TBD & TBD & TBD & TBD \\
\hline \multicolumn{7}{|c|}{ 11.b PUREX Storage Tunnel 2 Waste (silver) } \\
\hline D001 & deactivation & TBD & TBD & TBD & TBD & TBD \\
\hline D011 & CCWE & TBD & TBD & TBD & TBD & TBD \\
\hline WTOI & reduction & TBD & TBD & TBD & TBD & TBD \\
\hline \multicolumn{7}{|c|}{ 12. PUREX Containment Building (lead and cadmium) } \\
\hline D006 & CCWE & TBD & TBD & TBD & TBD & TBD \\
\hline D008 & $\begin{array}{l}\text { macro- } \\
\text { encapsulation }\end{array}$ & TBD & TBD & TBD & TBD & TBD \\
\hline WTO1 & reduction & TBD & TBD & TBD & TBD & TBD \\
\hline
\end{tabular}


Table 2-6. Treatment of Land Disposal Restricted Waste for Disposal. (sheet 6 of 10)

\begin{tabular}{|c|c|c|c|c|c|c|}
\hline $\begin{array}{l}\text { Wastes } \\
\text { codes }\end{array}$ & $\begin{array}{l}\text { Required } \\
\text { treatment }\end{array}$ & $\begin{array}{l}\text { Planned } \\
\text { treatment }\end{array}$ & $\begin{array}{l}\text { Treatment } \\
\text { factlity }\end{array}$ & $\begin{array}{l}\text { Facility } \\
\text { capacity } \\
\left(\mathrm{m}^{3} / \text { day }\right)\end{array}$ & $\begin{array}{l}\text { Disposal } \\
\text { facility }\end{array}$ & $\begin{array}{l}\text { Treat- } \\
\text { ment } \\
\text { date }\end{array}$ \\
\hline \multicolumn{7}{|c|}{$\begin{array}{l}\text { 13. CWC Stored Low-Level, Transuranic, and PCB Wastes } \\
\text { Low-level waste }\end{array}$} \\
\hline F001 & $\mathrm{CCH}$ & Incineration & TBD & TBD & TBD & $1999^{f}$ \\
\hline F002 & $\mathrm{CCH}$ & Incineration & $T B D$ & TBD & TBD & $1999^{f}$ \\
\hline F003 & $\mathrm{CCH}$ & Incineration & TBD & TBD & TBD & $1999^{f}$ \\
\hline F004 & $\mathrm{CCW}$ & Incineration & TBD & TBD & TBD & $1999^{f}$ \\
\hline F005 & $\mathrm{CCW}$ & Incineration & TBD & TBD & TBD & $1999^{f}$ \\
\hline$D 001$ & deactivation & TBD & WRAP & TBD & TBD & $1999^{f}$ \\
\hline D002 & deactivation & TBD & WRAP & TBD & TBD & $1999^{f}$ \\
\hline D003 & deactivation & TBD & WRAP & TBD & TBD & $1999^{f}$ \\
\hline D004 & CCWE & TBD & WRAP & TBD & TBD & $1999^{f}$ \\
\hline D005 & CCWE & TBD & WRAP & TBD & TBD & $1999^{f}$ \\
\hline D006 & CCWE & TBD & WRAP & TBD & TBD & $1999^{f}$ \\
\hline 0007 & CCWE & TBD & WRAP & TBD & TBD & $1999^{f}$ \\
\hline D008 & $\begin{array}{l}\text { macro- } \\
\text { encapsulation }\end{array}$ & TBD & WRAP & TBD & TBD & $1999^{f}$ \\
\hline D009 & amalgamation & TBD & WRAP & TBD & TBD & $1999^{f}$ \\
\hline D010 & CCWE & TBD & WRAP & TBD & TBD & $1999^{f}$ \\
\hline D011 & CCWE & TBD & WRAP & TBD & TBD & $1999^{f}$ \\
\hline D012 & CCWE & TBD & WRAP & TBD & TBD & $1999^{f}$ \\
\hline D016 & CCWE & TBD & WRAP & TBD & TBD & $1999^{f}$ \\
\hline WTOI & reduction & TBD & WRAP & TBD & TBD & $1999^{f}$ \\
\hline WTO2 & none & TBD & WRAP & TBD & TBD & $1999^{f}$ \\
\hline WCO2 & none & TBD & WRAP & TBD & TBD & $1999^{f}$ \\
\hline WPO1 & reduction & TBD & WRAP & TBD & TBD & $1999^{f}$ \\
\hline WPO2 & none & TBD & WRAP & TBD & TBD & $1999^{f}$ \\
\hline U080 & CCWE & TBD & WRAP & TBD & TBD & $1999^{f}$ \\
\hline U123 & incineration & TBD & TBD & TBD & TBD & TBD \\
\hline 0161 & CCWE & TBD & WRAP & TBD & TBD & $1999^{f}$ \\
\hline
\end{tabular}


Table 2-6. Treatment of Land Disposal Restricted Waste for Disposal. (sheet 7 of 10)

\begin{tabular}{|c|c|c|c|c|c|c|}
\hline $\begin{array}{l}\text { Wastes } \\
\text { codes }\end{array}$ & $\begin{array}{l}\text { Required } \\
\text { treatment }\end{array}$ & $\begin{array}{l}\text { Planned } \\
\text { treatment }\end{array}$ & $\begin{array}{l}\text { Treatment } \\
\text { facility }\end{array}$ & $\begin{array}{l}\text { Facility } \\
\text { capacity } \\
\text { (mday) }\end{array}$ & $\begin{array}{l}\text { Disposal } \\
\text { facility }\end{array}$ & $\begin{array}{l}\text { Treat- } \\
\text { ment } \\
\text { date }\end{array}$ \\
\hline P029 & CCWE & TBD & WRAP & TBD & TBD & $1999^{f}$ \\
\hline P030 & CCWE & TBD & WRAP & TBD & TBD & $1999^{f}$ \\
\hline P098 & CCWE & TBD & WRAP & TBD & TBD & $1999^{f}$ \\
\hline P106 & CCWE & TBD & WRAP & TBD & TBD & $1999^{f}$ \\
\hline P120 & stabilization & TBD & WRAP & TBD & TBD & $1999^{f}$ \\
\hline PCBs & incineration & TBD & TBD & TBD & TBD & TBD \\
\hline \multicolumn{7}{|c|}{ Transuranic waste } \\
\hline F003 & None & TBD & WRAP & TBD & WIPP & $1999^{f}$ \\
\hline F005 & None? & TBD & WRAP & TBD & WIPP & $1999^{f}$ \\
\hline Do01 & None & TBD & WRAP & TBD & WIPP & $1999^{f}$ \\
\hline 0002 & None" & TBD & WRAP & TBD & WIPP & $1999^{f}$ \\
\hline D006 & None & TBD & WRAP & TBD & WIPP & $1999^{f}$ \\
\hline 0007 & None & TBD & WRAP & TBD & WIPP & $1999^{f}$ \\
\hline D008 & None & TBD & WRAP & TBD & WIPP & $1999^{f}$ \\
\hline D009 & None & TBD & WRAP & TBD & WIPP & $1999^{f}$ \\
\hline WTO1 & None & TBD & WRAP & TBD & WIPP & $1999^{f}$ \\
\hline WTO2 & None & TBD & WRAP & TBD & WIPP & $1999^{f}$ \\
\hline WCO2 & None? & TBD & WRAP & TBD & WIPP & $1999^{f}$ \\
\hline PCBs & None & TBD & TBD & TBD & WIPP & TBD \\
\hline \multicolumn{7}{|c|}{$\begin{array}{l}\text { 14. Retrievably Stored Low-Level and Transuranic Wastes } \\
\text { Low-level waste }\end{array}$} \\
\hline F001 & $\mathrm{CCH}$ & TBD & WRAP & TBD & TBD & $1999^{f}$ \\
\hline F003 & $\mathrm{CCH}$ & TBD & WRAP & TBD & TBD & $1999^{f}$ \\
\hline F005 & $\mathrm{CCH}$ & TBD & WRAP & TBD & TBD & $1999^{f}$ \\
\hline 0001 & deactivation & TBD & WRAP & TBD & TBD & $1999^{f}$ \\
\hline 0003 & deactivation & TBD & WRAP & TBD & TBD & $1999^{f}$ \\
\hline 0005 & CCWE & TBD & WRAP & TBD & TBD & $1999^{7}$ \\
\hline D006 & CCWE & TBD & WRAP & TBD & TBD & $1999^{f}$ \\
\hline D007 & CCWE & TBD & WRAP & TBD & TBD & $1999^{f}$ \\
\hline
\end{tabular}


Table 2-6. Treatment of Land Disposal Restricted Waste for Disposal. (sheet 8 of 10)

\begin{tabular}{|c|c|c|c|c|c|c|}
\hline $\begin{array}{l}\text { Wastes } \\
\text { codes }\end{array}$ & $\begin{array}{l}\text { Required } \\
\text { treatment }\end{array}$ & $\begin{array}{l}\text { Planned } \\
\text { treatment }\end{array}$ & $\begin{array}{l}\text { Treatment } \\
\text { facility }\end{array}$ & $\begin{array}{l}\text { Facility } \\
\text { capacity } \\
\left(m^{3} / \text { day }\right)\end{array}$ & $\begin{array}{l}\text { Disposal } \\
\text { facility }\end{array}$ & $\begin{array}{l}\text { Treat- } \\
\text { ment } \\
\text { date }\end{array}$ \\
\hline 0008 & $\begin{array}{l}\text { macro- } \\
\text { encapsulation }\end{array}$ & TBD & WRAP & TBD & TBD & $1999^{f}$ \\
\hline 0009 & amalgamation & TBD & WRAP & TBD & TBD & $1999^{f}$ \\
\hline$\overline{0011}$ & CCWE & TBD & WRAP & TBD & TBD & $1999^{f}$ \\
\hline WTO1 & reduction & TBD & WRAP & TBD & TBD & $1999^{f}$ \\
\hline WT02 & none & TBD & WRAP & TBD & TBD & $1999^{f}$ \\
\hline WCO2 & none & TBD & WRAP & TBD & TBD & $1999^{f}$ \\
\hline WPOI & reduction & TBD & WRAP & TBD & TBD & $1999^{f}$ \\
\hline PCBS & incineration & TBD & TBD & TBD & TBD & TBD \\
\hline \multicolumn{7}{|c|}{ Transuranic waste } \\
\hline 0006 & None ${ }^{9}$ & TBD & WRAP & TBD & WIPP & $1999^{\prime}$ \\
\hline 0008 & None? & TBD & WRAP & TBD & WIPP & $1999^{f}$ \\
\hline WTOI & None" & TBD & WRAP & TBD & WIPP & $1999^{4}$ \\
\hline P015 & None" & TBD & WRAP & TBD & WIPP & $1999^{f}$ \\
\hline \multicolumn{7}{|c|}{ 15. TRUSAF Stored Waste } \\
\hline 0002 & none ${ }^{9}$ & none & -- & -- & WIPP & -- \\
\hline D005 & none ${ }^{8}$ & none & -- & -- & WIPP & -- \\
\hline D006 & none & none & -- & -- & WIPP & -- \\
\hline 0007 & none & none & -- & -- & WIPP & -- \\
\hline D008 & none $e^{9}$ & none & $-\infty$ & -- & WIPP & -- \\
\hline 0009 & none & none & -- & -- & WIPP & -- \\
\hline WCO2 & none & none & -- & -- & WIPP & -- \\
\hline WPO1 & none & none & -- & -- & WIPP & $-\infty$ \\
\hline WTO1 & none & none & -- & -- & WIPP & -- \\
\hline \multicolumn{7}{|c|}{ 16. $303-K$ Stored Waste } \\
\hline F001 & $\mathrm{CCH}$ & TBD & WRAP & TBD & TBD & $1999^{f .1}$ \\
\hline F002 & $\mathrm{CCH}$ & TBD & WRAP & TBD & TBD & $1999^{f .1}$ \\
\hline D001 & deactivation & TBD & WRAP & TBD & TBD & $1999^{f}$ \\
\hline D006 & CCWE & TBD & WRAP & TBD & TBD & $1999^{f}$ \\
\hline
\end{tabular}


Table 2-6. Treatment of Land Disposal Restricted Waste for Disposal. (sheet 9 of 10)

\begin{tabular}{|l|l|l|l|l|l|l|}
\hline $\begin{array}{c}\text { Wastes } \\
\text { codes }\end{array}$ & $\begin{array}{c}\text { Required } \\
\text { treatment }\end{array}$ & $\begin{array}{c}\text { P1 anned } \\
\text { treatment }\end{array}$ & $\begin{array}{c}\text { Treatment } \\
\text { facility }\end{array}$ & $\begin{array}{c}\text { Facility } \\
\text { capacity } \\
\text { (m/day) }\end{array}$ & $\begin{array}{c}\text { Disposal } \\
\text { facility }\end{array}$ & $\begin{array}{c}\text { Treat- } \\
\text { ment } \\
\text { date }\end{array}$ \\
\hline D007 & CCWE & TBD & WRAP & TBD & TBD & $1999^{\dagger}$ \\
\hline D008 & $\begin{array}{l}\text { macro- } \\
\text { encapsulation }\end{array}$ & TBD & WRAP & TBD & TBD & $1999^{+}$ \\
\hline D039 & $\begin{array}{l}\text { none } \\
\text { currently } \\
\text { promulgated }\end{array}$ & TBD & WRAP & TBD & TBD & $1999^{\dagger}$ \\
\hline WT01 & none & TBD & WRAP & TBD & TBD & $1999^{\dagger}$ \\
\hline WCO2 & reduction & TBD & WRAP & TBD & TBD & $1999^{\dagger}$ \\
\hline WP01 & reduction & TBD & WRAP & TBD & TBD & $1999^{f}$ \\
\hline
\end{tabular}

Treatment requitred by 40 CFR 268. Nonwastewater category assumed for this table. Deactivation treatment standards (e.g. D001 waste) were affected by the May 24, 1993 emergency rule. These wastes must al so be treated to meet F039 concentration standards.

The new Tri-Party Agreement strategy calls for pretreatment of essentially all wastes within DSTs and SSTs, the resulting streams being processed through either a HLW or LLW vitrification facility. Therefore, the individual streams such as NCAW and NCRW have been combined to simplify the table.

'Vitrification factlities for both the LLW and HLW fractions resulting from pretreatment have yet to be designed.

Treatment capacity for the LLW and HLW facilities have not been established. The LLW facility, however, will have a minimum capacity of 100 metric tons per day. Wastes wili be retrieved from the SSTs to the extent needed for closure. 'The facility for treating this waste is available on this date. This waste will be treated based on facility operating schedules. For the WRAP, dates given are for Module 2 (preceding submodulization to modules 2A and 2B). However, some wastes may be able to be treated sooner (1996) in Module 1. If the change request for WRAP Facility, Module 2A, is approved, some activities will occur after 1999. The assumption is made that no treatment is required as WIPP is expected to operate under a no-migration petition.

"Only a partial list of waste codes is given (see note for this stream in Table 2-3).

These degreaser solvent wastes are to be sent off site for treatment. 
Table 2-6. Treatment of Land Disposal Restricted Waste for Disposal. (sheet 10 of 10)

CCW - Constituent concentrations in waste.

CWC - Central Waste Complex.

CCWE = Constituent concentrations in waste extract.

DST = Double-shell tank.

ETF - Effluent Treatment Factlity.

LLBG - Low-level burial grounds.

LLW = Low-level waste.

LWVP - LoW-level waste vitrification plant.

PCB - Polychlorinated biphenyl.

PUREX - Plutonium-Uranium Extraction (Facility).

SALDS - State-approved land disposal structure.

$T B D=$ To be determined.

TRUSAF = Transuranic Waste Storage and Assay Facility.

WIPP - Waste Isolation Pllot Piant.

WRAP - Waste Receiving and Processing (Facillty). 
Table 2-7. Waste Reduction Activities for Hanford Site Land Disposal Mixed Wastes. (sheet 1 of 2)

\begin{tabular}{|c|c|c|c|}
\hline Waste & Method to reduce & $\begin{array}{l}\text { Schedule for } \\
\text { implementing } \\
\text { waste reduction } \\
\text { procedures }\end{array}$ & $\begin{array}{l}\text { Projected } \\
\text { waste } \\
\text { reduction }\end{array}$ \\
\hline 1. DST Waste" & $\begin{array}{l}\text { - Evaporation } \\
\text { - Minimize frequency of } \\
\text { flush } \\
\text { - Minimize flush volumes }\end{array}$ & under way & $80 \%$ \\
\hline $\begin{array}{l}\text { 2. PUREX Aging } \\
\text { Waste }\end{array}$ & $\begin{array}{l}\text { - Optimum control of the } \\
\text { evaporator waste flow } \\
\text { concentration overfiow } \\
\text { rate } \\
\text { - Evaporation }\end{array}$ & $\begin{array}{l}\text { under way } \\
\text { (Aging waste will } \\
\text { no longer be } \\
\text { generated.) }\end{array}$ & $21 \%$ \\
\hline 3. SST Waste & $\begin{array}{l}\text { - Waste is no longer being } \\
\text { added to SSTs }\end{array}$ & NA & NA \\
\hline $\begin{array}{l}\text { 4. 242-A Evapor- } \\
\text { ator Process } \\
\text { Condensate }\end{array}$ & $\begin{array}{l}\text { - Effluent Treatment } \\
\text { Facility will remove } \\
\text { ammonia, aqueous, salts, } \\
\text { metal tons, and organics }\end{array}$ & 1995 & $>99 \%$ \\
\hline $\begin{array}{l}4843 \text { Sodium } \\
\text { Storage } \\
\text { Facilitty } \\
\text { Waste }\end{array}$ & $\begin{array}{l}\text { Deactivate sodium by } \\
\text { converting it to } \\
\text { carbonate (or other } \\
\text { treatment method) }\end{array}$ & TBD & $>99 \%$ \\
\hline $\begin{array}{l}\text { 6. PUREX Ammonia } \\
\text { Scrubber Waste }\end{array}$ & $N A^{c}$ & -- & -- \\
\hline $\begin{array}{l}\text { 7. PUREX Process } \\
\text { Condensate }\end{array}$ & $N A^{C}$ & -- & -- \\
\hline 8. Hexone Waste & - Distill and incinerate & $\begin{array}{l}\text { Distillation } \\
\text { complete (1990), } \\
\text { incineration to } \\
\text { be completed in } \\
1994\end{array}$ & $88 \%$ \\
\hline $\begin{array}{l}\text { 183-H Solar } \\
\text { Evaporatton } \\
\text { Basins Waste }\end{array}$ & - Evaporate 1tquid & Complete (1990) & unknown \\
\hline $\begin{array}{l}\text { 10. PUREX Storage } \\
\text { Tunnel } 2 \text { Waste } \\
\text { (mercury) }\end{array}$ & $\begin{array}{l}\text { - Segregation from } \\
\text { nonhazardous waste }\end{array}$ & ongoing & variable \\
\hline $\begin{array}{l}\text { 11. PUREX Storage } \\
\text { Tunnels } 1 \text { and } \\
2 \text { Waste (lead) } \\
\text { and Tunnel } 2 \\
\text { Waste (silver) }\end{array}$ & $\begin{array}{l}\text { Segregation from } \\
\text { nonhazardous waste }\end{array}$ & ongoing & variable \\
\hline
\end{tabular}


Table 2-7. Waste Reduction Activities for Hanford Site Land Disposal Mixed Wastes. (sheet 2 of 2)

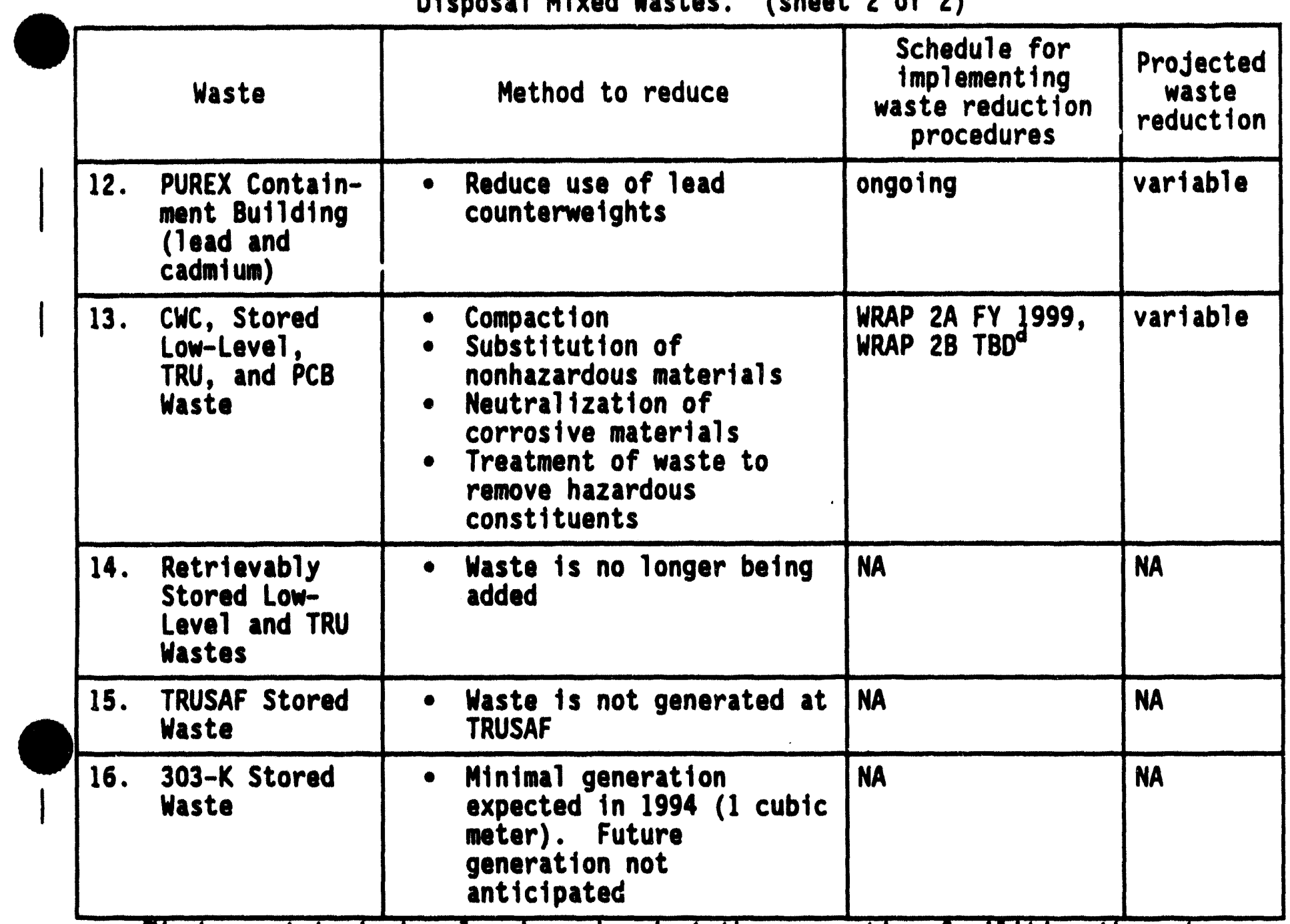

Waste sent to tanks also is reduced at the generating factitities through pretreatment (e.g., destroying ammonia) and recyciling of streams.

Waste sodium also is recycled at the generation point (Fast Flux Test Factlity).

'Anmonia Scrubber and Process Condensate will remain inactive; PUREX Plant has been officially notified to enter shutdown because of a September 24, 1992 Secretarial deciston to el iminate PUREX Operation as an option for processing N Reactor fuel.

Assumes that the WRAP Facility, Module 2, will be included in the M-33-00 change package.

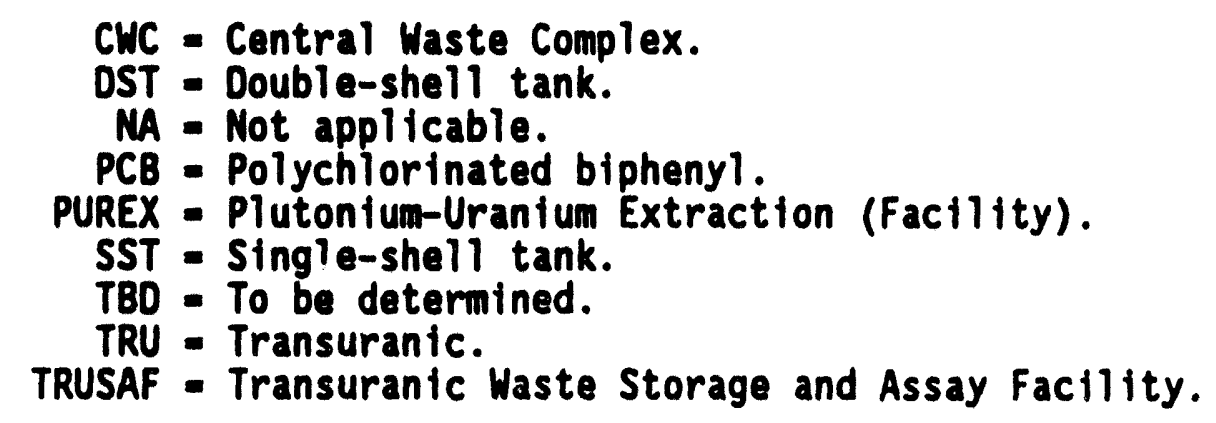


DOE/RL-94-21

This page intentionally left blank.

T2-7.3 


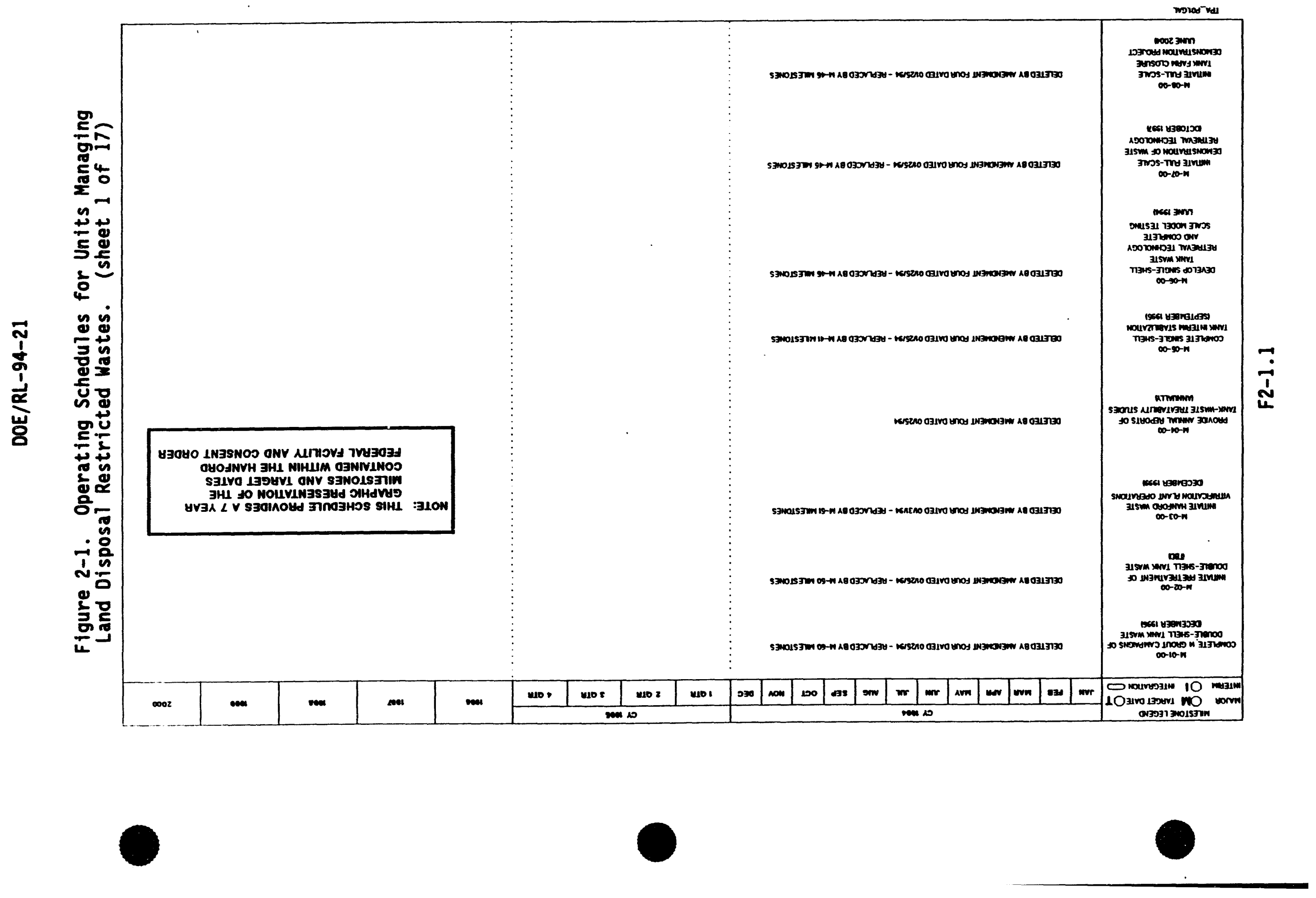



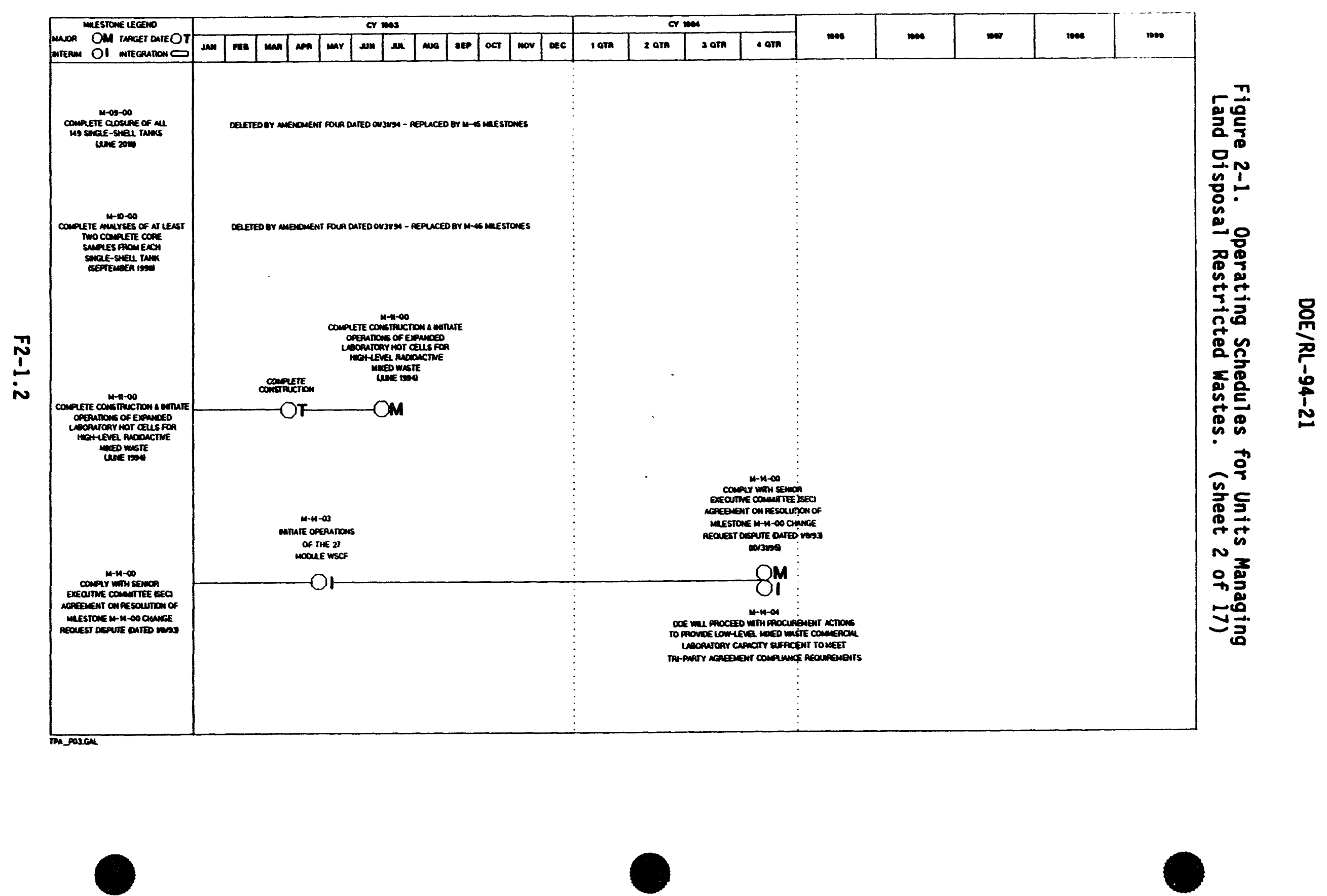


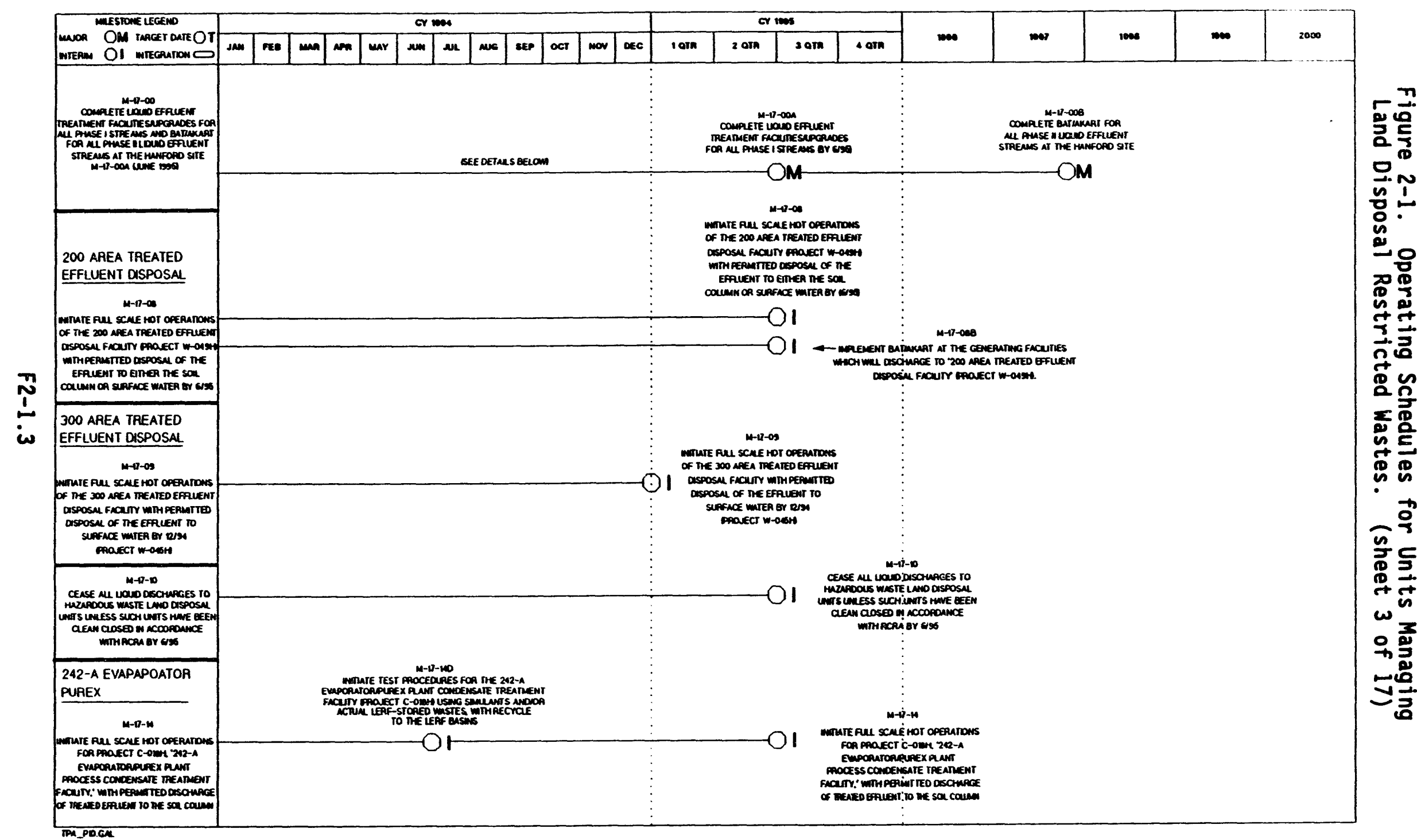




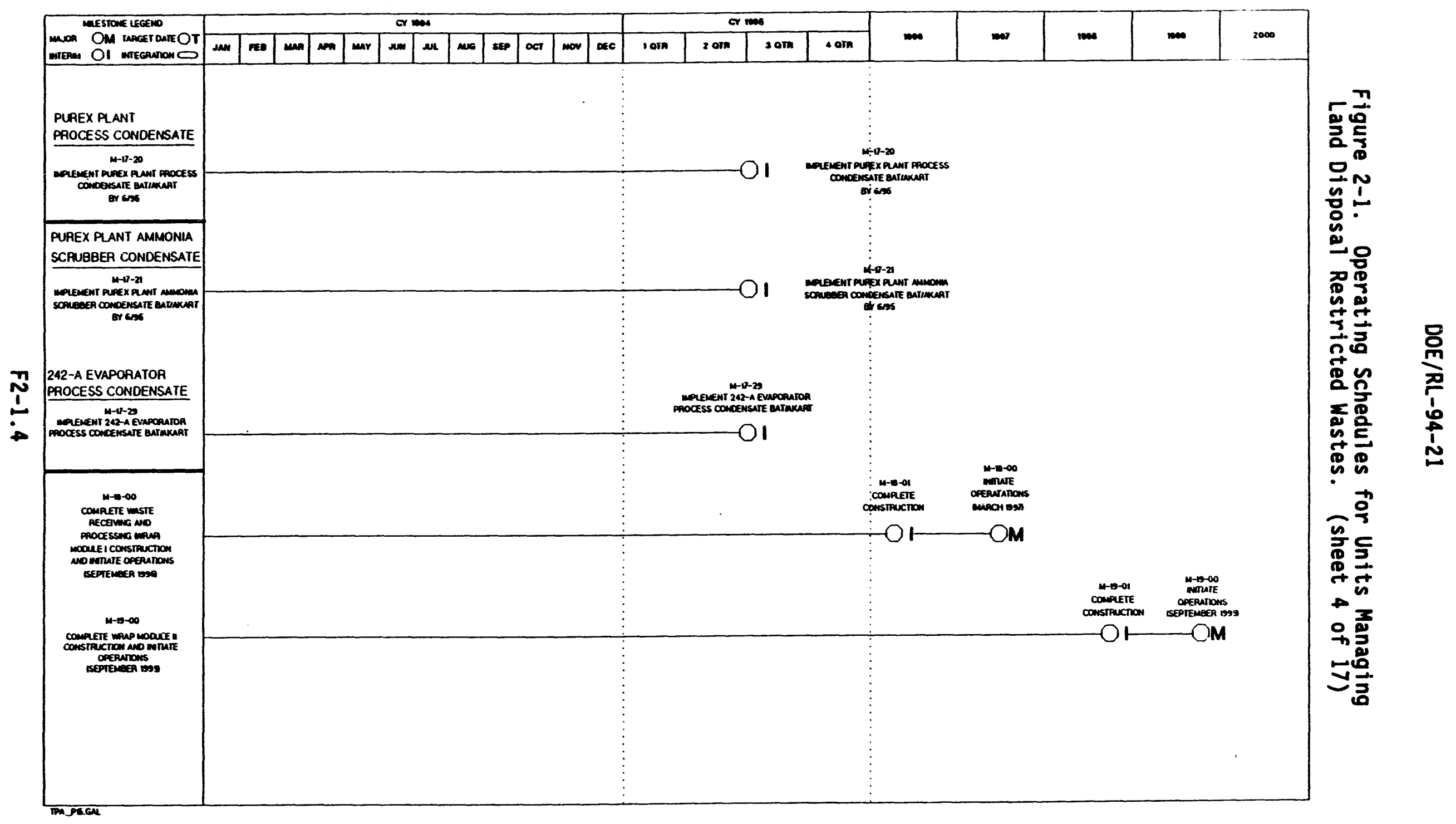




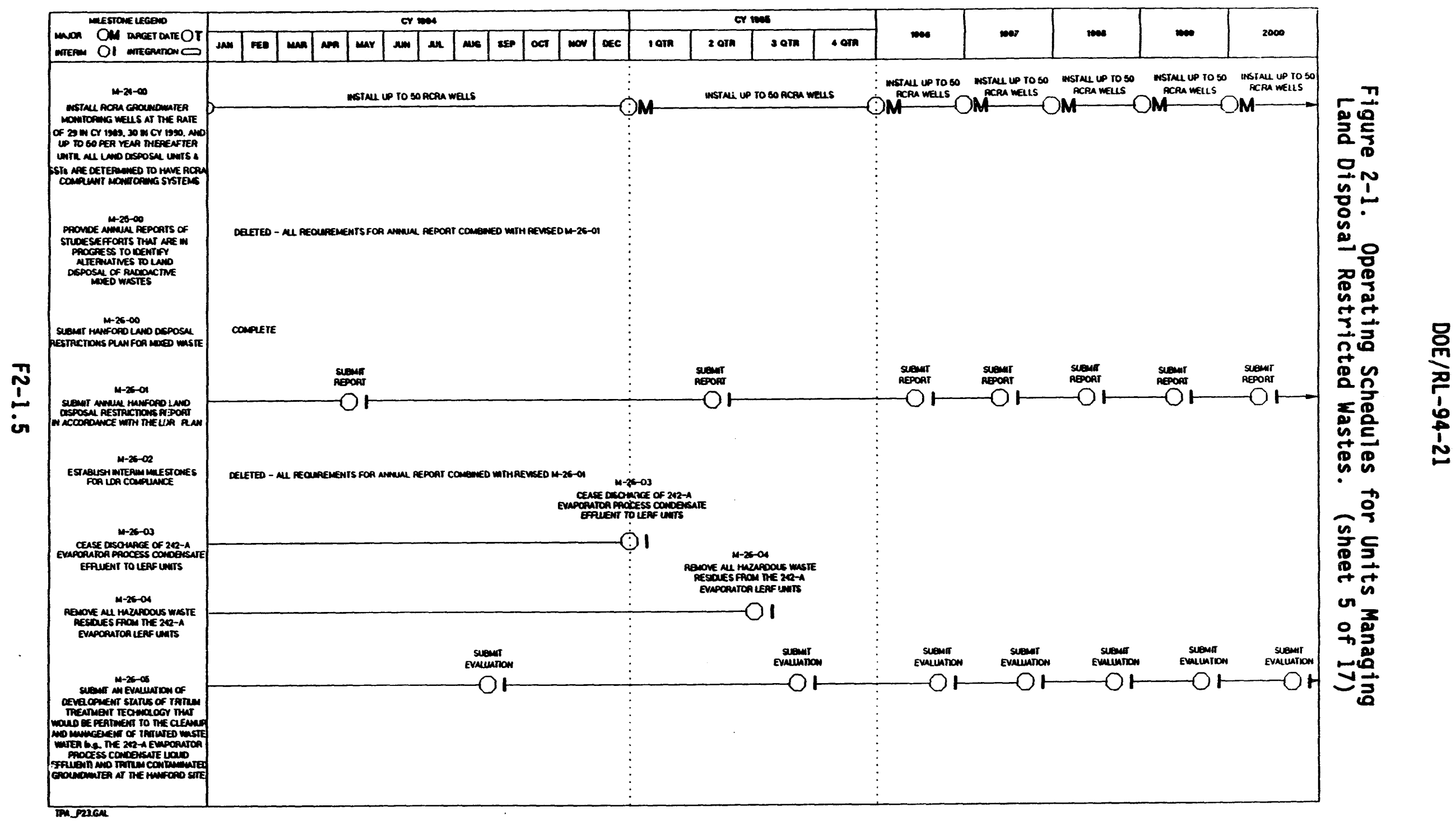




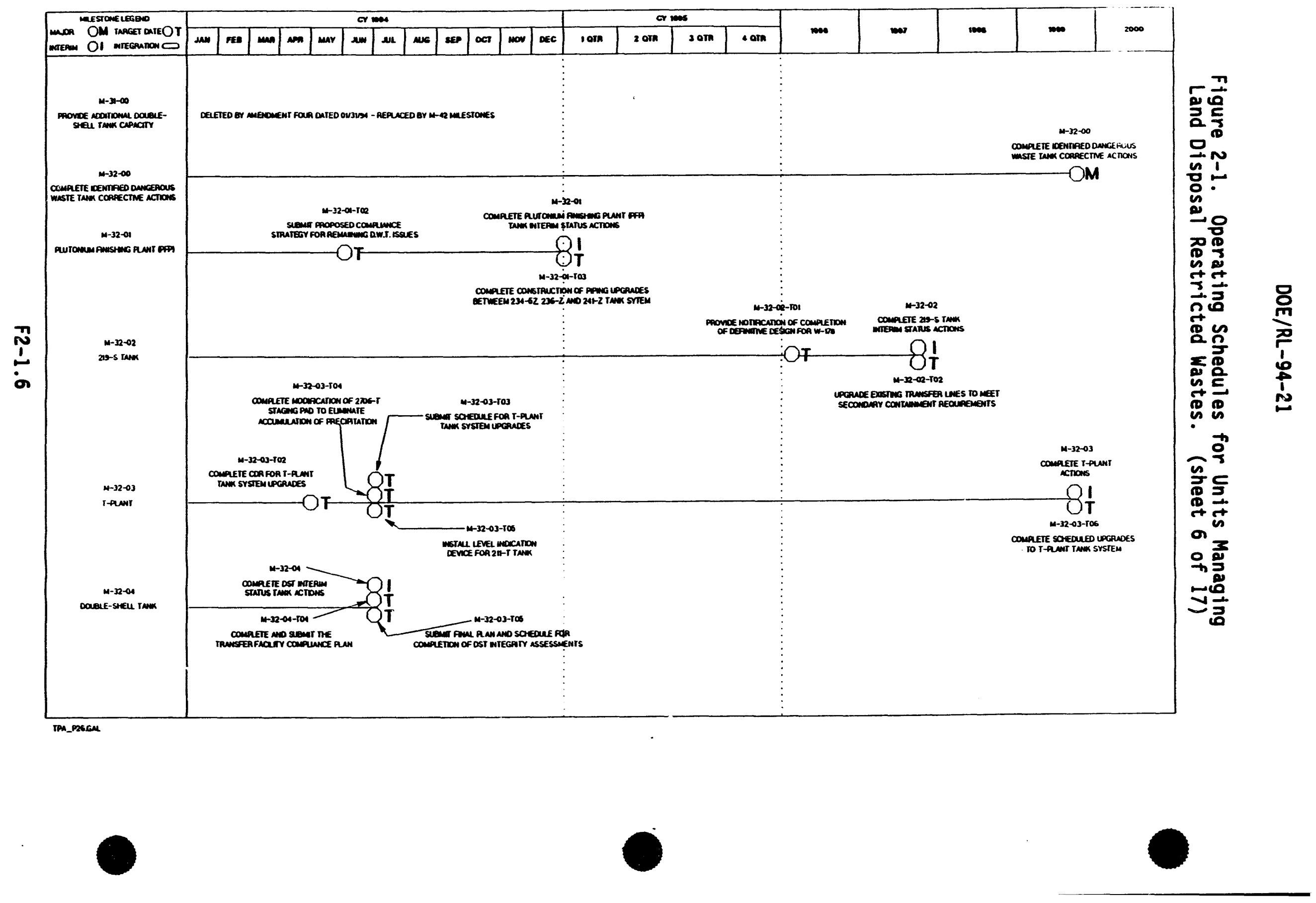


Figure 2-1. Operating Schedules for Units Managing Land Disposal Restricted Wastes. (sheet 7 of 17)

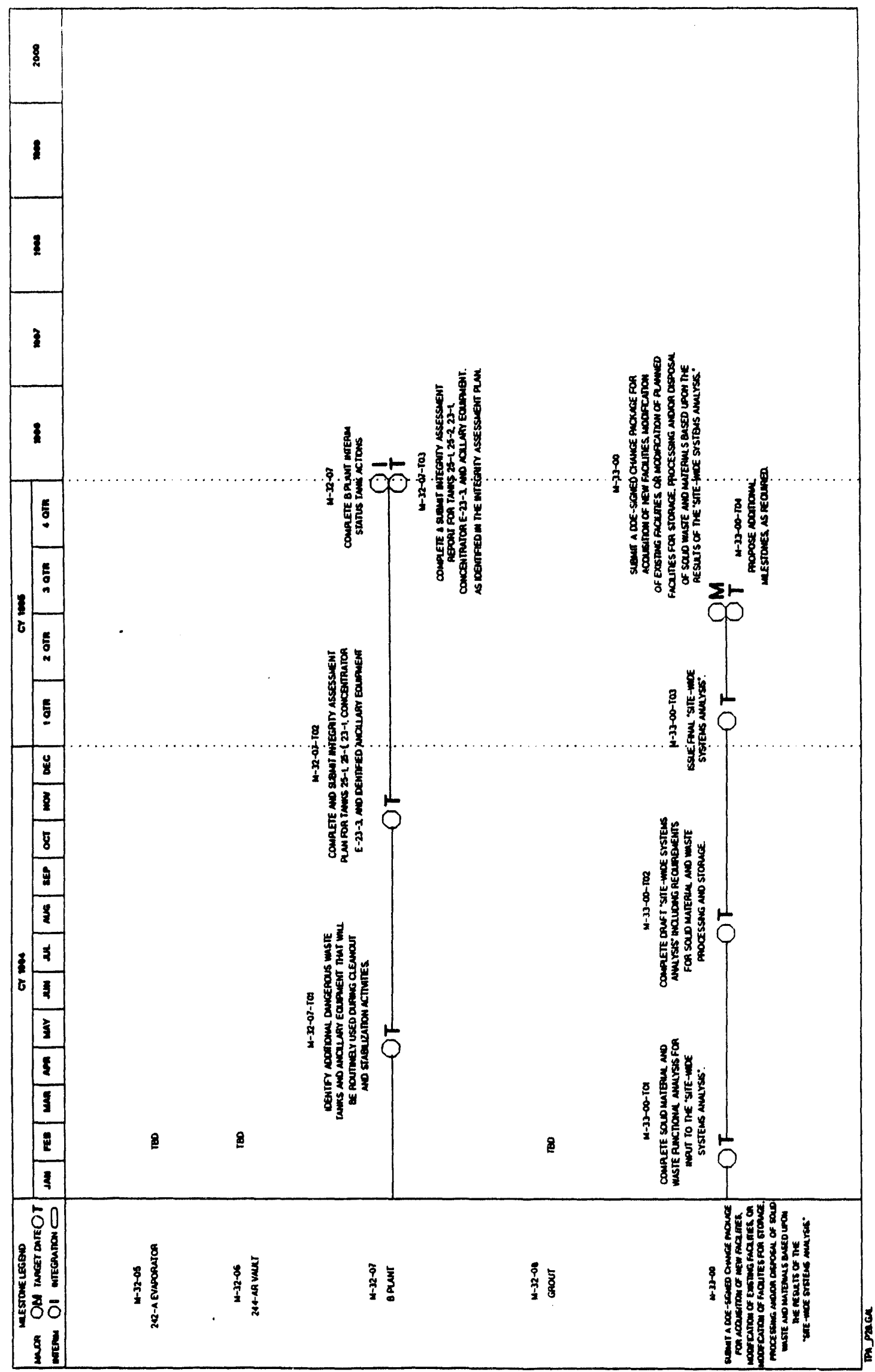

F2-1.7 


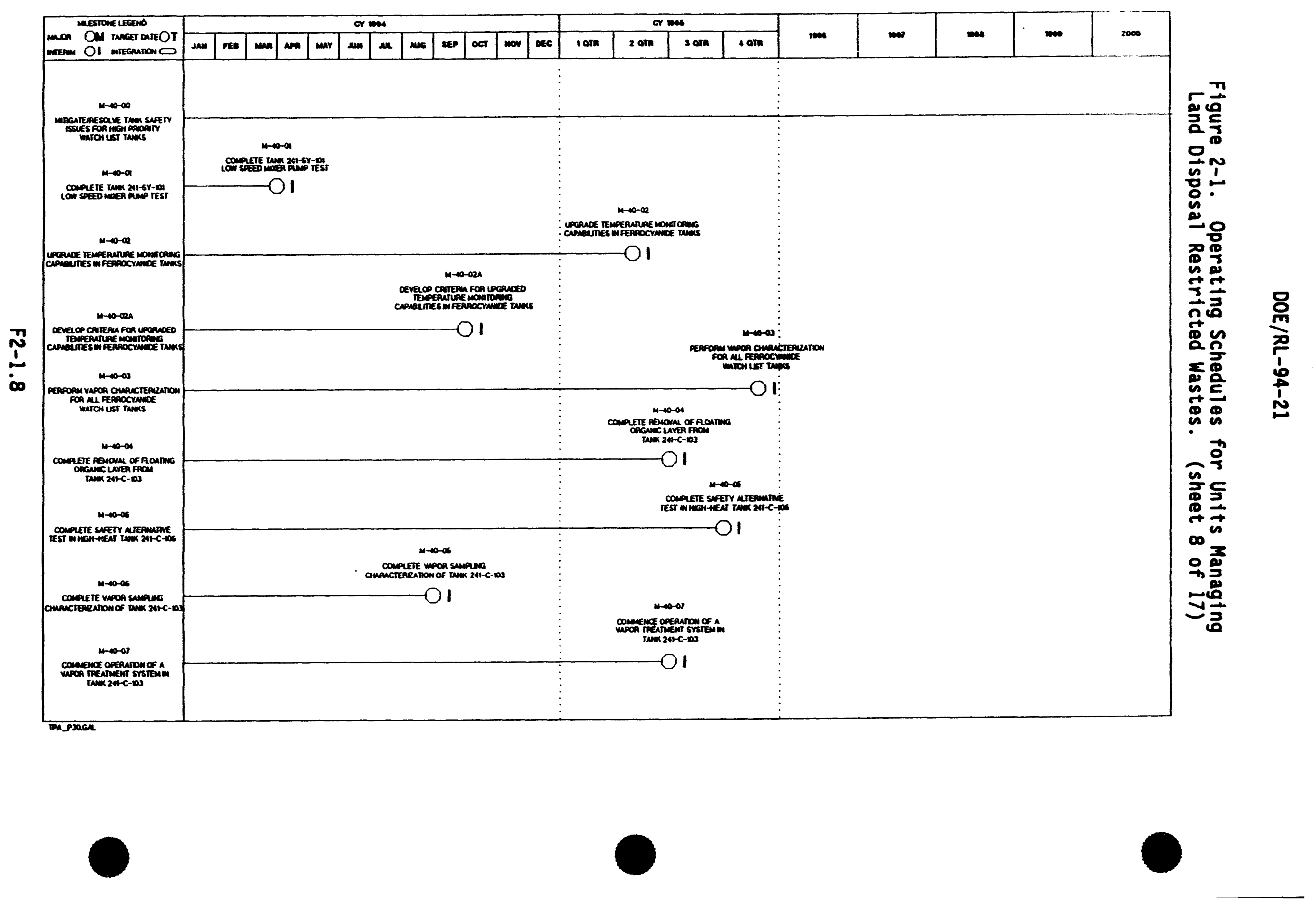


Figure 2-1. Operating Schedules for Units Managing
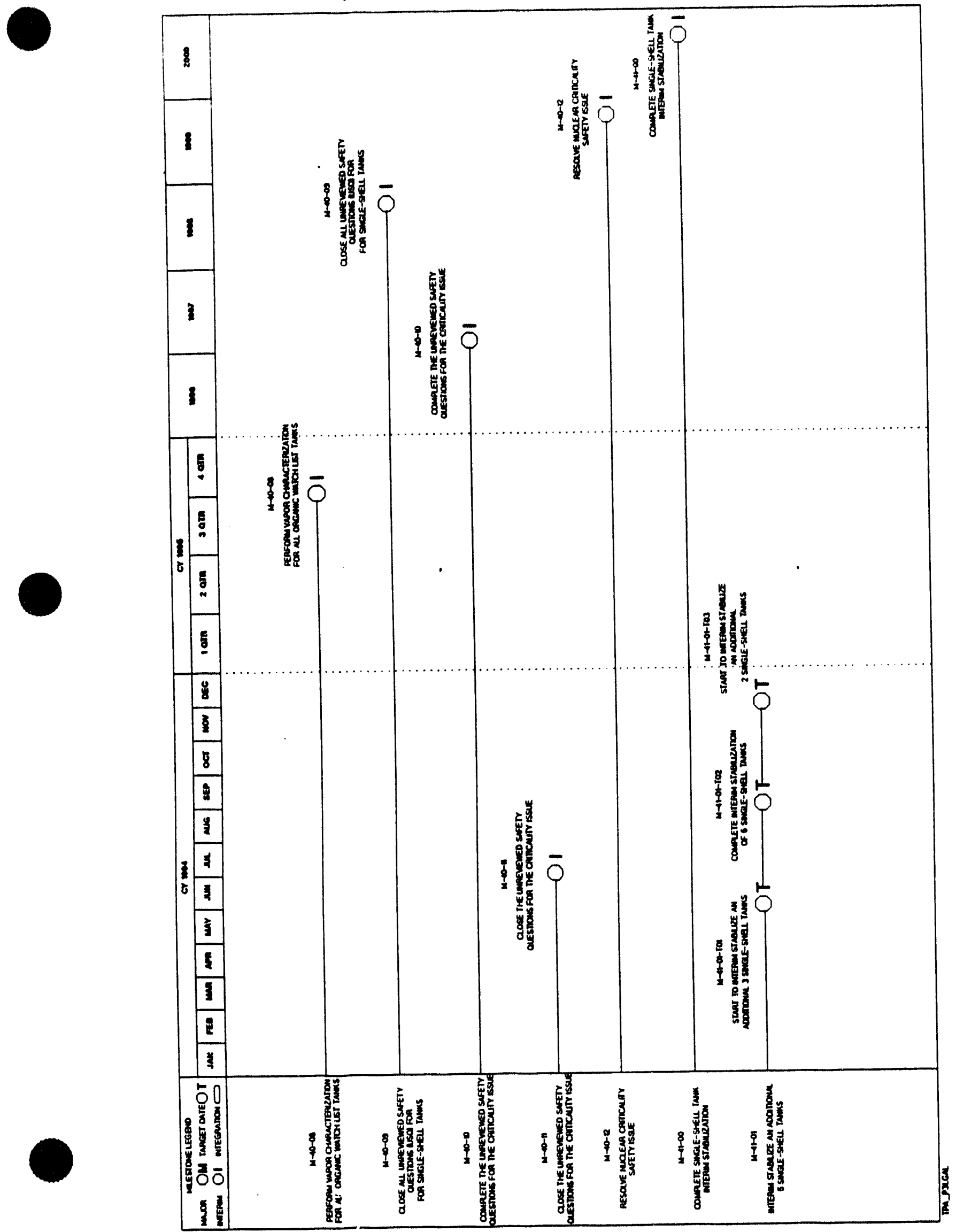

F2-1.9 
Figure 2-1. Operating Schedules for Units Managing Land Disposal Restricted Wastes. (sheet 10 of 17)

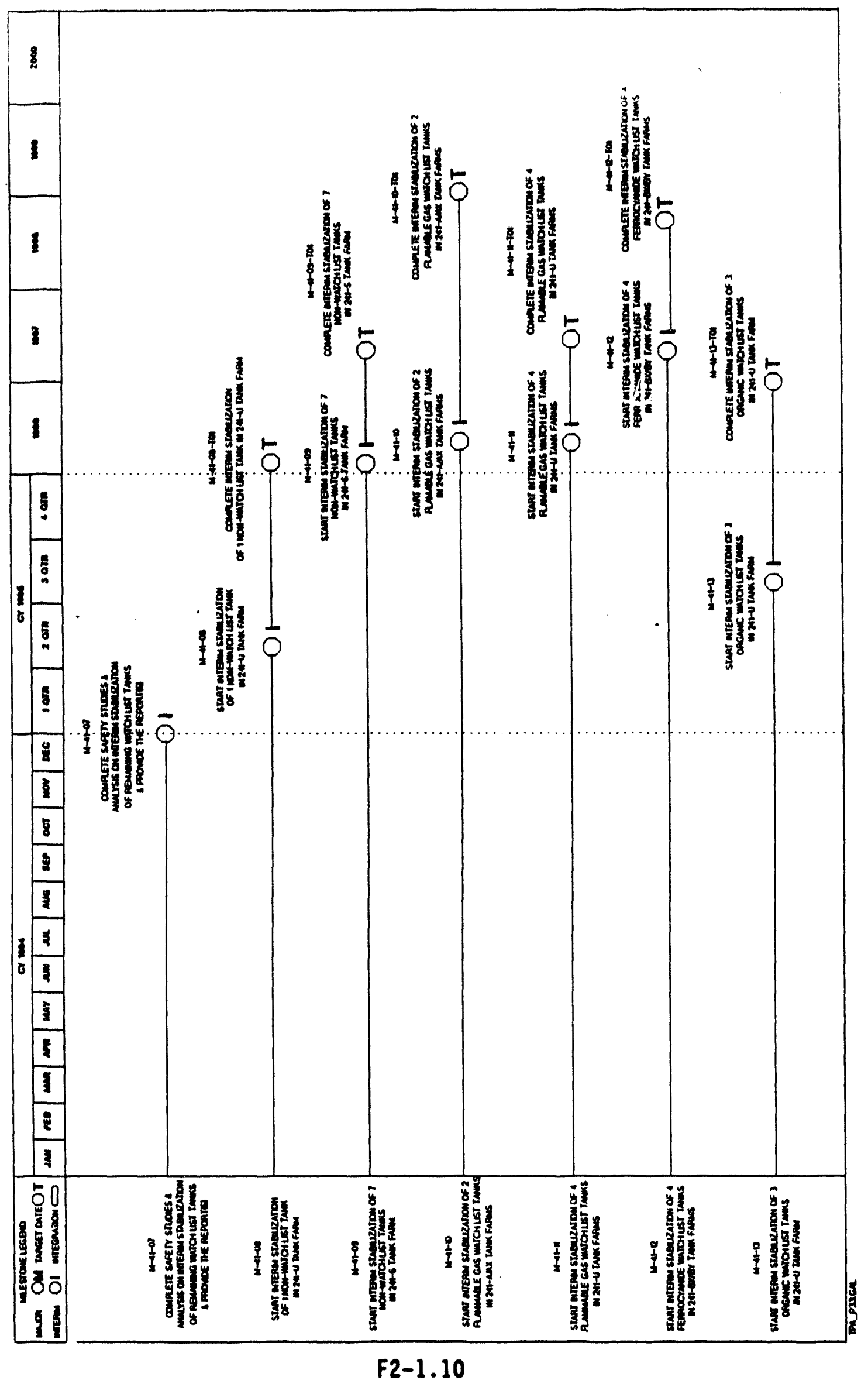




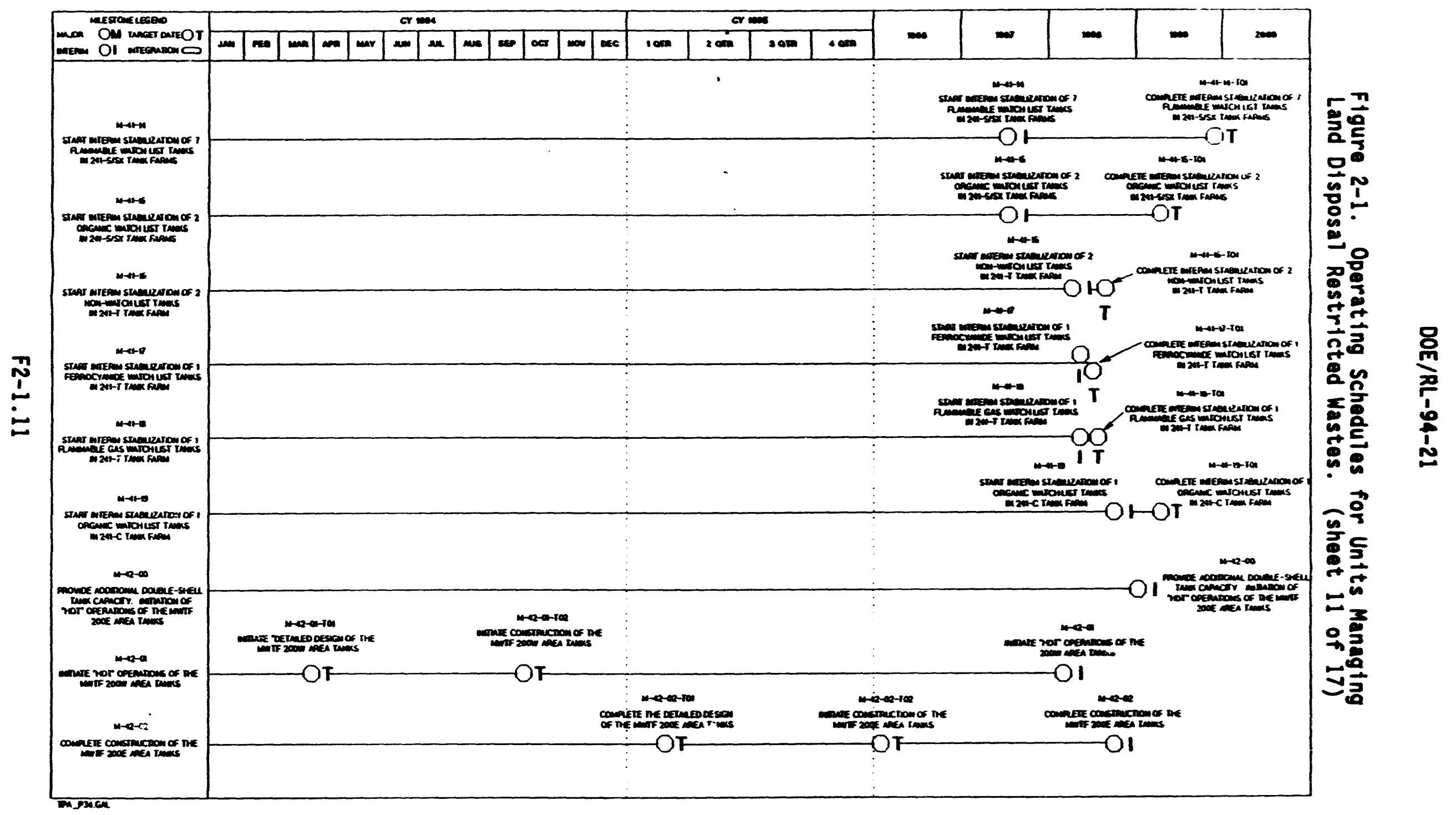


Figure 2-1. Operating Schedules for Units Managing Land Disposal Restricted Wastes. (sheet 12 of 17)

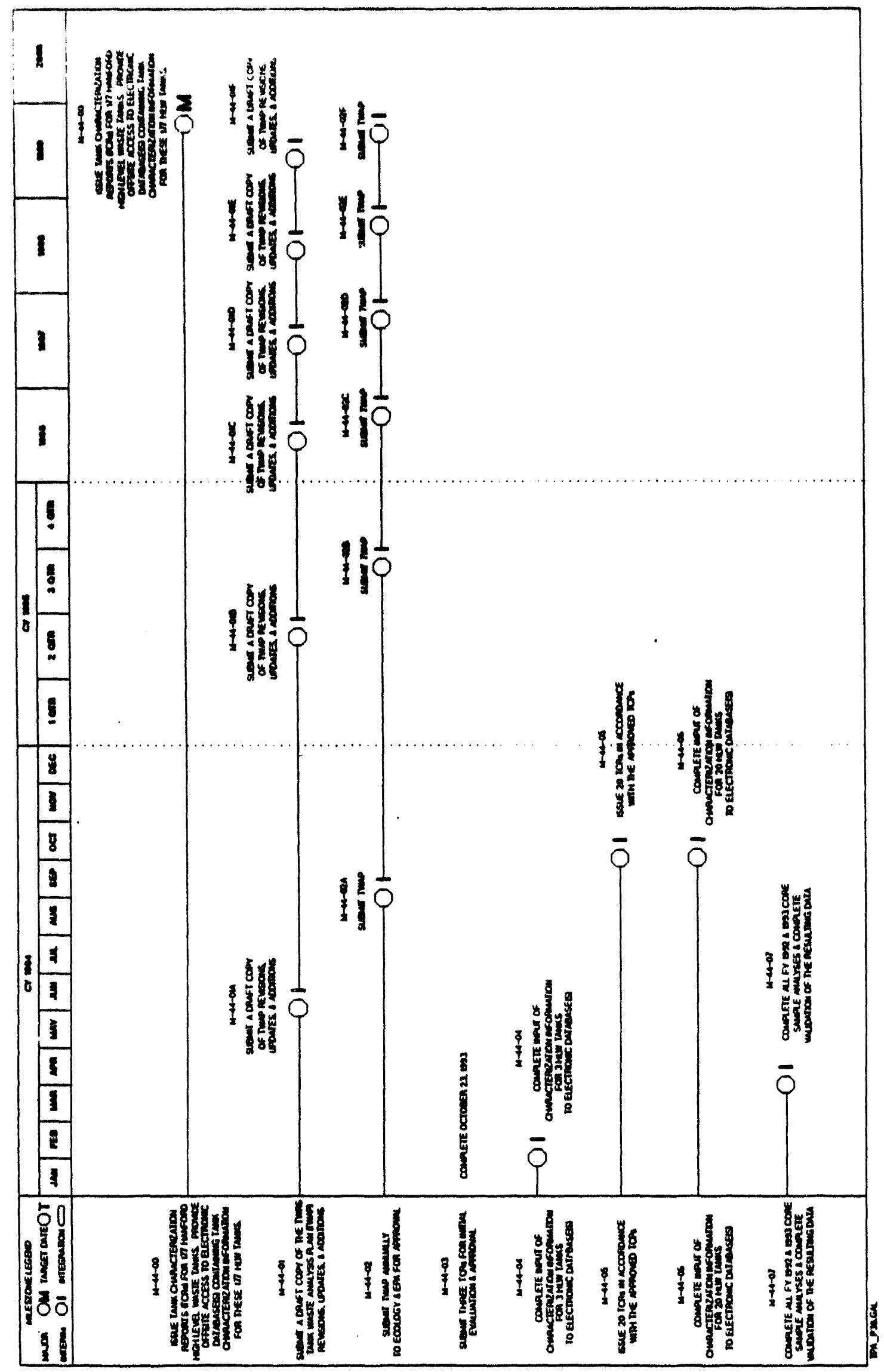

F2-1.12 


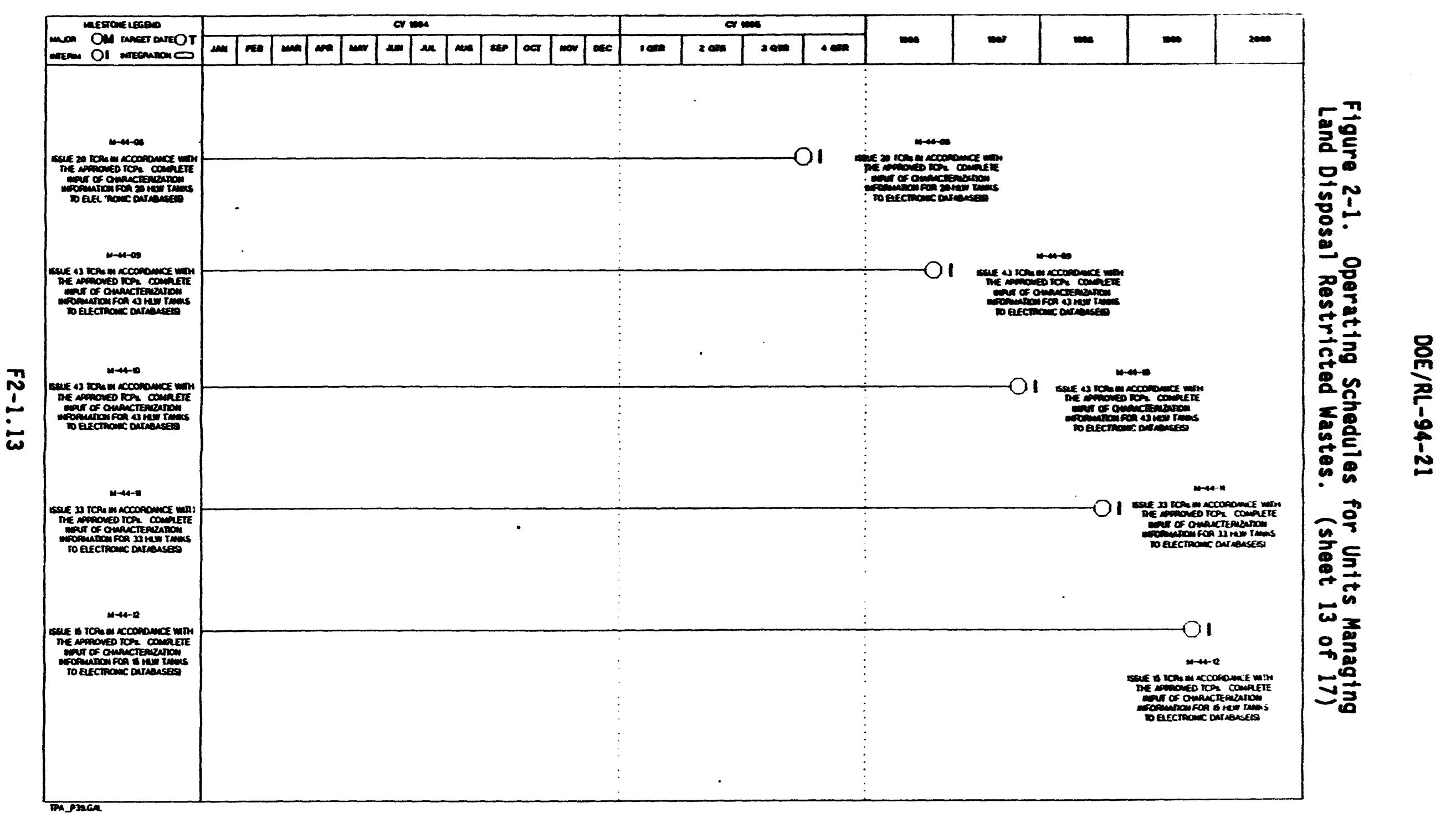




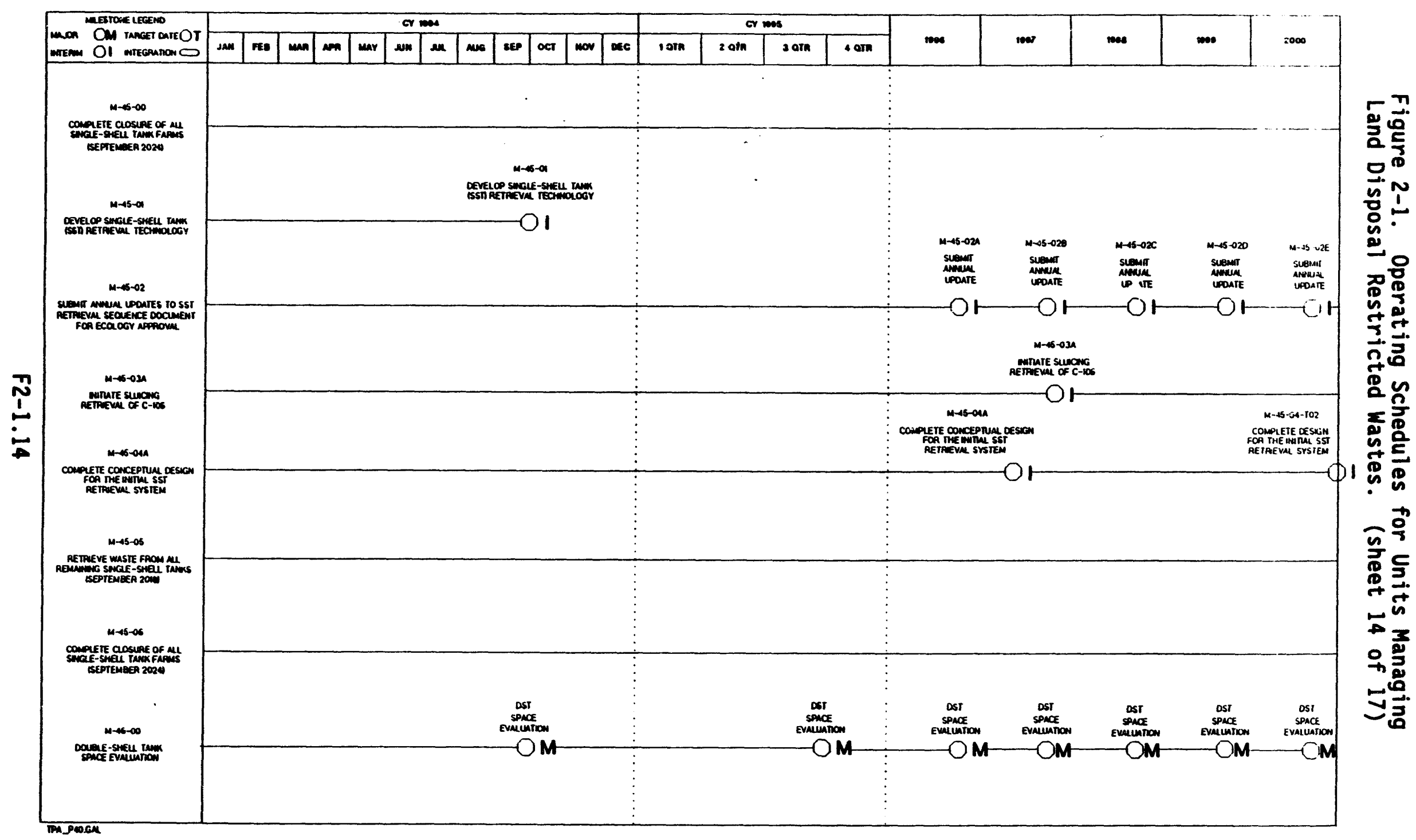




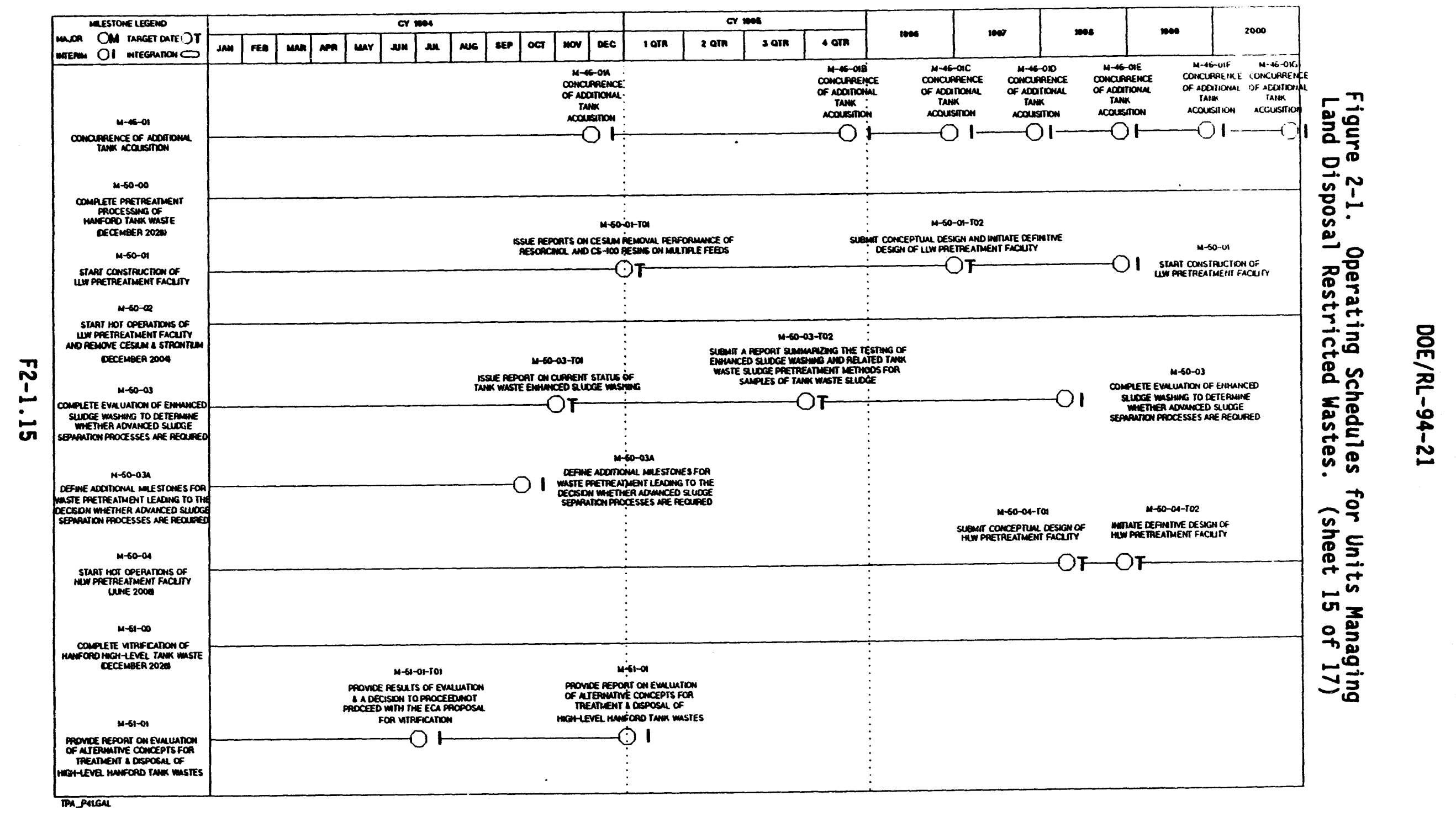




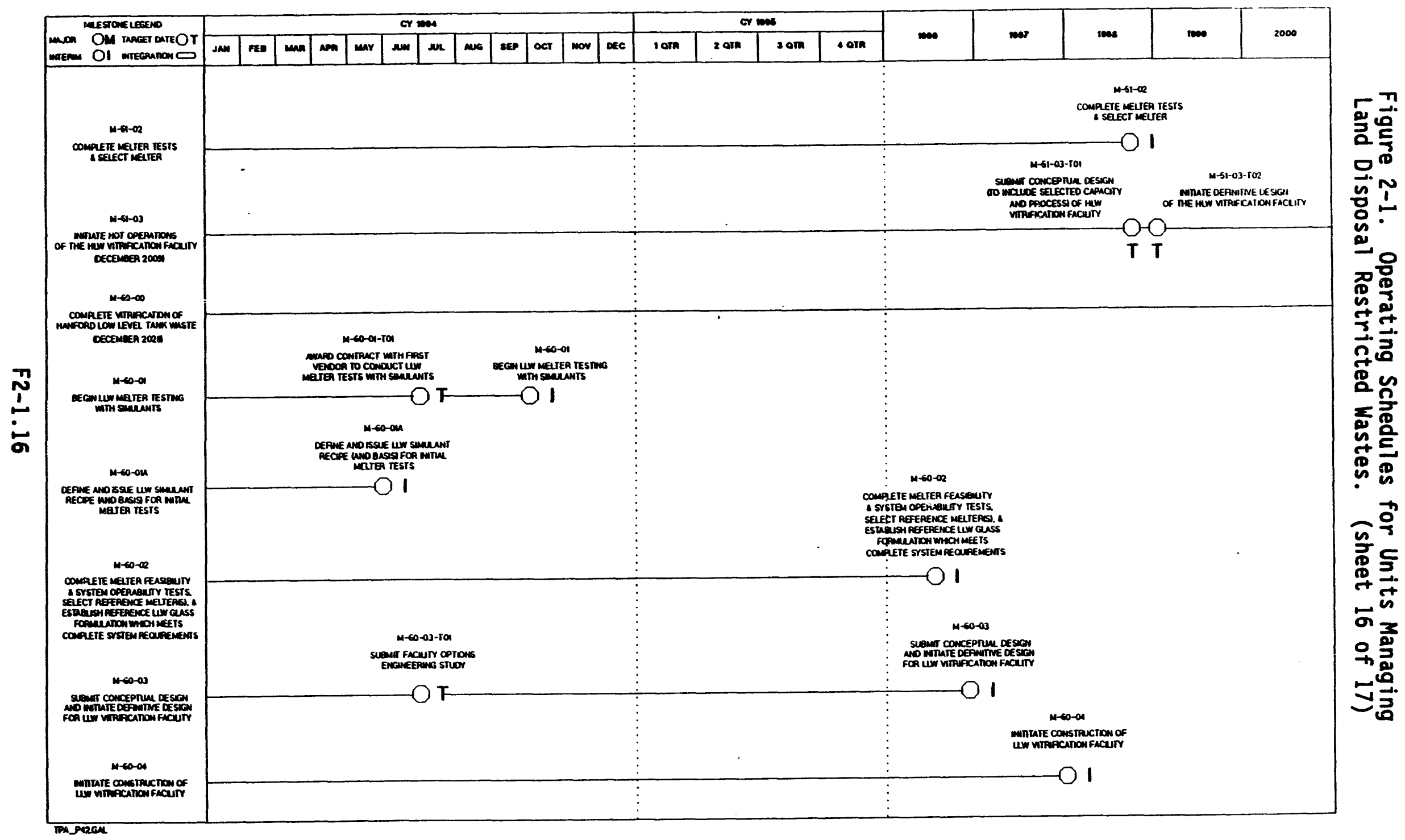




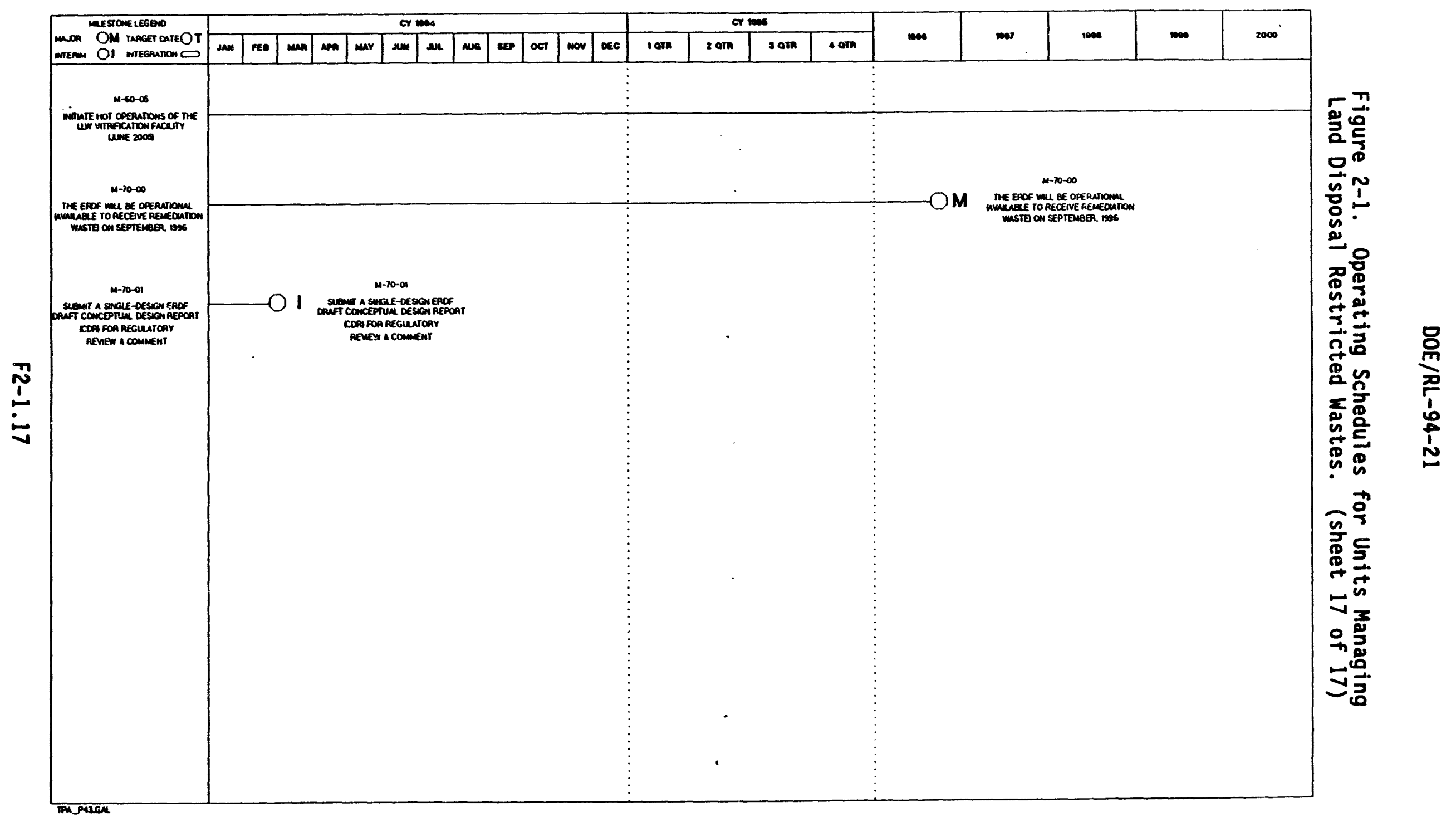


DOE/RL-94-21

Figure 2-2. Hanford Tank Waste Remediation System.

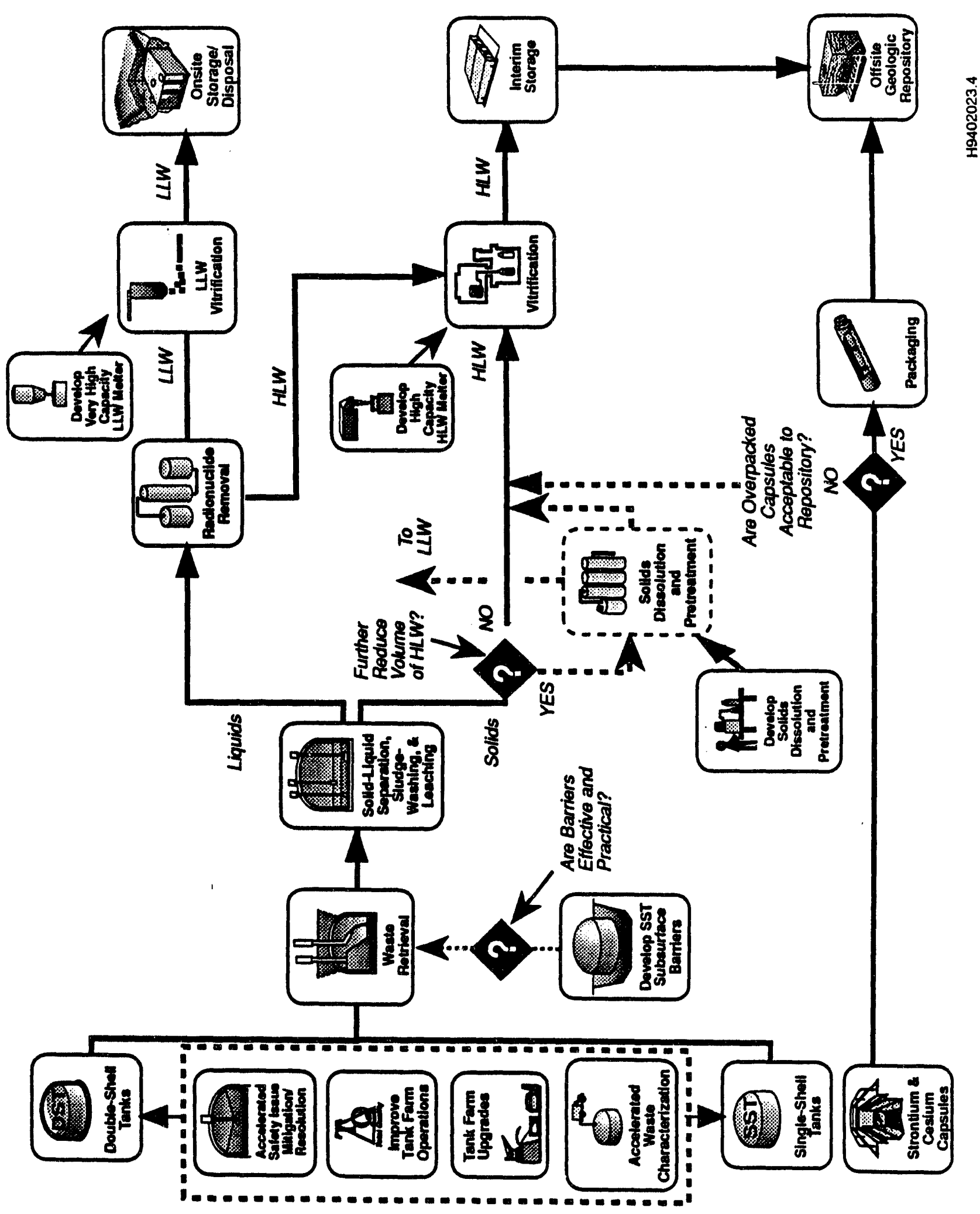


Figure 2-3. Plutonium-Uranium Extraction Aging Waste Treatment Flow Diagram.

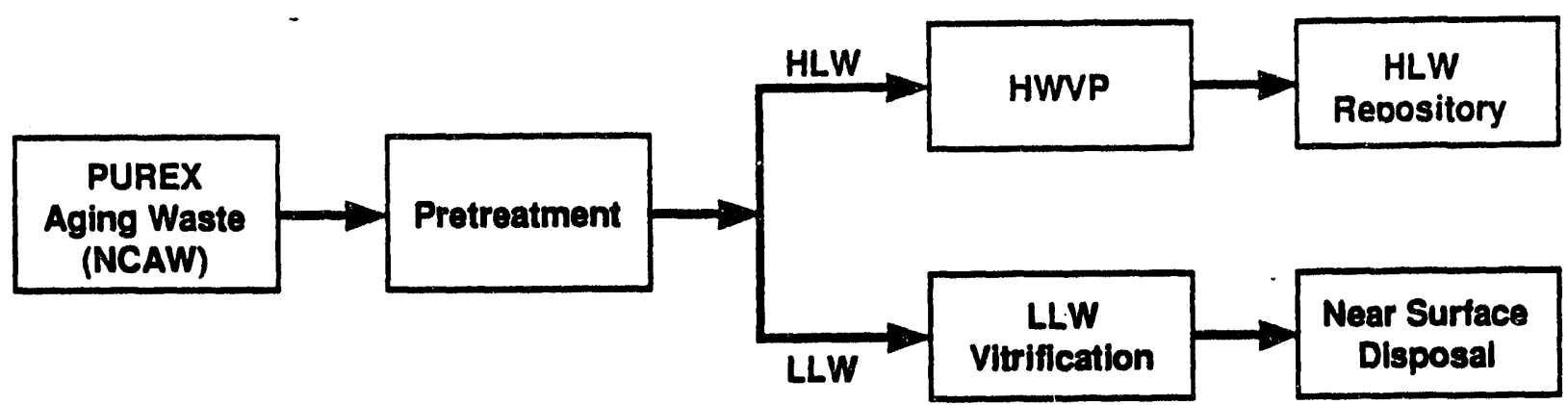

Legend

HLW High-level waste

HWVP Hantord Waste Vitrification Plant

LLW Low-level waste

NCAW Neutralized current acid waste

PUREX Plutonium-Uranium Extraction (Plant)

79108088.22 
Figure 2-4. Central Waste Complex Stored Waste, Retrievably Stored Waste, 183-H Solar Basin Waste, and 303-K Waste Treatment Flow Diagram.

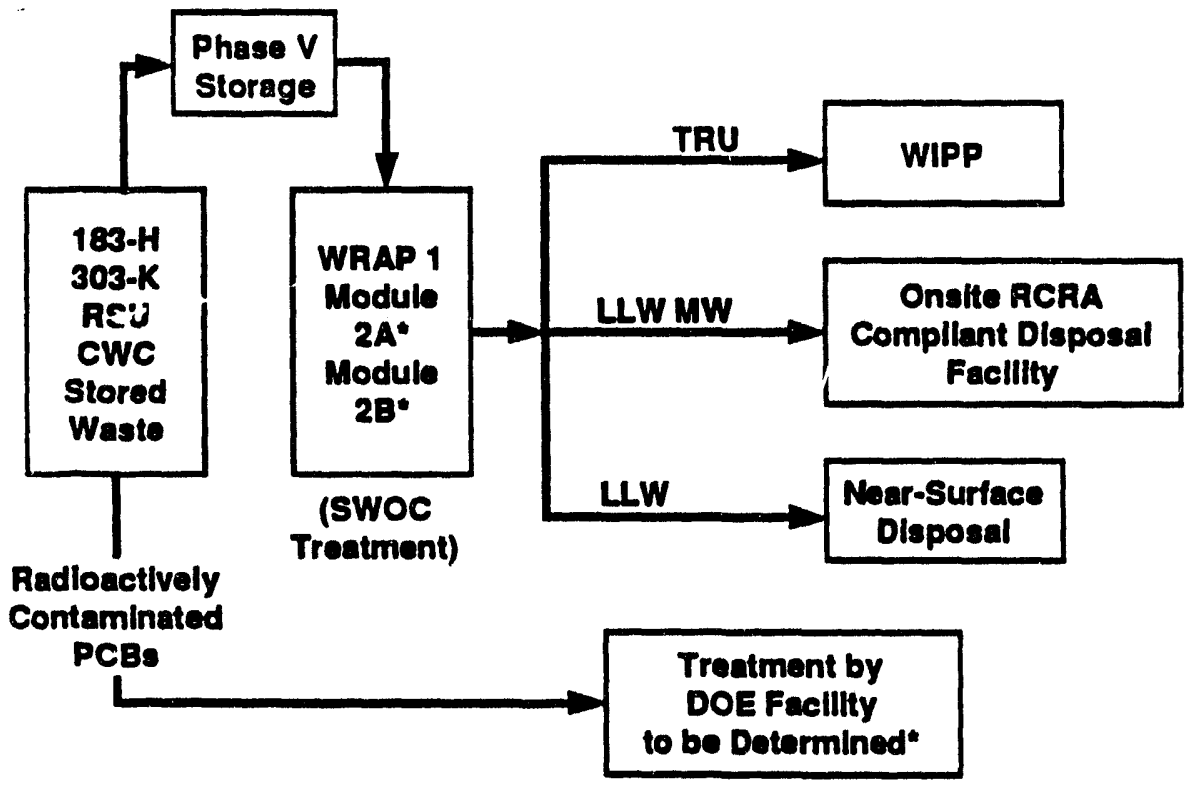

Legend

CWC Central Wasto Complex

DOE U.S. Department of Energy

LLW low-level waste

MW mixed waste

PCB polychlorlnated blphenyl

RCRA Resource Conservation and Recovery Act

* Onalte or private vendor supplled treatmont.
RSU retrlevable storage units

sWoc Solld Waste Operations Complex TAU transuranic

TRUSAF Tranauranic Waste Storage and Assay Facllty

WPP Waste Isolation Pllot Plant

WRAP Waste Recelving and Processing

79108088.25 


\subsection{INDIVIDUAL WASTE STREAM INFORMATION}

\subsection{DOUBLE-SHELL TANK WASTE}

Most DST waste was generated during the past production of nuclear materials. The DST waste is stored as alkaline liquids and solids in double-shel1 underground storage tanks in the 200 Areas of the Hanford Site. Twenty-eight DSTs store 96,967 cubic meters of waste as of December 31, 1993 (WHC 1993e). Two of these DSTs contain PUREX aging waste and are addressed separately in Section 3.2.

The DST waste is (or has been) generated from the PUREX process, B PIant operations, the PFP, research and development programs, laboratories, and decontamination of plants and equipment. Liquid supernatant and interstitial liquids from SSTs also are pumped to DSTs for storage.

Treatment plans are to recover the contents of the tanks and immobilize them for disposal. The TRU and high-level fractions will be vitrified for disposal in a geologic repository; the low activity fraction will be vitrified for disposal near-surface onsite.

\subsubsection{Generation}

The DST waste has been generated by operations in the 100,200,300, and 400 Areas of the Hanford Site. The first DSTs were constructed in 1970 and the newest DSTs were completed in 1986. Projected generation rates for DST waste fluctuate depending on the operating schedules of the waste generating units. The start-up of planned treatment and disposal units will eventually decrease the current and future DST waste volumes.

3.1.1.1 Process. The tanks contain waste from current operations and waste from past chemical separations processes. The major contributors to the waste stored in DSTs are discussed in the following sections. All waste streams transferred to the DSTs for storage are treated with sodium hydroxide and sodium nitrite to minimize tank corrosion. In addition to newly generated waste, liquid waste stored in SSTs also is transferred to the DSTS. This waste originated from the same sources as that stored in the DSTs. These sources include the PUREX PIant, the PFP, and B Plant chemical processes as well as bismuth phosphate separations, uranium recovery, and reductionoxidation extraction processes. Descriptions of these processes are included below (DOE 1987).

Liquid waste streams destined for DSTs from current operations can be classified into four waste categories. These categories and examples follow:

1. Safety--Streams that are required to prevent hazards to personnel or equipment. Examples: PUREX criticality drains must be tested to prevent violation of criticality specifications; B Plant railroad tunnel must be washed down to reduce exposure to personnel. 
2. Regulatory--Required by a regulatory body. Example: the aging waste ventilation system condensate could exceed regulatory 1 imits for crib discharge and be sent to DSTs.

3. Tri-Party Agreeinent--Waste streams that are required to support the Tri-Party Agreement. Examples: Remaining SST wastes are to be pumped to DSTs to meet Tri-Party Agreement milestones for SST stabilization; S Plant laboratory wastes are generated from sampling to support Tri-Party Agreement activities.

4. Miscellaneous/Production--Miscellansous streams in support of Haniord Site program activities. Example: waste generated in cleaning the 400 Area Interim Examination and Maintenance Cell (IEMC) are required to support the fusion program or Argonne National Laboratory.

As a result of the delay in the restart of the 242-A Evaporator and the shortage of DST space, waste minimization 1 imits have been set based on categories 1-3. Category 4 wastes must be reviewed and approved by the Tank Space Management Board for acceptance.

Characterization and waste volume information for both DSTs and SSTs is contained in the document A History of the 200 Area Tank Farms (WHC 1990e).

3.1.1.1.1 The PUREX Process. The PUREX process was a solvent extraction process that used a tributyl phosphate in a kerosene-like solvent for recovering uranium and plutonium from nitric acid solutions of irradiated uranium. Laboratory waste and flush water also were sent to the DSTs from the PUREX PIant. The PUREX Plant began operation in 1956 and operated intermittently. It is currently beginning shutdown activities.

\subsection{Plutonium Fintshing Plant. In 1949 the PFP began converting} plutonium in solution to plutonium metal. This historic waste stream was high in metallic nitrates. The process comprises precipitation, solvent exchange, and ion exchange wastes. The current waste stream generated from the PFP is a low-salt stream from operating the building systems and from laboratory operations. High-salt streams are generated along with the low-salt stream during plutonium reclamation. Liquid wastes averaging $4.5 \%$ solids are sent to DSTs and average about $15 \mathrm{~L} / \mathrm{h}$. Similar liquid wastes, when the facility is operating, from plutonium reclamation average about $270 \mathrm{~L} / \mathrm{h}$.

Table 3-1 provides estimated PFP radioactive liquid waste compositions. The stream volumes and concentrations are estimated values based on process knowledge. Two batches of waste have been characterized to date, but complete results are not yet available. Characterization by analysis of PFP liquid waste that is to be transferred to DSTs is a requirement based on comments provided by EPA.

3.1.1.1.3 Bismuth Phosphate Separations. Beginning in the early 1940s, $B$ Plant and T Plant separated plutonium from uranium in irradiated fuel by coprecipitation with bismuth phosphate from a uranyl nitrate solution. The plutonium was further separated from fission products by successive precipitation cycles using bismuth phosphate and 1 anthanum fluoride. Waste 
containing uranium, acid, and many of the fission products was neutralized and stored in underground SSTs. This separation process was used from 1943 to 1957 .

The bismuth phosphate metal wastes were initially stored in separate SSTs; however, the metal waste was reprocessed to recover the uranium and the supernatant was scavenged and disposed to the cribs, leaving very little original metal waste remaining in the SSTs. In addition, through the years waste management operations have created a complex intermingling of the tank wastes.

3.1.1.1.4 Uranium Recovery Process. Uranium in process waste was mined from the SSTs by sluicing, dissolved in nitric acid, and processed through a solvent extraction process using tributyl phosphate in a kerosene-like solvent. The acid waste from the uranium recovery process was made alkaline and returned to SSTs. The recovery process, which operated from 1952 to 1958 in U Plant and from 1956 to 1958 in PUREX Plant, resulted in an increase in the volume of nonradioactive salts and a small increase in waste volume.

The uranium recovery process operated in $U$ PIant and at PUREX Plant were similar in that they used tributyl phosphate as the solvent; however, there were significant differences between the two processes. The wastes produced by the process in U Plant recovered uranium from bismuth phosphate metal wastes and discarded the fission products and residual plutonium. The process in U Plant also produced relatively dilute HLW, approximately 19 liters of waste per kilogram of uranium processed. The PUREX Plant process recovered uranium and plutonium in addition to separating the fission products. The PUREX process produced a much more concentrated high-level waste product than the process in $U$ Plant, approximately 0.2 liters per kilogram of uranium processed, and the waste was more radioactive, because the fuel was irradiated for a longer period of time.

No SSTs received acidic wastes or purely nonradioactive salts from these processes. The wastes were all neutral or alkaline in nature and the nonradioactive materials were ultimately mingled with radioactive materials.

A significant increase in the volume of waste resulted from the uranium recovery process in $U$ Plant. The process efficiently recovered uranium from the bismuth phosphate metal waste; however, it generated about 2 gallons of waste for every gallon of bismuth phosphate metal waste processed. This increase in waste volume was the rationale for the ferrocyanide scavenging campaign. It was necessary to reduce the occupied waste tank volume, and the ferrocyanide scavenging decontaminated the waste sufficiently to enable disposal to the cribs.

3.1.1.1.5 Reduction-0xidation Process. The reduction-oxidation process used a continuous solvent extraction process to extract plutonium and uranium from dissolved fuel in a hexone solvent. The slightly acidic waste stream contained the fission products and large quantities of aluminum nitrate. This waste was neutralized and stored in SSTs. The 202-S Plant operated between 1951 and 1967. 
3.1.1.1.6 Cesium and Strontium Recovery. Past operations in B Plant for recovery of cesium and stront fum from waste were a main source of DST waste. This waste is known as complexant concentrate.

3.1.1.1.7 Other Contributors to Double-Shell Tank Naste. Cleaning solutions and other miscellaneous waste are chemically adjusted to minimize tank corrosivity and transferred to DSTs for storage. The waste includes the following:

- Spent cleaning solutions from decontamination and ion exchange regeneration at the 100 Area

- Waste from decontaminating and decommissioning tools and equipment

- Laboratory waste from the 200 Area

- Fuels fabrication waste from the 300 Area

- Miscellaneous waste from the FFTF operations in the 400 Area.

Additional detail can be found in the DST Part B Permit Application (RL 1991a).

3.1.1.2 Generation. The DSTs do not simply accumulate and store waste; the tanks are a waste-handling system. The inflows to the system include supernate and interstitial liquids pumped from SSTs, laboratory wastes, and waste from inactive facilities. Outflows include evaporation and future pretreatment and vitrification processes. Evaporation decreases the DST waste volume; pretreatment and vitrification remove DST waste and prepare it for disposal.

Projected DST waste generation through 1999 is shown in Figure 3-1 in terms of tank space used versus available space. The average generation rate for DST waste is about 10,800 cubic meters per year before evaporation. This generation rate is based on waste generation projections through 1998 (Table 2-1).

\subsubsection{Characterization}

The wastes in DSTs consist of solids and liquids. Typically the solids fraction has settled out as a sludge layer. The wastes are LLW, TRU waste, and $H L W$, and designated as ignitable, corrosive, toxic, persistent, and carcinogenic extremely hazardous waste. Because of heavy metals contamination, DST waste also is designated as toxic by the TCLP.

This section summarizes process knowledge and sample analysis for the contents of the DSTs. The assumed waste designations and their bases are described, and schedules for further analysis are given.

3.1.2.1 Process Knowledge. Several processes contribute to DST waste, as described in Section 3.1.1.1. Waste management practices, including evaporation of tank contents, have intermingled the various types of waste. 
This intermingling precludes a detalled, quantified characterization of the tank contents based strictly on process knowledge. Instead, the DST waste is described qualitatively based on generation data and sample analysis.

Stratification and segregation have occurred in the tanks as solids have settled out. The consistency of the waste ranges from liquid supernatant to a thick sludge to crusts formed as a top layer.

The major constituents of DST waste are water and sodium salts of aluminate, nitrate, nitrite, phosphate, hydroxide, carbonate, and sulfate. Some calcium and potassium salts also are present. Complexed waste in the DSTs contains sodium salts of the chelating agents ethylenediamine-tetraacetic acid and n-hydroxyethylethylenediamine-tetraacetic acid. There also may be detectable concentrations of halogenated and nonhalogenated organic compounds and heavy metals such as lead, chromium, and cadmium.

In addition, DST waste may be categorized into several types which each have a specific history and character. These wastes types, defined beicw, include:

- Double-Shell Slurry/Double-Shel1 Slurry Feed (DSS/DSSF)

- Neutralized Current Acid Waste

- Neutralized Cladding Removal Solids Waste

- Plutonium Finishing Plant Waste

- Complexant Concentrate Waste.

3.1.2.1.1 Definition of Double-Shell Slurry Feed and Double-Shell Slurry Waste. Double-shell slurry feed is generated by concentrating the dilute waste streams generated by the operating plants to conserve storage space. Double-shell slurry is generated by further concentration of DSSF.

Double-shell slurry feed and DSS are concentrated waste types generated by the evaporation of dilute noncomplexed waste streams to conserve tank space. The DSSF waste type has been evaporated up to, but not beyond, the sodium aluminate phase boundary; therefore, it contains no aluminate solids. Double-shell slurry is a more concentrated waste form that is produced by further evaporation of DSSF past the aluminate boundary. Double-shell slurry contains aluminate solids and has a much higher viscosity, which makes retrieval from tanks more difficult and costly. of DSSF.

There are currently 3,607 cubic meters of DSS and 15,702 cubic meters

3.1.2.1.2 Definition of Meutralized Current Acid Waste. The NCAW is al so known as PUREX aging waste. Further discussion of NCAW is contained in Section 3.2. 
3.1.2.1.3 Definttion of Noutralized Cladding Removal Solids Waste. Cladding removal waste results from dissolving the zircaloy $\mathrm{cladding}$ of irradiated nuclear fuel from $N$ Reactor. Neutralizing the waste precipitates most of the zirconium and creates a slurry. The resulting stream is called neutralized cladding removal waste (NCRW).

3.1.2.1.4 Definition and Treatment of Plutonium Finishing Plant Waste. The PFP waste originates from the conversion of plutonium nitrate to oxide or metal and includes TRU laboratory waste and high-salt solvent extraction waste. Projected generation of this waste through 1995 is 1,150 cubic meters. Current inventory in storage is estimated at 390 to 503 c/ubic meters. This is stored in Tank 241-SY-102 where it is blended with other 200 West Area wastes.

3.1.2.1.5 Dofinition and Treatment of Complexant Concentrate Maste. The complexant concentrate results from the concentration of waste containing large amounts of organic complexing agents. The organic complexing compounds were introduced to the waste during strontium recovery at B Plant. No future generation of this waste is $p l a n n e d$.

\subsubsection{Sample Analyses. Samples of the DSTs have been analyzed per EPA} SW-846 methodology (EPA 1986). Because no one DST constitutes a "representative" tank, the analytical data from these samples are presented in Table 3-2 as ranges of values for tank composition.

3.1.2.3 Waste Designation and Basis. All waste stored in DSTs is designated corrosive dangerous waste (D002) because it has been treated with sodium hydroxide to raise the $\mathrm{pH}$ above $\mathbf{1 2 . 5}$ in preparation for tank storage.

The DST waste is assumed to be extremely hazardous waste (WTO1) for toxicity based on the concentration of chemicals in the waste. The waste may exhibit the characteristic of ignitability (DOO1) as identified in WAC 173-303-090 because of the presence of oxidizers such as nitrate and nitrite. In accordance with Tri-Party Agreement Milestone M-44-00, the data quality objectives process will be used to establish the necessary sampling and analyses for designation, as well as to establish if all applicable treatment standards for waste are being met. The process will al so determine for ignitable and corrosive wastes which underlying hazardous constituents must be quantitized to determine compliance (per 58 FR 29860). The DST waste also is suspected to contain spent solvents including 1,1,1-trichloroethane, hexone, acetone, and oresylic acid (waste codes F001 through F005 are assigned). The DSTs contain waste that meets TCLP criteria for heavy metals contamination: arsenic (D004), barium (D005), cadmium (D006), chromium (D007), lead (D008), mercury (D009), selentum (D010), and silver (D011). The waste also is carcinogenic (WCO1, WCO2) and persistent (WPO1, WPO2).

The DSTs do not contain waste listed on the dangerous waste source list of WAC 173-303-082.

Radioactive constituents include americium-241, carbon-14, cesium-137, cobalt-60, curium-244, iodine-129, neptunium-237, plutonium-239 and -240 , ruthenium- and rhodium-106, selenium-79, strontium-90, technetium-99, and tritium. 
3.1.2.4 Uncertainty of Waste Designation. The waste codes previously assigned are considered accurate, but have been assigned based on 1 imited analytical data. Additional waste codes may be added or deleted based on the ongoing characterization program.

3.1.2.5 Schedule for Further Characterization. Sampling and analysis of the DST contents is under way and will continue based on prioritization through the Systems Engineering approach.

There are two types of samples: cores and bottles-on-a-string. The analytical procedures used by the two onsite laboratories to characterize the DST waste samples are based on methods and techniques found in the EPA Test Methods and Evaluation of Solid Waste (SW-846). However, some of these procedures have been modified in terms of sample sizes and preparation techniques to reflect the radioactive nature of the waste samples and the complex constituent matrix. A comprehensive list of the chemical analyses, radionuclides, and physical measurements to be included in the DST

characterization effort will be found in the Tank Waste Analysis Plan.

\subsubsection{Storage}

This section describes DST storage and assesses its compliance with existing regulations.

3.1.3.1 Storage Unit and Capacity. There are 28 DSTs, each with a 4,300-cubic-meter capacity. Four of these DSTs are equipped to manage PUREX aging waste and are addressed separatefy in Section 3.2. The 28 tanks are located in 6 tank farms in the 200 Areas of the Hanford Site.

3.1.3.2 Amount in Storage. As of December 31, 1993, the tanks held 89,756 cubic meters of waste (WHC 1993e). This does not include PUREX aging waste (Chapter 3.0, Section 3.2). Projections indicate that the DSTs could be filled to capacity in 1994 based on current expected generation rates. The construction of up to six new DSTs is proposed to relieve the 1 imitations.

3.1.3.3 Storage Compliance Assessment. The DSTs were reviewed for compliance with interim status dangerous waste regulations in accordance with Tri-Party Agreement (Ecology et al. 1992) Milestone M-21-00. The assessment for compliance with interim status regulations noted the following areas of noncompliance:

- Inspection plan

- Waste analysis plan

- Waste characterization

- Training plan. 

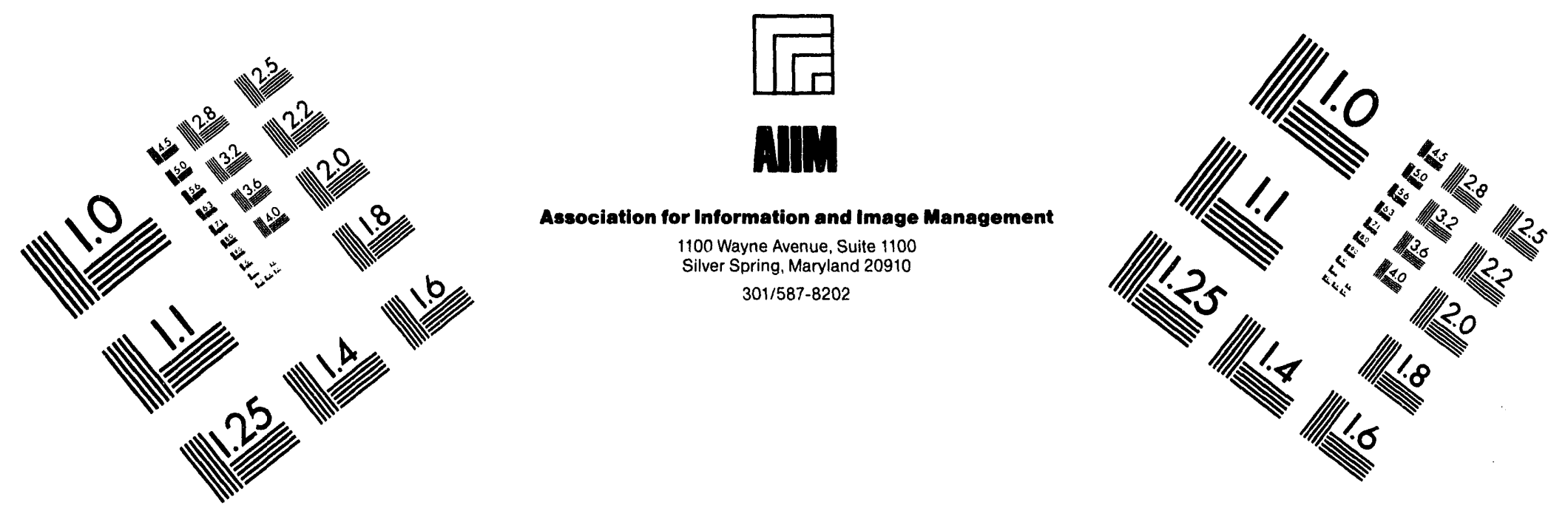

\section{Centimeter}

$\begin{array}{llllllllllllllll}1 & 2 & 3 & 4 & 5 & 6 & 7 & 8 & 9 & 10 & 11 & 12 & 13 & 14 & 15 & \mathrm{~mm}\end{array}$

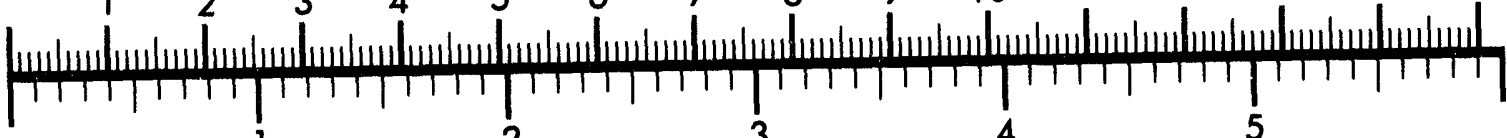

Inches
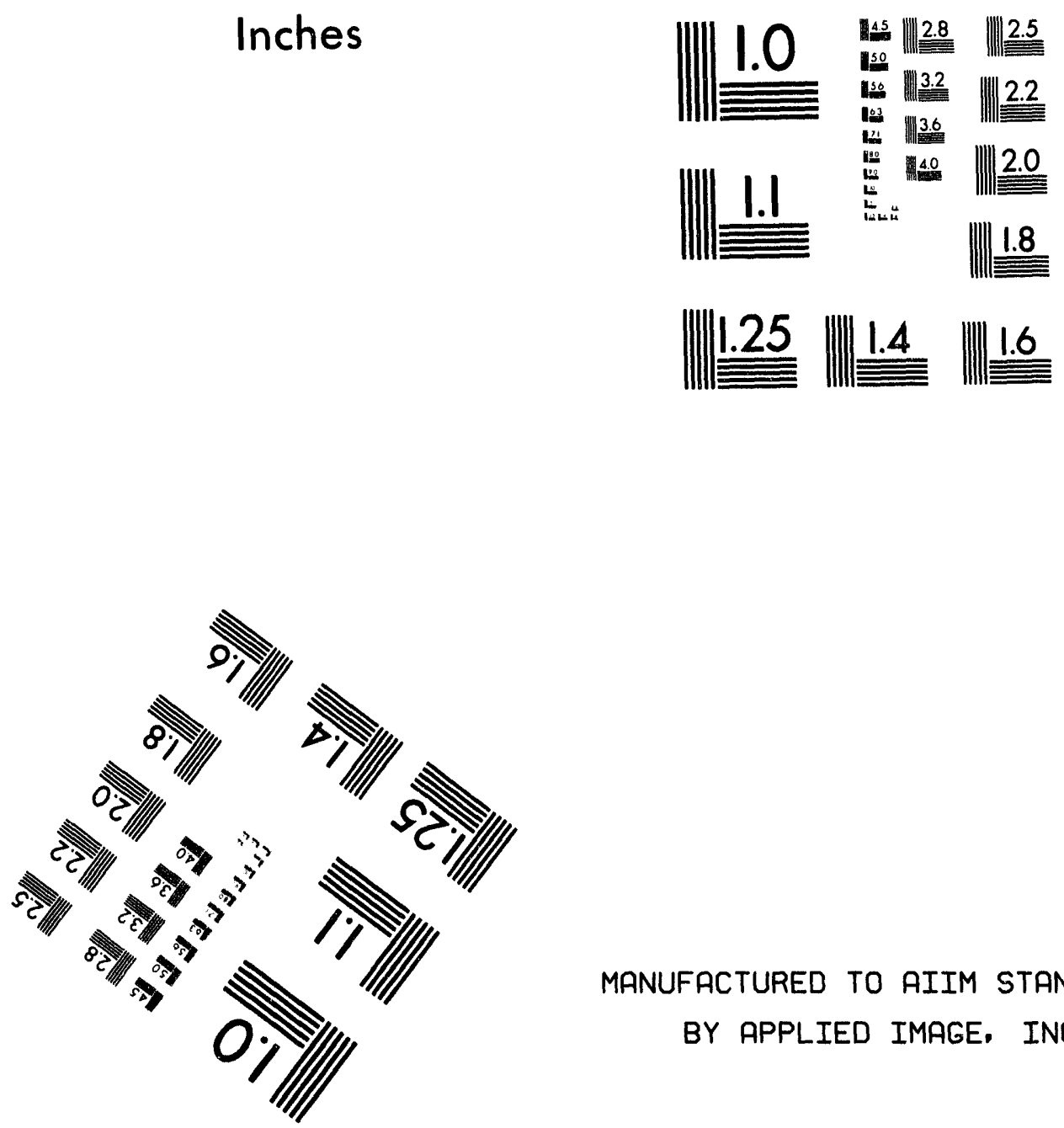

MANUFACTURED TO AIIM STANDARDS

BY APPLIED IMAGE, INC.

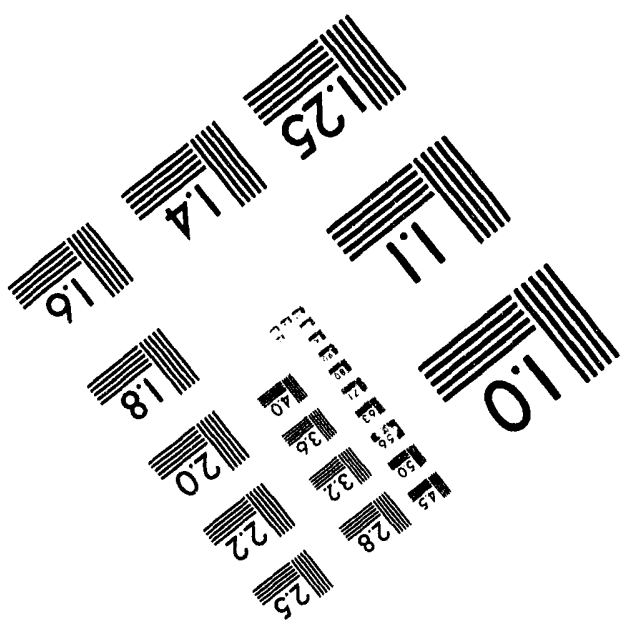



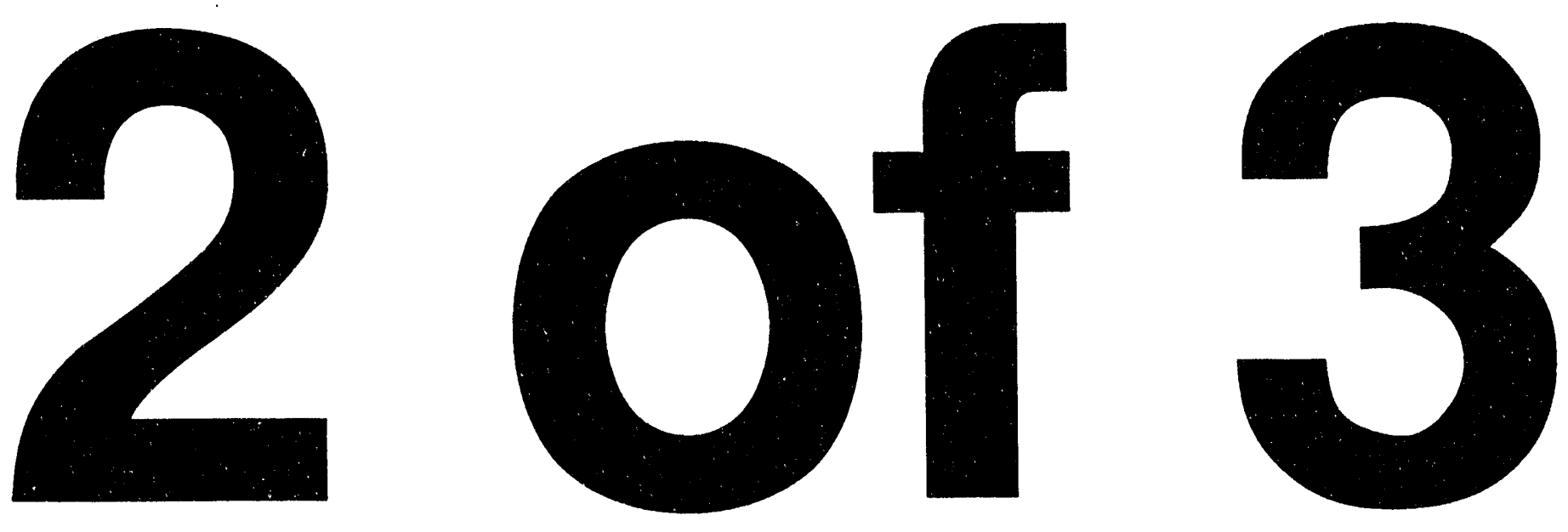
Compliance action schedules for DSTs are being negotiated in the Tri-Party Agreement. Interim status compliance for the items 1 isted is completed. Additional DST actions may be required after December 1990 . These actions may include the following:

- Recordkeeping system modifications

- Provision of secondary containment for ancillary equipment

- Development of additional leak detection systems

- Development of a closure plan.

\subsubsection{Treatment}

This section discusses current and proposed treatment of DST waste.

3.1.4.1 Current Treatment. The 242-A Evaporator reduces the DST waste volume by evaporative concentration (Chapter 3.0, Section 3.4.1.1). It began operating in 1977 and has evaporated more than 246,000 cubic meters of water from the DST stored waste.

3.1.4.2 Proposed Treatment. In addition to those wastes currently being generated for DST storage (supernate and interstitial liquids pumped from SSTs, laboratory wastes, and waste from inactive facilities), wastes currently stored in the DSTs will be treated and disposed by means of the same processes and facilities recently adopted by the Tri-Party Agreement. The DST waste will be retrieved, pretreated, and solidified for disposal. Pictorial flow diagrams are shown in Figures 2-2 and 3-2.

Pretreatment separates the DST waste into a LLW and HLW/TRU fraction so that the bulk of the radionuclides are in the HLW. The HLW stream will then be additionally treated to reduce its volume and increase radionuclide loading if necessary. The LLW will have enough radionuclides removed so that it will meet the Nuclear Regulatory Commission's "incidental waste" classification and the DOE's as low as reasonably achievable (ALARA) policy.

Processes requiring limited development will be used to the extent practical to accomplish the pretreatment function and reduce the HLW volume to be vitrified. For the LLW pretreatment, these technologies will focus primarily on removing cesium and strontium from the waste streams to be treated. For the HLW pretreatment, technologies will focus on producing a stream that will create a low-volume, high loading glass. Development of enhanced technologies, which are expected to include sludge washing, selective leaching, and blending, will continue to be pursed. Organic destruction and waste specific pretreatment technologies will continue to be developed until implemented or determined unnecessary.

In respective facilities, both the $L L W$ and $H L W$ fractions will be vitrified, a process that will destroy or extract organic constituents to below treatment standards, neutralize or deactivate dangerous characteristics, and immobilize toxic metals. The LLW fraction will be disposed near-surface 
onsite in a retrievable form. The vitrified HLW stream will be stored onsite until the Geologic Repository Program is available to receive the waste for disposal.

\subsubsection{Treatment Alternatives and Accelerated Treatment. Alternative} treatments are discussed where applicable in Section 3.1.4.2. In addition, a tank waste technical options report has been issued (WHC 1992C) that presents a number of alternatives for remediating DSTs. Alternative pretreatment technologies are discussed further in WHC (1993a).

Treatment of DST waste is on a schedule based primarily upon Tri-Party Agreement milestones $\mathrm{M-50-00}$ (pretreatment), M-60-00 (pretreatment) and M-51-00 (HLW vitrification). (Refer to Figure 2-1 for details.) Because of budget limitations, accelerating treatment beyond these milestone dates is not realistic.

\subsubsection{Waste Reduction}

Currently 11 major plants or programs generate DST waste. Annual waste generation for FY 1990 through 1993 is listed in Table 3-3. Total waste generation was reduced by $60 \%$ from 1990 to $1991,54 \%$ from 1991 to 1992 , and 26\% from 1992 to 1993. (The latter figure does not include water additions used in evaporator tests: see Table 3-3 for details.) The caption labeled "SST to DST Pumping" refers to pumping liquid waste from SSTs to meet Tri-Party Agreement milestone M-41-00 requiring all SSTs to be stabilized by the end of FY 2000. Waste reduction activities (current and planned) are outlined for each unit in the Annual Report of Tank Waste Treatability (WHC 1993d). The four activities include minimizing flush volumes and frequency, pretreating waste (e.g., destroying ammonia), modifying processes, and recyciling streams.

Dilute waste received at the DSTs will be concentrated by the 242-A Evaporator, further reducing the waste volume by 30 to $95 \%$. In an average year, projected volumes of dilute waste will be reduced by approximately $71 \%$.

\subsubsection{Variances, Exemptions, Time Extensions}

The DST waste consists of LLW, TRU Waste, and HLW containing dangerous waste constituents. The DST waste is restricted from land disposal because it contains solvent waste (40 CFR 268.30), Cal ifornia List waste (40 CFR 268.32), and waste covered by the Third-Third Promulgation (55 FR 22520).

The national capacity variance for Third-Third Waste (55 FR 22520) provided for a 2-year national capacity variance, from the LDR for Third-Third mixed waste expiring on May 8, 1992. This variance allowed continued storage of these wastes. A Federal Register notice dated May 26, 1992, stated that EPA could not grant a case-by-case extension for Third-Third mixed waste because of questions regarding the DOE's demonstration that they have entered into binding contractual commitments to construct or otherwise provide treatment capability. A final decision has yet to be made. This extension would allow DOE to store LDR mixed wastes until May 8, 1993; this date could 
be extended for up to an additional year. The Tri-Party Agreement will allow continued storage of these wastes in accordance with the schedules in the Tri-Party Agreement. The Federal Facility Compliance Act of 1992 also allows DOE facilities to store their wastes for 3 years if compliance with the Act's provisions for providing waste inventory and treatment plans are met.

The Tri-Party Agreement provides for continued storage of California List (40 CFR 268.32) and solvent waste (40 CFR 268.30) until treatment capacity is developed for these wastes. The agreement requires treatment and disposal capacity for these wastes to be developed on the following schedule:

- Low-Level Waste--Disposal of treated waste by viirification as soon as sufficient quantities are available to facilitate proper treatment and disposal, in accordance with the schedule defined in the Tri-Party Agreement that requires all LLW contained in DSTs and SSTs to be vitrified by 2028.

- Transuranic Waste--Treatment schedules for TRU waste stored in the DSTs (and SSTs) coincide with those for the treatment of HLW, discussed below.

- High-Level Waste--Treatment of waste will begin as soon as the HLW vitrification facility has been constructed and sufficient quantities of pretreated waste are available (scheduled for 2009 per the Tri-Party Agreement). Disposal is intended for a national HLW geologic repository, with an uncertain start-up date.

If additional variances, exemptions, or time extensions are required as a result of delays in the development of treatment, storage, or disposal capacity, they will be applied for in accordance with the procedures detailed in the Tri-Party Agreement or regulations.

\subsection{PUREX AGING WASTE}

The aging waste storage unit comprises four DSTs in the 241-AY (Tanks 241-AY-101 and -102 ) and 241-AZ (Tanks 241-AZ-101 and -102) tank farms in the 200 East Area of the Hanford Site. Two latter DSTs, 241-AZ-101 and -102 , presently hold a mixture of solids and supernate aging HLW (from the PUREX Plant).

Aging waste from the PUREX Plant comes from the first decontamination solvent extraction column in the PUREX solvent extraction process. The feed to the extraction column is irradiated fuel elements dissolved in nitric acid. The extraction column separates the uranium and TRU products from the majority of the fission products. The fission products are contained in the aqueous nitric acid phase from the extraction column. The aqueous phase is concentrated to recover nitric acid and reduce volumes, and the concentrated stream is sampled. If it is determined to be a waste, based on sample analysis, it is treated with sugar to destroy the majority of the nitric acid. Sodium hydroxide is added to meet storage tank specifications and the waste is transferred to the aging DSTs for storage. As of December 31, 1993, a total of 7,211 cubic meters of PUREX aging waste was in storage. 
The waste stream is considered corrosive and toxic and has designated EPA waste codes of D002, D006, D007, and D008. The waste stream will be treated to separate the HLW from the LLW in the DST pretreatment facility. The low-level fraction will be vitrified and disposed onsite and the high-level fraction will be vitrified and stored until a repository is available.

\subsubsection{Generation}

This section describes the waste generation process. The PUREX P1ant received official notification to begin shutdown on September 24, 1992. Aging waste will not be generated in the future. Deactivation planning for the PUREX plant is underway. When estimated future volumes of other waste types are generated, the information will be added to this report.

The PUREX Plant received irradiated zirconium clad fuel from $N$ Reactor, removed the cladding from the fuel, and dissolved the fuel in nitric acid. The dissolved fuel was processed through several solvent extraction steps to separate the plutonium, uranium, and neptunium from the fission products contained in the fuel. The aging waste contains the majority of the fission products from the fuel and is generated from the aqueous siream from the first extraction column.

\subsubsection{Characterization}

This section discusses the available waste characterization information. Information based on process knowledge and sample analysis is provided along with the waste designations and their bases, the uncertainty related to the designation, and the schedule for further analysis.

3.2.2.1 Process Knowledge. The aging waste comprises water, aluminum hydroxide, sodium nitrate, sodium hydroxide, sodium fluoride, cadmium nitrate, sodium nitrite, corrosion products, and the majority of radionuclides from $N$ Reactor fuel. Past practice (before 1989) was to recycle process samples analyzed in the laboratory back to the process system, which may have resulted in some of the chemicals added to the samples entering the aging waste. The presence of these chemicals in the aging waste never has been confirmed by sample analysis.

3.2.2.2 Sample Analyses. The composition of PUREX Plant NCAW is given in Table 3-4. The results of sample analyses per EPA SW-846 methodology (EPA 1986) of the PUREX aging waste stored in the DSTs are given in Table 3-5.

3.2.2.3 Waste Designation and Basis. The NCAW stream contains excess amounts of sodium hydroxide $(0.8 \mathrm{M}$ ) making the waste corrosive dangerous waste (D002) and LDR (52 FR 22520). Based on equivalent concentration calculations, there are sufficient concentrations of sodium nitrate and sodium hydroxide to make the aging waste toxic dangerous waste (WTO1). In addition, there are sufficient quantities of heavy metals to designate the NCAW as a toxic, as determined by the TCLP, for cadmium (D006), chromium (D007), and possibly lead (D008). 
3.2.2.4 Uncertainty of Waste Designation. Based on sample data from Tanks 241-AZ-101 and -102 (Table 3-5), the waste designation is correct.

3.2.2.5 Schedule for Further Characterization. Three core samples have been taken and characterized to date; one supernate sample is planned for FY 1994.

\subsubsection{Storage}

This section provides the volume currently in storage and assesses the compliance status of the storage unit.

3.2.3.1 Storage Unit and Capacity. The aging waste storage unit comprises four DSTs in the 241-AY and 241-AZ tank farms. Only the 241-AZ tank farm currently contains aging waste. The $A Y$ and $A Z$ aging waste tanks each have a maximum fill volume of 3,800 cubic meters. The use of air-lift circulators limits the working volumes to 3,700 cubic meters for these tanks. These circulators keep the supernate agitated and aid in heat removal from the tanks. The tanks also are equipped with steam coils to boil away water in the waste and a ventilation system that can handle large amounts of steam.

3.2.3.2 Amount in Storage. Tanks 241-AZ-101 and -102 contain approximately equal volumes totaling 7,211 cubic meters. The waste in these tanks is NCAW.

3.2.3.3 Storage Compliance Assessment. The PUREX aging waste is stored in the DSTs. The DSTs were reviewed for compliance with interim status dangerous waste regulations in. accordance with Tri-Party Agreement (Ecology et al. 1992) Milestone M-21-00. The results of the compliance assessment are provided in Chapter 3.0, Section 3.1.3.3.

\subsubsection{Treatment}

This section discusses the current and proposed waste treatment processes.

3.2.4.1 Current Treatment. Currently the aging waste is being stored pending pretreatment and vitrification.

3.2.4.2 Proposed Treatment. The NCAW will be pretreated in preparation for disposal to remove and concentrate as many radionuclides as possible into a HLW stream and produce a LLW byproduct stream. The LLW fraction will be vitrified and disposed onsite. The HLW fraction, which may require additional pretreatment in the HLW vitrification facility to reduce its volume, will incorporate TRU waste and HLW into a glass matrix for long-term storage and ultimate disposal.

3.2.4.3 Treatment Alternatives. Any applicable treatment alternatives are discussed in Section 3.1.4. 
DOE/RL-94-21

3.2.4.4 Accelerated Treatment. Treatment of aging waste is on a schedule based primarily on Tri-Party Agreement Milestones M-50-00 (pretreatment), M-60-00 (LLW vitrification), and M-51-00 (HLW vitrification). (Refer to Figure 2-1 for details.) Because of budget limitations, accelerating treatment beyond these milestone dates is not realistic.

\subsubsection{Waste Reduction}

The production of HLW by the PUREX Plant was reduced from 9,800 kilograms per day of operation in 1985 to 4,900 in 1988. Minimization of aging waste was accomplished through increased process control of the aqueous stream concentration, better control of aluminum nitrate addition, and better control of sodium hydroxide addition to adjust waste stream $\mathrm{pH}$ to tank specifications. The minimization is graphically illustrated in Figure 3-3.

The following process improvements were implemented:

- Optimum control of the evaporator waste concentration overflow rate

- Reduction of the aluminum-to-fluoride ratio in the aluminum nitrate nonahydrate addition to the dissolvers during fuel processing.

On December 21, 1992, PUREX received official notification for shutdown and to proceed with terminal cleanout activities. Aging waste is no longer generated by the PUREX Plant.

\subsubsection{Variances, Exemptions, Time Extensions}

The PUREX aging waste consists of HLW mixed with dangerous waste constituents. The PUREX aging waste is a LDR waste because of both the Third-Third Promulgation (55 FR 22520) and the presence of California list constituents. The Tri-Party Agreement (Ecology et al. 1992) provides for continued storage of LDR waste until treatment capacity is developed for this waste. The agreement requires treatment and disposal capacity for this waste to be developed on the following schedule:

- Initiate pretreatment options by December 2004

- Initiate enhanced HLW pretreatment by June 2008

- Initiate LLW vitrification operations by June 2005

- Initiate HLW vitrification operations by December 2009

- Dispose of vitrified waste when repository opens.

The national capacity variance for Third-Third Waste (55 FR 22520) provided for a 2-year national capacity variance from the LDR for Third-Third mixed waste expiring on May 8, 1992. This variance allowed continued storage of these wastes. A Federal Register notice dated May 26, 1992, stated that EPA could not grant a case-by-case extension for Third-Third mixed waste because of questions regarding the DOE's demonstration that they have entered 
into binding contractual commitments to construct or otherwise provide treatment capability. A final decision has yet to be made. This extension would allow DOE to store LDR mixed wastes until May 8, 1993, and this date could be extended for up to an additional year. The Tri-Party Agreement will allow continued storage of these wastes in accordance with the schedules in the Tri-Party Agreement. The Federal Facility Compliance Act of 1992 also allows DOE facilities to store their wastes for 3 years if compliance with the Act's provisions for providing waste inventory and treatment plans are met.

If additional variances, exemptions, or time extensions are required as a result of dalays in the development of treatment, storage, or disposal capacity, they will be applied for in accordance with the procedures detailed in the Tri-Party Agreement or regulations.

\subsection{SINGLE-SHELL TANK WASTE}

The SST waste currently in storage is not a LDR waste because it was placed in storage before the effective date of the LDRs for mixed waste (November 27, 1987). Information on the SSTs is included only because when the waste is retrieved or transferred, it then becomes a LDR waste and this information will be required to identify treatment and disposal capacity requirements.

The SSTs are underground, reinforced-concrete, stee1-1ined tanks used for waste storage. These tanks have held chemically hazardous and radioactive waste generated as a byproduct of processing spent nuclear fuel for the recovery of plutonium, uranium, and neptunium beginning in 1944; additional tanks were constructed as required.

Liquid waste collection and storage in the SSTs continued until November 1980. The only material added to the SSTs since 1980 has been water, which was added to two tanks for evaporative cooling purposes. An interim stabilization program was initiated in 1968 to remove pumpable interstitial liquid and supernatant from the SSTs and transfer it to the DSTs. This program primarily is intended to reduce the leak potential of the SSTs and will be completed in 1996 (WHC 1990h).

The SSTs consist of 149 tanks containing approximately 139,500 cubic meters of waste. These tanks are located in 12 tank farms with 4 to 18 tanks each in the 200 Areas. The amount of waste contained in the tanks varies from 5 to $95 \%$ of each tank's capacity and varies in consistency from pumpable liquid to sludge to hard salt cake.

The SSTs have released an estimated $2,600 \mathrm{~m}^{3}$ of liquid to the soil column. However, after some tanks were declared to be leaking, cooling water may have been added to aid evaporative cooling. It is believed that some of this water did not evaporate and, therefore, went into the ground. As of October 1990, estimates for this additional water release ranged from 190 to 3,000 cubic meters. The past practice was to exclude the cooling water from the leak volume estimate. 
In addition, documents show that from 1946 to $1966,456,725$ cubic meters (120,661,000 gallons) of liquid wastes were intentionally discharged from SSTs at the Hanford Site directly to the ground on the 200 Areas plateau

(WHC 1991c). The majority of this waste was discharged from 1946 to 1958 as a result of the early plutonium and uranium recovery processes conducted in the 221-B Facility (B Plant), 221-T Facility ( $T$ Plant), and the 221-U Facility (U Plant). In addition, from 1960 to 1966 laboratory wastes from the 300 Area and equipment decontamination wastes from the 200 West Area were routed through SSTs before discharge to the ground. No wastes have been discharged intentionally to the ground from SSTs since 1966. Table 3-6 details the current estimates of releases.

\subsubsection{Generation}

This section describes the waste generation process. Also refer to Section 3.1.1.1 for additional information.

The waste has been generated through a variety of analytical, decladding, and separation processes and various associated sitewide operations. The SSTs received this waste from various Hanford Site activities before 1980 .

Waste currently stored in the SSTs was produced by four major chemical processing operations that were conducted from 1944 to 1980:

- The bismuth phosphate process

- The reduction-oxidation process

- The PUREX process

- The tributyl phosphate process.

The bismuth phosphate, reduction-oxidation, and PUREX P1 ant processes were specifically designed for plutonium recovery. The initial bismuth phosphate chemical separations process produced large volumes of dilute, low-heat waste. The tributyl phosphate solvent extraction process was designed for the recovery of relatively large amounts of uranium that remained in the bismuth phosphate process waste. The bismuth phosphate process was superseded by the reduction-oxidation process, which was superseded by the PUREX process.

The reduction-oxidation and PUREX processes recovered the uranium and neptunium as well as the plutonium from the irradiated reactor fuel. The PUREX process used solvent extraction with tributyl phosphate to separate uranium and plutonium. Chemical removal of the fuel cladding before extraction produced decladding waste with high concentrations of aluminum and zirconium. High-heat-producing isotopes in the waste were separated from the fuel-reprocessing waste by a modified B Plant waste fractionation process. The strontium was separated by an extraction process using complexing agents (e.g., ethylene-diaminetetraacetic acid, n-hydroxyethylethylenediaminetetraacetic acid, citrate) to prevent transition metal extraction. The cesium was purified by ion exchange. These isotopes (cesium and strontium) wers converted to fluoride and chloride salts and encapsulated in the Waste Encapsulation and Storage Facility. Sodium hydroxide or sodium carbonate was 
added to the waste before transfer to the SSTs to create an alkaline solution and to minimize tank corrosion (RL 1989b). The processing of irradiated fuels produced waste that included most of the fission products and comparatively small quantities of uranium, plutonium, and other actinides (WHC 1990h).

Smaller volumes of waste also were added to the SSTs from research and development programs, facility and equipment decontamination, laboratory activities, and the PFP (RL 1989b).

Waste components in the SSTs have settled, stratified, and segregated. The tanks contain a mixture of nonradioactive and radioactive chemicals produced during the various chemical processes. Therefore, the determination of the actual composition of each tank of waste is complex.

Addition of new waste into the SSTs was terminated in November 1980. Water occasionally is added to certain tanks if necessary for evaporative cooling purposes. This water evaporates and does not add to the waste volume.

\subsubsection{Characterization} sludge.

The SSTs contain radioactive mixed waste that is solid, liquid, and

This section discusses the available waste characterization information. Information based on process knowledge and sample analys is is provided along with the waste designations and their bases, the uncertainty related to the designation, and the schedule for further analysis.

3.3.2.1 Process Knowledge. The SSTs contain irradiated fuel reprocessing waste from separation plants. The tanks received waste from five chemical process activities: the bismuth phosphate, reduction-oxidation, PUREX, and tributyl phosphate processes, and B Plant waste fractionation.

The SSTs contain approximately 139,500 cubic meters of waste as radionuclides and dangerous nonradioactive chemicals. The distribution of the three waste forms (sludge, salt cake, and supernatant) in these tanks is illustrated in Figure 3-4 (WHC 1993e). The salt cake and sludge contain interstitial liquid. The bulk of this liquid, approximately 19,000 cubic meters, is contained in salt cake and is being pumped to the DSTs.

The sludge consists of the solids (hydrous metal oxides, iron, and aluminum) precipitated from the neutralization of acid waste before transfer to the SSTS. Sludges vary greatly in their physical properties. Salt cake contains various salts, primarily sodium nitrate, formed by the evaporation of the water from the waste. Damp salt cake is a jelly-like material; dried salt cake is a hard, abrasive, brittle material that may have formed as large single crystals. The salt cake porosity ranges from 10 to $50 \%$. The liquid exists as supernate and interstitial fluid (WHC 1990h).

Additional equipment components also are found in the tanks with the process waste. These include metal measuring tapes, level instrumentation, other contaminated scrap, pump heads and shafts, and diatamaceous earth. other nonrecorded items are likely to be contained in the tanks. 
3.3.2.2 Sample Analyses. Sample analyses are used to evaluate the chemical, physical, and radiological properties of the SST waste and soils that have been contaminated by spills and leaks. This determination will be used to select a disposal alternative that can be executed safely in compliance with RCRA, the State of Washington Hazardous Waste Management Act of 1976, the National Environmental Policy Act of 1969 (NEPA), and the Atomic Energy Act of 1954 regulatory requirements. The waste is extremely varied with respect to radionuclide content and chemical and physical characteristics. This variation among tanks results from the different nuclear fuel processes and the blending, evaporation, and admixture schemes used since 1944.

A remotely operated method for obtaining samples was developed and implemented for sampling of the liquid and soft, solid tank waste. One to four core samples were removed from each of 15 SSTs in FY 1985 and 1986 . Core samples were analyzed by the individual segment removed or as a homogenized sample of all segments retrieved from each core. The detailed waste analysis results are reported in Weiss (1986) and Adams et al. (1986). A new remote operated method for those SSTs with hard saltcake will be deployed in April 1994.

The SST waste primarily is comprised of sodium hydroxide; sodium salts of nitrate, nitrite, carbonate, aluminate, and phosphate; and hydrous oxides of iron and aluminum. A relatively small amount of solvents was added to the SST waste during fuel reprocessing as well as water-soluble complexing agents and carboxylic acids from the B Plant waste fractionation process (RL 1989b). Initial estimates of inventories of nonradioactive chemicals are given in Table 3-6 (RHO 1985).

Twenty-two of the SSTs contain cyanides, introduced as ferrocyanides in a process to precipitate cesium. Approximately $90 \%$ of the ferrocyanide is in 10 of the tanks. Mixtures of ferrocyanide with sodium nitrate or sodium nitrite may undergo explosive reactions when heated to temperatures significantly above current tank storage temperatures. The buildup of hydrogen under the salt cake in 17 of the SSTs has been a concern. The potential for forming flammable or explosive gas mixtures in the tank vapor space or in gas pockets trapped below the surface of the waste must be considered in retrieval operations (RHO 1985). A large effort currently is under way to resolve these concerns.

A complete, long-term program to characterize SST waste is being conducted by the DOE. This program is detailed in Sasaki (1990). Characterization of al1 149 SSTs and 28 DSTs is scheduled to be completed by September 1999 according to Tri-Party Agreement (Ecology et al. 1992) Milestone M-44-00. The concentration of chemical and radionuci ide species of leaked or spilled materials will require future characterization. Recent characterization data for SSTs have been published (WHC 1993b, WHC 1993C) for two tanks.

3.3.2.3 Waste Designation and Basis. The waste in the SSTs is considered ignitable (due to the presence of nitrate), corrosive, and TCLP toxic. The waste currently is assigned waste codes D001 (ignitable), D002 (corrosive), D005 (TCLP toxic barium), D006 (TCLP toxic cadmium), D007 (TCLP toxic chromium), D008 (TCI.P toxic lead), D009 (TCLP toxic mercury), D010 (TCLP toxic selenium), D011 (TCI.P toxic silver), F003 (acetone and hexone) and F005 
(nonspent solvents). These designations are based on process knowledge and limited sample analyses and may change subject to the results of the analysis and characterization of the waste. The waste designations will be reexamined and revised as necessary as the tanks are characterized.

3.3.2.4 Uncertainty of Waste Designation. The confidence in the current waste code designations is low. The confidence will increase once necessary sampling and analysis work is completed.

3.3.2.5 Schedule for Further Characterization. A commitment has been made to accelerate the characterization of the Hanford Site waste tanks, to expedite the resolution of identified tank safety issues, and to identify tanks that may have safety issues. The current goal is to sample and anaiyze all tanks within the next three years.

\subsubsection{Storage}

This section describes the storage unit, provides the volume currently in storage and projected to be added, and assesses the compliance state of the storage unit.

3.3.3.1 Storage Unit and Capacity. Eighty-three of the SSTs are located in the 200 West Area and 66 are in the 200 East Area. The tanks are arranged in 12 tank farms. One hundred thirty-three of the tanks are 22.9 meters in diameter with nominal capacities between 2,000 and 3,800 cubic meters. Sixteen tanks are 6.1 meters in diameter with capacities of 210 cubic meters (WHC 1990C).

3.3.3.2 Amount in Storage. The SST waste consists of 139,500 cubic meters of solids including 25,000 cubic meters of interstitial 1iquid and supernatant. The volume of waste in each tank farm is shown in Figure 3-5 (WHC 1993e). No waste has been added to the tanks since November 1980 or will be added in the future.

3.3.3.3 Storage Compliance Assessment. The SSTs will be closed in accordance with schedules negotiated in the Tri-Party Agreement (Ecology et a1. 1992). The SSTs were reviewed for compliance with interim status dangerous waste regulations in accordance with Milestone M-21-00. Compliance action schedules and actions for limited compliance with the interim status requirements during the closure are being negotiated.

\subsubsection{Treatment}

This section discusses the current and proposed waste treatment processes.

3.3.4.1 Current Treatment. Ninety-nine of the SSTs have undergone interim stabilization by removal of pumpable liquid. The remaining tanks will undergo interim stabilization operations before disposal. An interim groundwater monitoring program has been established to comply with the interim status dangerous waste requirements found in WAC 173-303 and 40 CFR 265. 
Sixty of the 22.9-meter-diameter SSTs and seven of the 6.1-meter-diameter SSTs (WHC 1990C) are assumed to be past leakers. Unique requirements for waste retrieval from these SSTs have not been identified.

\subsubsection{Proposed and Alternative Treatment. The waste in the SSTs will} undergo retrieval and disposal per the latest planning base. Although the selection of the specific alternative will be documented through the NEPA process, the Tri-Party Agreement specifies that SST waste will be treated and disposed by means of the DST pretreatment and disposal facilities. Closure options, which will identify the level of retrieval necessary, will be documented in a comprehensive tank waste remediation system supplemental environmental impact statement, planned to be prepared.

Waste treated in or retrieved from the SSTs will remain subject to the LDRs unless the following criteria are met:

- Hazardous waste listings applicable to the waste must be identified, and the waste must be desisted in accordance with regulatory requirements

- The treated waste must not exhibit a hazardous waste characteristic (corrosivity, ignitability, reactivity, or TCLP toxicity)

- Treated waste must meet the treatment standards specified by 40 CFR 268.

Waste that meets these requirements would stil11 be subject to the state RCRA program unless the waste does not exhibit any of the dangerous waste criteria for toxicity, persistence, or carcinogenicity of WAC 173-303-101 through -103.

A Tank Waste Technical Options Report was completed in 1992 (WHC 1992c) that presents a number of alternatives for remediating the SSTs and DSTs at Hanford.

3.3.4.3 Accelerated Treatment. Treatment of SST waste is on a schedule based primarily upon Tri-Party Agreement Milestones M-41-00 (interim stabilization) and $M-45-00$ (retrieval technology and closure). (Refer to Figure 2-1 for details.) Because of budget limitations, accelerating treatment beyond these milestone dates is not realistic.

\subsubsection{Waste Reduction}

A waste evaporation program was initiated in 1965 to reduce the volume of liquid waste that potentially could leak and contaminate the soil surrounding the tanks. The supernatant liquids were extracted from the SSTs, evaporated to a slurry, and replaced in the tanks for storage. In 1974 two evaporators were instalied and used to evaporate approximately 510,000 cubic meters of water to date. Further efforts to reduce the potential for leakage include the transfer of waste materials from the SSTs to DSTs. During 1993, portions of tanks BX-110 and -111 were transferred to DSTs. 


\subsubsection{Variances, Exemptions, Time Extensions}

The SST waste consists of radioactive waste mixed with dangerous waste constituents. The SST waste is not subject to LDRs until it is removed from the tanks because it was all generated and $\mathrm{placed}$ in storage before promulgation of LDR regulations.

The Tri-Party Agreement (Ecology et al. 1992) provides for development of treatment and disposal units for the SST waste as follows:

- Complete SST interim stabilization by September 2000

- Develop SST waste retrieval technology and complete scale-model testing by September 1994

- Initiate full-scale tank demonstration of SST waste retrieval technology by October 1997

- Initiate full-scale farm closure demonstration project by December 2003

- Complete closure of all 149 SSTs by September 2024.

If additional variances, exemptions, or time extensions are required as a result of delays in the development of treatment, storage, or disposal capacity, they will be applied for in accordance with the procedures detailed in the Tri-Party Agreement or regulations.

Information on SST waste is provided because of the applicability of LDRS to the waste upon retrieval and because of the need to consider SST waste in establishing treatment capacity requirements for tank waste.

\subsection{2-A EVAPORATOR PROCESS CONDENSATE}

The 242-A Evaporator concentrates the low-level liquid waste that is stored in underground DSTs. The tanks store low-heat-generating waste that contains relatively small amounts of fission products.

The 242-A Evaporator concentrates liquid waste by evaporation. This process reduces the tank waste volume and, hence, the number of DSTs required for storage. The 242-A Evaporator started operating in September 1977; ongoing upgrades will extend its useful life through the year 2000.

In the past (before 1989), the process condensate was routed to retention basins, analyzed for radionuclides and ammonia, and discharged to a crib. In April 1989, dangerous waste and high concentrations of ammonia were detected in the process condensate and discharge to the crib was discontinued. The 242-A Evaporator currently is not operating and is expected to restart in early calendar year 1994. At this time, the process condensate will be discharged to the LERF and ultimately treated for disposal at the 200 Areas Effluent Treatment Facility. 
DOE/RL-94-21

\subsubsection{Generation}

The 242-A Evaporator concentrates 1iquid LLW by evaporation. The evaporation process also separates a large part of the organic constituents and water from the inorganic constituents and radionuclides.

The 242-A Evaporator receives a mixture of waste from DST 241-AW-102 (the evaporator feed tank). This feed tank receives dilute wastes from other DSTs after the waste has been characterized to determine the suitability of the waste for evaporation. A simplified schematic of 242-A Evaporator process operaticrs is shown in Figure 3-6.

The 242-A Evaporator heats the feed, at reduced pressure, and evaporates off some of the water and volatile organic constituents from the slurry. The vapor fraction and slurry fraction are then processed separately. The vapor fraction is condensed, filtered, and discharged to the LERF as process condensate. If the process condensate does not meet discharge limits, it is diverted back to the 242-A Evaporator feed tank for reprocessing. The remaining slurry is recirculated. When the slurry is sufficiently concentrated, it is pumped to underground storage in DSTs.

Upon restart, the 242-A Evaporator will generate up to 17 million liters of process condensate per campaign until the LERF (Section 3.4.3) is full. Three campaigns are scheduled through mid-1995, which are expected to fill the LERF to capacity. The 242-A Evaporator will then be shut down. The 242-A Evaporator will restart when the 200 East Area Effiuent Treatment Facility becomes operational.

\subsubsection{Characterization}

The process condensate is a liquid LLW consisting of the condensed vapor fraction from the evaporation process and raw water. The process condensate is designated a dangerous waste because of toxicity (WTO2) and the potential presence of spent halogenated and nonhalogenated solvents, such as $1,1,1$-trichlorethane, acetone, and methyl isobutyl ketone (hexone) (F001 through F005).

3.4.2.1 Process Knowledge. The 242-A Evaporator receives 1iquid waste from DSTs that originated from most of the Hanford Site waste generators and processes. This waste is processed through the 242-A Evaporator in different batches according to their classification by total organic carbon content, TRU content, and effects on the evaporator process.

Dilute complexed waste is received from processing operations at B Plant. This waste contains high amounts of total organics and complexing agents.

Dilute noncomplexed waste is a mixture of $T$ Plant and S P1 ant waste, PFP supernate, salt well liquids, 300 and 400 Area waste (including fuel fabrication waste), PUREX PI ant neutralized decladding waste supernate, salt wel1 liquids (Section 3.3), and ammonia scrubber waste (Section 3.6)

(WHC 1990j). 
3.4.2.2 Sample Analyses. Process condensate was sampled for characterization from August 1985 to March 1989 during the processing of a variety of evaporator feeds. The average concentration of each analyte detected is shown in Table 3-8 (WHC 1990j).

3.4.2.3 Waste Designation and Basis. The process condensate is designated a dangerous waste because it is derived from waste that may contain the spent halogenated and nonhalogenated solvents 1,1,1-trichloromethane, methylene chloride, acetone, methyl isobutyl ketone, cresylic acid, and methyl ethyl ketone. These constituents together comprise the waste codes F001 through F005.

Additionally, the process condensate is designated a State only dangerous waste because of toxicity (WT02), because of the ammonia concentration. Forty-seven substances potentialiy present in the process condensate were determined to have toxic categories associated with them. The contribution of each substance to the percent equivalent concentration was calculated in accordance with WAC 173-303-101. The resulting equivalent concentration sum is $10 \%$ higher than the 1 imit of $0.001 \%$; therefore, the process condensate is a State toxic dangerous waste. The dominant contributor to the equivalent concentration sum is ammonia.

3.4.2.4 Uncertainty of Waste Designation. The current designations are considered accurate.

3.4.2.5 Schedule for Further Characterization. The process condensate will be characterized after treatment (Section 3.4.4) to confirm that it is no. longer designated dangerous (waste codes no longer applicable).

\subsubsection{Storage}

The 242-A Evaporator currently is not operating and is being modernized. Process condensate, therefore, is not being generated and no process condensate currentiy is in storage. On restart of the 242-A Evaporator, process condensate will be stored at the LERF until a treatment system is operational. The LERF can hold about 49 million liters of process condensate, which is the volume projected to be generated within the first six to twelve months after start-up.

The LERF consists of surface impoundment storage units that will comply with interim status design and operation requirements. A Part B permit application was prepared and submitted in accordance with Tri-Party Agreement (Ecology et al. 1992) Milestone M-20-47 detailing the compliance of the LERF with RCRA final status design and operation standards. Tri-Party Agreement Milestone M-26-04 requires that all hazardous waste residues that do not meet LDR treatment standards be removed from the LERF by June 1995. A change request is being prepared to change this milestone to support the 200 Areas Effluent Treatment Facility start-up date. 
DOE/RL-94-21

\subsubsection{Treatment}

3.4.4.1 Planned Treatment. Planned treatment of the process condensate stored at the LERF is as follows. The 200 Areas Effluent Treatment Facility will treat process condensate and prepare the waste for disposal. The current draft process flow diagram is as described below.

- The first step is to adjust the $\mathrm{pH}$ of the waste stream with sulfuric acid to a $\mathrm{pH}$ of about 6 within a 100,000-gallon surge tank using a recirculation pump.

- Next, suspended particles are filtered by a roughing filter.

- Then an organic destruction unit uses hydrogen peroxide and/or ozone with ultraviolet light to degrade organic compounds into carbon dioxide and water.

- The $\mathrm{pH}$ of the waste stream is then lowered to $\&$ by the addition of sulfuric acid. This adjustment insures all ammonia is converted into its ammonium salt, thereby conditioning the ammonia (as a salt) to be removed by reverse osmosis in a subsequent treatment step. Adjustment of the $\mathrm{pH}$ to 4 also converts carbonate and bicarbonate to carbon dioxide for removal by a degasser in a subsequent step.

- Next, a filtration step removes residual particulates down to about $0.5 \mathrm{\mu m}$.

- Following that degasification removes the carbon dioxide generated in previous treatment steps.

- Then reverse osmosis removes aqueous salts (including metal ions, radionuclides, and ammonium sulfate) producing a secondary waste stream that will be further concentrated by an evaporation process in subsequent steps.

- The stream is next treated by ion exchange to remove residual aqueous salts not removed by reverse osmosis.

- The treated stream is neutralized as necessary and sent to verification tanks. A system of three verification tanks hold the treated effluent for sampling before discharge (current plans call for discharge to a state-approved land disposal structure). A recycle loop is provided should verification analyses show that a rework is required to meet permit conditions.

- Secondary waste (primarily produced from the reverse osmosis step, regeneration wastes from the ion exchange step, and blow-down from the two filtration steps) is sent to an evaporation process consisting of a mechanical vapor recompression evaporator and a. thin-film dryer. Feed to the evaporation process will be routinely analyzed to determine the nature of the dry secondary waste product. If the dried secondary waste product is a hazardous or dangerous 
waste, it will be sent to the CWC and treated at the WRAP Facility. If it is not hazardous or dangerous, the drums will be disposed of at the low-level burial grounds.

The treatment facility is scheduled to begin operations in June 1995 in accordance with Tri-Party Agreement Milestone M-17-14.

3.4.4.2 Treatment Alternatives and Accelerated Treatment. The treatment method for 242-A Process Condensate has been established. Startup of the effluent treatment facility is a priority and accelerating treatment is not realistic.

\subsubsection{Waste Reduction}

Planned treatment of the process condensate will result in a nondangerous liquid stream acceptable for discharge to the ground and a solid waste form acceptable for storage at the Central Waste Conplex.

The treatment unit will reduce each 245 cubic meters of process condensate to one 0.21 -cubic-meter drum of solid waste; this is a waste reduction factor of 1,200 . This reduction is based on two assumptions: the average concentration of ammonium in process condensate is $410 \mathrm{ppm}$ and the waste product in the drums is $100 \%$ ammonium sulfate.

\subsubsection{Variances, Exemptions, Time Extensions}

The 242-A Evaporator process condensate is a LLW mixed waste that is LDR because it contains solvent list ( 40 CFR 268.30 ) constituents. Currently process condensate is not generated because the 242-A Evaporator is shut down for modifications. Additional process condensate will be generated when the 242-A Evaporator is restarted.

The national capacity variance for Third-Third Waste (55 FR 22520) provided for a 2-year national capacity variance from the LDR for Third-Third mixed waste expiring on May 8, 1992. This variance allowed continued storage of these wastes. A Federal Register notice dated May 26, 1992, stated that EPA could not grant a case-by-case extension for Third-Third mixed waste because of questions regarding the DOE's demonstration that they have entered into binding contractual commitments to construct or otherwise provide treatment capability. A final decision has yet to be made. This extension would allow DOE to store LDR mixed wastes until May 8, 1993, and this date could be extended for up to an additional year. The Tri-Party Agreement will allow continued storage of these wastes in accordance with the schedules in the Tri-Party Agreement. The Federal Facility Compliance Act of 1992 also allows DOE facilities to store their wastes for 3 years if compliance with the Act's provisions for providing waste inventory and treatment plans are met. 
The Tri-Party agreement requires treatment and disposal capacity for this waste to be developed on the following schedule:

- Cessation of discharge of process condensate to the LERF by December 1994. (This may be affected by a change request that has been drafted to change the date to support the 200 Areas Effluent Treatment Facility start-up date of June 1995.)

- Removal of al1 dangerous waste residues from the LERF by June 1995. (This may be affected by a change request that has been drafted to change the date to support the 200 Areas Effluent Treatment Facility start-up date of June 1995.)

Future process condensate generated will be discharged to and stored in the LERF until the Effluent Treatment Facility is constructed and ope ational. The 200 Areas Effluent Treatment Facility will treat process condensate to meet the LDRs. Additionally, a petition is being prepared to delist the efiluent from the 200 Areas Effluent Treatment Facility to allow land disposal of the treated effluent.

Part B Permit applications will or have been submitted for the 242-A Evaporator (completed June 1991), the LERF (completed June 1991), and the 200 Areas Effiuent Treatment Facility (due in August 1993). The delisting petition for the 200 Areas Effluent Treatment Facility was submitted in October 1992.

If additional variances, exemptions, or time extensions are required because of delays in the development of treatment, storage, or disposal capacity, they will be applied for in accordance with the procedures detailed in the Tri-Party Agreement or regulations.

\subsection{SODIUM STORAGE FACILITY WASTE}

The 4843 Sodium Storage Facility received radioactive and nonradioactive alkal i metal waste from Hanford Site generators. The predominant generator of alkali metal waste was the FFTF.

Most of the waste received at the 4843 Sodium Storage Facility consisted of alkali metals and retired equipment from liquid sodium processes. The bulk of material presently in storage is sodium derived from normal FFTF operations and a pump leak at the FFTF.

The waste stored in the 4843 Sodium Storage Facility currently is untreated. The nonradioactive material will be sent offsite for treatment while the radioactive portions would be treated and disposed of onsite with methods to be determined. This facility is scheduled for closure and a closure plan has been prepared. Waste in storage now will be transferred to the CWC where it will be stored until future processing and disposal

facilities are made available. 


\subsubsection{Generation}

The FFTF is an experimental reactor that uses liquid sodium in the primary coolant loop. One cubic meter of sodium and 0.5 cubic meter of structural and other equipment were generated by a pump leak at the FFTF.

Seven drums of waste radioactive sodium have been generated at the FFTF as a result of normal operations during the past 10 years. The rate of future waste production is anticipated to decrease because of a modification in the FFTF procedures that permits recycling of some of this material. The FFTF facility has received a shutdown directive. This eliminates waste generated due to operations.

The 4843 Sodium Storage Facility became operational in September 1987 to receive radioactive and nonradioactive alkali metal waste from Hanford Site generators. Most of the waste received at the 4843 Sodium Storage Facility consists of spill residue and retired equipment from 1 iquid sodium processes at the FFTF. The 4843 Sodium Storage Facility no longer receives waste for storage.

\subsubsection{Characterization}

This section discusses the available waste characterization information. Information based on process knowledge and sample analys is is provided along with the waste designation and basis. The uncertainty related to the designation and the schedule for further analysis also are discussed.

3.5.2.1 Process Knowledge. All material in the 4843 Sodium Storage Facility is solid, nonradioactive, or radioactive LLW. All of the waste sodium in the storage unit has been generated at the FFTF from normal operations, a pump leak, and miscellaneous experimental apparatus.

3.5.2.2 Sample Analyses. The waste in the 4843 Sodium Storage Facility is characterized based on process knowledge. No further analysis has been considered at this time.

3.5.2.3 Waste Designation and Basis. The alkali metal waste received for storage at the $\mathbf{4 8 4 3}$ Sodium Storage Facility is characterized as ignitable (D001), corrosive (D002), reactive (D003), and toxic (WTO1 and WTO2).

3.5.2.4 Uncertainty of Waste Designation. The waste characterization certainty is considered high, based on derivation of the waste from sodium cooling loops and experimental apparatus.

3.5.2.5 Schedule for Further Characterization. No further characterization of the waste stored in the 4843 Sodium Storage Facility is anticipated.

During future treatment the residues will be analyzed chemically to verify completeness of treatment and to designate the waste for proper disposal. 


\subsubsection{Storage}

This section describes the storage unit, provides the amount in storage, and assesses the compliance status of the unit.

3.5.3.1 Description of Storage Unit and Capacity. The 4843 Sodium Storage Facility waste storage unit is located in the northwest corner of the 400 Area of the Hanford Site. There are no other buildings in the immediate vicinity of the 4843 Sodium Storage Facility. The gravel area surrounding the building is clear of combustibles for several hundred meters. The building is 12 meters long, 12 meters wide, and 6 meters high. The building has an all-steei structural frame and sides and a gable roof, all of which are insulated with fiberglass batting. The floor is a concrete slab. Building access is through two large rollup doors in the east and west ends and through personnel doors in the southeast and northwest corners.

The 4843 Sodium Storage Facility is used to store radioactive and nonradioactive alkali metal waste, including sodium, lithium, and a sodium/potassium mixture, which has been generated at the FFTF and other operations at the Hanford Site that use alkali metals. (Waste has become radioactive through use in the sodium-cooled reactor.) Waste is segregated within the building depending on whether the alkali metal is radioactive or nonradioactive. Radioactive alkali metal waste is stored in 0.21 -cubic meter drums, various piping sections, and "hot-traps." Nonradioactive alkali metal waste is stored in the southern half of the building. The radioactive and nonradioactive storage areas are separated by a radiation boundary rope divider.

The 4843 Sodium Storage Facility only accepted solid alkali metal waste properly packaged in U.S. Department of Transportation-specified containers. To keep the reactive alkali metal waste stable, these containers are flushed with inert gas (argon) and sealed to provide a nonreactive atmosphere.

The estimated capacity of the 4843 Sodium Storage Facility is 84,000 kilograms of alkali metal (RL 1989a).

3.5.3.2 Amount in Storage. The current inventory of the 4843 Sodium Storage Facility includes 8.5 cubic meters of radioactive waste contaminated with 872 kilograms of sodium, and 0.02 cubic meters of nonradioactive waste contaminated with 0.05 kilograms of lithium.

3.5.3.3 Storage Compliance Assessment. The 4843 Sodium Storage Facility was reviewed for compliance with interim status dângerous waste regulations in accordance with Tri-Party Agreement (Ecology et al. 1992) Milestone M-21-00. No areas of noncompliance with interim status requirements were noted other than the since-completed development of a waste analysis $p 1$ an and a contingency plan. The facility is now scheduled for closure. The closure plan has been prepared and it is anticipated that closure will be completed in FY 1993.

\subsubsection{Treatment}

This section discusses the current and proposed waste treatment. 
3.5.4.1 Current Treatment. The 4843 Sodium Storage Facility is a storage unit. The waste stored in this unit currently is not being treated.

3.5.4.2 Proposed Treatment. Original plans called for this facility to be fully permitted as a RCRA storage unit. A Part B Permit application was prepared and submitted for internal review in March 1991. Subsequently a decision was made to close the 4843 Sodium Storage Facility. The closure plan has been prepared and transmitted to Ecology in June 1991. According to the provision of these plans, the nonradioactive alkali metal waste will be sent offsite to an approved facility for treatment and disposal while the radiositive alkali waste will be transported to the CWC for storage until appropriate treatment and disposal systems are available. All but one container of nonradioactive waste already has been shipped offsite. It is anticipated that the closure will be completed during FY 1994. A considered method for treatment involves the conversion of sodium to sodium hydroxide and then to sodium carbonate. The sodium carbonate would be designated and disposed of in accordance with applicable regulations. Further planning for treatment alternatives or accelerated treatment will be handled as with other CWC Waste (Section 3.13).

\subsubsection{Haste Reduction}

The 4843 Sodium Storage Facility is a storage unit that received alkali metal waste generated on the Hanford Site. Waste generated at the 4843 Sodium Storage Facility is managed to ensure that the quantity and toxicity are minimized. No waste is anticipated to be generated after 1993.

The 4843 Sodium Storage Facility has an operating procedure for the disposal of waste generated at the 4843 Sodium Storage Facility Waste that includes proper responses for cleanup after dangerous waste spills. The response to dangerous waste spills is aimed at minimizing liquid and material used during cleanup. Conversion to carbonate, if this is the chosen treatment method, would remove the entire inventory of elemental sodium waste (see Section 3.5.4.2).

\subsubsection{Variances, Exemptions, Time Extensions}

The national capacity variance for Third-Third Waste (55 FR 22520) provided for a 2-year national capacity variance from the LDR for Third-Third mixed waste expiring on May 8, 1992. This variance allowed continued storage of these wastes. A Federal Register notice dated May 26, 1992, stated that EPA could not grant a case-by-case extension for Third-Third mixed waste because of questions regarding the DOE's demonstration that they have entered into binding contractual commitments to construct or otherwise provide treatment capability. A final decision has yet to be made. This extension would allow DOE to store LDR mixed wastes until May 8, 1993, and this date could be extended for up to an additional year. The Tri-Party Agreement will allow continued storage of these wastes in accordance with the schedules in the Tri-Party Agreement. The Federal Facility Compliance Act of 1992 also allows DOE facilities to store their wastes for 3 years if compliance with the Act's provisions for providing waste inventory and treatment plans are met. 


\subsection{PUREX AMMONIA SCRUBBER WASTE}

The ammonia scrubber waste is a mixed LLW liquid effluent that was generated by the PUREX ?lant. During PUREX PIant operations, approximately 7,600 cubic meters of ammonia scrubber feed were generated per year. The ammonia scrubber feed is designated as toxic (WTO1) because of the concentration of ammonia in some operating modes. The most recent fraction of ammonia scrubber feed was treated with sodium hydroxide in preparation for tank storage. The treated ammonia scrubber waste is designated as corrosive (D002) as well as toxic (WTO1) and is a LDR waste. No additional ammonia scrubber waste has bern yenerated since December 1989 and none will be generated in the future.

\subsubsection{Generation}

The PUREX Plant received irradiated zirconium clad fuel from $N$ Reactor, removed the cladding from the fuel, and dissolved the fuel in nitric acid. The dissolved fuel was processed through several solvent extraction steps to separate the plutonium, uranium, and neptunium from the fission products contained in the fuel. The PUREX ammonia scrubber feed was generated when water was sprayed to adsorb ammonia gas generated by the decladding and metathesis reactions from the dissolver offgas stream.

In the past, the ammonia scrubber feed was boiled in a concentrator to separate the bulk of the water from the entrained fission products. The condensed water vapors were disposed of to a crib. The remaining ammonia scrubber waste was treated to comply with DST storage specifications and transferred to DSTs as shown in Figure 3-7.

In late 1987, it was determined that the ammonium hydroxide concentration in the ammonia scrubber condensate sometimes exceeded $1 \%$, making it a dangerous (toxic) waste as designated by state regulations; therefore it is not appropriate for discharge to the crib. An interim process was established in which ammonia scrubber feed no longer was concentrated for discharge, but was treated for tank storage and transferred as ammonia scrubber waste to underground storage tanks. The treatment consisted of adding sodium hydroxide to adjust the $\mathrm{pH}$ to greater than 12 and adding sodium nitrite to minimize tank corrosion.

Approximately 15 cubic meters of ammonia scrubber feed was generated per metric ton of uranium processed. The amount of ammonia scrubber waste generated by month for 1988 is shown in Figure 3-8. No ammonia scrubber waste has been generated since December 1989 and none will be generated in the future.

\subsubsection{Characterization}

This section discusses the available waste characterization information. Information based on process knowledge and sample analyses is provided along with the waste designation and its basis, the uncertainty related to the designation, and the schedule for further analysis. 
3.6.2.1 Process Knowledge. The ammonia scrubber feed waste stream comprises water, ammonium hydroxide, dissolved ammonia, trace amounts of radionuclides, and fiuoride and nitrate ions from the ammonium fluoride-ammonium nitrate solution used in the dissolver. The $\mathrm{pH}$ of the ammonia scrubber feed stream before treatment for tank storage is between 8 and 10 . In the past, the ammonia scrubber waste was similar in composition to the ammonia scrubber feed except that $99 \%$ of the ammonia present in the ammonia scrubber feed was removed by volatilization during waste concentration and was discarded into the ammonia offgas system or with the ammonia scrubber condensate waste stream.

3.6.2.2 Sample Analyses. The management of the PUREX ammonia scrubber waste can be divided as follows:

- The ammonia scrubber feed produced before late 1987 , most of which was evaporated, condensed, and discharged to cribs as ammonia scrubber discharge (Figure 3-7)

- The total ammonia scrubber feed generated after crib closure in 1987, which was then treated and sen'i as ammonia scrubber waste to DSTs for storage.

The ammonta scrubber discharge was sampled randomly four times over a 23-month period during routine operation, once in 1985 and three times in 1987. The number of chemical analyses detected was 12, although not every analyte was detected at each sampling time. Table 3-9 summarizes the analytical results (WHC 1990f).

The ammonia scrubber feed stored in the DSTs is treated with sodium hydroxide and sodium nitrite. Availabie analytical data for this stream are shown in Table 3-10.

3.6.2.3 Waste Designation and Basis. Both the historical and PUREX ammonia scrubber waste streams are toxic liquid, noncombustible LLWs classified as wastewaters.

The ammonia scrubber feed stream treated and sent to tank storage is toxic because of the concentration of ammonia. Pursuant to WAC 173-303-070, its designation is WTO1. Treating the ammonia scrubber feed with sodium hydroxide, to raise the $\mathrm{pH}$ above 12, occasionally renders the resulting ammonia scrubber waste corrosive (D002) as well and creates land disposal restricted waste (WHC 1990f).

3.6.2.4 Uncertainty of Waste Designation. Waste designations for the ammonia scrubber waste sent to tank storage are based on sample analyses. Actual sample results show that the ammonia concentration exceeds 1 weight percent during the first few hours of the decladding reaction. The dangerous waste designation due to ammonia for these streams is only a result of exceeding the 1 weight percent 1 imit for a few hours during each decladding reaction. The average concentration for ammonia in this waste is less than $0.1 M$ as shown in Table 3-9. 
Based on the chemicals added to the ammonia scrubber waste that was sent to DSTs and on sample analyses, the ammonia scrubber waste is a toxic (WTO1) and may be a corrosive (D002) LDR waste.

3.6.2.5 Schedule for Further Characterization. The ammonia scrubber waste currently stored in tanks will be characterized before planned treatment and disposal of the tank contents. Underlying hazardous constituents defined by the emergency third-third rule decision (58 FR 29860) reasonably expected to be present at that time will be quantitized. The tank contents will be concentrated at the 242-A Evaporator to reduce the volume of waste requiring vitrification and disposal. The identification of additional waste characterization tasks wili be negotiated among Ecology, EPA, and DOE (WHC 1990f).

\subsubsection{Storage}

This section provides the volume currently in storage and assesses the enmpliance state of the storage unit.

3.6.3.1 Storage Unit and Capacity. The PUREX ammonia scrubber waste is stored in underground DSTs in the 200 East Area of the Hanford Site. The tank farms have 28 4,300 cubic meters tanks, of which 26 store nonaging waste. The total contents of the DSTs are addressed as a single waste stream in Section 3.1.

3.6.3.2 Assount in Storage. The amount of DST waste in storage contributed by ammonia scrubber waste is 5,900 cubic meters. The volume of waste requiring disposal will decrease when the waste is evaporated before disposal. The capacity of the tank farms for continued waste storage is discussed in Section 3.1 .

3.6.3.3 Storage Compliance Assessment. The PUREX ammonia scrubber waste is stored in the DSTs. The DSTs were reviewed for compliance with interim status dangerous waste regulations in accordance with Tri-Party Agreement (Ecology et al. 1992) Milestone M-21-00. The results of the compliance assessment are provided in Section 3.1.3.3.

\subsubsection{Treatment}

The ammonia scrubber waste has been treated for storage by adding sodium hydroxide and sodium nitrite to control tank corrosivity. The stream in the DSTs will be concentrated at the 242-A Evaporator. Refer to DST treatment plans for future treatment information.

\subsubsection{Waste Reduction}

Change in operational status has el iminated the ammonia scrubber waste; therefore, waste reduction is not applicable. 


\subsubsection{Variances, Exemptions, Time Extensions}

The national capacity variance for Third-Third Waste (55 FR 22520) provided for a 2-year national capacity variance from the LDR for Third-Third mixed waste expiring on May 8, 1992. This variance allowed continued storage of these wastes. A Federal Register notice dated May 26, 1992, stated that EPA could not grant a case-by-case extension for Third-Third mixed waste because of questions regarding the DOE's demonstration that they have entered into binding contractual commitments to construct or otherwise provide treatment capability. A final decision has yet to be made. This extension would allow DOE to store LDR mixed wastes until May 8, 1993, and this cate could be extended for up to an additional year. The Tri-Party Agreement will allow continued storage of these wastes in accordance with the schedules in the Tri-Party Agreement. The Federal Facility Compliance Act of 1992 also allows DOE facilities to store their wastes for 3 years if compliance with the Act's provisions for providing waste inventory and treatment plans are met.

If additional variances, exemptions, or extensions of time are required because of delays in the development of treatment, storage, or disposal capacity, they will be applied for in accordance with the procedures detailed in the Tri-Party Agreement or reguiations.

\subsection{PUREX PROCESS CONDENSATE}

The PUREX process condensate was a mixed LLW liquid effluent generated by the PUREX Plant. As of April 1, 1990, approximately 4,800 cubic meters of PUREX process condensate have been generated and are stored in DSTs. No additional PUREX process condensate has been generated since March 1990 and none is expected to be generated in the future.

The PUREX process condensate is distilled water with a nitric acid content that can exceed $0.01 \mathrm{M}(\mathrm{pH} 2)$. Additionally, the stream contains traces of various radionuclides. Until 1987, the PUREX process condensate stream was discharged directly to a crib. After closure of the crib and to prevent corrosive (pH less than 2) waste from being discharged into the new crib, potassium hydroxide was added and the stream was routed through a tank with calcium carbonate (1imestone) before being discharged. In early 1989 the stream was temporarily rerouted to DSTs during a reevaluation of its dangerous waste designation. The PUREX process condensate transferred to DSTs was designated corrosive (D002).

\subsubsection{Generation}

The PUREX Plant received irradiated zirconium clad fuel from $N$ Reactor, removed the cladding from the fuel, and dissolved the fuel in nitric acid. The dissolved fuel was processed through several solvent extraction steps to separate the plutonium, uranium, and neptunium from the fission products contained in the fuel.

The PUREX process condensate stream was generated by condensing vapors from uranium/nitric acid concentration and recycle processes within PUREX. This condensate contains trace quantities of nitric acid. Before 1987, the 
condensate was monitored for radioactivity and discharged to a crib. In 1987 , the PUREX process condensate system was upgraded to include a potassium hydroxide neutralization system and a calcium carbonate neutralization bed to neutralize the traces of nitric acid in the PUREX process condensate before discharge to a second crib (Figure 3-9).

In 1989, while reevaluating the designation of the PUREX process condensate stream to ensure that no improperiy designated waste was being discharged to the environment, the PUREX process condensate waste stream was treated to meet tank storage specifications and to be transferred to the DSTs. since March 1990, no PUREX process condensate has been generated.

Approximately 44 cubic meters of PUREX process condensate was generated per metric ton of uranium processed.

\subsubsection{Characterization}

This section discusses the available waste characterization information. The information is gathered from process knowledge and sample analyses data. Preliminary waste designation and basis, the uncertainty related to the designation, and the schedule for further analysis is provided.

3.7.2.1 Process Knowledge. Before 1986, the traces of nitric acid that distilled over with the PUREX process condensate were not neutralized before discharging that stream to a crib. After 1986, a neutralization system was installed that included controlled addition of potassium hydroxide to the PUREX process condensate, a pH polishing tank containing calcium carbonate (crushed limestone), and $\mathrm{pH}$ monitoring instrumentation (Figure 3-9)

(WHC 1990g).

3.7.2.2 Sample Analysis. During PUREX operations, PUREX process condensate is sampled as indicated below.

- Pre-1989--PUREX process condensate was sampled with a weekly composite sampler system. Samples were collected in a tank over a 1-week period and analyzed for key radionuclides, $\mathrm{pH}$, organics, and uranium.

- 1989-1990 Stabilization Run--PUREX process condensate was batch sampled for $\mathrm{pH}, \mathrm{NO}_{2}$, and uranium and sent to DSTs.

Before 1989, samples of the PUREX process condensate stream going to the crib were analyzed. The PUREX process condensate was randomly sampled eight times over a 24-month period during routine operations, once in 1985, twice in 1986, and five times in 1987 . The number of chemical constituents detected was 46, although not every constituent was detected in each sample.

Table 3-11 summarizes the analytical results (WHC 1990g).

3.7.2.3 Waste Designation and Basis. Before 1987, the PUREX process condensate waste stream was occasionally corrosive ( $\mathrm{pH}$ less than 2) because of the nitric acid present in the PUREX process condensate. Under these conditions, the occasionaliy corrosive stream would have been designated as a corrosive (D002). After the neutralization system was installed in 1987, the 
PUREX process condensate stream was nondangerous. During a reassessment of the designation of the PUREX process condensate waste stream to ensure that no discharge to the environment of improperly designated waste was occurring, the PUREX process condensate was rerouted to DSTs in early 1989. The PUREX process condensate waste stream sent to the OSTs was treated with sodium hydroxide (to adjust $\mathrm{pH}$ to above 12) and sodium nitrite (to control tank corrosivity). This treated waste was designated corrosive $\mathrm{D002}$.

3.7.2.4 Uncertainty of Waste Designation. The PUREX process condensate stream designation is based on process knowledge and sample analyses that are representative of the normal process. There are potential upset conditions and unusual occurrences that could create a corrosive dangerous waste. However, no unusual or abnormal events have occurred that would change the waste designation for the waste sent to DSTs.

3.7.2.5 Scheduie for Further Characterization. No future characterization of PUREX process condensate is planned except as part of the treatment process for DST contents (similar to ammonia scrubber waste, Section 3.6.2.5).

\subsubsection{Storage}

This section discusses the PUREX process condensate waste storage and capacity, identifies stored quantities, and assesses the compliance status of the storage unit.

3.7.3.1 Storage Unit and Capacity. The PUREX process condensate waste is stored in underground DSTs in the 200 East Area of the Hanford Site. These tanks are discussed in Section 3.1.

3.7.3.2 Amount in Storage. As of April 1, 1990, approximately 4,800 cubic meters of PUREX process condensate waste were stored in the DSTs.

3.7.3.3 Storage Compliance Assessment. The previously generated PUREX process condensate is stored in the DSTs. The DSTs were reviewed for compliance with interim status dangerous waste regulations in accordance with Tri-Party Agreement (Ecology et al. 1992) Milestone M-21-00. The results of the compliance assessment are provided in Section 3.1.3.3.

\subsubsection{Treatment}

The PUREX process condensate has been treated for storage by adding sodium hydroxide and sodium nitrite to control tank corrosivity. . Refer to DST treatment plans for future treatment information.

\subsubsection{Waste Reduction}

Waste reduction is not applicable at this time. The PUREX Plant will not be restarted. 


\subsubsection{Variances, Exemptions, Time Extensions}

Previously generated PUREX process condensate waste was restricted from 1 and disposal by the Third-Third Promulgation (55 FR 22520). As a component of the DST contents, PUREX process condensate will be treated and disposed of in accordance with the plans for DST LLW discussed in Chapter 3.0, Section 3.1.6.

The national capacity variance for Third-Third Waste (55 FR 22520) provided for a 2-year national capacity variance from the LDR for Third-Third mixed waste expiring on May 8, 1992. This variance allowed continued storage of these wastes. A Federal Register notice dated May 26, 1992, stated that EPA could not grant a case-by-case extension for Third-Third mixed waste because of questions regarding the DOE's demonstration that they have entered into binding contractual commitments to construct or otherwise provide treatment capability. A final decision has yet to be made. This extension would allow DOE to store LDR mixed wastes until May 8, 1993, and this date could be extended for up to an additional year. The Tri-Party Agreement will allow continued storage of these wastes in accordance with the schedules in the Tri-Party Agreement. The Federal Facility Compliance Act of 1992 also allows DOE facilities to store their wastes for 3 years if compliance with the Act's provisions for providing waste inventory and treatment plans are met.

If additional variances, exemptions, or extensions of time are required because of delays in the development of treatment, storage, or disposal capacity, they will be applied for in accordance with the procedures detailed in the Tri-Party Agreement or regulations.

\subsection{HEXONE WASTE}

One hundred thirty-six cubic meters of liquid mixed LLW, primarily hexone, formerly were stored in two underground tanks near the 202-S Plant in the 200 West Area. The waste was distilled to remove radionuclides and is being incinerated to destroy the hexone. Hexone waste is no longer being generated.

\subsubsection{Generation}

The 202-S Plant used solvent extraction with hexone to separate uranium and piutonium from reactor fuel. The 202-S P1ant operated from 1951 to 1967 (DOE 1987).

The hexone was stored in two underground tanks. Tank 276-S-141 contained 76 cubic meters of hexone that was distilled before storage. Tank 276-5-142 contained 53 cubic meters of mixed solvents and 8 cubic meters of water. The mixed solvents were $65 \%$ hexone, $25 \% \mathrm{~N}$-alkanes (normal paraffin hydrocarbon), and $9 \%$ tributyl phosphate that were added to the tank as spent solvent from a one-time americium extraction campaign at the 202-S Plant. Tank 276-S-142 
also contained 8 cubic meters of water, most of which was added to the tank to flush transfer piping. The tanks also contained about 0.4 cubic meters of sludge, primarily tank corrosion products.

The hexone waste is no longer being generated.

\subsubsection{Characterization}

The hexone (methyl isobutyl ketone) waste is a dangerous 1iquid LLW. The dangerous waste codes for this material are FO03 (hexone), WTO2 (toxic dangerous waste), WCO2 (carcinogenic), and D0O1 (ignitabie). The result of a chemical analysis for each tank (before distillation as well as the residual after distillation) is shown in Table 3-12.

After distillation, there were 16,691 gallons of pure hexone in two tank cars and 12,198 gallons of a mixture of hexone, kerosene, and small amounts of tributyl phosphate (less than 1\%) in two other tank cars. Also in the latter two tank cars were 4,171 gallons of water stored with hexone ( 1 to $2 \%$ ). Between the four cars, there was 0.71 curie of tritium. All of the distilled material was trucked to the Diversified Scientific Services, Inc. incinerator in Kingston, Tennessee. It has been burned except for 2,000 gallons of solvent-saturated water that is scheduled for incineration in March/Apri1 1994.

The distillation tars have a volume of 250 gallons and contain essentially all of the nonvolatile radionuclides. The tar has been analyzed and found to be non-TRU mixed waste.

\subsubsection{Storage}

The waste remaining in the two original storage tanks will be handled and disposed of as part of the tank closure process.

\subsubsection{Treatment}

During 1990, the waste was treated by distillation to remove radionuclides to allow disposal of the bulk of the waste by incineration. The next treatment step was offsite incineration to destroy the hexone. This treatment was nearly complete as this was written and is expected to be finished by April 1994. The last of the hexone was shipped off the Hanford Site in June 1992. A flowsheet summarizing the treatment and disposal of hexone waste is shown in Figure 3-10.

Distillation of the liquid waste produced three primary product streams: the "clean" distillate, the tar-like bottoms in the distillation vessel, and the offgases of the distillation. There also remains in Tanks 276-5-141 and -142 a liquid level of under 50 gallons and approximately 250 gallons of sludge in each tank. 
The tar-1ike bottoms will remain in the distillation vessels, and the vessels have been sealed for disposal. The vessels are 0.9 meters in diameter and 1.9 meters long, with an approximate weight of 860 kilograms. The spent vesse1s are non-TRU and are stored at the CWC for further treatment by the WRAP Facility. Waste minimization was achieved by minimizing the number of vessel changeouts.

The offgasses were vented back through the underground tanks to maximize condensation (minimizing gaseous effiuents and the amount of activated charcoal required for treatment) and treated by high-efficiency filtration and charcoal adsorption and filtration. The charcoal adsorbent becomes a mixed waste. Approximately 270 kilograms of charcoal (six 0.21-cubic meter drums) were used.

The waste remaining in the two original storage tanks will be handled and disposed of as part of the tank closure process.

As with the distillation phase, the treatment by incineration is itself a waste reduction effort because it will eliminate a dangerous waste (the incineration process will reduce the organic distillate to nondangerous carbon dioxide and water).

Accelerated treatment of the bulk of the hexone waste is not applicable; it has already been treated. Any accelerated treatment of the bottoms in the distillation vessels would be provided by the WRAP Facility (Section 3.13.4). The tank closure process will dispose of the waste in the two original storage tanks. Similariy, alternative treatments would be considered as part of the design and operation of the WRAP Facility.

\subsubsection{Waste Reduction}

Distillation has reduced the volume of mixed waste from 136 cubic meters of hexone waste to less than 1.6 cubic meters of tar-filled vessels and 1 cubic meter of charcoal adsorbent. Most of the equipment has been dismantled, packaged, and shipped to the Central Waste Complex for storage before final treatment and disposal. Additional reduction information is located in Section 3.8.4. No true waste minimization efforts are in effect for hexone waste because it is no longer being generated.

\subsubsection{Variances, Exemptions, Time Extensions}

Hexone is a mixed LLW that is restricted from land disposal because it contains solvent list ( 40 CFR 268.30) constituents.

If additional variances, exemptions, or time extensions are required because of delays in the development of treatment, storage, or disposal capacity, they will be applied for in accordance with the procedures detailed in the Tri-Party Agreement. 


\subsection{3-H SOLAR EVAPORATION BASINS WASTE}

The 183-H Solar Evaporation Basins are being closed under RCRA Regulations, WAC 173-303, and Tri-Party Agreement sitewide permit conditions.

The 183-H Solar Evaporation Basins are four concrete basins located in the 100-H Arei. The 183-H Basins were constructed in 1949. Originally there were 16 flocculation and sedimentation basins that were a part of the 183-H Filter Plant. The filter plant provided water treatment, filtering units, and reservoir capacity for the 100-H Reactor process water system. In the spring of 1974, after decontamination, demolition of the 183-H Filter Plant was initiated. The 183-H headhouse, 12 of the flocculation and sedimentation basins, the filter building, and the clearwell pump room were demolished to groundievel and the underground portions were backfilled to groundlevel. The remaining four basins were used from 1973 to 1985 to store and treat liquid chemical waste from 300 Area fuel fabrication plants. The purpose of the 183-H Basins was to provide a means of waste reduction by natural solar evaporation.

The waste stored in the 183-H Basins has undergone sol.ar evaporation. The waste precipitates and the residual liquids have been treated by solidification; sludges have been removed; everything has been packaged in lined 0.21-cubic meter drums, and shipped to the CWC for storage and future processing at the WRAP Facility.

\subsubsection{Generation}

The 183-H Basins were a storage and treatment (evaporation) unit for the liquid chemical waste generated at the 300 Area nuclear fuel fabrication plants. The basins received waste from 1973 through 1985 (RL 1991c).

During the operating life of the 183-H Basins a total of 9,623 cubic meters of routine waste was added to the basins. Table 3-13 presents the quantity of chemical constituents discharged to the basins.

In addition to the routine waste, nonroutine waste periodically was discharged into the 183-H Basins. Nonroutine waste consisted of unused chemicals and spent solutions from miscellaneous processes, development tests, and laboratories. Nonroutine waste fell into three categories: 1isted waste, nonlisted waste that was added directly to the 183- $\mathrm{H}$ Basins, and nonlisted waste that was mixed with the routine waste stream before being transported to the 183-H Basins. Only a small amount of 1 isted nonroutine waste was discharged to the basins. The listed waste quantities were estimated to be 2 kilograms of solid materials and 9 liters of solution. Nonlisted, nonroutine waste discharged directly into the 183-H Basins totaled approximately 50 kilograms of apparently dangerous solid materials, less than 5.8 cubic meters of apparently dangerous liquid waste, and 39 cubic meters of nondesignated waste. Internal "chemical waste disposal permit" records indicate that about 44.30 cubic meters of liquid waste and 700 kilograms of solid waste was mixed with routine waste before being discharged into the 183-H Basins (RL 1991c). 
The quantity of waste removed from the basins and now stored at the CWC totals 2,627 cubic meters. An est imated 8,300 cubic meters of 1iquid have been "removed" through evaporation and solidification. This comprises all of the wastes that were in the basins.

\subsubsection{Characterization}

This section discusses the available waste characterization information. Information based on process knowledge and sample analyses is provided along with the waste designations and their bases, the uncertainty of the designations, and the schedule for further analysis.

3.9.2.1 Process Knowledge. The 183-H Basins received both routine and nonroutine waste. The routine waste stream consisted of spent acid etch solutions (primarily nitric, sulfuric, hydrofluoric, and chromic acids) generated by the nuclear fuel fabrication process. Typically, these acidic solutions were neutralized with excess sodium hydroxide before being transported to the 183-H Basins. Metal constituents in the waste included copper, silicon, zircontum, aluminum, chromium, manganese, nickel, and uranium. Following reaction with sodium hydroxide, these metals were present primarily in the form of precipitates. The resultant slurry of 1 iquid and metal precipitates was transported and discharged into the 183-H Basins.

Nonroutine waste also was discharged to the 183-H Basins during its period of operation. Before each addition, a review was performed to determine whether undesirable chemical reactions would take place. A "chemical waste disposal permit" system was developed for acceptance of waste into the 183- $H$ Basins. The permit system was for internal use only and should not be considered in the same context as a state or EPA permitted system. These internal chemical waste disposal permits have left a historical record that has been used to determine waste designations for the waste of the 183-H Basins.

Nonroutine waste consisted of unused chemicals and spent solutions from miscellaneous processes, development tests, and laboratories. Nonrout ine waste falls into three categories: listed waste, nonlisted waste that was added directly to the 183-H Basins, and nonlisted waste that was mixed with the routine waste stream before being transported to the 183-H Basins.

The chemical waste disposal permits have shown that six different listed nonroutine wastes were discharged into the 183-H Basins. Twelve chemical waste disposal permits were for the discharge of nonlisted, nonroutine waste directly into the 183-H Basins. This waste included sodium arsenate acid; ammonium phosphate; nickel oxide; mixed nickel, copper, and iron oxides; solutions of sodium nitrate, sodium sulfate (anhydrous), sodium chloride, and sodium carbonate (corrosive); sodium carbonate sludge; used boiler cleaning solution containing ethylene-diaminetetraacetic acid, ammonium persulfate, aqua ammonia, ethylene-diamine, hydrazine, and thiourea. 
A common practice for disposal of nonroutine wasite was to $\mathrm{mix}$ the materials with the routine waste strean before the waste was transported to the 183-H Basins. The chemical waste disposal permits indicate that about 44 cubic meters of 11quid waste and 1,545 kilograms of solid waste were discharged to the 183-H Basins in this manner.

Additional information is contained in the 183-H Solar Evaporation Basins Closure Plan (RL 1991c).

3.9.2.2 Sample Analyses. During the operating 1ife of the 183-H Basins, systematic chemical analyses were not performed for the routine waste discharges. In October 1984, the waste in Basin 1 was sampled. The waste contained three strata: a wet siudge, a 1iquid phase, and a relatively dry white stratum. In January 1986, the waste in Basin 2 was sampled. The waste consisted of a wet sludge and a liquid phase. During March 1987, the wet sludge and relatively dry crystali ine strata in Basins 3 and 4 were sampled. At the same time, the consolidated 1lquid (from Basins 1, 2, 3, and 4) in Basin 2 was also sampled (RL 1991c).

The waste in the inner portion of Basin 1 consisted primarily of sludge intermixed with a residual 1iquid. The cleanout effort involved pumping as much liquid as possible into Basin 2; therefore, the results for the Basin 1 1lquid are not discussed. The Basin 1 characterization was addressed by the analysis of the 1iquid in Basin 2. The outer basin waste was a relatively dry waste that was visibly different than the inner basin waste; consequently, samples taken from this stratum were analyzed separately. During removal of waste from Basin 1, no attempt was made to segregate the different stratum. Consequentiy, the most conservative designation resulting from the separate analyses was assigned to all waste from Basin 1.

The results of inorganic chemical analyses for major constituents showed that the waste consisted largely of sodium sulfate, along with water held as moisture and as water of hydration. Nitrate and fiuoride ions also were present in high concentrations. Copper constituted about $12 \%$ of the waste. The uranium concentration ranged from 390 to 530 parts per million (ppm).

Before removing sludge from Basin 2, samples of the liquid and sludge phases were analyzed for chemical constituents. The major constituents in the sludge were copper (13\%), sodium ion (9.7\%), and nitrate ion (13.5\%). Moisture content in the sludge averaged 53\%. Uranium was present in the sludge in concentrations up to $2,500 \mathrm{ppm}$.

The solid waste in Basins 3 and 4 was sampled concurrently, and the analytical results are similar enough to be treated in a single discussion. There were two visibly distinct waste strata in each basin. These consisted of a moist sludge (inner basin) and a relatively dry, white, crystalline stratum (outer basin) near the walls. Samples of the two strata were analyzed separately and each basin was sampled separately.

The sludge stratum in both basins consisted primarily of sodium, nitrate, and copper ions. Moisture content in this stratum averaged greater than $40 \%$ in each basin. The crystalline stratum contained high average concentrations of sodium and sulfate ions. A major difference between the basins was that 
the nitrate ion concentration in the crystalline stratum in Basin 4 ranged from 7 to $70 \%$, while in Basin 3 the levels were all less than $1 \%$. The urantum concentration ranged from 7 to 1,560 picocuries per gram dry weight.

Volatile organic analysis in accordance with Ecology and RCRA SW-846 methods was performed on 10 samples of wet sludge from Basins 3 and 4 . The primary reason for doing this analysis was to determine if tetrachloroethane and 1,1,1-trichloroethane (solvents routinely used in the nuclear fuels fabrication process) had reached the 183-H Basins via carryover into the rout ine waste stream. The analysis showed that neither solvent was present in detectable concentrations of 50 milligram per kilogram.

Five samples of the consolidated 1 iquid in Basin 2 were taken. The major constituents found were sodium and nitrate ions (14 and $38 \%$, respectively). Moisture content averaged 57\%. Uranium content for the liquid averaged 82,400 picocuries per iiter.

3.9.2.3 Waste Designation and Basis. The following are the bases for the waste designations:

- Pure chemical products identified on the internal chemical waste disposal permits

- Results of analyses conducted for characterizations of the waste for each basin.

The uranium content of the sludges and liquid is sufficient to classify them as non-TRU radioactive LLW.

Six listed wastes were discharged into the 183-H Basins. Five of these materials were extremely hazardous waste. All the 1 isted wastes were initially added to Basin 1. However, because of subsequent transfers of the liquids among the 183-H Basins, all 183-H Basins have been designated as having contained these 1isted materials. Consequently, waste codes applicable to all basin waste are U123 (formic acid), P030 (soluble cyanide salts), P120 (vanadium pentoxide), P029 (copper cyanides), P106 (sodium cyanide), and P098 (potassium cyanide). as follows:

Additional waste designations for waste of each of the 183-H Basins are

- Basin 1 (solid):

- Basin 2 (sludge):

- Basin 3 and 4:

- Basin 2 (1iquid):
WTO1 (fluoride ion concentration)

WT01 (fluoride ion concentration);

D007 (TCLP chromium)

WTO1 (fluoride ion concentration)

WTO1 (fluoride concentration); D007 (TCLP chromium).

3.9.2.4 Uncertainty of Maste Designation. The designations of the 183-H Basin waste are considered accurate. 
3.9.2.5 Schedule for Further Characterization. No further analyses are planned.

\subsubsection{Storage}

All solid and liquid wastes in the 183-H Basins have been removed and are being stored in the CWC. If other wastes are generated during closure, they will be handled in an agreed upon manner following regulatory requirements. Small concentrations of arsentc and lead have been found in the soll. A TCLP analysis on the berm soil has been completed and results show below regulatory levels for lead and arsenic. Therefore, this soll is not regulated as a dangerous waste.

It is DOE's intent to operate the CWC in compl tance with all applicable federal and state requirements related to mixed waste storage. Further details on this facility are provided in Chapter 3.0, Section 3.13. The storage unit compliance status of the CWC is discussed in Section 3.13.3.3.

\subsubsection{Treatment}

Treatment involved solidifying the liquids, packaging the solidified liquids and solid 183-H Basin waste for temporary storage, and moving it to the CWC.

All dangerous waste from the 183-H Basins will be retrieved for processing in the CWC's WRAP Facility, a multipurpose waste processing facility that is scheduled to start operation in 1996. The WRAP Facility as we11 as plans for treatment are described in Section 3.13.4.2.

\subsubsection{Waste Reduction}

The quantity of 183-H Basin waste requiring disposal has been reduced by solar evaporation. To minimize the waste generated when solidifying the remaining saturated, unevaporated 1iquid, 13 different liquid waste solidification agents were studied for packaging efficiency. The solidifying agent chosen provided a high-packaging efficiency, allowing 0.15 cubic meters of 1 iquid to be solidified and packaged into 0.21 cubic meters rather than 0.45 cubic meters as was the case with the older solidification agent.

\subsubsection{Variances, Exemptions, Time Extensions}

The 183-H Basins will undergo closure in accordance with an approved closure plan (RL 1991c) and the Hanford Facility RCRA Permit when it is finalized. The factlity will be clean closed, closed to health based standards in a modified closure, or closed as a landfill. The chotce of closure method currently is being evaluated. The dangerous waste and waste residues have been placed in containers and transported to the CWC for storage. This waste is managed with other waste stored at the CWC. 
The 183-H Basins waste consists of LLW containing dangerous waste constituents. The 183-H Bas in waste is restricted from 1 and disposal because it contains miscellaneous listed chemical waste (40 CFR 268.30) and California List waste (40 CFR 268.32). It al so contains waste covered by the Third-Third Promulgation (55 FR 22520).

The national capacity variance for Third-Third Waste (55 FR 22520) provided for a 2-year national capacity variance from the LDR for Third-Third mixed waste expiring on May 8, 1992. This variance allowed cont inued storage of these wastes. A Federal Register notice dated May 26, 1992, stated that EPA could not grant a case-by-case extension for Third-Third mixed waste because of questions regarding the DOE's demonstration that they have entered into binding contractual commitments to construct or otherwise provide treatment capability. A final dectsion has yet to be made. This extension would allow DOE to store LDR mixed wastes until May 8, 1993, and this date could be extended for up to an additional year. The Tri-Party Agreement will allow continued storage of these wastes in accordance with the schedules in the Tri-Party Agreement. The Federal Facility Compliance Act of 1992 also allows DOE facilities to store their wastes for 3 years if compliance with the Act's provisions for providing waste inventory and treatment plans are met.

The 183-H Basins' closure waste will be stored at the CWC until treatment by the WRAP Facility and subsequent disposal at appropriate disposal unit.

An additional variance also may be required to allow alternative treatment of waste code $\mathrm{U123}$ (formic acid), for which the required treatment is incineration or fuel substitution. Currentiy, there is no incineration capacity planned for mixed waste at the Hanford Site.

If additional variances; exemptions, or extensions of time are required because of delays in the development of treatment, storage, or disposal capacity, they will be applied for in accordance with the procedures detailed in the Tri-Party Agreement or regulations.

\subsection{PUREX STORAGE TUNNEL 2 MASTE (Mercury)}

This liquid LLW is contained in discarded dissolvers for irradiated fuel. The elemental mercury is sealed inside thermowells that are an integral part of the irradiated fuel dissolvers.

As of December $31,1993,0.01$ cubic meter of elemental mercury is stored in PUREX Storage Tunnel 2. The mercury is designated D009 (TCLP-mercury) and WTO1 (toxic) (RL 1990b).

\subsubsection{Generation}

Elemental mercury waste is generated when dissolvers in the PUREX process fail or are deemed to be obsolete (hereafter referred to as being discarded). The mercury becomes a waste because its removal from the discarded dissolver is not practical. 
The elemental mercury is sealed inside thermowells, which are an integral part of reactor fuel dissolvers used at the PUREX Plant. There are two thermowells per dissolver. Each thermowell consists of a 2.9-meter length of stainless steel pipe with an extension welded to the downside end. The lower end butts against the outer surface of the internal slotted bar screen that separates the undissolved fuel elements from the outer solution chamber of the annular dissolver. The mercury serves to transfer heat from the dissolver interior to the temperature sensor mounted within the thermowell. This mercury remains in the thermowells of discarded dissolvers. In preparation for storage, the thermowell is sealed with a stainless steel nozzle plug. In storage, the discarded dissolver rests in an inclined position in a cradle on a railca-. Secondary containment is provided by the dissolver vessel itself.

As of December 1993, three dissolvers have been discarded: one in 1971, a second in 1972, and a third in 1986. The first two dissolvers each contain 45 kllograms of elemental mercury, while the third one contains 38 kilograms. All three dissolvers are stored on railcars in PUREX Storage Tunnel 2 (RL 1990b).

\subsubsection{Characterization}

This section discusses the avaliable waste characterization information. Information based on process knowledge and sample analyses is provided along with the waste designations and their bases, the uncertainty of the designations, and the schedule for further analysis.

3.10.2.1 Process Knowledge. Characterization of the mercury waste relies on fabrication and installation specifications. The quantity of mercury present in each dissolver is documented on the fabrication drawings. None of the mercury will evaporate because each thermowell is sealed.

3.10.2.2 Sample Analyses. Sampling and chemical analysis is not performed on mercury associated with the dissolvers. The need for sample analyses will be evaluated furing planning for closure of the PUREX Plant including the PUREX Storage Tunnels. A Phase I closure plan will be submitted to Ecology about November 1994.

3.10.2.3 Maste Designation and Basis. The basis for the designation of mercury waste is process knowledge, and the fabrication and installation specifications.

Elemental mercury exhibits the characteristic of toxicity as determined by the TCLP and is designated D009. The quantity of mercury present, if exposed to a leachate, could produce an extract greater than 20 milligrams per 1iter. This dictates that the mixed waste be managed as extremely hazardous waste and is further designated as toxic (WTO1) (RL 1990b). The waste is also designated as ignitable (DOOI) and D011 (silver nitrate).

3.10.2.4 Uncertainty of Waste Designation. The designation of the PUREX Storage Tunnels mercury waste is considered accurate. 
3.10.2.5 Schedule for Further Characterization. The need for additional waste characterization will be evaluated during planning for closing the PUREX Storage Tunnels.

\subsubsection{Storage}

This section discusses the PUREX Storage Tunnels, provides their storage capacity and the amount of waste stored, and assesses the compliance status of the storage unit.

3.10.3.1 Storage Unit and Capacity. The PUREX Storage Tunnels are a mixed waste storage unit. The two tunnels are connected by rall to the PUREX P1ant and combine to provide storage space for 48 railroad cars (railcars). The PUREX Storage Tunnels provide long-term storage for process equipment removed from the PUREX PIant. Equipment transfers into the PUREX Storage Tunnels are made on an as-needed basis. Radioactively contaminated equipment is loaded on railcars and remotely transferred by rail into the PUREX Storage Tunnels. Rallcars act as both a transport means and a storage platform for equipment placed into the tunnels.

The tunnels are weather-tight structures covered by 2.4 meters of earth. This design serves to protect the stored equipment from exposure to natural elements, provides external radiation shielding from the radioactive equipment stored inside the tunnels, and provides for the protection of the environment.

Tunnel 1 (218-E-14) was completed in 1956 as part of the PUREX Plant construction project and provides storage for eight railcars. Tunnel 1 was filled to capacity (approximate?y 600 cubic meters of waste) in 1965 and subsequently was secured. There is no elemental mercury waste stored in Tunnel 1.

Tunnel 2 (218-E-15) was an expansion project constructed in 1964. This tunnel is different in design and is considerably longer than Tunnel 1 , providing storage space for a total of 40 railcars. A more complete description of the PUREX Storage Tunnels may be found in PUREX Storage Tunnels Dangerous Waste Permit Application, Rev. 1 (RL 1990b).

PUREX Storage Tunnel 2 has a maximum storage capactty of 40 railcars. Each rallcar will hold 77 cubic meters of waste. To date, $43 \%$ of the storage area is filled, as 17 railcars holding 1,300 cubic meters of discarded equipment and associated waste have been placed in the tunnel. Sufficient storage capacity exists for all future waste projected to be generated.

3.10.3.2 Amount In Storage. The amount of elemental mercury currently being stored in PUREX Storage Tunnel 2 is 0.01 cubic meter (128 kilograms).

3.10.3.3 Storage Compliance Assessment. Elemental mercury waste is stored in PUREX Storage Tunnel 2, a mixed waste storage unit. The PUREX Storage Tunnels do not have secondary containment structures; however, the mercury waste stored is contained in the thermowells of the dissolver vessels, and the outer shell of the dissolver provides secondary containment. Personnel entry (to inspect the waste storage area) is not practical because of the high levels of radiation present inside the tunnel, which would not meet the requirements of 
the Atomic Energy Act of 1954 to maintain radiation exposure as low as reasonably achievable. The PUREX Storage Tunnels Dangerous Waste Permit Application was submitted to Ecology in September 1990 in accordance with the Tri-Party Agreement (Ecology et al. 1992). No additional compliance actions have been identified for the PUREX Tunneis.

\subsubsection{Treatment}

3.10.4.1 Planned and Alternative Treatments. Planned treatment of the elemental mercury waste stored in PUREX Tunnel 2 is detalled in the Part B Dangerous Waste permit application (RL 1990b). The EPA required treatment technology for elemental mercury is amalgamation (52 FR 22520). Therefore, the treatment of choice is the current approach of adding zinc powder to create an amalgam. An alternative treatment being considered is to mineralize the elemental mercury (creating elemental mercury sulfide). After treatment, waste still classified as mixed waste will be placed in approved transport packaging and stored in an authorized Hanford Site storage unit or sent to a permitted mixed waste disposal unit.

3.10.4.2 Accelerated Treatment. A schedule for treatment of this waste has not been established. The material stored in the tunnels will be addressed as a part of PUREX Plant closure.

\subsubsection{Waste Reduction}

The elemental mercury in PUREX Storage Tunnel 2 will be separated from other waste categories to reduce the hazard of waste requiring processing and disposal as mixed waste.

\subsubsection{Variances, Exemptions, Time Extensions}

Elemental mercury waste was placed in the PUREX storage tunnels before November 1987 (the effective date of the LDRs for mixed waste) and, therefore, is not subject to LDRs until the waste is removed from the tunnels. Removal of elemental mercury waste is planned as part of the closure of the PUREX Plant. At that time waste will be removed from the PUREX storage tunnels, treated to comply with LDR treatment standards, and disposed of at a permitted disposal unit.

If variances, exemptions, or time extensions are required because of delays in the development of treatment, storage, or disposal capacity, they will be applied for in accordance with the procedures detailed in the Tri-Party Agreement or regulations.

\subsection{PUREX STORAGE TUNNELS 1 AND 2 MASTE (LEAD) AND} STORAGE TUNNEL 2 WASTE (SILVER)

The PUREX Storage Tunnels 1 and 2 contain 0.26 cubic meters of elemental lead and PUREX Storage Tunnel 2 contains 0.17 cubic meters. of silver (mostly as silver nitrate). The estimated volume of equipment associated with the 
elemental lead is 120 cubic meters. The estimated volume of equipment associated with the silver nitrate is 15 cubic meters (RL 1990b, Appendix A). The lead is in jumper counterweights and equipment weights, and the silver is in discarded silver reactors.

The elemental lead waste is TCLP toxic for lead (D008) and also is designated toxic (WTO1). The silver nitrate waste is classified as TCLP toxic for silver (DO11) and ignitable (DOO1) because of the presence of nitrates.

\subsubsection{Ceneration}

Elemental lead waste is generated in the PUREX process as an integral part of equipment, such as process pipe jumpers, jumper al ignment tools, and shielding equipment. Historically, elemental lead was used as weight, counterweight, and radiation shielding in the fabrication of process equipment used in the PUREX Plant; generally, the lead was encased in steel (carbon or stainless) to facilitate its attachment to process equipment. Counterweights are used to facilitate remote installation of in-cell process and service piping (jumpers). A jumper alignment tool may have contained as much as $680 \mathrm{kilograms}$ of lead. This tool is used as a weight to pull down the free end of a jumper so the connecting parts align vertically and the connection can be made.

Silver in the form of silver salts deposited on unglazed ceramic packing is contained within the discarded silver reactors stored in Tunnel 2 . Three silver reactors were used to remove radioactive iodine from the offgas streams of the irradiated reactor fuel dissolvers in the PUREX process. The silver reactor vessel contains two beds of packing. The packing is coated initially with 114 kilograms of silver nitrate used for iodine retention. Nozzles on the top of the reactor are provided to allow flushing and/or regeneration of the packing with silver nitrate solution as the need arises.

Experience has shown that after extended use, the silver reactors lose efficiency. This loss in efficiency normally occurs when about one-half the silver nitrate on the packing has been converted to silver fodide. Other competing reactions such as reduction of silver nitrate to metaliic silver and formation of silver chloride also occur and affect silver reactor efficiency. Therefore, regeneration of the silver reactor with fresh silver nitrate is performed periodicaliy. Thus, the packing of the discarded silver reactor contains a mixture of silver nitrate, silver halides, and silver fines.

During PUREX shutdown, elemental lead and silver nitrate waste may be generated by plant maintenance activities.

\subsubsection{Characterization}

This section discusses the available waste characterization information. Information based on process knowledge and sample analyses is provided along with the waste designations and their bases, the uncertainty of the designations, and the schedule for further analysis. 
3.11.2.1 Process Knowledge. The quantity of lead generated is determined from a review of fabrication and design drawings for each piece of equipment placed in storage if the lead weight, counterweight, or shielding is specifically detailed. The silver salts quantity is estimated from the knowledge of the amount of silver nitrate placed on the bedding and the regeneration history of the silver reactors. For accountability purposes, the total silver content is considered to be silver nitrate, the salt that exhibits the characteristics of both ignitability and TCLP toxicity.

3.11.2.2 Sample Analyses. Sampling and chemical analysis is not performed on lead or silver salts associated with the radioactive discarded equipment placed in the PUREX Storage Tunnels. The quantity of lead in storage is determined from process knowledge. Provisions for taking samples of the bedding were not provided in the design of the silver reactor vessels. Therefore, sampling and chemical analysis are not performed for silver salts before placing a silver reactor in storage.

3.11.2.3 Waste Designation and Basis. Elemental lead exhibits the characteristic of toxicity as determined by the TCLP and is designated D008. The form of lead present could produce an extract greater that 500 milligrams per liter should it be exposed to a leachate; therefore, the mixed waste is managed as extremely hazardous waste and is further designated as WTO1. However, because the bulk of the lead is encased in steel on railcars that isolate the lead from other materials stored within the tunnel, the potential for exposure of lead to a leachate is considered to be negligible.

Silver salts exhibit the characteristics of toxicity as determined by the TCLP and are designated DO11 as well as DOOI because of their oxidizer characteristics. The form of silver present could produce an extract having greater than 500 milligrams of silver per liter should the salts be exposed to a leachate; therefore, the mixed waste is managed as extremely hazardous waste and is further designated as WTO1. Although nitrate is an oxidizer and is designated 0001 silver (ignitable), the dispersion of a nitrate salt on unglazed ceramic packing contained within a stainless steel vessel and isolated from other materials stored within the storage tunnel results in a probability for ignition to be considered negligible.

3.11.2.4 Uncertainty of Maste Designation. The designated waste codes for the lead and silver waste are considered accurate.

3.11.2.5 Schedule for Further Characterization. No schedule for further characterization has been established. The need for additional waste characterization will be evaluated during planning for closing the PUREX Storage Tunnels.

\subsubsection{Storage}

The PUREX Storage Tunnels, their storage capacity, and the compliance status of the storage unit are discussed in Section 3.10.3. 
As of December 31, 1993, 0.26 cubic meters of elemental lead is stored in PUREX Storage Tunnels 1 and 2 , and 0.17 cubic meter of silver nitrate is stored in Storage Tunnel 2. The estimated volume of equipment associated with the elemental lead is 120 cubic meters. The estimated volume of equipment associated with the silver nitrate is 15 cubic meters (RL 1990b).

The amounts of lead and silver placed in the storage tunnels are given in Table 3-14. The estimated quantity of lead listed in Table 3-14 accounts only for the lead in alignment tool and jumper counterweights. Counterweights on equipment dunnage and lead used for shielding cannot be quantified by existing historical records and are not included in the estimated quantity of lead in storage.

The quantity of silver salts listed in Table 3-14 is a function of time of reactor use, the regeneration history, and the impurities in the process chemicals that may have reacted with the silver nitrate. Sample analyses have not been conducted to verify the predicted quantities present.

\subsubsection{Treatment}

3.11.4.1 Planned Treatment. Planned treatment of the elemental lead and the silver salts associated with the process equipment stored in the storage tunnels is presented in RL (1990b). The elemental lead will be removed, where feasible, from the process equipment to reduce the volume to be treated. The elemental lead, as well as the silver salts located in the silver reactors, are planned to be treated by encapsulating the material in a cementitious grout that immobilizes the lead and silver.

3.11.4.2 Treatment Alternatives. Alternatives to the process above have not been studied. As necessary, this will be done as part of the plant closure process.

3.11.4.3 Accelerated Treatment. A schedule for treatment of this waste has not been established. Waste from the tunnels will be handled along with the similar materials currently in the PUREX Canyon when PUREX is decontaminated and decommissioned. PUREX decontamination and decommissioning, along with treatment of the Tunnel waste, is contingent on several factors: the Sitewide Land Use PIan, the Sitewide Decontamination and Decommissioning Priority Schedule, the Environmental Impact Statement and public involvement comments must be completed. A basis for the treatment plan for the wastes associated with PUREX storage will be developed after all of the above are implemented.

\subsubsection{Waste Reduction}

Since early 198\%, the use of lead in the design and fabrication of new replacement equipment for the PUREX Plant has been discontinued wherever feasible.

The silver and elemental lead in the PUREX Storage Tunnels will be separated from other waste categories to reduce the hazard of waste requiring processing and disposal as mixed waste. 


\subsubsection{Variances, Exemptions, Time Extensions}

Elemental lead waste, silver nitrates, silver salts, and silver fines (mixed LLW) were placed in the PUREX storage tunnels before November 1987 and are, therefore, not subject to LDRs until the waste is removed from the tunnels. Removal of elemental lead waste and silver nitrates, silver fines, and silver salts is planned as part of the PUREX Plant closure. At that time waste will be removed from the PUREX Storage Tunnels, treated to comply with LDR treatment standards, and disposed of at a RCRA-compliant disposal

facility.

If variances, exemptions, or time extensions are required because of delays in the development of treatment, storage, or disposal capacity, they will be applied for in accordance with the procedures detailed in the Tri-Party Agreement or regulations.

\subsection{PUREX CONTAINMENT BUILDING (LEAD AND CADHIUM)}

Discarded process equipment removed from service in the PUREX PI ant and known to have shielding, weights, and/or counterweights containing elemental cadmium or lead are stored on the canyon deck within the containment building of the 202-A PUREX Building. A change in storage designation from a "waste pile" to "containment building" was made on November 24, 1992. The addition of waste cadmium storage to the canyon deck was also made on this date.

Segregation of lead in this way began in December 1987. The current inventory (as of December 31,1993 ) is approximately 0.284 cubic meters (approximately 3,226 kilograms) of radioactively contaminated lead (mixed waste). The waste cadmium and lead stored in the containment building currently is untreated. Of this 0.284 cubic meters, approximately 0.25 cubic meters also contain 6 kilograms of metallic cadmium. The preferred disposal option is microencapsulation.

\subsubsection{Generation}

The PUREX Plant is located in the 200 East Area of the Hanford Site. It processed irradiated nuclear fuel by separating usable actinides from fission products. The PUREX PI ant was constructed in 1955 and had operated intermittently as needed since then.

The lead in the PUREX Containment Buflding consists of material that had been used for shielding, weights, or counterweights in the PUREX PIant. In most cases, the lead is totally enclosed in steel. However, some of the lead sheeting used in shielding is unclad. Since early 1987, the use of lead in the design and fabrication of new or replacement equipment for the PUREX Plant has been discontinued wherever feasible.

The cadmium was used as neutron shielding and is totally enclosed in steel along with approximately 1,300 kilograms of lead. 
Specific equipment items that use protective radiation shielding include certain diaphragm-operated valves and neutron monitors used for process control. The amount of lead required for such purposes varies from about 270 kilograms for the shielding around a small diaphragm-operated valve to as much as 1,300 kilograms of lead for a single neutron monitor.

Massive lead weights, up to 680 kilograms, are used as jumper alignment tools in the remote installation of some jumpers. Such tools assist in the vertical alignment so connection can be made. Jumpers are rigid lengths of pipe used to connect lines providing solution transfer to and from process equipment. Counterweights ait attached to some of the jumpers to provide proper balancing for remote installation by the overhead maintenance cranes. A typical jumper counterweight consists of appropriately sized steel pipe filled with lead shot (approximately 45 kilograms) and welded shut on both ends.

LDR-regulated lead waste may be generated at the PUREX PIant during shutdown, but data are not avallable to estimate this generation rate. Lead waste may be produced from canyon equipment as the canyon equipment is moved from its current location to the Canyon deck. The amount of lead waste, which will be generated during deactivation when quantified, will be added to the LDR report.

\subsubsection{Characterization}

This section discusses the waste characterization and the basis for the waste characterization. The waste designation, the uncertainty of the designation, and the schedule for further characterization also are provided.

3.12.2.1 Process Knowledge. The waste comes from discarded radioactive process equipment with lead shielding, weights, or counterweights. The waste is characterized as cadmium or lead based on knowledge of the amount and material used to manufacture a specific equipment component as determined from review of the fabrication and design drawings for each piece of discarded equipment.

3.12.2.2 Sample Analyses. No chemical analysis of the waste has been performed and is not required because the waste is accurately characterized based on process knowledge.

3.12.2.3 Waste Designation and Basis. The waste (elemental cadmium and iead) is designated TCLP toxic for lead (D008), cadmium (D006), and toxic (WTO1). The material is a solid, noncombustible metal.

3.12.2.4 Uncertainty of Waste Designation. The waste designation is accurately known, based on process knowledge.

3.12.2.5 Schedule for Further Characterization. No further characterization of this waste is scheduled. 
DOE/RL-94-21

\subsubsection{Storage} status.

This section describes the storage unit and assesses its compliance

3.12.3.1 Description of Storage Unit and Capacity. The PUREX containment building is a portion of the plant with a thick concrete floor, walls, and ceiling (up to 1.8 meters thick). Work in the canyon is generally performed remotely because of high radiation levels.

Discarded process equipment with cadmium: and/or lead attachments are stored on the south side of the canyon. Periodically, lead-containing components are cut from the equipment and placed in a metal box suitable for transfer by rallcar into the PUREX Storage Tunnels. The remaining nonlead-containing equipment components are disposed of as LLW.

Because the waste in the containment building is located inside the 202-A Building, the waste is protected from external environmental forces such as wind, rain, and run-on flooding. A system of drains and sumps ensures that any liquids from the waste are routed to appropriate waste storage tanks.

3.12.3.2 Amount in storage. The combined quantity of lead and cadmium waste in storage is 0.31 cubic meters $(3,226$ kilograms of lead and 5.90 kilograms of cadmium). No additional lead has been added to storage since October 1990.

3.12.3.3 Storage Compliance Assessment. Containment building storage of mixed waste on the canyon deck of the 202-A Building is addressed in revisions of the Part A Permit application for the PUREX Plant. The PUREX Plant waste management unit was reviewed for compliance with interim status dangerous waste regulations in accordance with Tri-Party Agreement (Ecology et a1. 1992) Milestone M-21-00. No interim status compliance deficiencies were noted.

Submittal of a Part B Permit application or closure plan for the PUREX Plant has been deferred until July 1995, per Tri-Party Agreement Milestone M-20-24.

\subsubsection{Treatment}

3.12.4.1 Planned and Alternative Treatments. Although treatment units could be built to separate the contained lead and/or cadmium from its encasement and possibly refine the metal to remove radioactive contamination, it is doubtful if unrestricted release of the refined lead could be achieved. Therefore, the preferred treatment alternative currently is identified as microencapsulation (55 FR 22520). Other alternatives have not been studied at this time.

3.12.4.2 Accelerated Treatment. A schedule for treatment of this waste has not been established. The material stored in the tunnels will be addressed as a part of the PUREX Plant closure. 


\subsubsection{Waste Reduction}

Since early 1987, the use of lead counterweights in the design and fabrication of new or replacement equipment for use in the PUREX Plant has been discontinued wherever feasible. Nondangerous materials such as carbon or stainless steel are substituted for lead counterweights wherever practical.

\subsubsection{Variances, Exemptions, Time Extensions}

The national capacity variance for Third-Third Waste (FE FR 22520) provided for a 2-year national capacity variance from the LDR for Third-Third mixed waste expiring on May 8, 1992. This variance allowed continued storage of these wastes. A Federal Register notice dated May 26, 1992, stated that EPA could not grant a case-by-case extension for Third-Third mixed waste because of questions regarding the DOE's demonstration that they have entered into binding contractual commitments to construct or otherwise provide treatment capability. A final decision has yet to be made. This extension would allow DOE to store LDR mixed wastes until May 8, 1993, and this date could be extended for up to an additional year. The Tri-Party Agreement will allow continued storage of these wastes in accordance with the schedules in the Tri-Party Agreement. The Federal Facllity Compliance Act of 1992 also allows DOE facilities to store their wastes for 3 years if compliance with the Act's provisions for providing waste inventory and treatment plans are met.

Removal of the mixed waste stored in the containment building will be addressed as part of the PUREX Plant closure. At that time, waste will be removed from the PUREX canyon deck, treated to comply with LDR treatment standards, and disposed of at a permitted disposal facility.

If variances, exemptions, or time extensions are required because of delays in the development of treatment, storage, or disposal capacity, they will be applied for in accordance with the procedures detailed in the Tri-Party Agreement or regulations.

\subsection{CENTRAL MASTE CONPLEX STORED LON-LEVEL, TRANSURANIC, AND POLYCHLORIMATED BIPHENYL WASTE}

The CWC receives radioactive solid waste and provides temporary storage until treatment at the Hanford Site.

Waste is received at the CWC from all radioactive waste generators at the Hanford Site and any offsite generators that are authorized by the DOE to ship waste to the Hanford Site for treatment and disposal. The waste received at the CWC is generated by ongoing Site operations (e.g., PFP operation, waste management) and research and development activities conducted at the site (e.g., SST waste sampling and analysis). Offsite waste has been primarily from DOE research facilities and other DOE sites. The characteristics of the waste received at the CWC vary greatly from waste that is nondangerous LLW to TRU dangerous waste. The CWC currently stores, as of December 31, 1993, 
approximately 2,616 cubic meters of mixed LLW subject to LDRs and 122 cubic meters of TRU mixed waste subject to LDRs. Other dangerous waste that is not restricted from land disposal is stored at the CWC and is not included in these figures.

No treatment units currently exist for TRU or LLW contaminated with PCBs. Therefore, this waste is being held in storage at the CWC until treatment capability exists. The Hanford Site PCBs inventory includes contaminated liquids (PCB-contaminated hydraul ic fluid), contaminated combustible solids, and contaminated equipment (transformers, capacitors, and fluorescent light ballasts). There currently are 185.6 cubic meters of PCB-contaminated LLW and 78.4 cubic meters of PCB-contaminated TRU waste (as of 12/31/93).

An internal assessment completed in July 1992 identified container mismanagement problems at several generating units. A backlog of waste had been accumulating at generating units. The assessment revealed that some of the waste was potentially dangerous and had accumulated in excess of the 90-day regulatory 1 imit. To correct these mismanagement problems, a Backlog Waste Program was initiated in October 1992 to ship the waste to compliant storage or disposal. More than 5,000 containers were managed through this program. These containers were broken down into three subsets.

The first subset of the backlog waste was labeled as "unknowns." These containers did not have enough characterization information to manage the containers at a treatment, storage, or disposal facility. Approximately 259 containers were included under this subset. These containers were shipped to $T$ Plant for opening, sampling, and repackaging. Approximately 201 unknown drums have been processed and were shipped to the CWC in 1993. Repackaging of 58 unknown boxes was completed in February 1994.

The second subset of the backlog waste was waste that was sent to the CWC under a two-stage program. Approximately 2,649 containers were handled under this subset. The first stage was labeled "interim staging." Under this stage, worst case characterization was used for all the waste to ship it to compliant storage. The purpose of the first step was to ship the waste to a central location where it could then be managed under the second phase of the program. The second phase of the program is labeled "confirmation" and is designed to confirm the process knowledge and accept the waste under WHC (1993f).

During the completion of the first phase, Ecology issued a fine and compliance order against DOE-RL and WHC for the management practices in the tank farms that caused the waste to be managed improperly and led to the initiation of the backlog waste program. As part of the compliance order, completion of the second phase of the backlog program was ordered to be completed by September 1,1994. The criteria of the second phase were negotiated with Ecology and are defined under Waste Analysis? I an for Confirmation or Completion of Tank Farms Backlog Waste Designation (RL 1993a).

The remainder of the "interim-staged" containers are being handled in a similar manner to the requirements of RL 1993a and should be completed this year. 
The third and final subset of the waste is containers that had complete characterization information and were shipped to storage or disposal under the requirements of WHC (1993f). No further actions are required for this waste.

\subsubsection{Generation}

This section describes the generation of RMW and radioactive PCB waste shipped to the CWC.

3.13.1.1 Mixed Waste Generation. The majority of waste shipped to the CWC is generated in small quantities by routine plant operation and maintenance activities. Specifying generation rates and types of waste generated by each plant is difficult because this waste is not generated as a direct result of process operations. The overall volumes of mixed waste projected to be generated are given in Table 3-15. No data are available on the fraction of this waste that w111 be subject to LDRs, but the majority of this newly generated mixed waste probably will be subject to the LDRs. The dangerous waste designation of each container of waste is determined at its point of generation based on process knowledge of the waste placed in the container or sample analysis if sufficient process knowledge is unavailable. The major plants that generate mixed waste that is LDR and the general type of waste they generate are discussed below.

In the past the PUREX Plant, located in the 200 East Area, was used to process irradiated nuclear fuel from $N$ Reactor. The PUREX process uses a nitric acid solution to dissolve the fuel and a solvent extraction process to separate the various fission products from the uranium, plutonium, and neptunium product streams. Radioactive solid waste is generated in all parts of the PUREX PIant from routine laboratory operations to equipment maintenance. Typically, the mixed solid waste generated at the PUREX Plant includes lead shielding, decontamination solvents, mercury-filled light tubes, and other nonroutinely generated radioactive solid waste.

The PFP, located in the 200 West Area, has been used to process plutonium nitrate solutions from the PUREX Plant, plutonium oxide, and plutonium scrap into metal. The plant consists of several facilities, including the plutonium Reclamation Facility, the Remote Mechanical ' $C$ ' Line (RMC), and the Product Handling Facility. Several radioactive mixed waste streams including lead, PCBs, and laboratory wastes are routinely generated at the PFP and shipped to the CWC.

The Uranium Oxide Plant, located in the 200 West Area, converts uranyl nitrate solution generated from the reprocessing of $N$ Reactor fuel to uranium oxide solids that are shipped offsite for reuse. The primary source of mixed waste at the Uranium Oxide Plant is solvents and mineral acids $\left(\mathrm{HNO}_{3}\right.$ and $\mathrm{H}_{2} \mathrm{SO}_{4}$ ) used for decontamination or equipment maintenance in radiation areas. Other sources of LDR mixed waste at the Uranium Oxide Plant include contaminated fluorescent tubes and falled equipment. 
The 222-S laboratories, located in the 200 West Area, are used to analyze radioactive samples in support of waste management operations and tank characterization. These operations generate both solid and liquid mixed LLW. The solid waste generated by this laboratory includes the following:

- Radioactively contaminated lead

- Outdated chemicals and reagents

- Equipment and absorbent materials contaminated with radioactive waste.

The liquid mixed LLW is generated when using organic solvents to analyze radioisotopes.

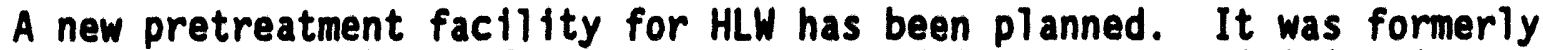
proposed to modify B Plant for this purpose. Maintenance activities in B Plant generate small quantities of solid waste, such as lead shielding, equipment decontamination agents, paint and painting supplies, and fluorescent light ballasts. This contact handled and remote handled waste is generated on an as-needed basis because of plant maintenance and upgrading.

T Plant, located in the 200 West Area, is used to decontaminate failed equipment to facilitate repair, reuse, or disposal of this equipment. The solid waste generated as a result of these operations includes spent solvents, failed equipment, lead shielding, paint and painting supplies, and metallic vapor lights.

N Reactor, located in the $100 \mathrm{~N}$ Area, is shut down in deactivated status. There are numerous sources of mixed LLW in the 100-N Area that generate waste oils, solvents, and decontamination solutions that in the past have been determined to be dangerous waste. In addition, the 100-H Area is the location of the 183-H Solar Evaporation Basins (Section 3.9), which was the source of a large quantity of waste (approximately 460 cubic meters).

The 300 Area Fuels Manufacturing Operations generates several mixed LLW streams. These operations have been shut down since December 1986, and the only waste generated from these operations is from decontaminating and closing these operations. A detailed description of the waste is provided in Section 3.16. The waste is being transferred to the CWC, or offsite if determined nonradioactive, as part of the closure activities for the 303-K Facility.

The FFTF, in the 400 Area, and associated research and development activities generate several waste streams that are mixed LLW. This waste includes waste sodium, which is discussed in Section 3.5, spent ethyl alcohol waste, listed solvent residual waste, contaminated lead residual waste, and decontamination waste. Spent ethyl alcohol waste is generated by cleaning of Materials Open Test Assembly specimens to remove residual sodium. This waste exhibits the characteristic of ignitability (D001) and corrosivity (D002). Listed solvent residual waste is generated by the use of 1 isted solvents in plant maintenance activities, such as manipulator repair and painting. Contaminated lead residual waste is generated from the removal of lead shielding for repair and replacement. Decontamination waste is generated 
while decontaminating stainless steel components, such as shipping casks, hot cells, or other equipment in the conduct of Fuels Material Examination Facility operations. The waste contains 1 isted solvents and may contain sufficient concentrations of chromium, nickel, and silver to be designated TCLP toxic.

The research and development activities conducted by PNL in the 300 and 3000 Areas generate numerous small-volume mixed waste streams that are 1 and disposal restricted. This waste is generated in the $303-C, 320,324,325$, 326, 327, 331, and 3720 Butldings. The laboratory waste may contain materials that are designated TCLP toxic (D003-D011) or that are designated as ignitable (D001) or corrosive (D002). The waste designated as TCLP toxic is generated from the analysis of samples containing toxic metals and the disposal of contaminated equipment and lead shielding. The waste designated as corrosive or ignitable is generated by using scintfllation cocktails containing ignitable solvents for the analysis of radionuclides.

The operation and maintenance of the SST and DST tank farms located in the 200 Areas generates several types of mixed waste. The waste includes equipment used for tank sampling and characterization, falled equipment and instrumentation, and small quantities of tank waste absorbed on clothing or rags. These waste streams may be designated by some or all of the waste codes applicable to DSTs. These codes include corrosivity (D002); TCLP toxicity for arsenic (D004), bartum (D005), cadmium (D006), chromium (D007), lead (D008), mercury (D009), selenium (DO1O), and silver (D011); spent halogenated solvents (F001); spent nonhalogenated solvents (F003); methyl ethyl ketone (F005); and toxicity (WTO1 and WTO2); Carcinogenic (WCO1 and WCO2), and persistent (WPO1 and WPO2).

3.13.1.2 Polychlorinated Biphenyl Waste Generation. The PCB-contaminated TRU and $L L W$ is generated by maintenance and perlodic flushing of PCB hydraulic systems, fallure of transformers and capacitors, and removal of PCB ballasts from light fixtures located in radioactive contaminated areas. The waste is packaged and shipped as solid waste to the CWC for storage.

The best avallable generation information is maintained in the computerized Solid Waste Information and Tracking System database. The Solid Waste Information and Tracking System contains only information provided by the waste generator. In the past, exhaustive waste descriptions that could be used to accurately classify a waste were not required, and data entries such as "contaminated debris" and "mixed fission products" were common. Data from the database indicates that 185.6 cubic meters of PCB-contaminated LLW and 78.4 cubic meters of PCB-contaminated TRU waste were generated bf ween 1970 and December 1993.

Future generation of PCB-contaminated waste is expected to be variable. The generation of this waste stream is correlated with the fallure rate of $P C B$ transformers, capacitors, and fluorescent light ballasts. Additional generation may be related to general Hanford Site cleanup and decontamination/ decommissioning activities. Sitewide cleanup efforts may identify soil-contaminated areas that will require cleanup and packaging. 


\subsubsection{Charactorization}

This section discusses waste characterization based on process knowledge and sample analysis, identifies known designations, and addresses any further characterization requited or planned.

Before acceptance of any waste at the CWC, it is characterized and packaged as described in Hanford Site Solid Waste Acceptance Criteria (WHC 1993f). These criteria require that the generator of the waste characterize each individual container of waste with sufficient accuracy to permit proper segregation: treatment, certification, shipment, and storage.

3.13.2.1 Process Knowledge. The waste characteristics are determined by the waste generator based on documented knowledge of the process generating the waste or sampling, as appropriate. The generators of all waste shipped to the CWC are periodicaily audited to ensure that waste is being managed in accordance with Hanford Site Solid Waste Acceptance Criteria.

Process knowledge has been used to characterize PCB-contaminated TRU waste and LLW currently in storage. Equipment containing PCBs, such as hydraulic systems, transformers, capacitors, and fluorescent 1 ight ballasts have been identified clearly. These systems are managed in accordance with 40 CFR 761 and waste are immediately handled and packaged as PCB TRU waste or LLW material.

3.13.2.2 Sample Analyses. The waste characteristics are determined by the waste generator based on documented knowledge of sample analyses of the generated waste. The generators of all waste shipped to the CWC are audited periodically to ensure that waste is being properly characterized.

Hydraulic systems and transformers have been sampled to determine PCB concentrations. Any waste resulting from the management of these systems is designated based on the concentration of PCBs in the source system. Light ballasts are designated based on data from the manufacturers.

Additional sampling is planned when this waste is processed through the WRAP Facility.

3.13.2.3 Waste Designation and Basis. Waste at the CWC is designated based on the information provided by the generator, performed by the waste analysis organization as part of a waste acceptance evaluation in accordance with Hanford Site Solid Waste Acceptance Criteria (WHC 1993f), and recorded in the Solid Waste Information Tracking System database. This database includes Washington State and RCRA waste codes resulting from designations based on process knowledge and sample analysis. Waste codes have been entered into the database since 1988 . When the waste codes were not found in database reports, waste designation tables were used to assign codes to containers placed in storage before 1988 .

3.13.2.4 Uncertainty of Waste Designation. The designation of the waste stored in the CWC is considered accurate.

3.13.2.5 Schedule for Further Characterization. No further characterization is required to accurately designate the present waste for storage. For some 
of the wasce, additional characterization needs to be performed to determine proper treatment and disposal options. This characterization will be performed during processing at the WRAP Facility. Further characterization may be necessary for newly generated waste and/or as a result of changed regulations.

\subsubsection{Storage}

This section describes the storage units associated with the CWC and details the amount and characterization of tha waste stored in these units.

3.13.3.1 Description of Storage Units and Capacity. The storage units described below are included in the CWC.

- Flammable Mixed-Waste Storage Modules--Eight modules are operational to store flammable LLW, TRU waste, mixed LLW, and TRU-mixed waste with flashpoints below $38^{\circ} \mathrm{C}$. The total capactty is 2460.21 -cubic meter drums. The modules are small preengineered buildings with 16.3 square meters of floor space each.

- Mixed-Waste Storage Bulldings--Thirteen mixed-waste-storage buildings are operational to store all categories of mixed waste (including TRU). The floor space of each building is 372 square meters. Each will have a 1,000-drum equivalent capacity.

- Large Mixed-Waste Storage Facility--The large mixed-waste storage facility will be operational in five phases, from third quarter FY 1991 for Phase I through FY 1998 for Phase V. The large mixed-waste storage unit will store all categories of mixed LLW with an 11,000-drum capacity each for the Phases I, III, and IV buildings; 18,000 drums for Phase II; and 27,000-drum equivalents (both drum and box waste) for Phase $v$.

- Waste Unloading and Staging Area--This pad is 9,000 square feet in area and can hold approximately 2,500 drums stacked two high. This pad is not intended for long-term storage.

- Mixed-Waste Storage Pad--The mixed-waste storage pad is located adjacent to the radioactive mixed waste storage buildings and is used as interim storage area.

A plan view of the future and existing CWC units is shown in Figure 3-11.

The planned capacity of the CWC to store LLW and TRU mixed waste is 14,450 cubic meters. This capacity is adequate to store the current projected volumes of mixed waste to be generated through the year 1999, assuming no treatment of the stored waste. Current plans call for treatment of the mixed waste to begin in 1999, which will reduce the amount of waste in storage and make storage room available for newly generated mixed waste. The capacity of the CWC to store mixed waste is continually evaluated and additional storage buildings will be constructed if necessary to meet forecast capacity shortfalls. 
3.13.3.2 Amount in storage. The amount of dangerous waste restricted from land disposal currently in storage at the CWC as of December 1993 is 5,118 cubic meters. This includes 2,627 cubic meters of waste from the 183-H Solar Evaporation Basins (see Section 3.9).

As of December $31,1993,185.6$ cubic meters of PCB TRU waste has been placed in the CWC for storage. Existing storage capacity is judged to be adequate for any future generation.

As of December 1993, 78.4 cubic meters of PCB LLW has been placed in the 2401. Wullding for storage. Existing storage capacity is judged to be adequate for any future generation.

3.13.3.3 Storage Compliance Assessment. The CWC was reviewed for compliance with interim-status dangerous waste regulations during 1988.

The compliance assessment noted a specific area of noncompliance, the contingency plan. Compliance action schedules are being developed as part of the Tri-Party Agreement (Ecology et al. 1992). Interim status compliance was achieved in June 1990.

\subsubsection{Treatwent}

This section describes the treatment of the mixed waste currently stored in the CWC.

3.13.4.1 Description of Current Treatment. The waste in the CWC currently is not undergoing any treatment, but is in storage pending the construction and operation of the WRAP Facility. The PCB, TRU, and mixed LLW is being stored until an approved processing facility is available.

3.13.4.2 Description of Proposed Treationt. The waste currently stored in the CWC, excepting PCB waste, will be treated at the WRAP Facility. The WRAP Facility will be constructed in modules, with Module 1 operational in 1997 and proposed Module 2A operational in 1999. Module 1 will provide examination, characterization certification, and shipping for boxes and drums of contact-handled LLW and TRU waste, but only drums would be opened and processed. It will also provide for decontamination of sniall items, primarily for decontamination of drums and overpacks. Most mixed LLW will be characterized and repackaged pending processing in Module $2 A$.

Module $2 A$ would contain size reduction and mixed waste treatment processes. All stored and newly generated mixed LLW and secondary solids from the Effluent Treatment Facility will be processed. Mixed LLW and effluent treatment unit secondary solids will be characterized, treated, solidified, and repackaged. All nonorganic radioactive, mixed LLW will be treated and certified for disposal in accordance with ail regulations, including the LORs.

Low-level mixed waste requiring thermal treatment is expected to be sent to a commercial operation for treatment to LDR requirements if possible. This waste will be returned to the Hanford Site for burial in the RCRA trench. 
Module 2B, with an undetermined startup date, is for characterizing, treating, and repackaging as required to permit permanent disposal of newly generated TRU and suspect-TRU waste in containers too large or heavy to be handled in Module 1 and all remote-handled TRU waste.

The WRAP Facilities will provide the capability to process retrieved suspect TRU waste, certify newly generated TRU waste and LLW for disposal, process large and heavy items, and process radioactive mixed waste for permanent disposal. These capabilities will be in accordance with LDRs and Hanford Site disposal criteria for LLW and in accordance with WIPP waste acceptance criteria and TRUPACT 2 (TRU package transporter) transportatinn criteria for TRU waste. An engineering study for the WRAP Facility, Module 2A (WHC 1990b), examined the mixed waste streams that would feed the WRAP Facility, examined potentially applicable treatment processes, and evaluated five alternative processing configurations. Following is a discussion of the treatment process that will be included in the WRAP Facility for mixed waste.

A basic schematic showing potential nonthermal treatment of radioactive mixed waste streams with corresponding treatment processes is shown in Figure 3-12 for WRAP Module 2A. Small-scale unit processes include immobilization/stabilization for particulate wastes, including sludges and ion exchange resins, mercury amalgamation, lead encapsulation, debris vibratory grouting, and miscellaneous processes, such as drum handling and treatment of iqquids.

When drums enter the WRAP Facility, Module 1, they will undergo nondestructive examination and analysis, container opening and sorting, sampling, and compaction. The TRU and LLW drums will be opened and material sorted in separate enclosures, but the opening and sorting process will be similar. After entering the enclosure, each drum will be deheaded and tipped onto a sorting table, and the inner plastic liner opened. All sorting will be performed automatically, although some manual sorting through gloveports with extension tools can be performed.

For drums that have been identified as containing potentially noncompliant items based on real-time radiography examination or visual inspection, those items will be removed, placed on a transfer drum, and transferred to a transfer drum and then to the restricted management waste gloveboxes. Examples of noncompliant items include free or containerized liquids, high-efficiency particulate air (HEPA) filters, and large quantities of particulates, aerosol cans, and suspect radioactive mixed waste. The sorting table will have a liquid collection tank beneath for liquids that flow freely from the opened waste. Collected liquids will be transferred to the restricted management waste gloveboxes.

In the restricted management waste gloveboxes, several operations will be carried out by operators through gloveports with the aid of extension tools. Any materials suspected of containing dangerous constituents will be sampled, and the samples will be transferred to the Sample Management area for transfer to Hanford Site laboratories or elsewhere for analysis. Treatment and disposal methods will be determined on a case-by-case basis for materials identified as mixed waste. The process enclosure in Module 1 primarily will 
be for characterization of any identified mixed waste and limited processing primarily to certify waste for disposal in WIPP. Some mixed waste may be packaged and sent to be processed in Module $2 \mathrm{~A}$.

The restricted management waste operators will enter descriptive information on waste materials into the computer database, bar code labels will be applied to all drums exiting the processing area, and the drums will be routed back to nondestructive assay and nondestructive examination. Restricted waste management will include operations for the following:

- Mixed waste sampling

- Immobilization of particulates

- Absorption of liquids

- HEPA filter immobilization

- Pyrophoric material

- Reactive metal

- Aerosol cans.

The WRAP Facility, Module 2A, will contain the mixed waste treatment processes, which will provide for all necessary nonthermal treatment of mixed LLW. Waste received will include dry particulates, sludges, ion exchange resins, some special wastes (mercury and lead), and all types of debris waste. All waste containers will be accompanied by paperwork attesting to the physical, chemical, and radiological contents.

A7ternatives studied for WRAP, Module 2A, but not part of Title 1 design, are compaction, size reduction, and lead decontamination.

3.13.4.3 Treatment Alternatives for Mixed Organic Mastes. In addition to WRAP, Module 2A, it is proposed to design, construct, and operate a Module 2B as described in Section 3.13.4.2. This separation of Module 2 into the $2 A$ and 2B components has not been formally approved through the Tri-Party Agreement change request process. A diagram showing the various WRAP modules is shown in Figure 3-13.

A significant quantity of Hanford Site RMW will require thermal treatment. Thermal treatment is prescribed in 40 CFR 268, for radioactive RCRA and Toxic Substances Control Act of 1976 (TSCA) solid wastes. Thermal treatment is required for destruction of alpha-contaminated PCBs currently in storage at the Hanford Site. In addition, the existing and projected mixed waste inventory at the Hanford Site includes a significant quantity of RMW that contains 1isted, F-Coded, hazardous organics having concentration-based treatment standards for which incineration is the best demonstrated available technology (BDAT). 
The thermal treatment alternatives presently being considered for Hanford Site RMW include the following:

- Privatization for offsite thermal treatment

- Hanford Site-specific Project W-242 thermal treatment facility

- Offsite treatment at another DOE site.

During FY 1994, privatized thermal treatment will be given full planning emphasis. However, treatment of all of the RMW requiring thermal treatment by a private company or by another DOE site may not be possible. Based on present assessments of these alternatives, both may prove to have technical and regulatory limitations. Existing thermal treatment technologies are not designed to burn alpha-contaminated wastes. Transport of the RMW to offsite facilities may be subject to prohibitive regulatory requirements. In addition, existing offsite DOE thermal treatment facilities currently have restrictive waste acceptance criteria making thermal treatment of Hanford Site RMW in significant quantities impossible. At the end of FY 1994, a decision will be made regarding the feasibility of privatized thermal treatment. If privatized thermal treatment proves to be feasible, it will continue to be given full planning emphasis. Assessments of the alternative to treat waste at other DOE sites have indicated that the next best alternative to privatized thermal treatment is the installation of the Project $\mathrm{W}-242$ Thermal Treatment Facility.

Accomplishments related to an onsite themal treatment facility include the completion of an engineering study that included a review of available thermal technologies and recommended thermal technologies specifically applicable to the treatment of Hanford Site RMW. Using the results of the technology study as basis, a Hanford Site-specific thermal treatment facility study was also conducted. Although the site-specific study has not been completed, conceptual data regarding the scope, configuration, and cost of an onsite thermal treatment facility was generated. The technology study and the site-specific facility study were also the basis for a completed Hazard Classification study that designated the Project W-242 Thermal Treatment Facility as a moderate hazard facility.

Accomplishments related to the treatment of Hanford Site RMW at other DOE sites include completing an assessment of possibly shipping selected streams of Hanford Site RMW to Idaho National Engineering Laboratory (INEL) for thermal treatment in INEL's Waste Experimental Reduction Facility (WERF), which employs rotary kiln waste incineration. The assessment concluded that the WERF startup schedule and limited waste acceptance criteria were unacceptably restrictive to give this option further consideration. A cursory review of the feasibility of shipping Hanford Site RMW to DOE sites other than INEL, such as Oak Ridge National Laboratory (ORNL) and the Savannah River Site (SRS), indicated that restrictive waste acceptance criteria, as well as site-specific schedules for waste treatment, yielded similar conclusions.

The thermal treatment of Hanford Site RMW by a commercial entity is the currently favored alternative by Hanford Site management. Accomplishments related to thermal treatment privatization include an assessment of industrial capability and interest in treating Hanford Site RMW. This assessment 
concluded that there was extensive interested capability in the private sector to treat Hanford Site RMW, but no technology is presently available to treat the entire inventory of Hanford Site RMW. Privatization of thermal treatment is being given full planning emphasis during FY 1994. Concerns regarding the implementation of NEPA for the thermal treatment of significant quantities of DOE RMW of considerably variant quality at an offsite commercial facility suggest that the privatization alternative could prove unfeasible. If privatization proves unfeasible, the sum of previous assessment data suggests that the Project W-242 Thermal Treatment Facility should be given full planning emphasis.

3.13.4.4 Accelerated Treatment. It would not be practical to accelerate treatment of mixed waste beyond the scheduled dates for WRAP start up. Because of the large costs involved to design and build this facility, accelerated treatment would not be feasible.

\subsubsection{Waste Reduction}

A11 plants and processes that generate waste that is shipped to the CWC are required to have a waste minimization program and a LLW certification plan in place. The effectiveness and implementation of these programs are audited on a regular basis. Key elements of this program are described in Section 2.5.

\subsubsection{Variances, Exemptions, Time Extensions.}

The CWC contains waste that is restricted from disposal because it contains constituents on the California 11st (40 CFR 268.32), solvents (40 CFR 268.30), and waste identified by the Third-Third LDRs (55 FR 22520).

The national capacity variance for Third-Third Waste (55 FR 22520) provided for a 2-year national capacity variance from the LDR for third-third mixed waste expiring May 8, 1992. This variance allowed continued storage of these wastes. A Federal Register notice dated May 26, 1992, stated that EPA could not grant a case-by-case extension for Third-Third mixed waste because of questions regarding the DOE's demonstration that they have entered into binding contractual commitments to construct or otherwise provide treatment capability. A final decision has yet to be made. This extension would allow DOE to store LDR mixed wastes until May 8, 1993, and this date could be extended for up to an additional year. The Tri-Party Agreement will allow continued storage of these wastes in accordance with the schedules in the Tri-Party Agreement. The Federal Facility Compliance Act of 1992 al so allows DOE facilities to store their wastes for 3 years if compliance with the Act's provisions for providing waste inventory and treatment plans are met.

The Tri-Party Agreement requires treatment and disposal capacity wastes to be developed on the following schedule:

- Completion of WRAP Facility, Module 1; required to sort and repackage waste and initiation of operations by March 1997 (Milestone M-18-00) 
- Completion of WRAP Facility, Module 2A; required to provide waste treatment capabilities that minimize the land disposal of low-level radioactive and mixed waste by September 1999 (Milestone M-19-00 proposed).

If additional variances, exemptions, or time extensions are required because of delays in the development of treatment, storage, or disposal capacity or the demonstrated need for using alternative treatment technologies, they will be applied for in accordance with the procedures detailed in the Tri-Party Agreement or regulations.

The required treatment for PCB waste is incineration. Currently there are no facilities available for incineration of mixed PCB waste. Alternative treatments currently are being investigated. The PCB waste will be stored at the CWC until an equivalent treatment technology is demonstrated and approved by EPA and Ecology. If availability of required treatment will extend the length of PCB waste storage beyond the time allotted to treat and dispose of other CWC waste, a variance to the storage prohibition will be applied for.

\subsection{RETRIEVABLY STORED LOW-LEVEL AND TRANSURANIC MASTE}

Since 1970, defense materials production, research, and waste management have produced TRU waste. Before 1970 there were no regulations that defined or required separation of TRU waste and it was commingled and buried with LLW. Initially, the definition of TRU waste included any waste with suspect alpha contamination. This definition was later (1972) changed to include only waste containing greater than 10 nanocuries per gram of alpha-emitting isotopes with half-lives greater than 20 years. The definition was then (1982) changed to include only waste with greater than 100 nanocuries per gram of TRU radionuclides. TRU radionuclides are those having an atomic number greater than 92. Because existing technology in the 1970s could not determine the concentration of TRU radionuclides at 10 or even 100 nanocuries per gram, any solid waste that was suspected to be TRU was placed in retrievable storage (WHC 1989a).

Retrievably stored LLW is waste that was generated after 1980 and in 1987 or before, when use of retrievable storage units was terminated. The waste contained liquid organics that precluded disposal as solid LLW because of concerns about affecting the ion exchange capacity of the soil. This waste is stored in retrievable storage units in the same manner as retrievably stored TRU waste.

The retrievably stored waste at the Hanford Site was not segregated based on the physical or chemical characteristics of the waste. The waste containers are filled with mixtures of materials, such as failed process equipment including pumps, resin columns, and tanks; laboratory and room trash including paper, plastics, glassware, cloth, solidified liquids, and animal carcasses; and decontamination and decommissioning rubble including concrete, piping, and soils.

The waste is contained primarily in 0.21-cubic-meter drums and metal or wood boxes. Waste is also contained in casks, concrete boxes, concreted culverts, and other miscellaneous containers. 
Before 1986, TRU waste had been placed in a variety of storage configurations. These storage configurations consisted of shallow land trenches, concrete-lined " $V$ " trenches, and earth-covered asphalt pads and caissons (WHC 1991C). The TRU waste has been stored in the TRUSAF since 1986 (Section 3.15) and in the CWC since 1987 (Section 3.13).

The majority of the TRU waste stored in the 200 Areas is generated by onsite activities; however, some of the TRU waste is generated offsite and shipped to the Hanford Site for retrievable storage (RHO 1985). Approximately 15,000 cubic meters of TRU waste had been placed in storage in the 200 Areas in over 38,700 containers.

Also in the low-level category are naval submarine reactor compartments currently stored in the 200 East Area Burial Ground 218-E-12B, Trench 94. These defueled reactor compartments are intended for permanent disposal, without further treatment, in their current location. For this reason, the compartments are not included in the storage inventory tables, waste minimization sections, or treatment discussions of this report. Although the compartments currently are stored, permit applications have been filed to allow disposal. Two permits are required: one from Ecology for lead disposal in a dangerous waste disposal facility and one from the EPA for PCB disposal in a chemical waste landfill. As much of the PCBs and lead as practical have been removed. The remaining lead and PCBs are encapsulated within the sealed hulls of the compartments.

As of March 1994, 35 compartments were stored awaiting disposal. Additional reactor compartments will be shipped to the Hanford Site in the future.

\subsubsection{Generation}

Extensive process knowledge is not available for many of the containers that have been placed in retrievable storage. The best available information is maintained in the computerized Solid Waste Information and Tracking System database. The Solid Waste Information and Tracking System contains only that information provided by the waste generator. In the past, exhaustive waste descriptions that could be used to classify a waste accurately were not required and data entries such as "contaminated debris" and "mixed fission products" were common (WHC 1989a). Because of incomplete classification of waste in the past, it is estimated that $10 \%$ of the TRU waste may be mixed waste.

\subsubsection{Characterization}

This section discusses waste characterization based on process knowledge and sample analysis, identifies known designations, and addresses any further characterization required or planned.

3.14.2.1 Process Knowledge. Limited process knowledge has been used to characterize the TRU mixed waste currently in storage. In the past few years changing waste reporting, manifesting, and packaging requirements have greatly increased the availability of process waste data for what may be used to 
characterize waste. Information related to the physical, chemical, and radiological properties of newly generated TRU waste is available. This availability is anticipated to reduce the amount of sampling and treatment required to meet long-term storage packaging requirements.

3.14.2.2 Sample Analyses. Sampling for mixed waste constituents will be performed when the TRU waste is retrieved from storage for processing. All drums and boxes of TRU waste in interim storage will be opened. Each individual container will be sampled and these samples will be prepared for transport to analytical laboratories in the 200 West Area for analysis.

3.14.2.3 Waste Designation and Basis. A review of data on TRU waste in retrievable storage units identified many constituents in each waste container that are designated dangerous waste. Data entered since 1988 has the designation of the dangerous constituents of each waste package assigned. When the designation was not found in database reports, waste designation tables were used to assign a designation to the constituents identified in TRU waste placed in storage before 1988.

It is anticipated that additional TRU mixed waste will be identified when waste is retrieved from storage for repackaging for disposal (WHC 1989a).

3.14.2.4 Uncertainty of Waste Designation. There is high confidence in the accuracy of the designations for newly generated TRU waste material. 01der waste will require additional characterization before treatment and disposal.

3.14.2.5 Schedule for Further Characterization. In situ sampling of retrievably stored TRU waste was initiated in FY 1991 with characterization to be completed by FY 1994 (WHC 1989b). The purpose of the sampling is to assess the current and future integrity of the retrievabiy stored waste containers and analyze contents. These objectives will be achieved by visual and nondestructive examination of waste containers, retrieval, and nondestructive assay.

Additional sampling will be performed as necessary to adequately characterize suspected mixed waste when waste packages are retrieved and processed through the WRAP Facility.

\subsubsection{Storage}

This section describes the current storage units and inventories and assesses compliance with applicable regulations.

3.14.3.1 Storage Unit and Capacity. The waste stored in the retrievable storage unit is primarily contained in 0.21-cubic meter drums and boxes. Initially drums were painted; however, after 1982, galvanized drums were used to minimize corrosion attributed to high humidity in storage modules. Initially boxes were constructed of plywood and steel, later of plywood coated with fiberglass reinforced polyester, and currently of steel. Waste also is contained in casks, concrete boxes, concreted culverts, and other miscellaneous containers. These containers were placed in a variety of 
storage configurations. These storage configurations consisted of shallow 1 and trenches, concrete lined " $V$ " trenches, and earth-covered asphalt pads and caissons (WHC 19901) (Figures 3-14 and 3-15).

Retrievably stored TRU waste is located in the 218-W-3A, $-4 B,-4 C$, and 218-E-12B Burial Grounds. Newly generated (after 1985) TRU waste is stored in the TRUSAF and CWC storage buildings.

Four different container storage configurations were used for contact-handled TRU waste at the Hanford Site. The first storage configuration consists of waste drums stacked horizontally in a gravel-bcitom "V" trench. The waste drums were covered directly with soil. This storage configuration was used from 1970 through 1972.

The second storage configuration was an engineered concrete and metal storage structure known as the V-7 trench. In the V-7 trench, drums were stacked on a 45-degree angle. This storage concept proved too expensive to implement and was used only between June 1972 and March 1973.

The third configuration consists of wide bottom and " $V$ " trenches. In both cases it is unknown if the trench floor was covered with plywood and drums were stacked vertically or if it was placed similar to configuration one (Figure 3-15). Boxed waste in this configuration may contain shoring used to protect it from collapse because of soil pressure. This storage configuration was used in the 200 West Area 218-W 3A and 218-W 4B Burial Grounds starting in 1974.

The fourth configuration consists of wide-bottom trenches. This storage configuration is the same as the third except the floor is asphalt. This storage configuration was used in the 218-W-4B Burial Ground, trench 07 , from 1974 until 1980 and in the 218-W-4C Burial Ground from 1978 to the present.

Some of the waste mentioned above is remote-handled waste. In addition, small containers of remote-handled TRU waste are stored in buried caissons; these caissons no longer are used for newly generated waste. The caissons are reinforced concrete cylinders 2.7 meters in diameter by 3 meters high and are buried 4 meters below grade. The caissons have 0.9 -meter diameter inlet chutes, offset or convoluted to reduce radiation or "shine" from the contents (Figure 3-15). Caissons are equipped with electrically driven exhausters fitted with HEPA filters.

Because the practice of placing TRU waste in burial ground retrievable storage units was discontinued in 1986, and no additional waste is planned to be added, the storage capacity for this waste is adequate.

3.14.3.2 Amount in Storage. Approximately 15,440 cubic meters of waste have been placed in storage in the 200 Areas retrievable storage units. Of this volume, $14.1 \%$ or 2,184 cubic meters are known to be dangerous waste based on information contained in the Solid Waste Information and Tracking System. Additional waste may be redesignated as dangerous 1 and disposal restricted waste upon retrieval. 


$$
\text { DOE/RL-94-21 }
$$

3.14.3.3 Storage Compliance Assessment. The retrievable storage units were reviewed for compliance with interim status dangerous waste regulations during 1988. This section discusses past and present disposal practices and discusses the interim status compliance requirements.

Waste was placed routinely in the retrievable storage units in shallow unlined trenches since 1960. Radioactive liquid organic waste was placed in retrievable storage units from 1982 through 1987. Burial of mixed waste with dose rates less than 200 millirems per hour at the container surface was halted in 1987. Mixed LLW, after the waste has been processed to remove the hazardous constituent to LDR levels, will be placed in 1 ined trenches with leachate collection and removal systems. The TRU mixed waste eventually will be retrieved, treated to comply with LDR requirements at the WRAP Facility or other appropriate treatment unit, and disposed of at a permitted dangerous waste disposal unit.

The compliance assessment noted the following specific areas of noncompliance with interim status requirements:

- The contingency plan should be upgraded to account for unit requirements of dangerous waste management

- A plan to inspect mixed waste placed in retrievable storage units should be developed

- Dangerous waste containers and accessible mixed waste backlog should be labeled

- A burial box and cardboard compaction and segregation strategy should be developed

- Additional groundwater monitoring wells around the low-level burial grounds, which include the retrievable storage units, should be installed.

Compliance action schedules were developed as part of the Tri-Party Agreement (Ecology et al. 1992). Compliance with contingency plan upgrade, inspection, and labeling requirements was achieved by June 1990. Use of cardboard boxes for burial was terminated effective January 1990 . Processing facilities for compatible wastes are currently available. Additionally, two groundwater monitoring wells were installed in 1993 for a total of 81 wells in the low-level burial grounds. Detailed information on these wells, cuttings, purgewater, and characterization data can be found the Borehole Completion Packages for the year in which the wells were completed (WHC 1991f, WHC 1992d).

The Part B Permit application, which documents the current compliance status with the dangerous waste regulations, was submitted in December 1989. Therefore, the retrievable storage units comply with the storage unit regulations as modified by the Tri-Party Agreement. 
DOE/RL-94-21

\subsubsection{Treatment}

This section describes the current and proposed treatment of retrievably stored TRU waste.

3.14.4.1 Current Treatment. No waste in retrievable storage units is being treated.

3.14.4.2 Proposed Treatment. Waste from retrievable storage units will be retrieved and shipped to the WRAP Facility. Organics will be burned in a planned incinerator or sent offsite for treatment. The WRAP Facility will treat waste so that it is acceptable for permanent disposal. Treatment activities include segregation of LLW and TRU waste from hazardous waste, repackaging waste, conducting nondestructive examination and nondestructive assaying of packaging, and certifying packages for shipment and disposal.

The WRAP Facility is proposed to be constructed as three modules with Module 1 operations in 1996, Module 2A operations in 1999, and Module 2B operational startup to be determined. Detailed descriptions of these modules, as well as treatment plans, are provided in Section 3.13.4. Module 2B is currently being reviewed as part of the M-33-00 Milestone.

\subsubsection{Waste Reduction}

The retrievable storage units no longer accept waste; therefore, a waste minimization program is not applicable. However, waste minimization will be considered when evaluating cleanup and disposal alternatives.

\subsubsection{Variances, Exemptions, Time Extensions}

The waste stored in the retrievable storage units after 1982 is restricted from land disposal because it contains California List waste (40 CFR 268.32) and/or solvent waste (40 CFR 268.30) and/or waste identified in the Third-Third Promulgation (55 FR 22520).

The national capacity variance for Third-Third Waste (55 FR 22520) provided for a 2-year national capacity variance from the LDR for Third-Third mixed waste expiring May 8, 1992. This variance allowed continued storage of these wastes. A Federal Register notice dated May 26, 1992, stated that EPA could not grant a case-by-case extension for Third-Third mixed waste because of questions regarding the DOE's demonstration that they have entered into binding contractual commitments to construct or otherwise provide treatment capability. A final decision has yet to be made. This extension would allow DOE to store LDR mixed wastes until May 8, 1993, and this date could be extended for up to an additional year. The Tri-Party Agreement will allow continued storage of these wastes in accordance with the schedules in the Tri-Party Agreement. The Federal Facility Compliance Act of 1992 also allows DOE facilities to store their wastes for 3 years if compliance with the Act's provisions for providing waste inventory and treatment plans are met. 
The Tri-Party Agreement requires treatment and disposal capacity wastes to be developed on the following schedule:

- Completion of WRAP Factlity Module 1, required to sort and repackage waste, and initiation of operations by March 1997 (Milestone M-18-00)

- Completion of WRAP Factlity Module 2A, required to provide waste treatment capabilities that minimize the land disposal of low-level radioactive and mixed waste by September 1999 (Milestone M-19-00).

If additional variances, exemptions, or time extenstons are required because of delays in the development of treatment, storage, or disposal capacity or the demonstrated need for using alternative treatment technologies, they will be applied for in accordance with the procedures detailed in the Tri-Party Agreement or regulations.

\subsection{TRANSURANIC WASTE STORAGE AND ASSAY FACILITY STORED WASTE}

TRU solid waste packaged in compliance with the WIPP/Waste Acceptance Criteria is stored in the 200 West Area, in the 224-T Buliding, also known as the TRUSAF.

\subsubsection{Generation}

The following are descriptions of current sources of TRU mixed waste.

- The PUREX PIant reprocessed irradiated fuel from $N$ Reactor. Radioactive solid waste collected from the PUREX Plant consists of room waste, such as gloves, paper, and plastics. The TRU portion is separated from the LLW. Some of the waste, such as mercury-filled light tubes, rags, and aerosol cans, are definitely dangerous and separate collection receptacles are established for collection of this waste. To ensure that dangerous waste is not inappropriately discarded with the LLW or TRU waste, the waste is sorted before packaging and shipment.

- The PFP routinely generates mixed solid waste. Fluorescent light tubes containing mercury are used in processing gloveboxes and radiation areas throughout the PFP. The majority of PCB ballasts and fluorescent light tubes are surveyed for radiological contamination and released. These waste streams are handled as hazardous waste. A small portion of the ballasts and fluorescent light tubes are radiologically contaminated and must be treated as mixed waste. Lead-lined gloves on processing gloveboxes are routinely replaced to minimize the potential for glove fallure and subsequent spread of radioactive contamination. Laboratory waste containing $x y l e n e$ and toluene are generated during the analys is of samples for neptunium and plutonium. The waste is packaged and shipped as solid waste. 
- Operations of the analytical laboratories in the 200 West Area generate TRU mixed waste. Included in this solid waste is radioactively contaminated lead, outdated solid commercial chemicals, and lead shielded waste from laboratory hot-cell operations.

- The PNL generates small quantities of TRU mixed waste from research operations that are fully characterized by process knowledge.

The TRUSAF received some containers of waste from offsite sources (Battelle Columbus, nito; Argonne National Laboratory, Chicago; Rocky Flats Plant, Colorado; and Lawrence Berkeley Laboratories, Cal ifornta). Onsite generation projections are 266 cubic meters annually. These containers are sent to TRUSAF for storage before their planned shipment to the WIPP. The TRUSAF only accepts waste certified for disposal at the WIPP that is packaged in 0.21-cubic meter drums. There is a moritorium on TRU waste shipment. The Governor of Washington submitted a letter to the Secretary of Energy stating that no TRU waste shipment into Washington State w111 be accepted unt11 the WIPP is opened.

\subsubsection{Characterization}

This section discusses waste characterization based on process knowledge and sample analysis, identifies known designations, and addresses any further characterization required or planned.

To be accepted at TRUSAF, waste must be packaged and characterized as described in the Hanford Site Solld Waste Acceptance Criteria (WHC 1993f). These criteria require that the generator of the waste characterize each individual container of waste with sufficient accuracy to permit proper certification, shipment, and storage. Kinds and quantities of dangerous constituents in the waste and physical and chemical characteristics of the waste must be known and recorded on appropriate forms.

3.15.2.1 Process Knowledge. The waste characteristics are determined by the waste generator based on documented knowledge of the process generating the waste. The generators of all waste shipped to TRUSAF are periodically audited to ensure that waste is being properly characterized. Currently, only three facilities (PUREX, PFP, and Strontium Semi-Works) are able to certify waste.

3.15.2.2 Sample Analyses. Samples are collected at the point of generation for any sample analysis required to adequately characterize for waste designation. No samples are collected at TRUSAF. Any waste that requires sampling will not be certified and consequently will be shipped to the CWC for storage and subsequent processing.

3.15.2.3 Maste Designation and Basis. The dangerous waste designation of each waste container is determined at its point of generation based on knowledge of the waste placed in the container.

3.15.2.4 Uncertainty of Maste Designation. The designations of waste stored in TRUSAF are considered to be accurate. 
3.15.2.5 Schedule for Further Characterization. Certified waste in interim storage is awaiting shipment to the WIPP. No further characterization is required for this waste.

\subsubsection{Storage}

This section addresses current storage units, describes inventories, and assesses compliance with applicable regulations.

3.15.3.1 Storage Unit and Capacity. The TRUSAF bullding was originally constructed to purify plutonium nitrate by the lanthanum fluoride process; it was idle for several years after new technolagy made it obsolete. In the early 1970s, the bullding was modified to meet requirements for storage of plutonium-bearing scrap and 1lquids. The cells in the processing areas have been completely sealed and isolated from the operating gallery and service areas. These operating and service areas have been stripped of all unnecessary control equipment, panelboards, and partitions to provide approximately 1,068 square meters of storage space on three floors (Figure 3-16). The unit storage capacity is 420 cubic meters $(2,000$ drums).

Accumulation of certified TRU waste in 0.21-cubic meter drums that exceeds the capacity of TRUSAF will be stored in the CWC. Future plans for the CWC include a TRUSAF replacement to be called Mixed Waste Storage Phase $V$, which will be operational concurrently with the WRAP Facility, Module 1.

3.15.3.2 Amount in storage. As of December 31, 1993, there are 43 cubic meters of TRU mixed waste stored in TRUSAF.

3.15.3.3 Storage Compliance Assessment. The TRUSAF unit was reviewed for compliance with interim status dangerous waste regulations during 1988 . The need for an upgraded contingency plan was identified and the plan was completed.

A Part B Permit application has been submitted.

\subsubsection{Treatment}

This section describes the current and proposed treatment of stored TRU waste.

3.15.4.1 Current Treatment. At TRUSAF, packaged waste is $x$-rayed (to ensure what can be identified generally agrees with the documentation) and assayed to determine TRU activity. All TRU waste packages that meet the WIPP/Waste Acceptance Criteria requirements are placed in interim storage pending shipment to the WIPP. Noncertifiable TRU waste is sent to the CWC or stored in the TRUSAF. When the WRAP Facility, Module 1, begins operating, nondestructive evaluation and assay activities will be transferred from TRUSAF to the WRAP Facility.

3.15.4.2 Proposed Treatment. Certified TRU waste in TRUSAF interim storage will be shipped to the WIPP for permanent storage. 
3.15.4.3 Treatment Alternatives. The waste is not planned to be treated. The WIPP facility will be the only facllity in the nation capable of permanent disposal of these wastes.

3.15.4.4 Accelerated Treatment. Current plans are to ship the waste to WIPP for permanent disposal. No treatment plans have been proposed. Acceleration of shipment to WIPP is not possible because WIPP has not yet opened.

\subsubsection{Waste Reduction}

All plants and processes that generate waste that is shipped to TRUSAF are required to have a waste certification program in place. The effectiveness and implementation of this program is audited on a regular basis. Key elements of this program are described in Section 2.5 .

\subsubsection{Variances, Exemptions, Time Extensions}

The national capacity variance for Third-Third Waste (55 FR 22520) provided for a 2-year national capacity variance from the LDR for Third-Third mixed waste expiring May 8, 1992. This variance allowed continued storage of these wastes. A Federal Register notice dated May 26, 1992, stated that EPA could not grant a case-by-case extension for Third-Third mixed waste because of questions regarding the DOE's demonstration that they have entered into binding contractual commitments to construct or otherwise provide treatment capability. A final decision has yet to be made. This extension would allow DOE to store LDR mixed wastes unt 11 May 8, 1993; and this date could be extended for up to an additional year. The Tri-Party Agreement will allow continued storage of these wastes in accordance with the schedules in the Tri-Party Agreement. The Federal Facility Compliance Act of 1992 also allows DOE faclitities to store their wastes for 3 years if compliance with the Act's provisions for providing waste inventory and treatment plans are met.

If additional variances, exemptions, or time extensions are required because of delays in the development of treatment, storage, or disposal capacity, they will be applied for in accordance with the procedures detailed in the Tri-Party Agreement or regulations. Treatment of radioactive mixed waste is expected to be initiated in 1999.

\subsection{3-K STORED WASTE}

The 303-K Radioactive Mixed Waste Storage Facility (303-K Facility) is located in the northwest portion of the 300 Area of the Hanford Site. Since 1943, the 303-K Facllity has stored various radioactive and dangerous process materials generated by fuel fabrication in the 300 Area (RL 1990C). The 303-K Radioactive Mixed-Waste Storage Facility has been used for the interim storage of the following mixed waste streams generated within the 300 Area:

- Spent degreasing solvents

- Zircaloy-2 and beryllium/Zircaloy-2 chips and fines 
- Precipitates from neutralization of acid wastes

- Miscellaneous uranium-contaminated hazardous materials.

Routine waste has not been added to the 303-K Facility since mid-1987.

\subsubsection{Generation}

This section describes the waste generation process and estimates the generation rate for the 303-K Facility. The 303-K Facility has been ir. operation since 1943 and continues today as an interim storage facility for dangerous and mixed waste generated by cleanup activities in the fuel manufacturing processing in the 300 Area.

The 303-K Facility has the capacity to store 200 drums that may contain waste designated D001, D002, D004, D005, D006, D007, D008, D009, D011, D029, D035, D037, D039, D040, F001, F002, F003, F005, WCO2, WPO1, WPO2, WTO1, and WTO2.

The 303-K Facility has been used since January 1986 to store containers filled with low-level radioactive wiste and mixed waste generated at other N Reactor fuel manufacturing buildings in the 300 Area. Before 1987 the waste that was potentially contaminated with uranium included waste oils and cutting lubricants, concreted waste froin the 304 Facility, salt crystals from the waste-acid tanks in Building 334-A, degreaser solvents, acid absorbed on opal clay, solids from the 313 Building waste-acid treatment process, and waste cutting oils with solvents from uranium machining operations in the 333 Building.

Approximately fifty to one hundred 0.21-cubic-meter drums of waste were accumulated at the 303-K Facility annually before 1987. The maximum estimated inventory of containerized waste stored inside the 303-K Facility at any time was 200 drums or 42 cubic meters of waste.

Current wastes stored or planned to be stored in the 303-K Facility and the approximate volume or weight to be generated are shown below.

- Approximately 23 kilograms of spent degreasing solvents (D008, D039, F001, F002, WPO1, and WTO1). This waste al so contains cobalt-60 and uranium. No future generation of waste degreaser solvent is anticipated.

- Approximately 800 kilograms of Zircaloy-2 and beryllium/Zircaloy-2 chips and fines before concreting the waste. This material is designated ignitable (DO01) because of its pyrophoric properties before concreting and is nonhazardous after concreting. About 30 kilograms of this waste was generated in FY 1993 and about 40 kilograms are anticipated to be generated in FY 1994. 
- One 0.73-cubic-meter metal box containing a belt sander contaminated with pyrophoric Zircaloy-2 fines and entombed in dry sand is designated as ignitable (D001). No future generation of Zircaloy-2 ignitable contaminated equipment entombed in dry sand is anticipated.

- Eight 30-gallon drums containing about 780 kilograms of filter press and in-line filter sludge were stored in 303-K in 1992. This waste contains sodium diuranate and is designated 0006 because of cadmium, D007 because of chromium, and D008 because of lead. One 55-gallon drum of centrifuge sludge waste is anticipated to be generated in FY 1995.

- One 55-gallon drum containing about 120 kilograms of sandy sludge from the outside process sewer drain sump on the southwest side of the 313 Building. This uranium-contaminated waste is designated D005 because of barium, D006 because of cadmium, D007 because of chromium, and 0008 and WCO2 because of lead. Two 55-gallon drums of this sump sludge are anticipated to be generated in FY 1994.

- One 55-gallon drum containing about 68 kilograms of uraniumcontaminated lead metal designated as 0008 . No further generation of this lead waste is anticipated.

\subsubsection{Characterization}

The 303-K Facility contains radiologically contaminated spent chlorinated solvents, filter press and in-line filter sludge, lead metal, process sewer sump sludge, and pyrophoric chips and fines from cleanup activities of the $N$ Reactor fuels manufacturing area in the 300 Area. Waste descriptions are provided in Table 3-16.

\subsection{Process Knowledge. Pyrophoric beryllium/Zircaloy-2 alloy and} Zircaloy-2 chips and fines in water-filled drums are awaiting concretion in the 304 Building. After concreting, the waste will be nonhazardous and will be sent to the low-level burial ground.

Spent degreaser solvents (consisting of perchloroethylene, 1,1,1-trichloroethane, and rinse water from vapor degreasers) were absorbed on pads and placed in steel drums in 1988.

3.16.2.2 Sample Analysis. The eight drums of filter press and in-line filter sludge were analyzed for $\mathrm{pH}$, uranium by laser fluorimetry, and inorganic constituents by inductively coupled plasma and ion chromatography.

The one drum of absorbed degreaser solvent remaining at $303-K$ was analyzed for volatile and semivolatile organics, pH, flashpoint, and metals (by inductively coupled plasma), and mercury, selenium, arsenic, and lead. The radioactivity was measured with PNL's barrel assayer.

The one drum of sandy sludge from the process sewer sump was analyzed for $\mathrm{pH}$, metals by inductively coupled plasma, inorganic constituents by ion chromatography, and uranium by activity counting. 
The 25 drums of pyrophoric uranium and Zircaloy-2 chips and fines were analyzed for metals by inductively coupled plasma and uranium by laser fluorimetry.

3.16.2.3 Waste Designation and Basis. The designation for waste currently stored at the 303-K Facility is based on analytical results and process knowledge of the $N$ Reactor fuel fabrication operations in the 300 Area:

- D008, D039, F001, F002, F003, WP01, and WTO1 for spent degreasing solvents

- D001 for Zircaloy-2 and beryllium/Zircaloy-2 chips and fines

- D006, D007, D008, and WCO2 for filter press and in-line filter sludge and process sewer sump sludge

- D008 for contaminated lead metal.

3.16.2.4 Uncertainty of Waste Designation. The designations of stored container wastes at the 303-K Facility are considered to be accurate.

3.16.2.5 Schedule for Further Characterization. Currently it is planned to sample in CY 1994 the one drum of pyrophoric fines placed in the

303-K Facility in December 1993 and any other drums of this material generated in CY 1994.

\subsubsection{Storage}

This section discusses the 303-K Facility waste storage and capacity, identifies stored quantities, and assesses the compliance status of the unit.

3.16.3.1 Storage Unit Capacity. The 303-K Facility has a total storage capacity of 200 drums or 42 cubic meters of waste.

3.16.3.2 Amount in storage. The amount of containerized mixed waste in storage (as of December 1993) in the 303-K Facility is 2,786 kilograms. Thjs includes 260.11 -cubic-meter drums, 90.21 -cubic-meter drums, and $10.73 \mathrm{~cm}^{3}$ metal box.

3.16.3.3 Storage Compliance Assessment. The 303-K Facility is currently scheduled for clean closure with an interim use as a less-than-90-day accumulation unit. The unit is currently operating under interim status as a RCRA treatment, storage, and disposal facility.

\subsubsection{Treatment}

This section discusses the 303-K Facility current and proposed waste treatment processes.

3.16.4.1 Current Treatment. The solvent, lead metal, and sludge wastes currently stored at the 303-K Facility will be transferred to the CWC for 
long-term storage until a final treatment or disposal option for the waste is established. The pyrophoric chips and fines will be concreted in CY 1994 in the 304 Building and sent to the low-level burial ground.

3.16.4.2 Proposed Treatment. Current plans for the existing inventories of waste solvents at the 303-K Facility call for treatment offsite beginning in 1999.

Treatment plans in FY 1994 for the one metal box containing the belt sander is to remove the sand and separate the pyrophoric fines from the su: faces of the belt sander. The removed pyrophoric fines will be concreted in the 304 Buflding and sent to the low-level burial ground. The belt sander, sand and metal box will be sent to the low-level burial ground.

3.16.4.3 Accelerated Treatment. Any plans for accelerated treatment of 303-K Facility waste will be made as part of planning for WRAP Facility operations (Section 3.13.4). This would include onsite or offsite treatment.

3.16.4.4 Treatment Alternatives. Alternatives treatments for the relatively small quantities of $303-K$ wastes will be considered as part of design and operating plans for the WRAP Facility. Concretion has been determined to be the best disposal option for the pyrophoric chips and fines.

\subsubsection{Waste Reduction}

Because $N$ Reactor fuel has not been fabricated since December 1986, the 303-K Facility no longer receives routine waste products for long-term storage.

Fifty-six of the 57 solvent waste containers stored at the 303-K Facility have been transferred to the CWC for interim storage until shipped offsite for treatment.

\subsubsection{Variances, Exemptions, Time Extensions}

The dangerous waste and waste residues are being placed in containers and transported to the CWC for storage, as discussed in Section 3.16.3. This waste will be managed with other CWC stored waste.

The national capacity variance for Third-Third Waste (55 FR 22520) provided for a 2-year national capacity variance from the LDR for Third-Third mixed waste expiring May 8, 1992. This variance allowed cont inued storage of these wastes. A Federal Register notice dated May 26, 1992, stated that EPA could not grant a case-by-case extension for Third-Third mixed waste because of questions regarding the DOE's demonstration that they have entered into binding contractual commitments to construct or otherwise provide treatment capability. A final decision has yet to be made. This extension would allow DOE to store LDR mixed wastes until May 8, 1993, and this date could be extended for up to an additional year. The Tri-Party Agreement will allow continued storage of these wastes in accordance with the schedules in the 
Tri-Party Agreement. The Federal Facility Compliance Act of 1992 also allows DOE facilities to store their wastes for 3 years if compliance with the Act's provisions for providing waste inventory and treatment $\mathrm{plans}$ are met.

If additional variances, exemptions, or time extensions are required because of delays in the development of treatment, storage, or disposal capacity, they will be applied for in accordance with the procedures detailed in the Tri-Party Agreement or regulations. 
DOE/RL-94-21

This page intentionally left blank. 
Table 3-1. Plutonium Finishing Plant Radioactive Liquid Waste Stream Composition. (sheet 1 of 2)

\begin{tabular}{|c|c|c|c|c|c|c|c|c|}
\hline & UNITS & $\begin{array}{l}\text { PRF } \\
\text { CAW }\end{array}$ & $\begin{array}{l}\text { PRF } \\
\text { CXPP }\end{array}$ & $\begin{array}{l}\text { PRF } \\
\text { CUU } \\
\end{array}$ & $\begin{array}{l}\text { RMC } \\
\text { HSW } \\
\end{array}$ & $\begin{array}{l}\text { PRF } \\
\text { LSW } \\
\text { SSH }\end{array}$ & $\begin{array}{l}\text { RMC } \\
\text { LSWH } \\
\end{array}$ & $\begin{array}{l}\text { D\&AL } \\
\text { LSH }\end{array}$ \\
\hline $\begin{array}{l}\text { Normal flow } \\
\text { Peak Flow } \\
\text { SpG }\end{array}$ & $\begin{array}{l}L / h \\
L / h \\
g / c c\end{array}$ & $\begin{array}{r}158 \\
250 \\
1.18\end{array}$ & $\begin{array}{r}18 \\
30 \\
1.05\end{array}$ & $\begin{array}{r}20 \\
25 \\
1.05\end{array}$ & $\begin{array}{r}25 \\
100 \\
1.27\end{array}$ & $\begin{array}{r}25 \\
110 \\
1.06\end{array}$ & $\begin{array}{r}66 \\
90 \\
1.03\end{array}$ & $\begin{array}{r}20 \\
200 \\
1.01\end{array}$ \\
\hline
\end{tabular}

Mominal Composition

Species Range

\begin{tabular}{|c|c|c|c|c|c|c|c|c|c|}
\hline $\mathrm{Al}^{++++}$ & ppom & 20,000 & $10,000-18,000$ & 0 & 0 & 0 & 0 & 0 & 0 \\
\hline$B 0^{++}$ & ppon & 1 & & 0 & 0 & 0 & 1 & 0 & 0 \\
\hline $\mathrm{Ca}^{++}$ & ppom & 50 & & 1 & 0 & 0 & 6 & 2 & 0 \\
\hline $\mathrm{Cr}$ & ppon & 70 & $10-100$ & $<.01$ & $<.01$ & $<.1$ & $<.01$ & $<.01$ & $<.01$ \\
\hline $\mathrm{Fo}^{++}$ & ppon & so & $0-10,000$ & 50 & 50 & 50 & 60 & 54 & 50 \\
\hline$H^{+}$ & ppen & 2,000 & $2,000-3,000$ & 0 & 400 & 0 & 2,000 & 800 & 100 \\
\hline$k^{+}$ & ppm & 40 & $0-7,000$ & 0 & 0 & 200,000 & 200 & 90 & 0 \\
\hline $\mathrm{Hg}^{++}$ & ppom & 1 & & 0 & 0 & 0 & 1 & 0 & 0 \\
\hline$m+$ & ppom & 50 & $0-9,000$ & 0 & 0 & 0 & 100 & 300 & 0 \\
\hline $\mathrm{No}^{+}$ & ppom & 600 & $0-10,000$ & 20,000 & 0 & 20,000 & 100 & 100 & 100 \\
\hline $\mathrm{Ni}^{++}$ & ppom & 40 & $10-100$ & $<.01$ & $<.01$ & $<.1$ & $<.01$ & $<.01$ & $<.01$ \\
\hline $\mathrm{Pb}^{++}$ & ppm & 90 & & 0 & 0 & 0 & 0 & 0 & 0 \\
\hline$s r^{+t}$ & ppm & 2 & & 0 & 0 & 0 & 0 & 0 & 0 \\
\hline $\mathrm{OH}^{\circ}$ & ppom & 0 & & 80 & 0 & 30,000 & 0 & 0 & 0 \\
\hline $\cos { }^{\circ}$ & ppm & 0 & & 30000 & 0 & 1,000 & 0 & 0 & 0 \\
\hline $\mathrm{Cl}^{\circ}$ & ppom & 300 & $0 \cdot 20,000$ & 3 & 3 & 9,000 & 200 & 30 & 10 \\
\hline$F^{-}$ & ppom & 800 & $300-1,000$ & 1,000 & 1,267 & 44,280 & 0 & 0 & 0 \\
\hline $1^{\circ}$ & ppom & 0 & & 0 & 0 & 0 & 0 & 0 & 0 \\
\hline $\mathrm{MO}_{3}^{\circ}$ & ppm & 300,000 & $(2 .-3) E 5$. & 20,000 & 20,000 & 0 & 100,000 & 50,000 & 1,000 \\
\hline $\mathrm{NO}_{2}^{-}$ & ppm & 1,000 & & 60 & 0 & 0 & 400 & 400 & 20 \\
\hline $\mathrm{PO}_{4}$ & ppm & 20 & & 2 & 0 & 0 & 8 & 4 & 0 \\
\hline $\mathrm{SO}_{4}{ }^{\cdots}$ & ppom & 200 & & 0 & 0 & 0 & 0 & 0 & 0 \\
\hline CC14 & ppm & 600 & & 700 & 700 & 0 & 300 & 0 & 0 \\
\hline Amonia & ppom & 200 & & 0 & 0 & 0 & 0 & 0 & 0 \\
\hline TBP & Ppom & 4,000 & & 6,000 & 6,000 & 0 & 0 & 0 & 0 \\
\hline BUTAMOL & Ppm & 12 & $19-13$ & 0 & 0 & 0 & 500 & 0 & 0 \\
\hline DBP & ppom & 0 & & 800 & 0 & 0 & 0 & 0 & 0 \\
\hline MBP & ppm & 0 & & 0 & 0 & 0 & 0 & 0 & 0 \\
\hline TOC* & ppen & 3,000 & & 3,000 & 3,000 & 0 & 400 & 0 & 0 \\
\hline silica & pen & 90 & & 0 & 0 & 0 & 0 & 0 & 0 \\
\hline
\end{tabular}


Table 3-1. Plutonium Finishing Plant Radioactive Liquid Waste Stream Composition. (sheet 2 of 2)

Nominal Composition

\begin{tabular}{|c|c|c|c|c|c|c|c|c|c|}
\hline Species & UNITS & $\begin{array}{l}\text { PRF } \\
\text { CAW }\end{array}$ & Range & $\begin{array}{l}\text { PRF } \\
\text { CXP } \\
\end{array}$ & $\begin{array}{l}\text { PRF } \\
\text { CUU } \\
\end{array}$ & $\begin{array}{l}\text { RMC } \\
\text { HSH }\end{array}$ & $\begin{array}{l}\text { PRF } \\
\text { LSW } \\
\end{array}$ & $\begin{array}{l}\text { RMC } \\
\text { LSW } \\
\end{array}$ & $\begin{array}{l}\text { DRAL } \\
\text { LSW }\end{array}$ \\
\hline $\mathrm{Pu}$ & ppm & 9 & $2-200$ & 60 & 20 & $<1$ & 2 & 6 & 0 \\
\hline Am & ppm & 1 & $0-8$ & 0 & 3 & 0 & 1 & 1 & 0 \\
\hline U & ppm & 1 & & 0 & 20 & 0 & 0 & 3 & 0 \\
\hline
\end{tabular}

CAH a CA colum equecus waste strecm.

CW = CU colum squeous waste strean.

CXP = CX colum equeous waste stream.

DEAL = Development and Analytical Laboratories.

DBP = Di-butyl phosphate.

HSH = High-solt waste.

LSW a Low-salt waste.

MBP = Mono-Butyl Phosphote.

PFP = Plutonium Finishing Plent.

PRF = Plutoniun reclemetion facility.

RMC = Remote mechanical "c" line.

SpG - Spacific gravity.

TBP = Tri-butyl phosphate.

TOC = Total organic carbon. 
DOE/RL-94-21

Table 3-2. Chemical Concentrations in Double-Shell Tank Waste.

\begin{tabular}{|c|c|c|c|c|}
\hline Constituent & $N^{*}$ & $\begin{array}{c}\text { Average } \\
\text { (moles } / L \text { ) }\end{array}$ & $\begin{array}{c}\text { Minimum } \\
\text { (moles } / L)\end{array}$ & $\begin{array}{c}\text { Maximum } \\
\text { (moles } / L \text { ) }\end{array}$ \\
\hline Aluminum & 91 & $7.8 \mathrm{E}-01$ & $1.8 \mathrm{E}-05$ & $2.9 E+00$ \\
\hline Ammonium & 31 & $9.7 \mathrm{E}-02$ & $5.0 \mathrm{E}-03$ & 4.1 E-01 \\
\hline Barium & 12 & $1.4 \mathrm{E}-03$ & $1.8 \mathrm{E}-05$ & 5.0 E-03 \\
\hline Boron & 22 & 1.9 E-02 & $5.8 E-04$ & $1.2 \mathrm{E}-01$ \\
\hline Cadmium & 9 & 4.1 E-04 & $5.9 \mathrm{E}-05$ & $1.4[-03$ \\
\hline Calcium & 40 & 5.2 E-03 & $1.9 \mathrm{E}-05$ & $1.8 E-02$ \\
\hline Carbonate & 86 & $2.4 E-01$ & 1.0 E-03 & $1.1 E+00$ \\
\hline Chloride & 74 & $1.2 \mathrm{E}-01$ & $2.6 \mathrm{E}-04$ & $9.9 \mathrm{E}-0 \mathrm{I}$ \\
\hline Chromium & 33 & $1.9 \mathrm{E}-02$ & 4.7 E-05 & $3.2 E-01$ \\
\hline Copper & 12 & 2.2 E-04 & 8.2 E-06 & $1.2 \mathrm{E}-03$ \\
\hline Fluoride & 56 & $1.0 E+00$ & $2.5 \mathrm{E}-04$ & $1.1 E+01$ \\
\hline Hydroxide & 97 & $1.7 E+00$ & $4.6 \mathrm{E}-02$ & $6.9 E+00$ \\
\hline Iron & 21 & 7.0 E-04 & 1.0 E-05 & $3.3 \mathrm{E}-02$ \\
\hline Lanthanum & 11 & $2.8 E-03$ & $2.1 E-04$ & 1.5 E-02 \\
\hline Lead & 3 & 5.7 E-03 & $6.0 \mathrm{E}-04$ & $1.5 E-02$ \\
\hline Magnesium & 30 & $4.3 E-03$ & 1.6 E-06 & 4.2 E-02 \\
\hline Manganese & 15 & $5.9 \quad E-02$ & $5.7 E-05$ & $8.3 E-01$ \\
\hline Mercury & 1 & $1.5 E-04$ & - & -- \\
\hline Molybdenum & 11 & $8.8 \mathrm{E}-04$ & $1.1 \mathrm{E}-04$ & $2.2 E-03$ \\
\hline Nicke1 & 22 & $2.5 \mathrm{E}-03$ & 4.2 E-04 & $8.8 E-03$ \\
\hline Nitrate & 99 & $1.5 E+00$ & $2.0 \mathrm{E}-03$ & $4.1 E+00$ \\
\hline Nitrite & 101 & $9.9 \mathrm{E}-01$ & 1.6 E-05 & $3.6 E+00$ \\
\hline Phosphate & 77 & 5.8 E-02 & 2.0 E-07 & $3.9 \quad E-01$ \\
\hline Phosphorus & 19 & 9.6 E-02 & $3.9 \mathrm{E}-03$ & $3.8 \mathrm{E}-01$ \\
\hline Potassium & 68 & $2.0 E-01$ & $1.1 E-04$ & $6.9 E-01$ \\
\hline silicon & 20 & 8.0 E-02 & $4.8 E-04$ & $4.7 E-01$ \\
\hline Silver & 1 & $1.8 \mathrm{E}-03$ & -- & - - \\
\hline Sodium & 79 & $5.7 E+00$ & $4.0 \mathrm{E}-04$ & $1.6 E+01$ \\
\hline Sulfate & 48 & 5.0 E-02 & $1.5 E-04$ & 8.9 E-01 \\
\hline Zine & 10 & 5.1 E-04 & $2.1 \mathrm{E}-04$ & $1.2 \mathrm{E}-03$ \\
\hline Zirconium & 16 & 5.7 E-01 & 3.4 E-04 & $1.6 E+00$ \\
\hline TOC & 91 & $1.3 E+01$ & $1.5 \mathrm{E}-02$ & $8.9 E+01$ \\
\hline
\end{tabular}


Table 3-3. Waste Generation for Various Facilities and Programs (cubic meters).

\begin{tabular}{|c|c|c|c|c|c|c|c|c|c|c|c|c|}
\hline & Plent & PUREX & $\begin{array}{l}\text { Tank } \\
\text { farme }\end{array}$ & $\begin{array}{l}\text { SST to } \\
\text { Ost } \\
\text { Pumping }\end{array}$ & $\begin{array}{l}\mathrm{NO}_{3} \\
\text { Plaht }\end{array}$ & PFP & $\stackrel{T}{T}$ & $\begin{array}{l}\text { S Plant } \\
\text { (Labora: } \\
\text { tories) }\end{array}$ & $\begin{array}{c}100 \\
\text { Area }\end{array}$ & $\begin{array}{c}300 \\
\text { Area }\end{array}$ & $\begin{array}{l}400 \\
\text { Area }\end{array}$ & Total \\
\hline FY 1990 & 2,393 & 6,882 & 1.226 & 0 & 0 & 53 & 151 & 121 & 193 & 136 & 0 & 11,155 \\
\hline FY 1991 & 1,317 & 984 & 776 & 859 & 0 & 0 & 160 & 170 & 0 & 208 & 0 & 4,454 \\
\hline fy 1992 & 435 & 363 & 155 & 458 & 0 & 136 & 250 & 106 & 0 & 132 & 30 & 2,065 \\
\hline FY 1993 & 511 & 291 & 144 & 140 & 0 & 19 & 257 & 38 & 0 & 87 & 45 & $1,532^{\circ}$ \\
\hline
\end{tabular}

In eddition to the wate categories in the table, in 1993, epproximately 1,336 cuble meters of woter was edded to DsTs. This water was used to test the uporedad 242-A Eveporator components before restart.

DST = Double-sholl tenk.

PFP = Plutoniun Finishing Plent.

sst a single-shell tenk. 
Table 3-4. Plutonium-Uranium Extraction Aging Waste Composition.

\begin{tabular}{|c|c|}
\hline Constituent & Concentration (moles/L) \\
\hline $\mathrm{Na}^{+}$ & 3.97 \\
\hline $\mathrm{OH}^{-}$ & 0.77 \\
\hline $\mathrm{AlO}_{2}^{-}$ & 0.55 \\
\hline $\mathrm{NO}_{3}^{-}+\mathrm{NO}_{2}^{-}$ & 2.02 \\
\hline $\mathrm{SO}_{4}^{-2}$ & 0.13 \\
\hline $\mathrm{F}^{-}$ & 0.13 \\
\hline $\mathrm{Fe}(\mathrm{OH})_{3}$ & 0.10 \\
\hline $\mathrm{Cr}(\mathrm{OH})_{3}$ & 0.009 \\
\hline $\mathrm{Ni}(\mathrm{OH})_{2}$ & 0.006 \\
\hline Density & $1.17-1.20 \mathrm{~g} / \mathrm{cm}^{3}$ \\
\hline
\end{tabular}


Table 3-5. Sample Analysis for Plutonium-Uranium Extraction Aging Waste Stored in Tanks 241-AZ-101 and 241-AZ-102.

\begin{tabular}{|c|c|c|c|c|}
\hline Constituent & $N *$ & $\begin{array}{l}\text { Average } \\
\text { (moles/L) }\end{array}$ & $\begin{array}{l}\text { Minimum } \\
\text { (moles/L) }\end{array}$ & $\underset{\text { Maximum }}{\text { (moles } / L)}$ \\
\hline Aluminum & 18 & $2.2 E-01$ & $2.0 E-05$ & $4.8 E-01$ \\
\hline Barium & 2 & $1.4 E-03$ & $4.0 E-10$ & $2.8 E-03$ \\
\hline Boron & 2 & 5.9 E-04 & 7.1 E-06 & $1.2 \mathrm{E}-03$ \\
\hline Cadmium & 2 & $2.9 E-04$ & $9.0 E-09$ & $5.7 E-04$ \\
\hline Calcium & 9 & $3.2 E-02$ & $3.2 E-07$ & $2.5 E-01$ \\
\hline Carbonate & 10 & $1.6 E-01$ & $6.7 E-04$ & $2.7 E-01$ \\
\hline Chloride & 4 & $2.7 E-02$ & $7.0 \mathrm{E}-03$ & $6.1 E-02$ \\
\hline Chromium & 12 & $6.3 E-03$ & $2.6 E-07$ & $1.3 E-02$ \\
\hline Copper & 4 & $1.7 E-04$ & $4.4 E-05$ & $3.5 E-04$ \\
\hline Fluoride & 8 & $1.6 E+00$ & $4.6 E-03$ & $1.3 E+01$ \\
\hline Hydroxide & 12 & $5.1 E-01$ & $7.1 E-03$ & $1.1 E+00$ \\
\hline Iron & 4 & $2.4 E-01$ & $2.4 E-07$ & $6.2 E-01$ \\
\hline Lanthanum & 1 & $1.4 E-02$ & - & - \\
\hline Lead & 2 & $3.7 E-03$ & $4.0 E-04$ & 7.0 E-03 \\
\hline Magnesium & 6 & $4.5 E-02$ & $6.9 \mathrm{E}-08$ & $2.0 \mathrm{E}-01$ \\
\hline Molybdenum & 3 & $1.6 E-03$ & $9.0 E-04$ & $3.4 E-03$ \\
\hline Nicksl & 5 & $1.7 E-02$ & $2.1 E-08$ & $8.0 E-02$ \\
\hline Nitrate & 14 & $7.1 E-01$ & $2.5 E-02$ & $1.8 E+00$ \\
\hline Nitrite & 13 & $3.3 E-01$ & $3.5 E-03$ & $7.9 E-01$ \\
\hline Phosphate & 11 & $1.4 E-01$ & $3.1 E-04$ & $8.7 E-01$ \\
\hline Phosphorus & 7 & $2.0 E-01$ & 6.4 E-07 & $8.2 E-01$ \\
\hline Potassium & 6 & $5.4 E-02$ & $3.8 E-06$ & $1.2 E-01$ \\
\hline silicon & 4 & $1.3 E-02$ & $1.7 E-05$ & $5.0 E-02$ \\
\hline Silver & 1 & $1.7 E-04$ & -- & -- \\
\hline Sodium & 16 & $3.4 E+00$ & $2.6 E-04$ & $8.5 E+00$ \\
\hline Sulfate & 9 & $9.3 E-02$ & $6.9 E-03$ & $1.6 E-01$ \\
\hline Zinc & 2 & $8.5 E-04$ & $7.0 E-09$ & $1.7 E-03$ \\
\hline Zirconium & 2 & $1.9 E-01$ & $7.5 E-08$ & $3.7 E-01$ \\
\hline TOC & 16 & $1.3 E+01$ & $5.2 E-02$ & $1.0 E+02$ \\
\hline
\end{tabular}

* Number of samples. 
Table 3-6. Estimated Mass of Nonradioactive Chemical Components of Existing Single-Shell Tank Waste after Completion of Jet Pumping.

\begin{tabular}{|c|c|c|c|}
\hline Chemtcal & $\begin{array}{l}\text { Total bulk } \\
\text { sludge }(t)\end{array}$ & $\begin{array}{l}\text { Total bulk } \\
\text { salt cake (t) }\end{array}$ & $\begin{array}{l}\text { Interstitial } \\
\text { liquid }(t)\end{array}$ \\
\hline $\mathrm{NaNO}_{3}$ & 20,000 & 110,000 & 2,500 \\
\hline $\mathrm{NaNO}_{2}$ & 3,000 & 2,300 & 1,900 \\
\hline $\mathrm{Na}_{2} \mathrm{CO}_{3}$ & 1,700 & 730 & 70 \\
\hline $\mathrm{NaOH}$ & 4,200 & 2,000 & 740 \\
\hline $\mathrm{NaAlO}_{2}$ & 950 & 1,900 & 1,500 \\
\hline $\mathrm{Na}_{3} \mathrm{PO}_{4}$ & 12,500 & 2,100 & 280 \\
\hline Cancrinite & 2,700 & -- & -- \\
\hline $\mathrm{Al}(\mathrm{OH})_{3}$ & 2,300 & -- & - \\
\hline $\mathrm{Ce}(\mathrm{OH})_{3}$ & 320 & -- & -- \\
\hline $\mathrm{Cr}(\mathrm{OH})_{3}$ & 190 & -- & -- \\
\hline $\mathrm{Cd}(\mathrm{OH})_{2}$ & 5 & - & -- \\
\hline $\mathrm{Fe}(\mathrm{OH})_{3}$ & 1,200 & -- & -- \\
\hline $\mathrm{Sr}(\mathrm{OH})_{2}$ & 50 & - & -- \\
\hline $\mathrm{BIPO}_{4}$ & 380 & -- & - \\
\hline $\mathrm{CaCO}_{3}$ & 320 & - & -- \\
\hline$F^{-}$ & 800 & -- & - \\
\hline $\mathrm{CI}^{\circ}$ & 40 & -- & -- \\
\hline $\mathrm{Hg}^{+}$ & 0.9 & -- & -- \\
\hline $\mathrm{MnO}_{2}$ & 190 & - & -- \\
\hline $\mathrm{Ni}_{2} \mathrm{Fe}(\mathrm{CN})_{6}$ & 500 & -- & -- \\
\hline $\mathrm{P}_{2} \mathrm{O}_{5} \cdot 2 \mathrm{H}_{2} \mathrm{O} \cdot 44 \mathrm{H}_{2} \mathrm{O}$ & 20 & -- & -- \\
\hline $\mathrm{ZrO}_{2} \cdot 2 \mathrm{H}_{2} \mathrm{O}$ & 430 & -- & -- \\
\hline Organic Carbon & -- & -- & 200 \\
\hline $\mathrm{H}_{2} \mathrm{O}$ & 26,000 & 14,000 & 4,800 \\
\hline Totals & 77,796 & 133,030 & 11,990 \\
\hline
\end{tabular}

NOTE: This table is from RHO-RE-ST-30P, page 2-11 (RHO 1985). $(t)=$ Metric tons.

Known silica additions are assumed to have reacted with aluminates and hydroxides to form cancrinite (assumed to be $\left.2 \mathrm{NaAlSiO}_{4} \cdot 0.52 \mathrm{NaNO}_{3} \cdot 0.68 \mathrm{H}_{2} \mathrm{O}\right)$. 
DOE/RL-94-21

Table 3-7. Hanford Site Single-Shell Tank Releases." (sheet 1 of 3)

\begin{tabular}{|l|c|c|}
\hline \multicolumn{1}{|c|}{ Tank } & Volume $\left(\mathrm{m}^{3}\right)$ & Leak Reported \\
\hline $241-\mathrm{A}-103$ & 21 & 1987 \\
\hline $241-\mathrm{A}-104$ & 9.5 & 1975 \\
\hline $241-\mathrm{A}-105$ & 19 & 1963 \\
\hline $241-\mathrm{AX}-102$ & 11 & 1988 \\
\hline $241-\mathrm{B}-107$ & 30 & 1980 \\
\hline $241-\mathrm{B}-110$ & 38 & 1981 \\
\hline $241-\mathrm{B}-201$ & 4.5 & 1980 \\
\hline $241-\mathrm{B}-203$ & 1.1 & 1983 \\
\hline $241-\mathrm{BX}-102$ & 265 & 1971 \\
\hline $241-\mathrm{BX}-108$ & 9.5 & 1974 \\
\hline $241-\mathrm{BY}-103$ & $<19$ & 1973 \\
\hline $241-\mathrm{BY}-108$ & $<19$ & 1972 \\
\hline $241-\mathrm{C}-101$ & 76 & 1980 \\
\hline $241-\mathrm{C}-201$ & 2.1 & 1988 \\
\hline $241-\mathrm{C}-202$ & 1.7 & 1988 \\
\hline $241-\mathrm{C}-203$ & 1.5 & 1984 \\
\hline $241-\mathrm{C}-204$ & 1.3 & 1988 \\
\hline $241-\mathrm{SX}-104$ & 23 & 1988 \\
\hline $241-\mathrm{SX}-107$ & 19 & 1964 \\
\hline $241-\mathrm{SX}-108$ & 9.1 & 1962 \\
\hline $241-\mathrm{SX}-109$ & 19 & 1965 \\
\hline $241-\mathrm{SX}-110$ & 21 & 1976 \\
\hline $241-\mathrm{SX}-111$ & 7.6 & 1974 \\
\hline $241-\mathrm{SX}-112$ & 114 & 1969 \\
\hline $241-\mathrm{SX}-113$ & 57 & 1962 \\
\hline $241-\mathrm{SX}-115$ & 189 & 1965 \\
\hline $241-\mathrm{T}-101$ & $<28$ & 1992 \\
\hline $241-\mathrm{T}-106$ & 436 & 1973 \\
\hline $241-\mathrm{T}-108$ & 3.8 & 1974 \\
\hline $241-\mathrm{T}-111$ & 3.8 & 1984 \\
\hline & & \\
\hline
\end{tabular}


Table 3-7. Hanford Site Single-Shell Tank Releases. (sheet 2 of 3 )

\begin{tabular}{|c|c|c|}
\hline Tank & Volume $\left(\mathrm{m}^{3}\right)$ & Leak Reported \\
\hline $241-T-107$ & 9.5 & 1984 \\
\hline $241-T Y-101$ & $<3.8$ & 1973 \\
\hline $241-T Y-103$ & 11 & 1973 \\
\hline $241-T Y-104$ & 5.3 & 1981 \\
\hline $241-T Y-105$ & 133 & 1960 \\
\hline $241-T Y-106$ & 76 & 1959 \\
\hline $241-U-101$ & 114 & 1959 \\
\hline $241-U-104$ & 208 & 1961 \\
\hline $241-U-110$ & 31 & 1975 \\
\hline $241-U-112$ & 32 & 1980 \\
\hline 241-B-204 & 1.5 & 1984 \\
\hline 241-BY-107 & 57 & 1984 \\
\hline $241-C-111$ & 21 & 1968 \\
\hline $241-S-104$ & 91 & 1968 \\
\hline $241-T-103$ & $<3.8$ & 1974 \\
\hline $241-T-109$ & $<3.8$ & 1974 \\
\hline 241-B-112 & 7.6 & 1978 \\
\hline $241-C-110$ & 7.6 & 1984 \\
\hline $241-A X-104^{b}$ & -- & 1977 \\
\hline $241-B-101^{b}$ & -- & 1974 \\
\hline $241-B-103^{b}$ & -- & 1978 \\
\hline $241-B-105^{b}$ & -- & 1978 \\
\hline $241-B-111^{b}$ & - & 1978 \\
\hline $241-B X-101^{b}$ & -- & 1972 \\
\hline $241-B X-110^{b}$ & -- & 1976 \\
\hline $241-B X-111^{b}$ & -- & 1984,1993 \\
\hline $241-B Y-105^{b}$ & -- & 1984 \\
\hline $241-B Y-106^{b}$ & - & 1984 \\
\hline $241-5 x-114^{b}$ & -- & 1972 \\
\hline
\end{tabular}


Table 3-7. Hanford Site Single-Shell Tank Releases." (sheet 3 of 3 )

\begin{tabular}{|l|c|c|}
\hline \multicolumn{1}{|c|}{ Tank } & Volume $\left(\mathrm{m}^{3}\right)$ & Leak Reported \\
\hline $241-\mathrm{TX}-107^{\mathrm{b}}$ & -- & 1984 \\
\hline $241-\mathrm{TX}-105^{\mathrm{b}}$ & -- & 1977 \\
\hline $241-\mathrm{TX}-110^{\mathrm{b}}$ & -- & 1977 \\
\hline $241-\mathrm{TX}-113^{\mathrm{b}}$ & -- & 1974 \\
\hline $241-\mathrm{TX}-114^{\mathrm{b}}$ & -- & 1974 \\
\hline $241-\mathrm{TX}-115^{\mathrm{b}}$ & -- & 1977 \\
\hline $241-\mathrm{TX}-116^{\mathrm{b}}$ & -- & 1977 \\
\hline $241-\mathrm{TX}-117^{\mathrm{b}}$ & -- & 1977 \\
\hline $\begin{array}{l}\text { Total esstimated leakage volume from } 67 \text { tanks: } \\
2,840 \mathrm{~m}^{3} .\end{array}$ \\
\hline
\end{tabular}

After some tanks were declared to be leaking, cooling water may have been added to aid evaporative cooling. It is believed that some of this water did not evaporate and, therefore, went into the ground. As of October 1990, estimates ranged from 190 to 3,000 cubic meters. The past practice was to exclude the cooling water from the leak volume estimate. The volumes provided and date of initial release are the subject of continued evaluation and refinement and may be revised for improved accuracy as a result of these evaluations. In addition, documents show that from 1946 to $1966,456,725$ cubic meters $(120,661,000$ gallons $)$ of liquid wastes were intentionally discharged from SSTs at the Hanford Site directly to the gruund on the 200 Area plateau (WHC 1991C). The majority of this waste was discharged from 1946 to 1958 as a result of the early plutonium and uranium recovery processes conducted in the 221-B Facility (B Plant), 221-T Facility (T Plant), and the 221-U Facility (U Plant). In addition, from 1960 to 1966 laboratory wastes from the 300 Area and equipment decontamination wastes from the 200 West Area were routed through SSTs before discharge to the ground. No wastes have been discharged intentionally to the ground from SSTs since 1966, and no wastes have ever been discharged directly to the ground from the newer DSTs located at the Hanford Site.

Individual release volumes for these tanks have not been determined. The total volume release from these tanks is estimated to be 570 cubic meters.

SST = Single-shell tank. 
Table 3-8. Analytes Detected in 242-A Evaporator Process Condensate. (sheet 1 of 3 )

\begin{tabular}{|c|c|c|}
\hline Analyte & $\begin{array}{l}\text { Number of samples } \\
\text { containing analyte }\end{array}$ & $\begin{array}{c}\text { Average concentration } \\
(\mathrm{ppb})\end{array}$ \\
\hline Total organic carbon & 31 & $2.6 E+04$ \\
\hline \multicolumn{3}{|c|}{ INORGANIC CATIONS } \\
\hline Aluminum & 29 & $8.4 E+02$ \\
\hline Ammonium & 31 & $4.1 E+05$ \\
\hline Barium & 4 & $6.8 E+00$ \\
\hline Boron & 1 & $1.3 E+01$ \\
\hline Cadmium & 1 & $5.0 E+00$ \\
\hline Calcium & 31 & $2.7 E+03$ \\
\hline Copper & 5 & $2.6 E+01$ \\
\hline Iron & 9 & $6.3 E+01$ \\
\hline Magnesium & 14 & $5.0 E+02$ \\
\hline Manganese & 1 & $5.0 E+00$ \\
\hline Mercury & 24 & $3.0 \mathrm{E}-01$ \\
\hline Nickel & 5 & $1.4 E+01$ \\
\hline Potassium & 30 & $2.6 E+03$ \\
\hline Silicon & 4 & $6.8 E+03$ \\
\hline Sodium & 25 & $3.3 E+03$ \\
\hline Vanadium & 4 & $6.3 E+00$ \\
\hline Zinc & 15 & $1.3 E+01$ \\
\hline \multicolumn{3}{|c|}{ INORGANIC ANIONS } \\
\hline Chloride & 7 & $1.0 E+03$ \\
\hline Fluoride (ion chromatogram) & 1 & $2.1 E+03$ \\
\hline $\begin{array}{l}\text { Fluoride (ion-specific } \\
\text { electrode) }\end{array}$ & 7 & $4.0 E+01$ \\
\hline Nitrate & 4 & $2.8 E+03$ \\
\hline Sulfate & 17 & $2.6 E+03$ \\
\hline Sulfide & 2 & $3.6 E+04$ \\
\hline
\end{tabular}


Table 3-8. Analytes Detected in 242-A Evaporator Process Condensate. (sheet 2 of 3 )

\begin{tabular}{|c|c|c|}
\hline Analyte & $\begin{array}{l}\text { Number of samples } \\
\text { containing analyte }\end{array}$ & $\begin{array}{c}\text { Average concentration } \\
(\mathrm{ppb})\end{array}$ \\
\hline \multicolumn{3}{|c|}{ ACIDS } \\
\hline Caproic acid & 1 & $7.0 \mathrm{E}+01$ \\
\hline \multicolumn{3}{|c|}{ PARAFFINS } \\
\hline 2-Methylnonane & 2 & $1.6 E+01$ \\
\hline Dodecane & 2 & $4.3 E+01$ \\
\hline Heptadecane & 1 & $1.8 E+01$ \\
\hline Hexadecane & 1 & $1.7 E+01$ \\
\hline Pentadecane & 1 & $2.0 E+01$ \\
\hline Tetradecane & 23 & $7.6 E+01$ \\
\hline Tridecane & 23 & $7.0 \mathrm{E}+01$ \\
\hline \multicolumn{3}{|c|}{ ALCOHOLS } \\
\hline 2-Propanol & 10 & $2.2 E+01$ \\
\hline Butyl alcohol & 30 & $9.8 E+03$ \\
\hline Ethyl alcohol & 1 & $2.0 E+00$ \\
\hline \multicolumn{3}{|c|}{ ALDEHYDES } \\
\hline Butylaldehyde & 14 & $5.6 E+01$ \\
\hline \multicolumn{3}{|c|}{ KETONES } \\
\hline Acetone & 36 & $9.8 E+02$ \\
\hline Methyl ethyl ketone & 25 & $5.1 E+01$ \\
\hline Methyl n-butyl ketone & 16 & $1.3 E+01$ \\
\hline Methyl n-propyl ketone & 8 & $9.3 E+00$ \\
\hline Hexone & 10 & $1.1 E+01$ \\
\hline \multicolumn{3}{|c|}{ CYCLICS } \\
\hline Benzaldehyde & 1 & $2.3 E+01$ \\
\hline Benzyl alcohol & 9 & $1.3 E+01$ \\
\hline Phenol & 1 & $3.3 E+01$ \\
\hline Tetrahydrofuran & 24 & $3.7 E+01$ \\
\hline \multicolumn{3}{|c|}{ ESTERS } \\
\hline Tributyl phosphate & 31 & $3.9 E+03$ \\
\hline
\end{tabular}


Table 3-8. Analytes Detected in 242-A Evaporator Process Condensate. (sheet 3 of 3 )

\begin{tabular}{|l|c|c|}
\hline \multicolumn{1}{|c|}{ Analyte } & $\begin{array}{c}\text { Number of samples } \\
\text { containing analyte }\end{array}$ & $\begin{array}{c}\text { Average concentration } \\
(\mathrm{ppb})\end{array}$ \\
\hline \multicolumn{3}{|c|}{ NITROGEN-CONTAINING ORGANICS } \\
\hline 3,5-Dimethylpridine & 3 & $2.1 \mathrm{E}+01$ \\
\hline Dimethylnitrosamine & 1 & $5.7 \mathrm{E}+01$ \\
\hline Pyridine & 1 & $5.5 \mathrm{E}+02$ \\
\hline & 25 & $3.8 \mathrm{E}+02$ \\
\hline 2-Butoxyethanol & 2 & $1.9 \mathrm{E}+01$ \\
\hline Butoxydiglycol & 21 & $2.8 \mathrm{E}+02$ \\
\hline Butoxyglycol & 1 & $3.5 \mathrm{E}+01$ \\
\hline Butoxytriethylene glycol & 4 & $9.9 \mathrm{E}+01$ \\
\hline Ethoxytriethylene glycol & 2 & $4.0 \mathrm{E}+01$ \\
\hline Methoxydiglycol & 2 & $2.2 \mathrm{E}+02$ \\
\hline Methoxytriglycol & 1 & $9.0 \mathrm{E}+01$ \\
\hline Triglyme & \multicolumn{2}{c|}{} \\
\hline & 21 & $1.6 \mathrm{E}+01$ \\
\hline Strontium & RADIONUCLIDES \\
\hline Uranium & $4.4 \mathrm{E}-01$ \\
\hline
\end{tabular}


Table 3-9. Analytes Reported in Plutonium-Uranium Extraction Plant Ammonia Scrubber Discharge.

\begin{tabular}{|l|c|c|}
\hline \multicolumn{1}{|c|}{ Constituent } & $\mathrm{N}^{4}$ & Average concentration \\
\hline Calcium & 4 & $6.80 \mathrm{E}+01$ \\
\hline Chloride & 4 & $1.17 \mathrm{E}+03$ \\
\hline Chromium & 4 & $1.06 \mathrm{E}+01$ \\
\hline Magnesium & 1 & $2.10 \mathrm{E}+01$ \\
\hline Nickel & 4 & $1.02 \mathrm{E}+01$ \\
\hline Nitrate & 4 & $5.50 \mathrm{E}+02$ \\
\hline Sodium & 4 & $2.79 \mathrm{E}+02$ \\
\hline Uranium & 4 & $3.91 \mathrm{E}-01$ \\
\hline Zinc & 4 & $3.50 \mathrm{E}+01$ \\
\hline Ammonia & 4 & $3.66 \mathrm{E}+05$ \\
\hline 1-Butanol & 1 & $1.20 \mathrm{E}+01$ \\
\hline Alpha Activity (pCi/L) & 4 & $3.01 \mathrm{E}+01$ \\
\hline Beta Activity (pCi/L) & 4 & $3.99 \mathrm{E}+04$ \\
\hline Conductivity (uS) & 4 & $1.79 \mathrm{E}+02$ \\
\hline pH (dimensionless) & 4 & $9.35 \mathrm{E}+00$ \\
\hline Temperature ( $\left.{ }^{\circ} \mathrm{C}\right)$ & 4 & $3.24 \mathrm{E}+01$ \\
\hline TOC & 4 & $2.16 \mathrm{E}+03$ \\
\hline
\end{tabular}

" $N$ is the number of samples in which the analyte was detected. The average concentrations do not reflect "less than" values. It is the sum of the detected values divided by $N$.

Units are parts per billion unless otherwise stated. This ammonia scrubber discharge was sent to cribs.

TOC = Total organic hydrocarbon. 
Table 3-10. Analyses for Plutonium-Uranium Extraction Plant Ammonia Scrubber Feed Stored in Double-Shell Tanks.

\begin{tabular}{|l|c|}
\hline \multicolumn{1}{|c|}{ Analyte } & Average Concentration \\
\hline Sodium nitrite & $0.04 \mathrm{M}$ \\
\hline Ammonium hydroxide & $0.09 \mathrm{M}$ \\
\hline Fluoride & $2.6 \times 10^{-4}$ \\
\hline Hydroxide ion & 0.02 \\
\hline $\mathrm{pH}$ & $>12.5$ \\
\hline Total alpha & $0.11 \mu \mathrm{Ci} / \mathrm{L}$ \\
\hline
\end{tabular}


Table 3-11. Analytes Reported in the Plutonium-Uranium Extraction Plant Process Condensate.

\begin{tabular}{|c|c|c|}
\hline Constituent & $N *$ & Average concentration (ppb) \\
\hline Boron & 5 & $1.64 E+01$ \\
\hline Calcium & 5 & $5.02 E+01$ \\
\hline Cyanide & 5 & $3.57 E+01$ \\
\hline Fluoride & 5 & $8.60 E+02$ \\
\hline Mercury & 5 & $9.66 E-01$ \\
\hline Nitrate & 5 & $5.56 t+04$ \\
\hline Nitrite & 5 & $4.93 E+04$ \\
\hline Potassium & 5 & $5.08 E+02$ \\
\hline Silicon & 5 & $2.19 E+02$ \\
\hline Sodium & 5 & $1.29 E+04$ \\
\hline Acetone & 4 & $5.75 E+01$ \\
\hline Ammonia & 5 & $5.32 E+01$ \\
\hline 1-Butanol & 3 & $1.90 E+01$ \\
\hline 2-Butanone & 4 & $2.85 E+01$ \\
\hline Butylated hydroxy toluene & 1 & $1.00 E+02$ \\
\hline Dibutylphosphate & 4 & $1.74 E+04$ \\
\hline Dodecane & 7 & $9.14 E+03$ \\
\hline Tetradecane & 8 & $2.10 E+04$ \\
\hline Tetrahydrofuran & 4 & $7.45 \mathrm{E}+01$ \\
\hline Tributylphosphate & 8 & $7.78 E+04$ \\
\hline Tridecane & 8 & $3.28 E+04$ \\
\hline Undecane & 1 & $1.20 E+02$ \\
\hline Unknown aliphatic HC & 2 & $1.19 E+03$ \\
\hline Unknown ester & 4 & $5.24 E+02$ \\
\hline Unknown ester & 3 & $3.07 E+01$ \\
\hline Unknown hydrocarbon & 2 & $1.55 E+04$ \\
\hline Ignitability $\left({ }^{\circ} \mathrm{F}\right)$ & 5 & $2.08 E+02$ \\
\hline pH (dimensionless) & 4 & $3.04 E+00$ \\
\hline Temperature $\left({ }^{\circ} \mathrm{C}\right)$ & 3 & $4.66 E+01$ \\
\hline TOC & 5 & $1.06 E+05$ \\
\hline TOX (as C1) & 5 & $4.80 E+01$ \\
\hline
\end{tabular}

* $N$ is the number of samples in which the analyte was detected. The average concentrations do not reflect "less than" values. This analysis is for waste discharged to cribs.

TOC = Total organic hydrocarbon.

TOX = Total organic halide. 
Table 3-12. Analyses of Hexone Waste.

Analysis Before Distillation in 1990.

\begin{tabular}{|l|c|c|c|}
\hline \multirow{2}{*}{ Compound } & \multicolumn{3}{|c|}{ Concentration (Weight Percent) } \\
\cline { 2 - 4 } & Tank 276-S-141 & \multicolumn{2}{c|}{ Tank 276-S-142 } \\
\cline { 2 - 4 } & & Organic Phase & Aqueous Phase \\
\hline Hexone & 99.0 & 65.2 & 1.0 \\
\hline N-alkanes $\left(\mathrm{nC}_{10}-\mathrm{nC}_{15}\right)$ & $\mathrm{NC}$ & 14.2 & ND \\
\hline N-tributyl phosphate & ND & 8.4 & ND \\
\hline Water & 1.0 & 1.0 & 99.0 \\
\hline $\begin{array}{l}\text { Mono- and di-butyl } \\
\text { phosphates, and } n-a l k a n e s \\
\text { out of the } \mathrm{C}_{10}-\mathrm{C}_{15} \text { range }\end{array}$ & ND & 12.2 & ND \\
\hline Total & & & 100.0 \\
\hline
\end{tabular}

ND = Not detected.

Analysis of Sludge/Tar Residual Composition for

Tanks 276-S-141 (250.Gallons) and

Tanks 276-S-142 (250 Gallons).

\begin{tabular}{|c|c|c|c|}
\hline Radionuclides & $\mathrm{nCi} / \mathrm{g}$ & Metals (TCLP) & $\mu \mathrm{g} / \mathrm{g}$ \\
\hline${ }^{241} \mathrm{Am}$ & 32.3 & $\mathrm{Ba}$ & 0.8 \\
\hline${ }^{155} \mathrm{Eu}$ & 0.4 & $\mathrm{Cd}$ & 0.6 \\
\hline${ }^{154} \mathrm{Eu}$ & 3.8 & $\mathrm{Cr}$ & 5.1 \\
\hline${ }^{137} \mathrm{Cs}$ & 2.3 & $\mathrm{~Pb}$ & 7.7 \\
\hline${ }^{60} \mathrm{Co}$ & 0.003 & $\mathrm{Ag}$ & $<0.5$ \\
\hline${ }^{175} \mathrm{Sb}$ & 0.8 & $\mathrm{As}$ & 0.4 \\
\hline Total alpha & 36.0 & $\mathrm{Se}$ & $<0.1$ \\
\hline Total beta & 38.5 & $\mathrm{Hg}$ & $<0.2$ \\
\hline${ }^{239 / 40} \mathrm{Pu}$ & 7.4 & -- & - \\
\hline
\end{tabular}


Table 3-13. Routine Wastes Discharged to 183-H Solar Evaporator Basins.

\begin{tabular}{|l|c|}
\hline \multicolumn{1}{|c|}{ Constituent } & Amount \\
\hline Uranium & $1,988 \mathrm{~kg}$ \\
\hline Chromium & $744 \mathrm{~kg}$ \\
\hline Manganese & $1,411 \mathrm{~kg}$ \\
\hline Copper & $197,948 \mathrm{~kg}$ \\
\hline Nitrate ion & $1,371,391 \mathrm{~kg}$ \\
\hline Sulfate ion & $341,646 \mathrm{~kg}$ \\
\hline Ammonium ion & $1,760 \mathrm{~kg}$ \\
\hline Fluoride ion & $88,360 \mathrm{~kg}$ \\
\hline Average pH & 9.8 \\
\hline
\end{tabular}

$$
\text { (Total volume }=9,623 \mathrm{~m}^{3} \text { ) }
$$


Table 3-14. Plutonium-Uranium Extraction Plant Storage Inventories. (sheet 1 of 2)

Plutonium-Uranium Extraction Plant Tunnels Inventory.

\begin{tabular}{|l|c|c|c|c|}
\hline $\begin{array}{c}\text { Date } \\
\text { transferred } \\
\text { to tunnel }\end{array}$ & $\begin{array}{c}\text { Tunnel } \\
\text { number }\end{array}$ & $\begin{array}{c}\text { Mass (Kg) of } \\
\text { lead transferred }\end{array}$ & $\begin{array}{c}\text { Mass (Kg) } \\
\text { of mercury } \\
\text { transferred }\end{array}$ & $\begin{array}{c}\text { Mass (Kg) of } \\
\text { silver nitrate } \\
\text { transferred }\end{array}$ \\
\hline $06-60$ & 1 & 113 & -- & -- \\
\hline $12-24-60$ & 1 & 113 & -- & -- \\
\hline $12-22-71$ & 2 & -- & 45 & 624 \\
\hline $12-26-71$ & 2 & -- & -- & -- \\
\hline $09-30-72$ & 2 & -- & 45 & -- \\
\hline $01-18-86$ & 2 & -- & 38 & -- \\
\hline $11-18-87$ & 2 & 2,530 & -- & 113 \\
\hline $06-13-88$ & 2 & 227 & -- & 737 \\
\hline $06-13-88$ & 2 & -- & -- & -129 \\
\hline Total & N/A & 2,983 & 0 & -263 \\
\hline
\end{tabular}

Note: $2983 \mathrm{~kg}$ of lead has a volume of $0.263 \mathrm{~m}^{3}$.

$129 \mathrm{Kg}$ of mercury has a volume of $0.0095 \mathrm{~m}^{3}$ at $23{ }^{\circ} \mathrm{C}$.

$737 \mathrm{Kg}$ of silver nitrate has a volume of $0.17 \mathrm{~m}^{3}$. $N / A=$ Not applicable. 
Table 3-14. Plutonium-Uranium Extraction P1ant Storage Inventories. (sheet 2 of 2)

Plutonium-Uranium Extraction Plant Containment Building Storage Inventory.

\begin{tabular}{|l|c|c|}
\hline Date transferred & $\begin{array}{c}\text { Lead mass } \\
(\mathrm{Kg})\end{array}$ & $\begin{array}{c}\text { Cadmi um mass } \\
(\mathrm{Kg})\end{array}$ \\
\hline $12-07-87$ & 23.8 & -- \\
\hline $12-14-87$ & 114.8 & -- \\
\hline $02-03-88$ & 66.2 & -- \\
\hline $02-20-88$ & 34.0 & -- \\
\hline $04-22-88$ & 113.4 & -- \\
\hline $10-12-88$ & 9.1 & -- \\
\hline $12-15-88$ & 56.2 & -- \\
\hline $07-15-89$ & 34.0 & -- \\
\hline $07-16-89$ & 29.9 & -- \\
\hline $07-17-89$ & 27.2 & -- \\
\hline $08-13-89$ & 201.9 & -- \\
\hline $08-13-89$ & 201.9 & -- \\
\hline $01-15-90$ & 267.6 & -- \\
\hline $06-22-90$ & 91.2 & -- \\
\hline $06-22-90$ & 582.4 & -- \\
\hline $06-22-90$ & $1,301.8$ & -- \\
\hline $06-22-90$ & -- & 5.9 \\
\hline $10-27-90$ & 70.3 & -- \\
\hline Tota1 & $3,225.8$ & 5.9 \\
\hline & & 0.8 \\
\hline
\end{tabular}

Note: $3,225.8 \mathrm{~kg}$ of lead has a volume of $0.284 \mathrm{~m}^{3}$. $5.9 \mathrm{Kg}$ of cadmium has a volume of $0.0295 \mathrm{~m}^{3}$. 


\section{DOE/RL-94-21}

Table 3-15. Projected Generation of Solid Waste Operations Complex Stored Low-Level and Transuranic Waste.

\begin{tabular}{|c|c|}
\hline Year & Total (cubic meters) \\
\hline 1994 & 4,273 \\
\hline 1995 & 3,502 \\
\hline 1996 & 3,205 \\
\hline 1997 & 2,877 \\
\hline 1998 & 2,682 \\
\hline 1999 & 2,941 \\
\hline 2000 & 4,031 \\
\hline 2001 & 3,684 \\
\hline 2002 & 3,452 \\
\hline 2003 & 2,662 \\
\hline 2004 & 7,025 \\
\hline 2005 & 10,652 \\
\hline 2006 & 11,243 \\
\hline 2007 & 3,428 \\
\hline 2008 & 3,502 \\
\hline 2009 & 3,289 \\
\hline 2010 & 2,930 \\
\hline 2011 & 2,059 \\
\hline 2012 & 2,525 \\
\hline 2013 & 2,006 \\
\hline 2014 & 2,026 \\
\hline 2015 & 2,309 \\
\hline 2016 & 2,481 \\
\hline 2017 & 2,185 \\
\hline 2018 & 1,983 \\
\hline 2019 & 2,002 \\
\hline 2020 & 2,110 \\
\hline 2021 & 2,081 \\
\hline 2022 & 1,970 \\
\hline Total & 101,115 \\
\hline
\end{tabular}


Table 3-16. Designation of 303-K Facility Stored Waste (December 31, 1993).

Wasie description

Organic Constituents

Perchloroethylene

Spent halogenated solvent

$1,1,1$-trichlorethane

Spent halogenated solvent

Organic Degradation Products

1,1-dichloroethylene

Spent halogenated solvent

cis-1,2-dichloroethylene

Spent halogenated solvent

trans-1,2-dichoroethylene

Spent halogenated solvent

\section{Inorganic Constituents}

5\% Beryll1um/Zircaloy-2*

Metal alloy

Cadmium ion

sludge

Chromium

STudge

Zircaloy-2*

Metal alloy

Lead

Lead metal and sludge

Comprises zirconium with 1.2-1.7\% tin, 0.07-0.2\% iron, 0.05-0.15\% chromium, and 0.03-0.08\% nickel. 

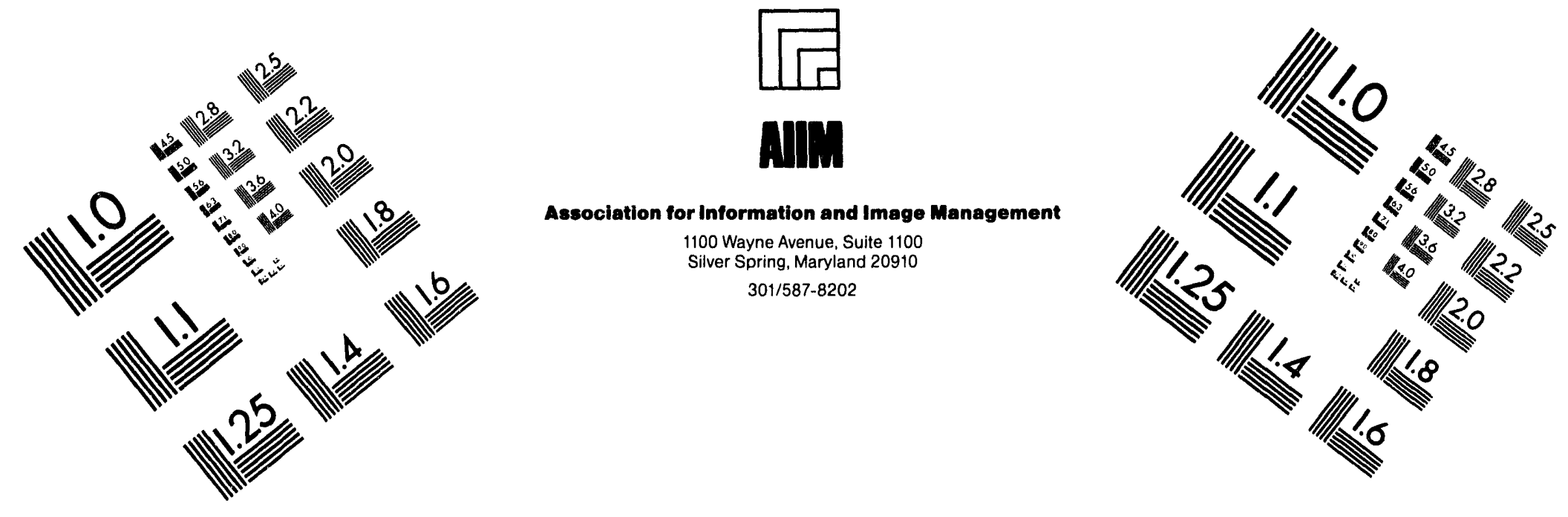

\section{Centimeter}

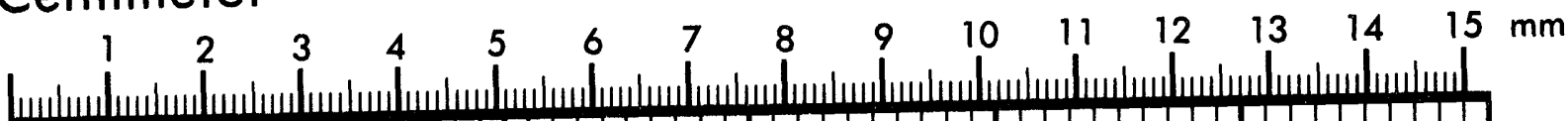

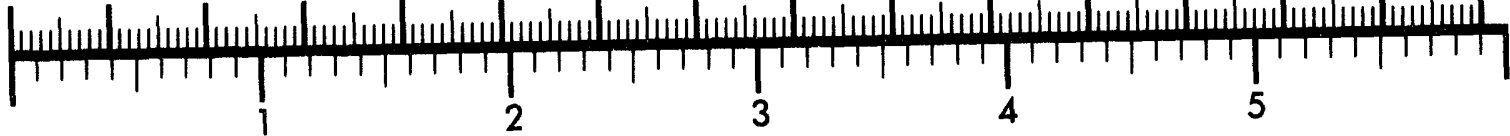
Inches
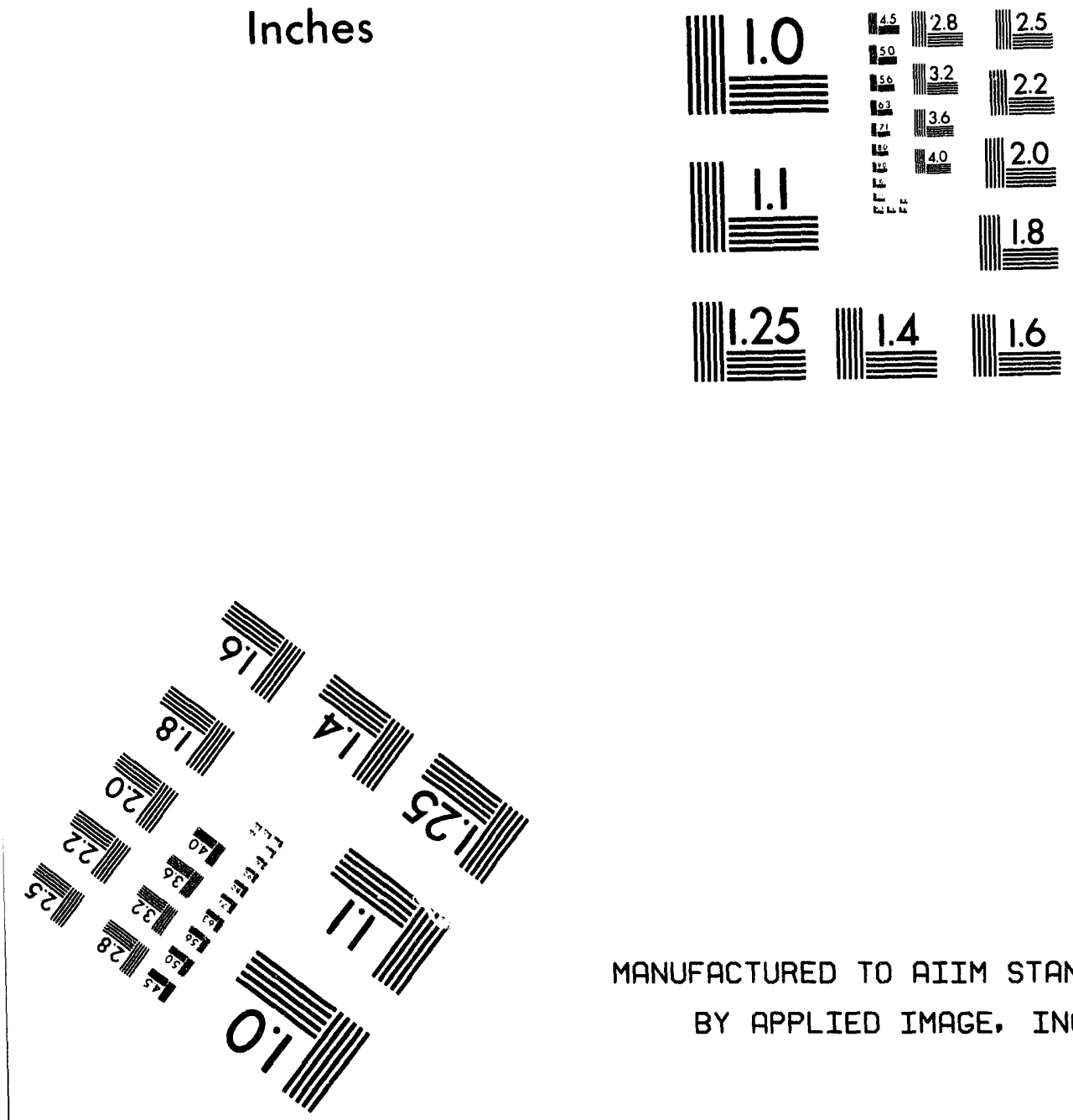

MANUFACTURED TO AIIM STANDARDS BY APPLIED IMAGE. INC.

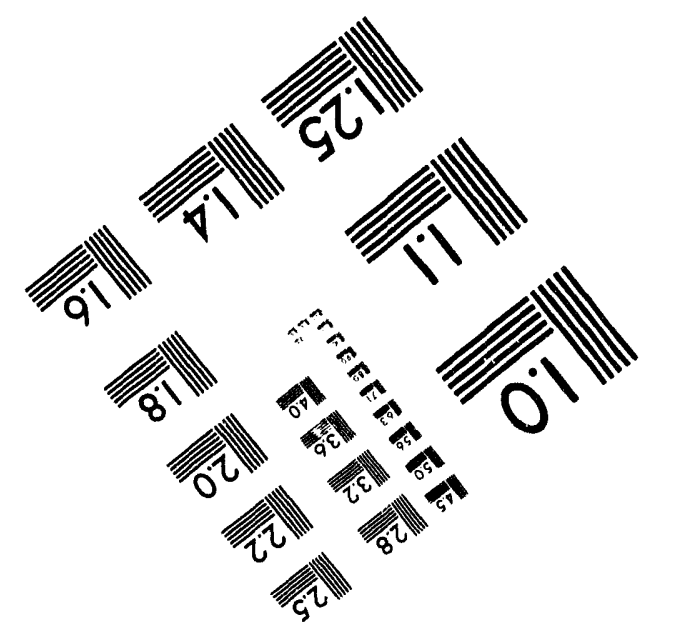



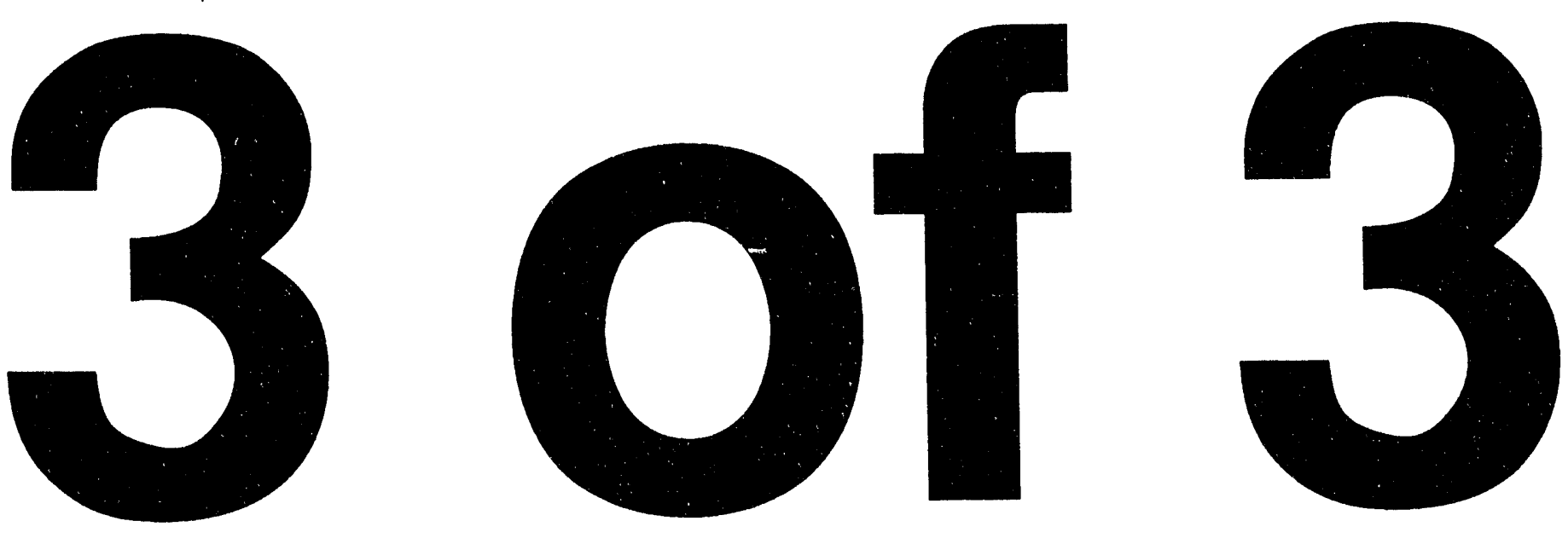


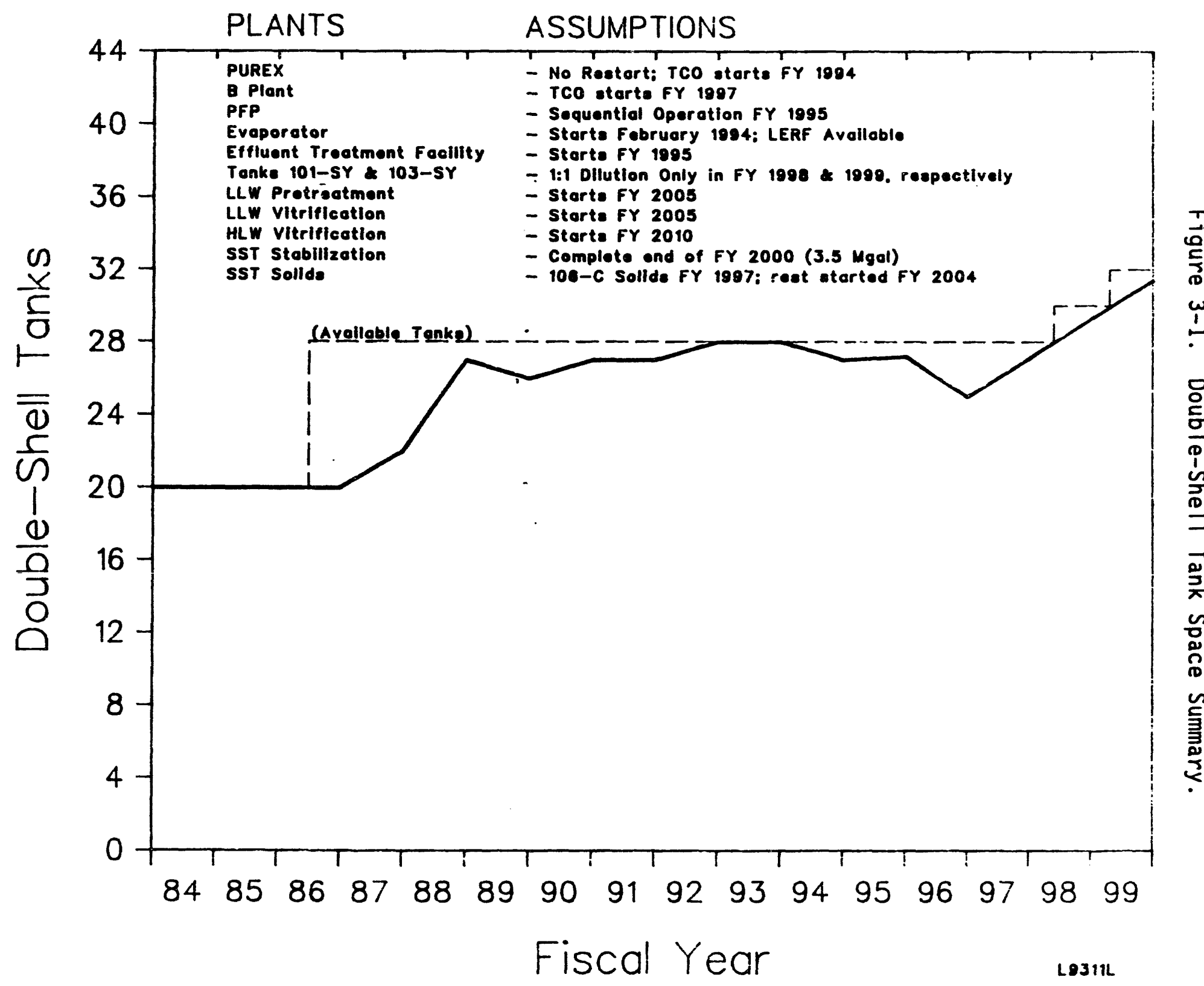


Figure 3-2. Tank Waste Remediation System Treatment Block Diagram.

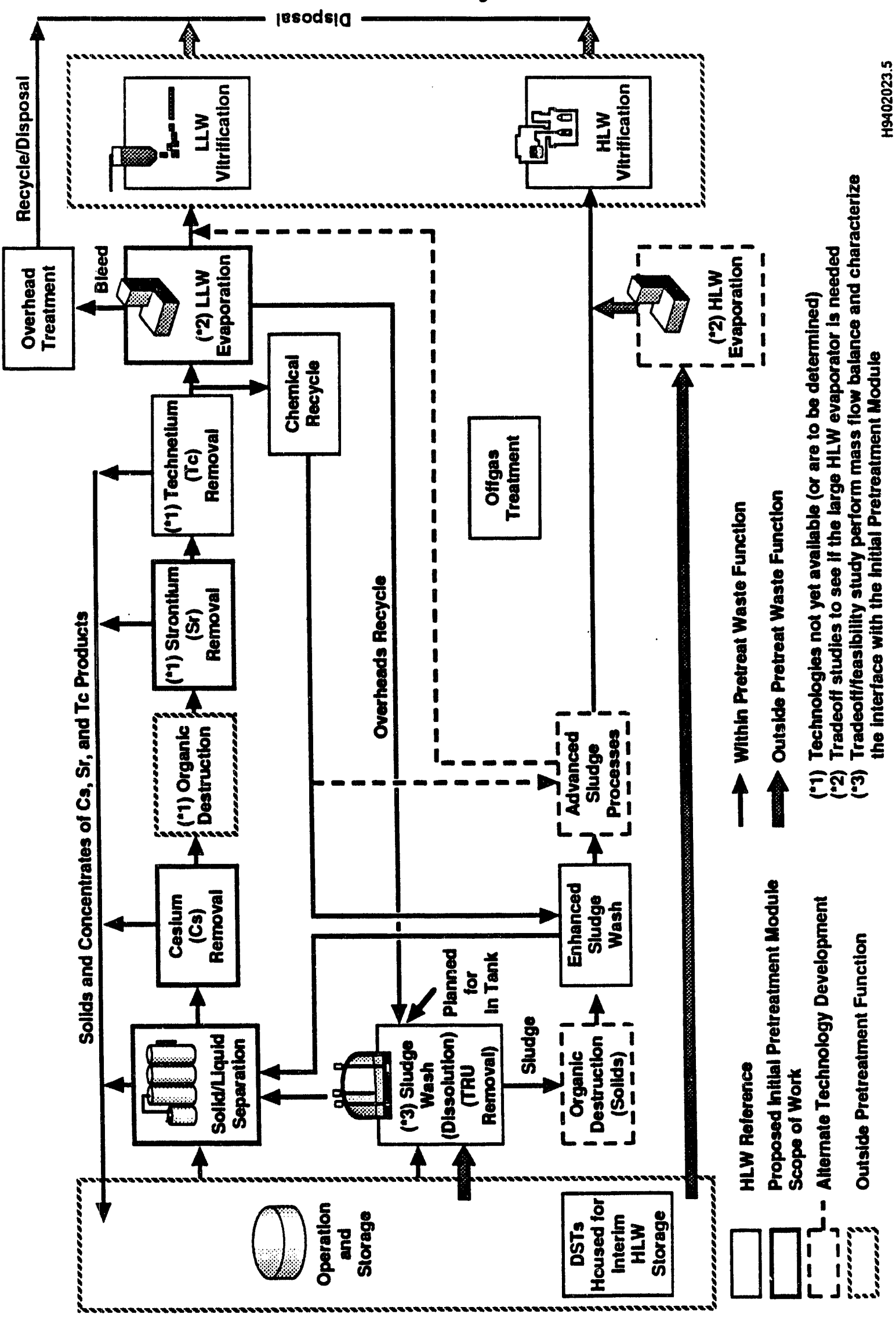


Figure 3-3. PUREX Aging Waste Transfers to Aging Waste Storage.

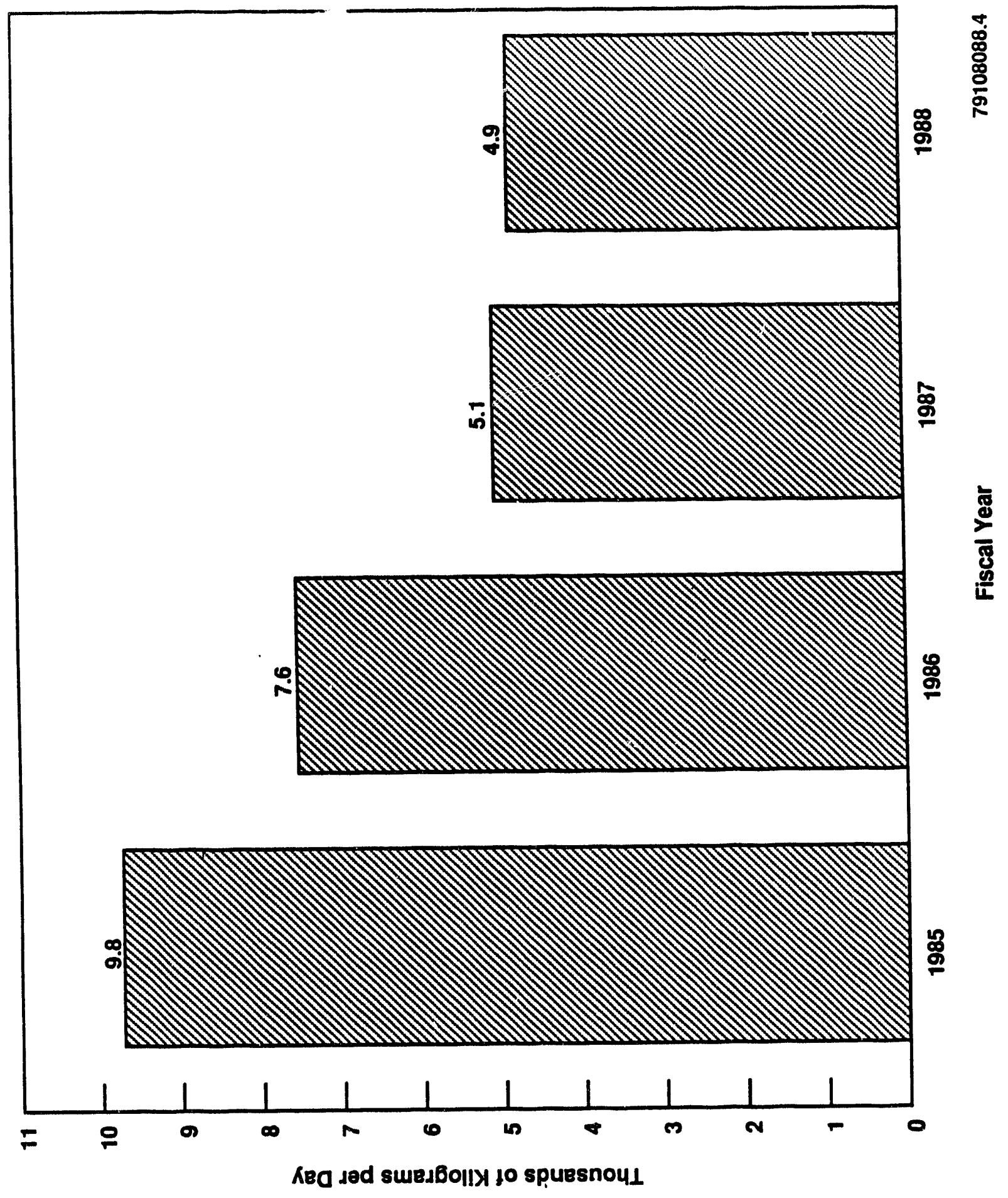


Figure 3-4. Relative Proportions of Supernatant, Sludge, and Salt Cake in Single-Shell Waste.

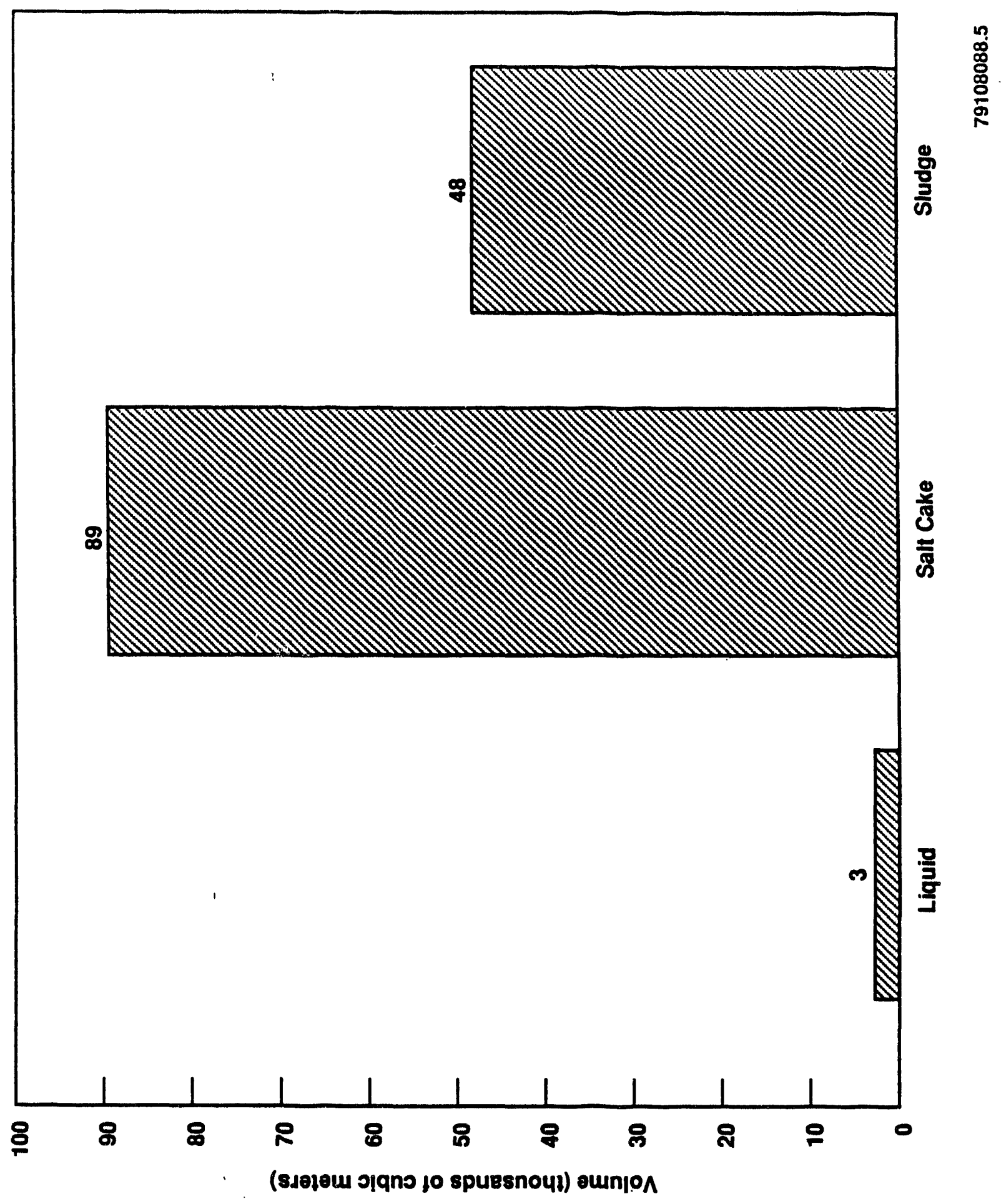

F $3-4.1$ 
DOE/RL-94-21

Figure 3-5. Single-Shell Tank Waste Inventory by Tank Farm.

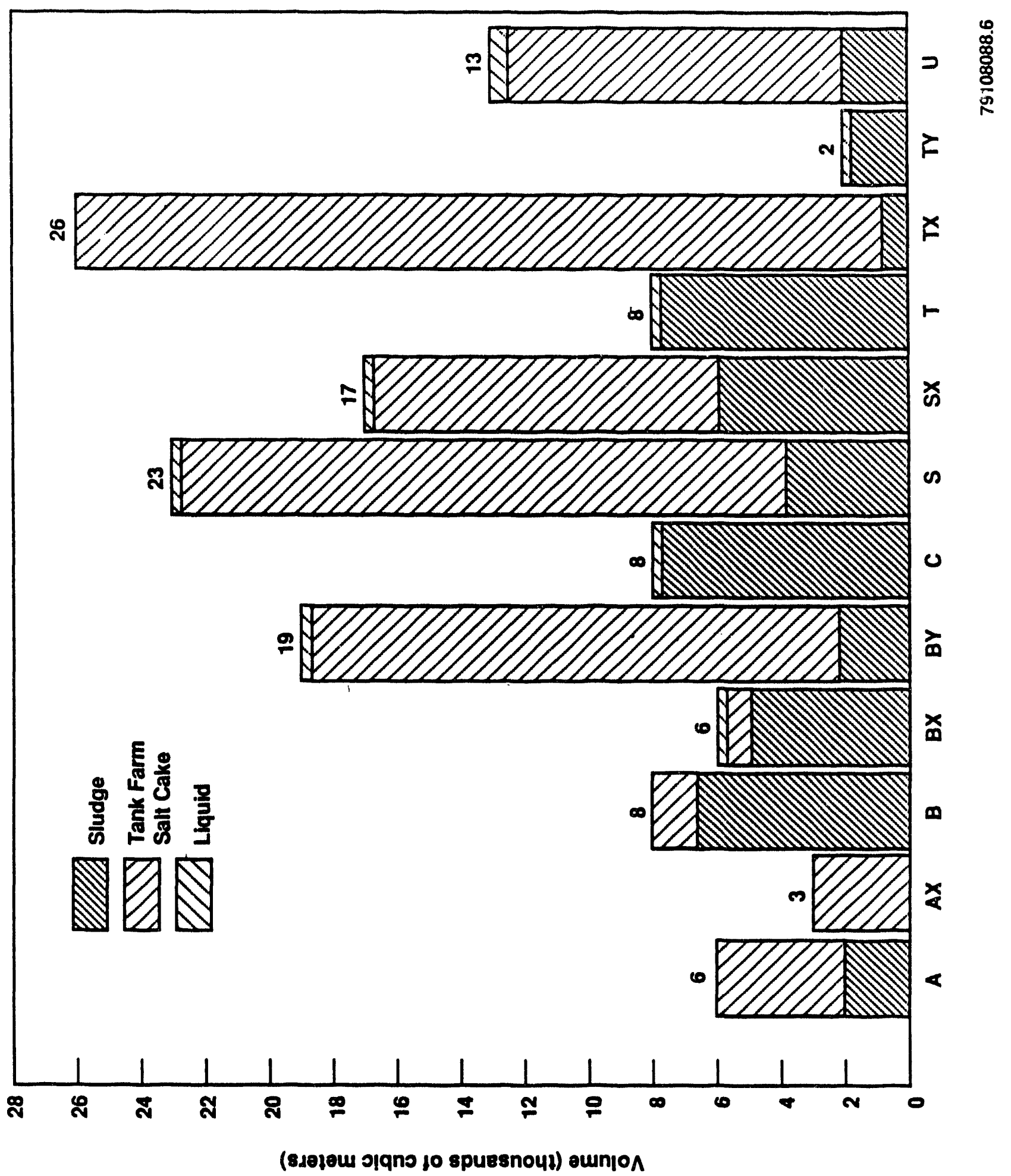

F3-5.1 


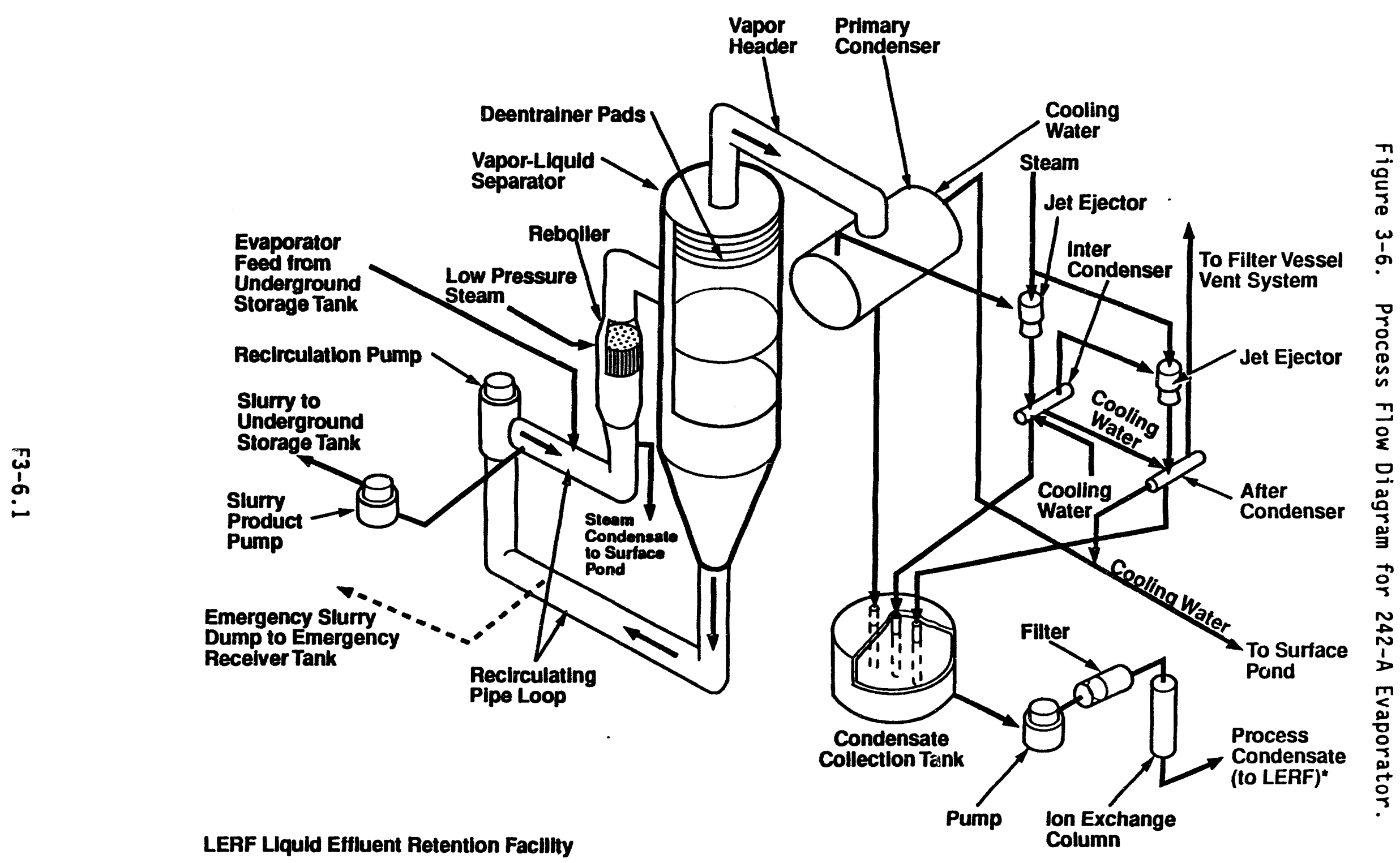

- To treatment facility after June 1995 


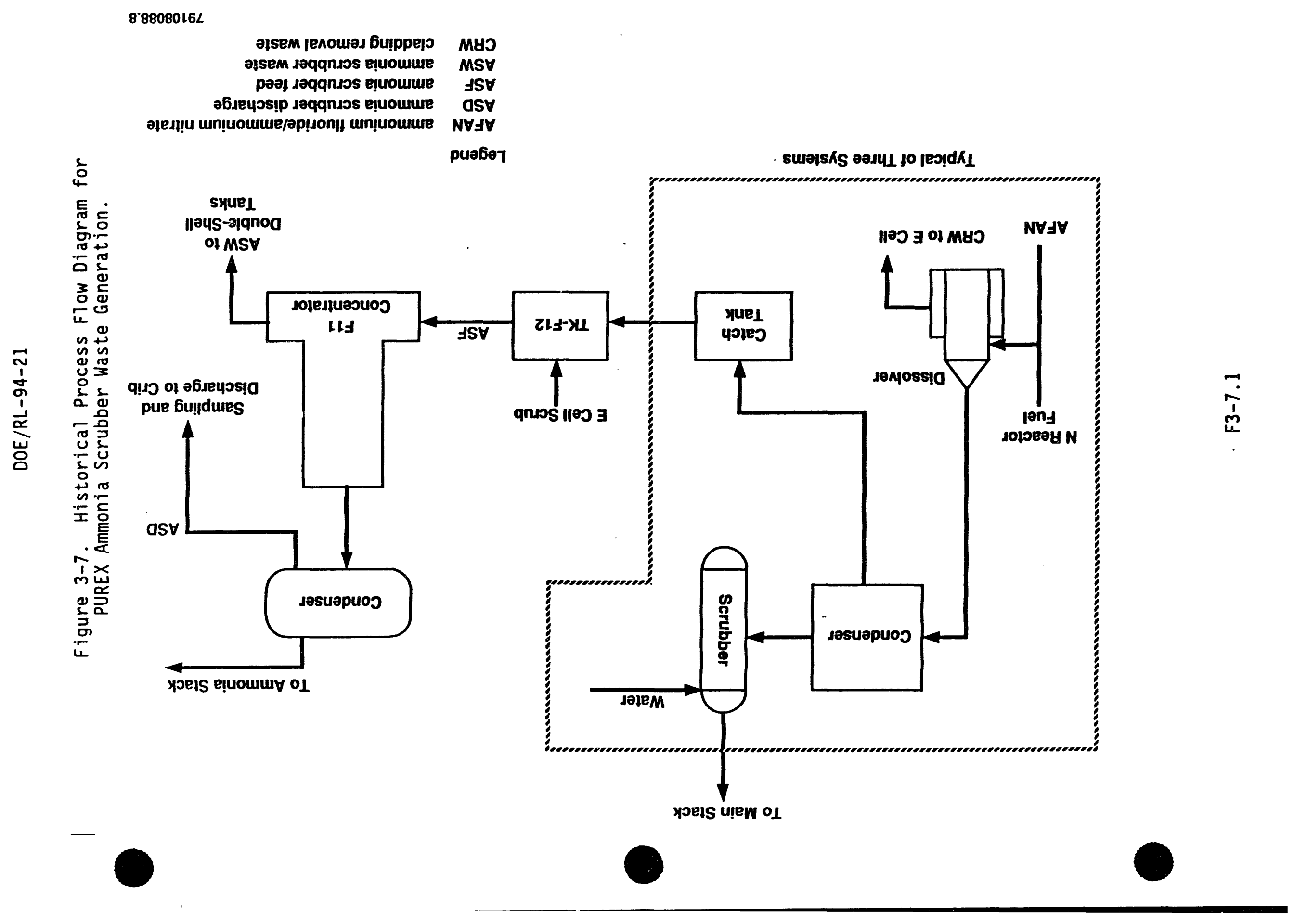


Figure 3-8. Ammonia Scrubber Waste Transfers to Double-Shell Tanks.

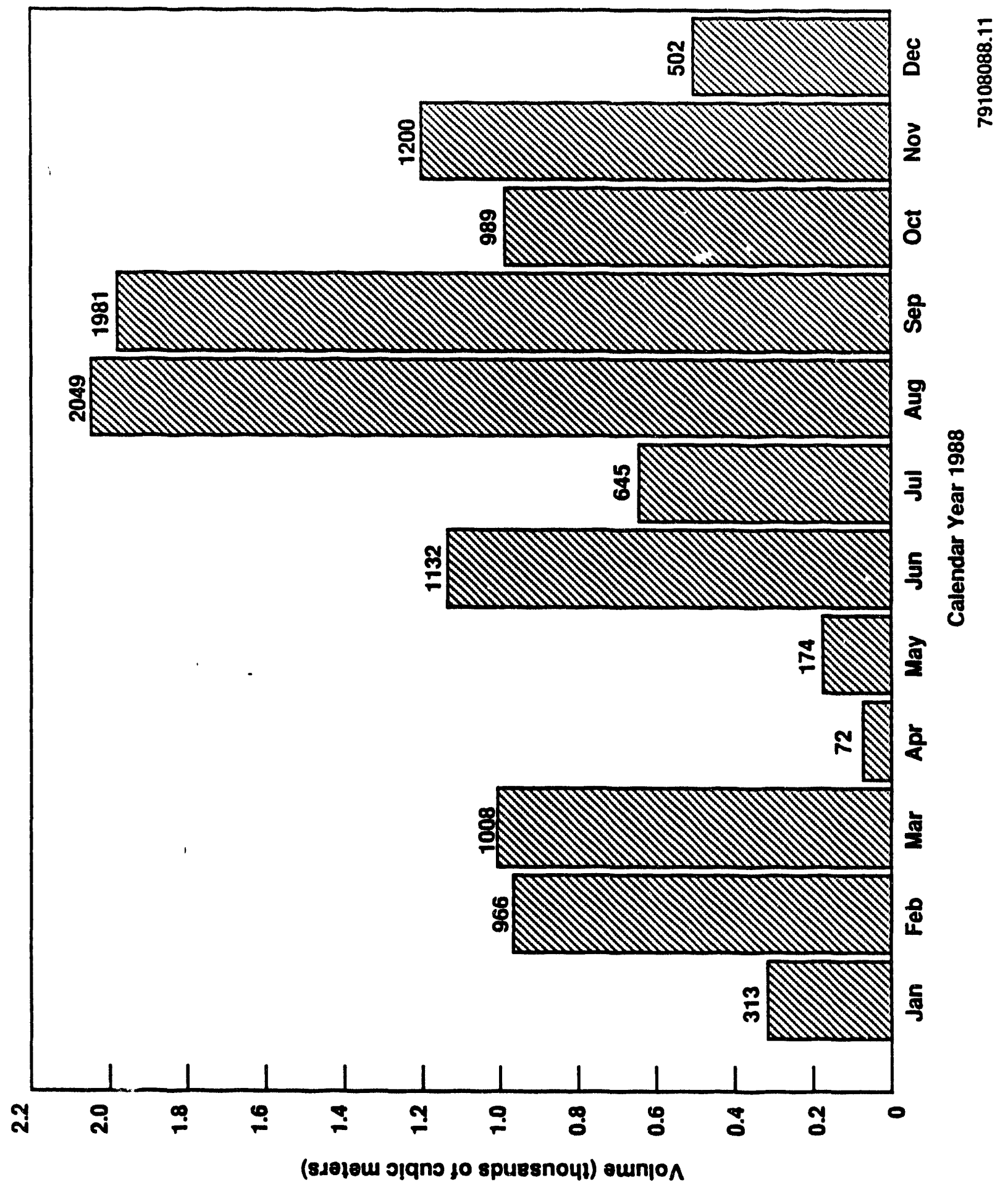




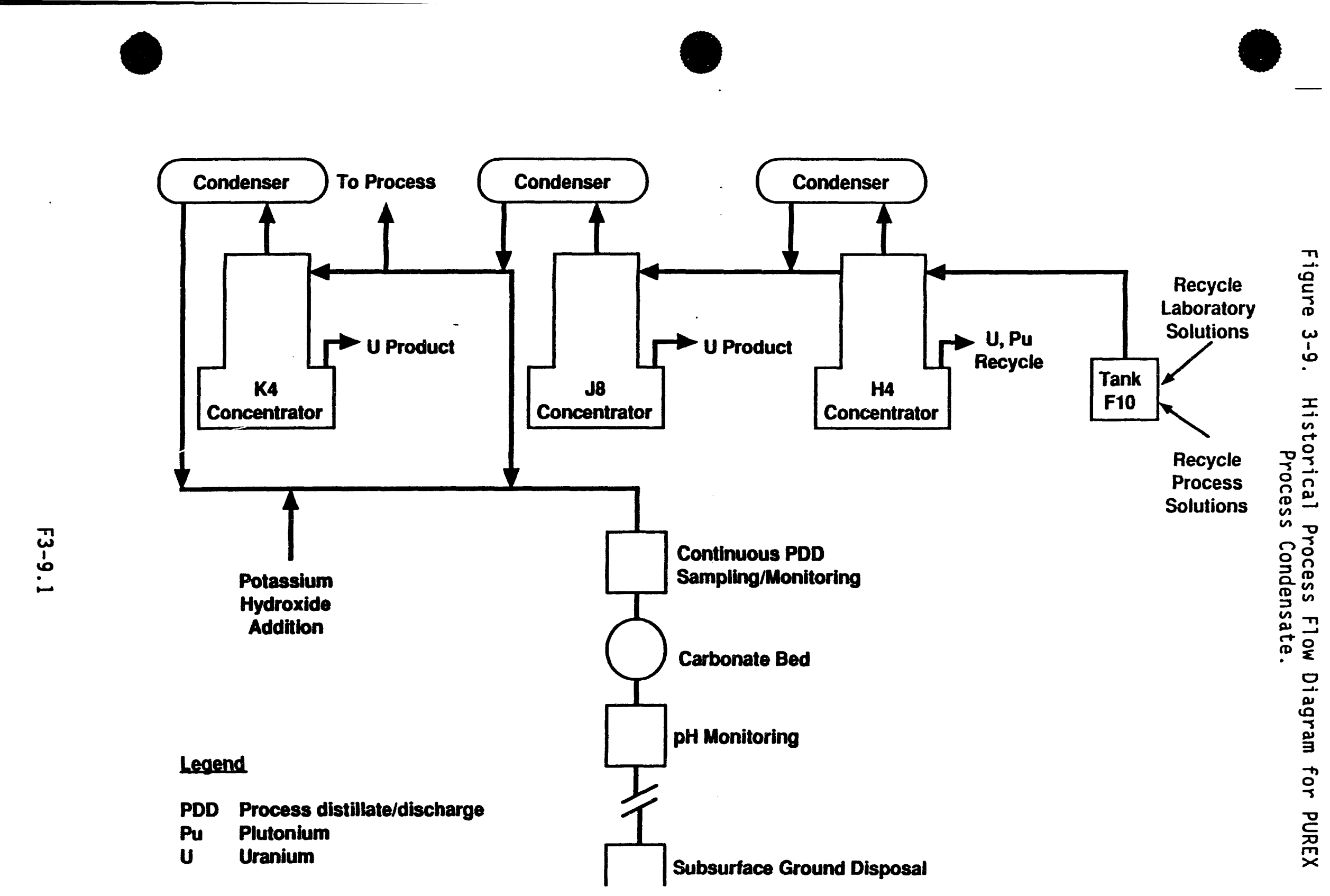




\section{Feed and Processing Equipment}

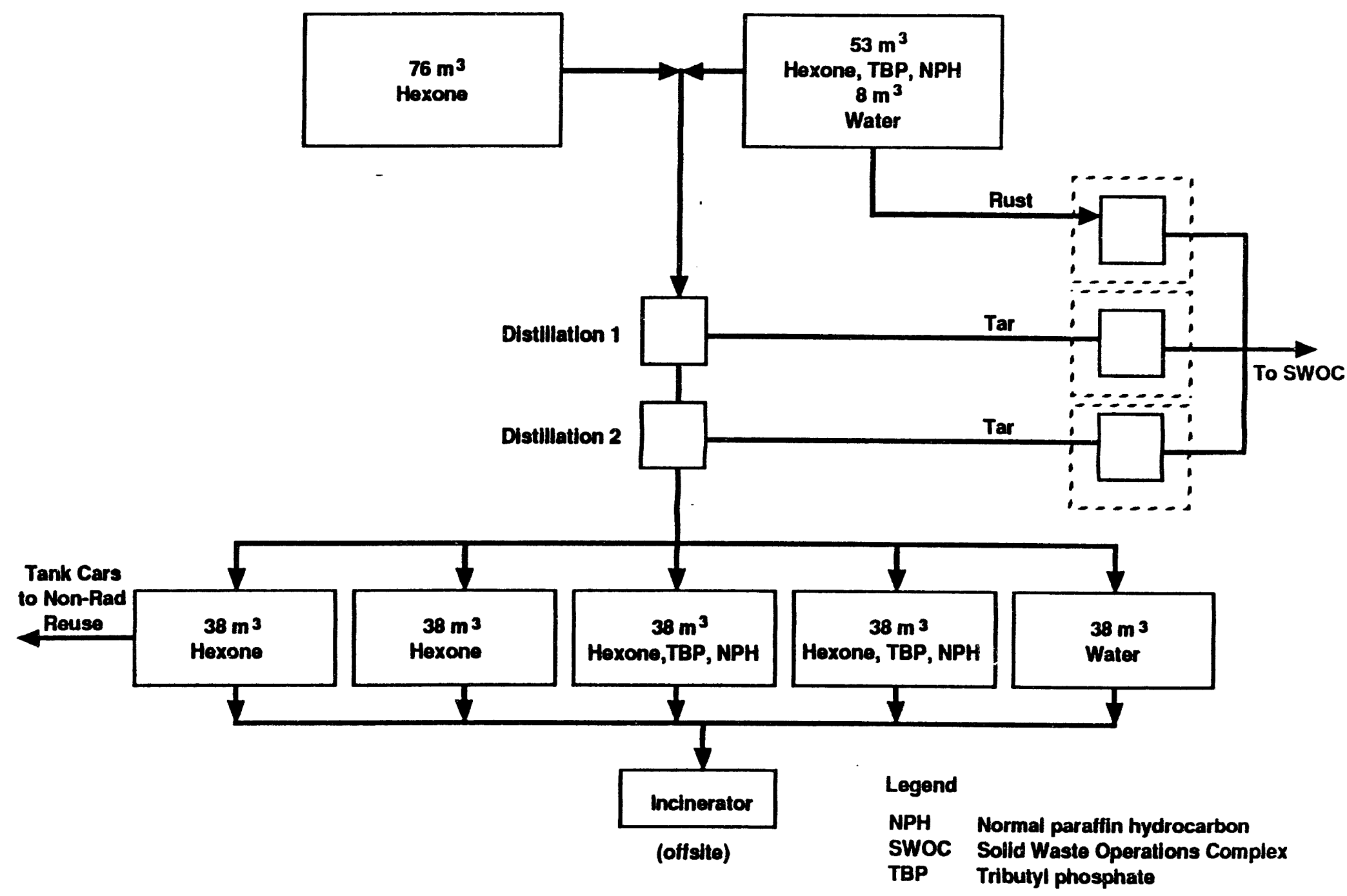


Figure 3-11. Plan View of Existing and Planned 200 West Area Facilities.
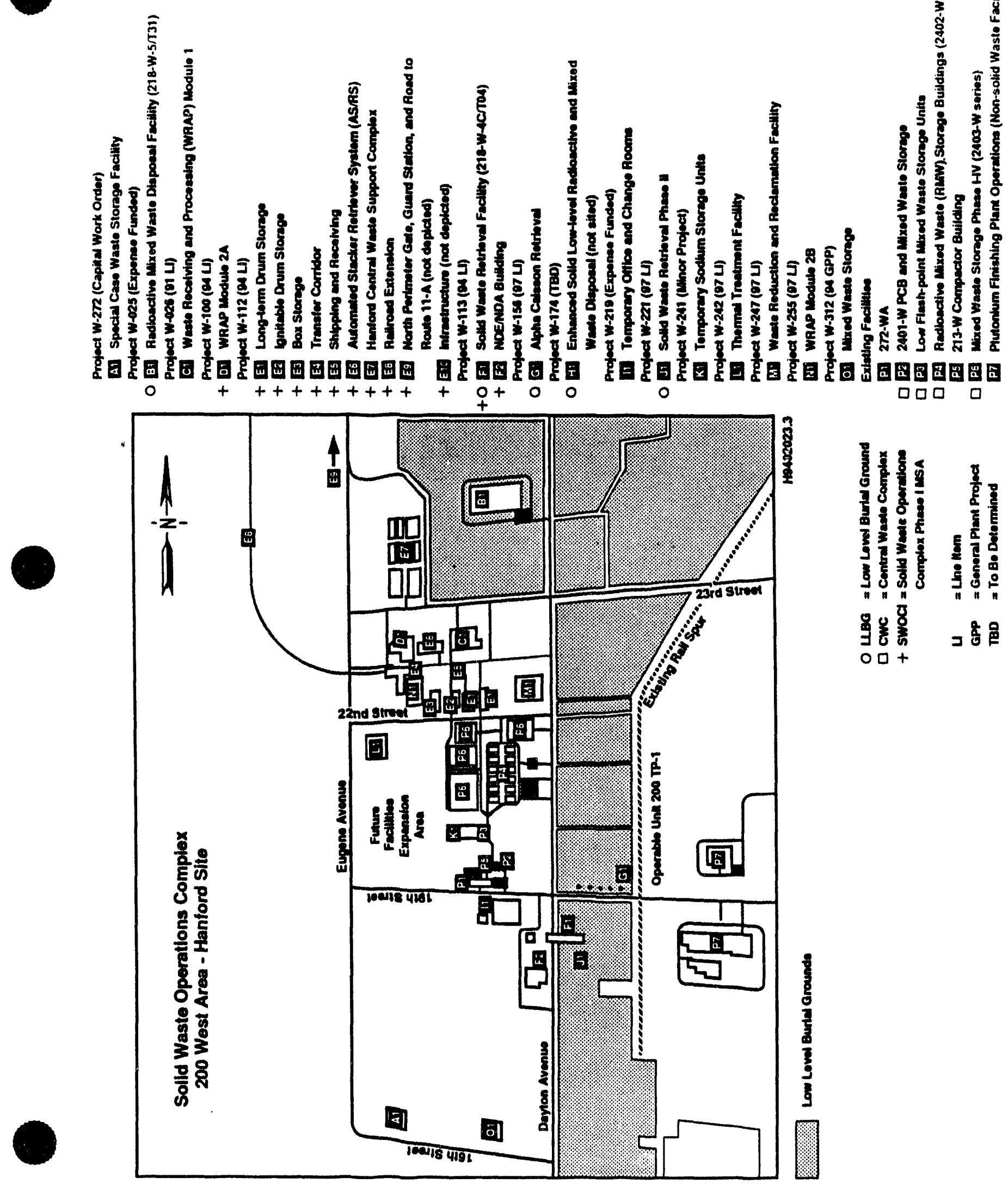

F3-11.1 
Figure 3-12. Process Flow Diagram for Proposed Waste Receiving and Processing Facility Module 2.

Grout Equipment

Cleanout

\begin{tabular}{c}
$\begin{array}{c}\text { Contaminated } \\
\text { Liquid }\end{array}$ \\
\hline
\end{tabular}

Clovebox'

Coll Washdown
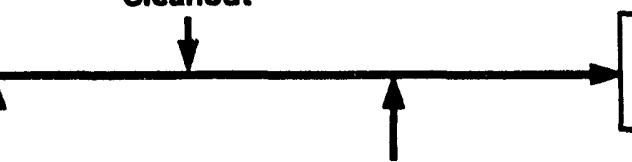

From Sludoe

Protreatmont

Particulate/Sludge Wasto

183-H Basin

Effluent Treatmone

Process Wasto

Ion Exchange Resins

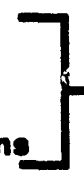

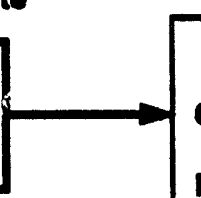

Roein and Studge Conditioning and Protreatment
Excess to

Liquid Effluent

Treatment

Liquid
Treatment
System

Facility
System

Recycle for

Grout Makoup

\section{Solldification}

To Diaposal

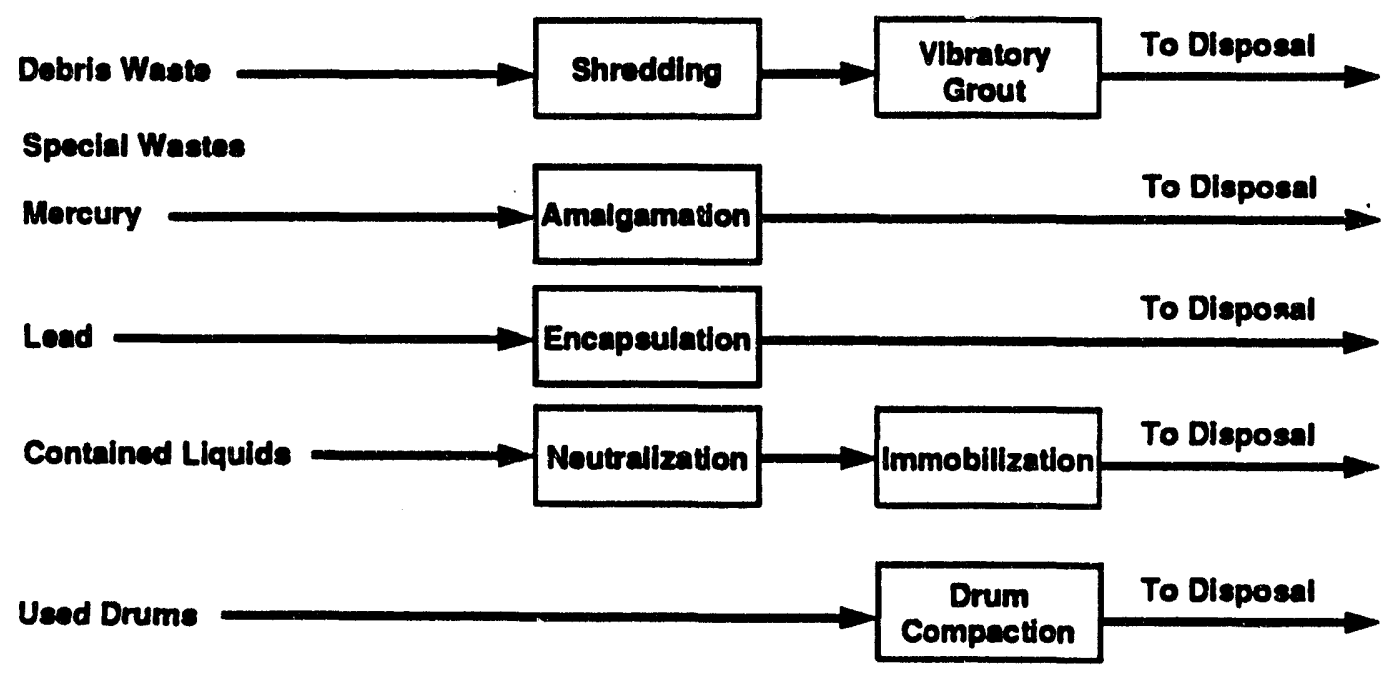

H0402023.1 


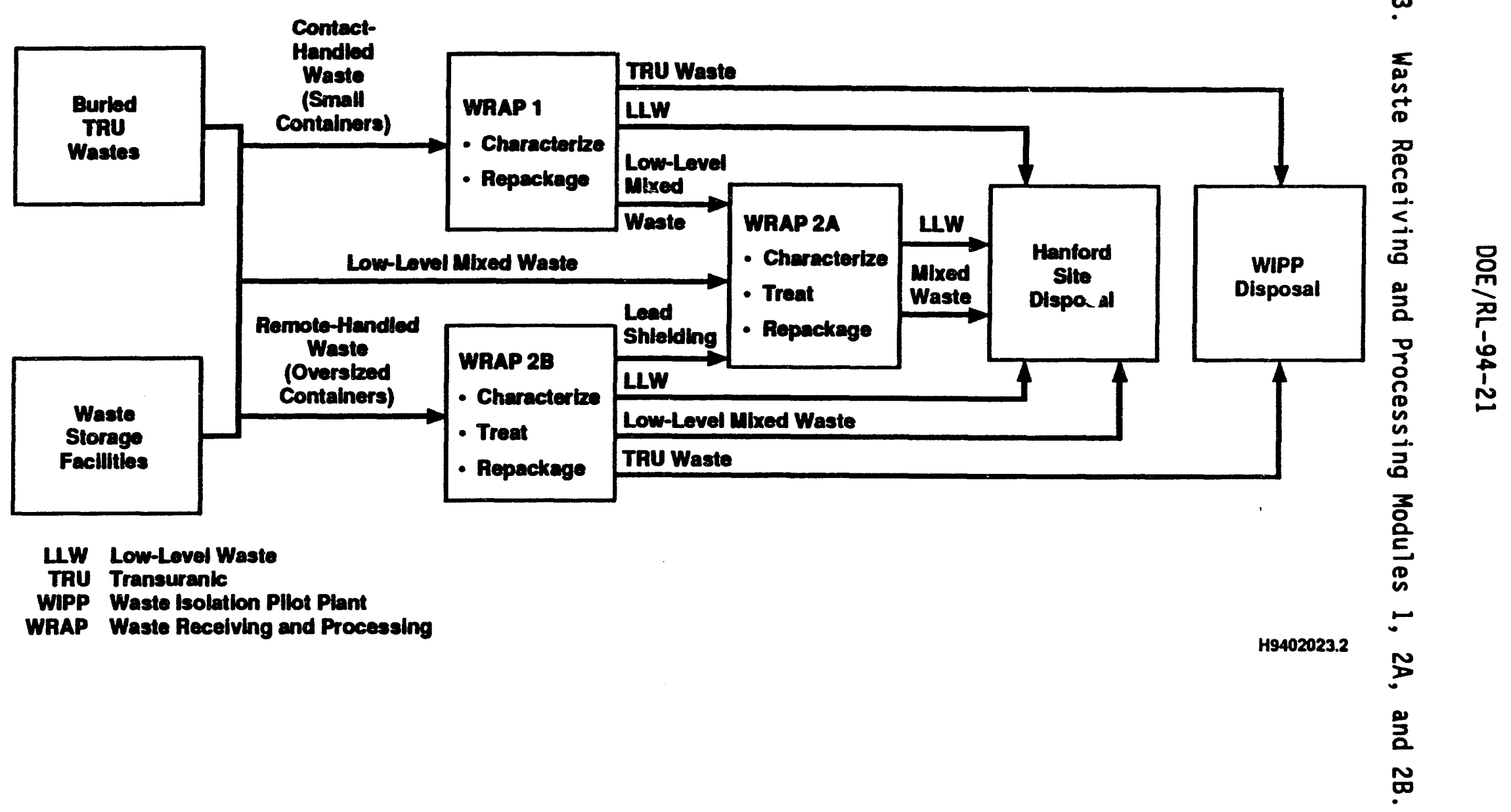


Figure 3-14. Typical Configuration of Retrievable Storage Unit for Contact-Handled Waste.

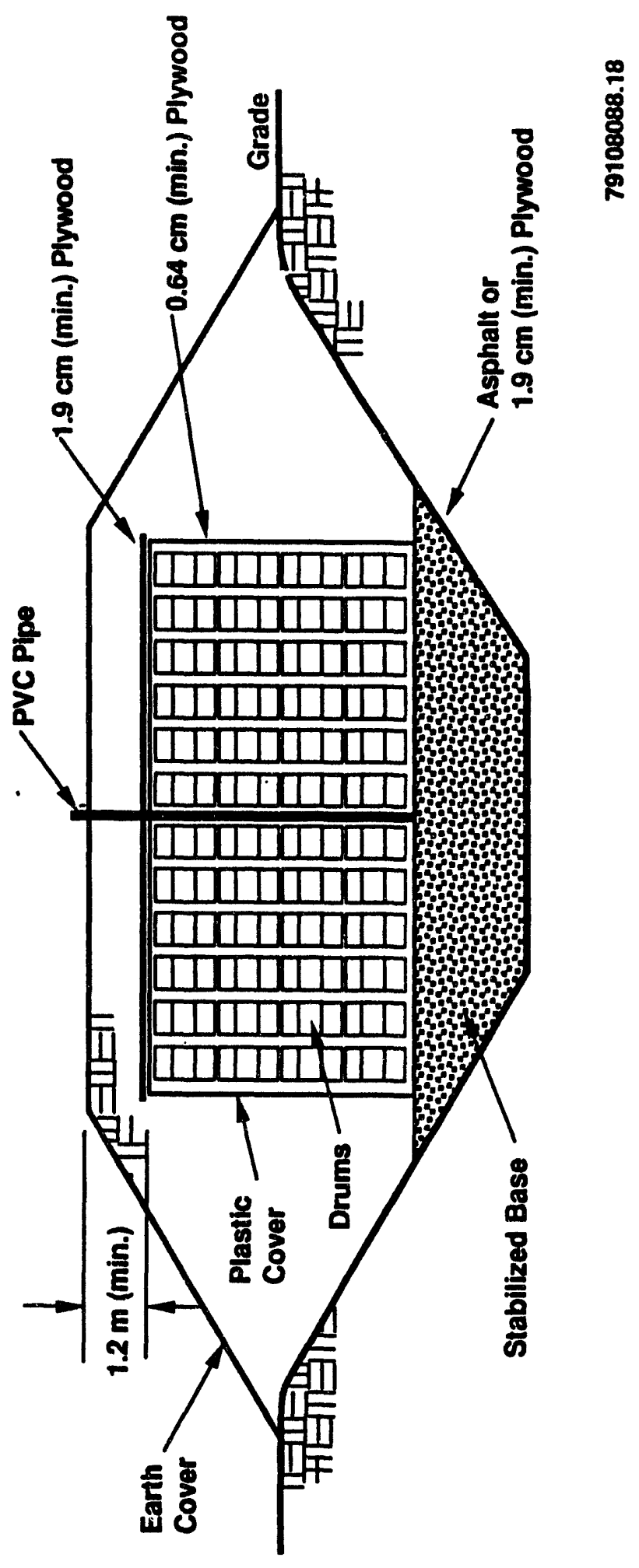


Figure 3-15. Typical Configuration of a Retrievable Storage Unit for Remote-Handled Waste.

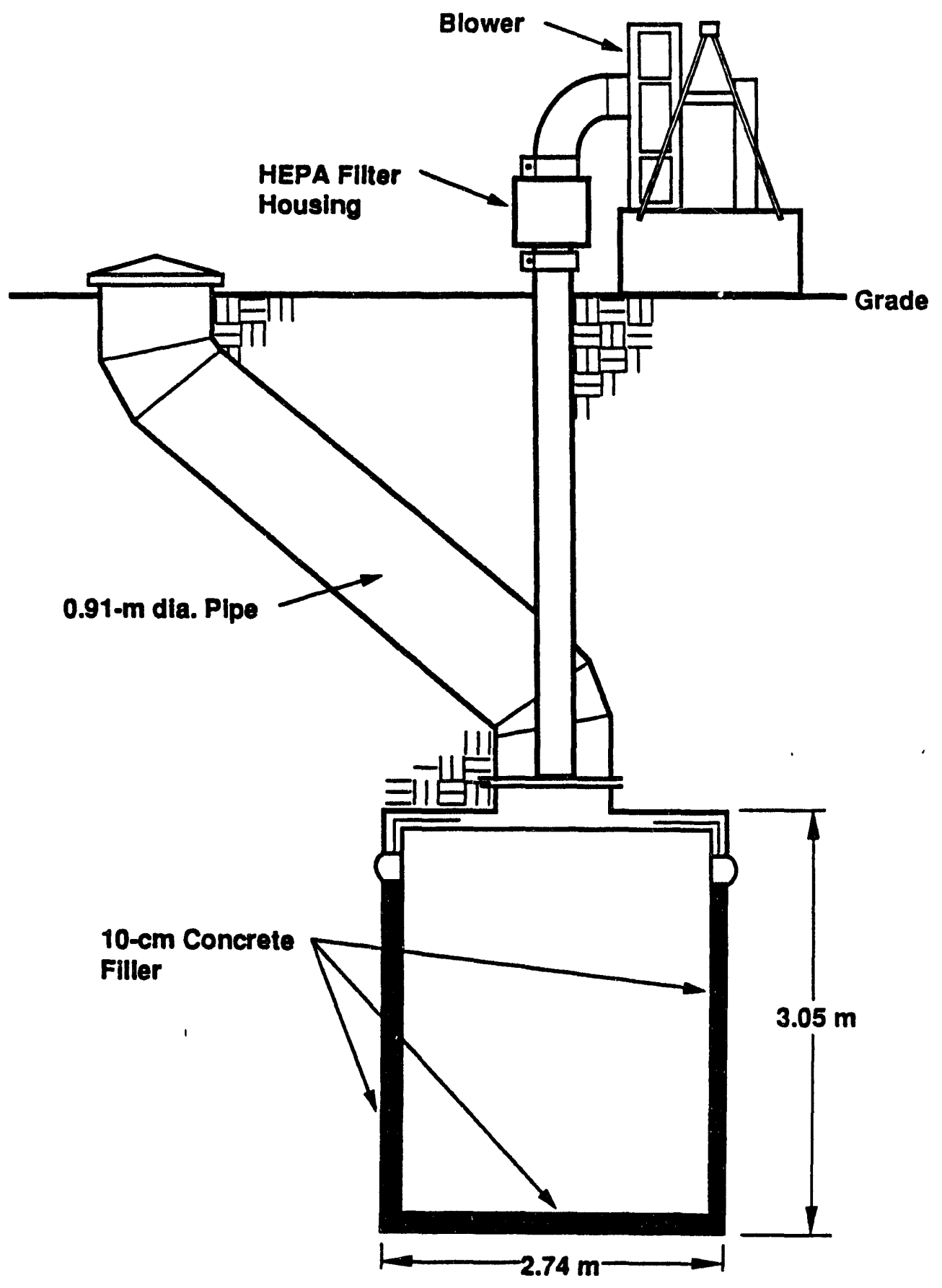

HEPA High-efficlency particulate air

79108088.19 
Figure 3-16. Transuranic Storage and Assay Facility Floor Plan.
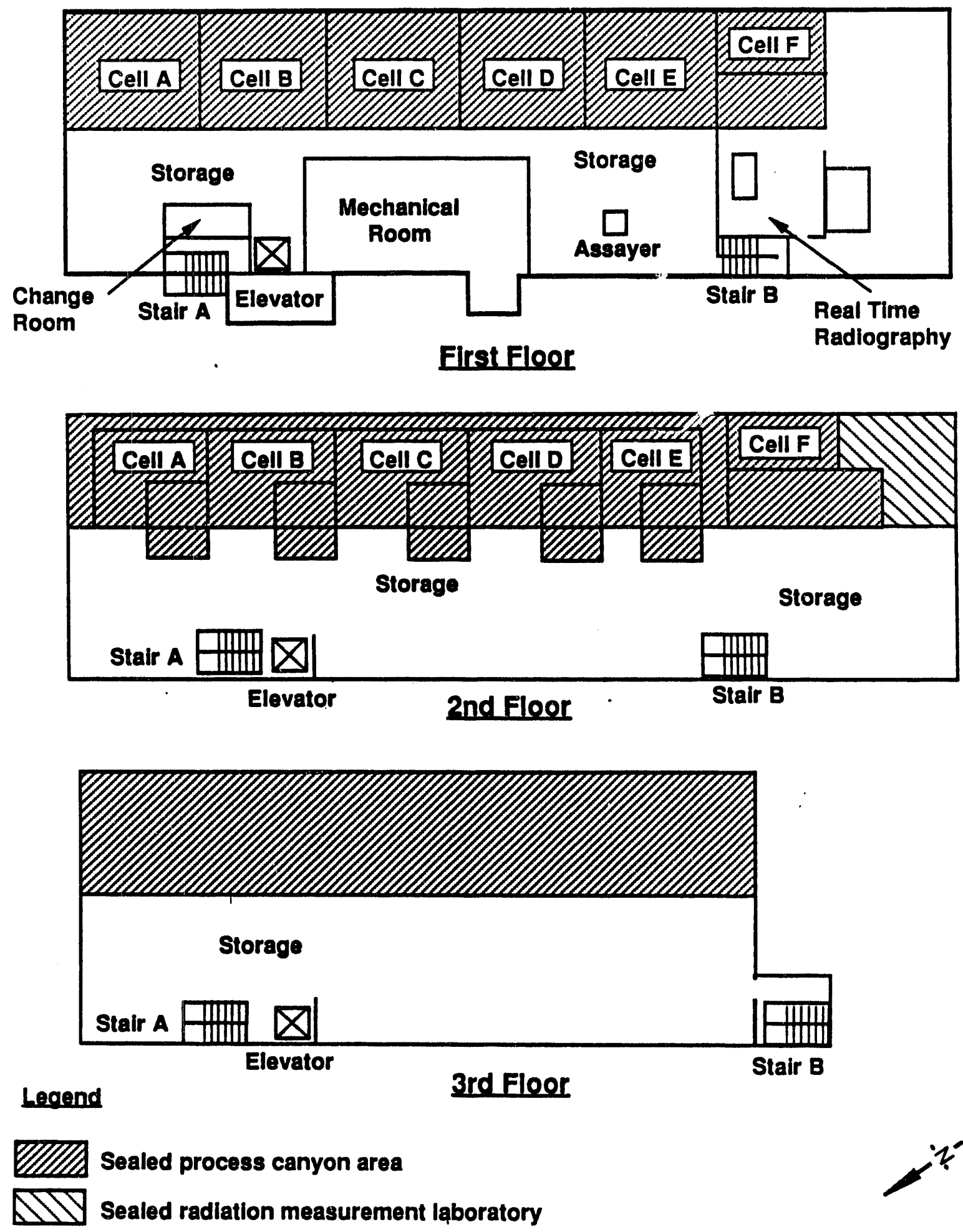

79108088.20 


\subsection{REFERENCES}

\subsection{DOCUMENTS}

Adams, M. R., L. Jensen, and W. W. Schulz, 1986, Preliminary Assessment of the TRAC Model Predictor of Key Radionuclides, RHO-RE-EV-89-P, Rockwe 11 Hanford Operations, Richland, Washington.

Boomer, et. al, 1991, Systems Engineering Study for the Closure of the SingleShell Tanks," WHC-EP-0405, Draft A, Volumes 1 through 6, Westinghouse Hanford Company, Richland, Washington.

DOE, 1987, Final Environmental Impact Statement - Disposal of Hanford Defense High-Level, Transuranic and Tank Wastes, DOE/EIS-0013, Vol. 1,

U.S. Department of Energy, Washington, D.C.

DOE, 1990, National Report on Prohibited Wastes and Treatment Options, U.S. Department of Energy, Washington, D.C.

Ecology, EPA, and DOE, 1992, Hanford Federal Facility Agreement and Consent Order, Vol. 1 and 2, as updated by the fourth amendment dated January 25, 1994, Washington State Department of Ecology, U.S. Environmental Protection Agency, and U.S. Department of Energy, Olympia, Washington.

EPA, 1983a, Guidelines and Specifications for Preparing Quality Assurance Program Plans, QAMS-004/80, U.S. Environmental Protection Agency, Washington, D.C.

EPA, 1983b, Interim Guidance and Specifications for Preparing Quality Assurance Project Plans, QAMS-005/80, U.S. Environmental Protection Agency, Washington, D.C.

EPA, 1986, Test Methods for the Evaluation of Solid Waste: Physical/Chemical Methods, SW-846, 3rd ed., U.S. Environmental Protection Agency, Washington, D.C.

RHO, 1985, Hanford Defense Waste Disposal Alternatives: Engineering Support Data for the Hanford Defense Waste Environmental Impact Statement, RHO-RE-ST-3OP, Rockwell Hanford Operations, Richland, Washington.

RL, 1989a, Hanford Site Dangerous Waste Part A Permit Application, DOE/RL-88-21, Vol. 1-3, U.S. Department of Energy, Richland Operations Office, Richiand, Washington.

RL, 1989b, Single-Shell Tank Systen Closure/Corrective Action Work Plan, DOE/RL-89-16, Draft A, U.S. Department of Energy, Richland Operations Office, Richiand, Washington.

RL, 1990a, Grout Treatment Facilicy Dangerous Waste Permit Application, DOE/RL 88-27, Rev. 1, U.S. Department of Energy, Richland Operations Office, Richland, Washington. 
RL, 1991a, Double-Shell Tank System Dangerous Waste Permit Applicntion, DOE/RL-90-39, U.S. Department of Energy, Richland Operations Office, Richl and, Washington.

RL, 1991b, 1991 Hanford Site Annual Waste Reduction Report, DOE/RL-92-25, U.S. Department of Energy, Richland Operations Office, Richland, Washington.

RL, 1991C, 183-H Solar Evaporation Basins Closure/Postclosure Plan, DOE/RL-88-04, Rev. 3, U.S. Department of Energy, Richland Operations Office, Richl and, Washington.

RL, 1993a, Waste Analysis Plan for Confirmation or Completion of Tank Farms Backlog Waste Designations, DOE/RL-93-70, U.S. Department of En-rgy, Richland Operations Office, Richland, Washington.

Sasaki, L. M., 1990, Waste Characterization Plan for the Hanford Site SingleShell Tanks, WHC-EP-0210, RCV. 2, Westinghouse Hanford Company, Richiand, Washington.

Weiss, R. L., 1986, TY Tank Farm Waste Characterization Data, RHO-WM-TI-1P, Rockwell Hanford Operations, Richland, Washington.

WHC, 1989a, Characterization Plan for Stored Contact Handled Transuranic Waste, WHC-EP-0223, Westinghouse Hanford Company, Richland, Washington.

WHC, 1989b, Sampling Plan for Retrievably Stored Contact Handled Transuranic Waste at Hanford, WHC-EP-0226, Westinghouse Hanford Company, Richland, Washington.

WHC, 1990a, Double-Shell Tank Waste Disposal Integration Plan, WHC-EP-0229, Rev. 1, Westinghouse Hanford Company, Richland, Washington.

WHC, 1990b, Engineering Study for Waste Receiving and Processing (WRAP) Facility, Module 2, WHC-SD-W100-ES-001, Rev. 0, Westinghouse Hanford Company, Richland, Washington.

WHC, 1990c, Functional Requirements Baseline for the Closure of Single-Shell Tanks-Draft, WHC-EP-0338, Westinghouse Hanford Company, Richland, Washington.

WHC, 1990d, Hanford Site LDR RMW Stream Data Package, WHC-MR-0224, Westinghouse Hanford Company, Richland, Washington.

WHC, 1990e, A History of the 200 Area Tank Farms, WHC-MR-0132, Westinghouse Hanford Company, Richland, Washington.

WHC, 1990f, PUREX Plant Ammonia Scrubber Distillate Stream-Specific Report, Addendum 14-Final, WHC-EP-0342-14, Westinghouse Hanford Company, Richland, Washington.

WHC, 1990g, PUREX Plant Process Condensate Stream-Specific Report, WHC-EP-0342-12, WHC, Richl and, Washington. 
WHC, 1990h, Single-Shell Tank Waste Retrieval Study, WHC-EP-0352, Westinghouse Hanford Company, Richland, Washington.

WHC, 1990i, Solid Waste Management Program P7an, WHC-EP-0363, Rev 1, Westinghouse Hanford Company, Richland, Washington.

WHC, 1990j, 242-A Evaporator Process Condensate Stream-Specific Report, Addendum 15-Fina1, WHC-EP-0342-15, Nestinghouse Hanford Company, Richland, Washington.

WHC. 1991c, Tank Wastes Discharged Directly to Soil at the Hanford Site, WHC-MR-0227, Westinghouse Hanford Company, Richland, Washington.

WHC, 1991f, 1991 Borehole Completion Package for the Low-Level Burial Grounds, WHC-SD-EN-DP-044, Rev. 0, Westinghouse Hanford Company, Rich 1 and, Washington.

WHC, 1992c, Tank Waste Technical Options Report, WHC-EP-0616, Westinghouse Hanford Compiany, Richland, Washington.

WHC, 1992d, 1992 Borehole Completion Package for the Low-Level Burial Grounds, WHC-SD-EN-DP-049, Rev. 0, Westinghouse Hanford Company, Richland, Washington.

WHC, 1993a, Alternative Pretreatment Technologies for Removal of Transuranic Elements from Selected Hanford Site Wastes, WHC-EP-0577, Westinghouse Hanford Company, Richland, Washington.

WHC, 1993b, Single-Shell Tank Waste Characterization Project and Safety Analysis Project, Core 34, 35, and 36, Tank 241-C-112, SD-WMD-P026ENG Rev. 0, Westinghouse Hanford Company, Richland, Washington.

WHC, 1993c, Single-Shell Tank Waste Characterization, Tank C110 Core 37, 38, and 39 Data Package, SD-WMD-P027ENG Rev. 0, Westinghouse Hanford Company, Richland, Washington.

WHC, 1993d, Annual Report of Tank Waste Treatability, WHC-EP-0365-03, Westinghouse Hanford Company, Richland, Washington.

WHC, 1993e, Tank Farm Surveillance and Waste Status Summary Report for December 1993, WHC-EP-0182-69, Westinghouse Hanford Company, Richland, Washington.

WHC, 1993f, Hanford Site Solid Waste Acceptance Criteria, WHC-EP-0063-4, Westinghouse Hanford Company, Richland, Washington.

\subsection{CODE OF FEDERAL REGULATIONS AND FEDERAL REGISTER}

40 CFR 265, "Interim Status Standards for Owners and Operators of Hazardous Waste Treatment, Storage, and Disposal Facilities," Title 40, Code of Federal Regulations, Part 265, as amended, U.S. Environmental Protection Agency, Washington, D.C. 
40 CFR 268, "Land Disposal Restrictions," Title 40, Code of Federal Regulations, Part 268, as amended, U.S. Environmental Protection Agency, Washington, D.C.

40 CFR 761, "Polychlorinated Biphenyls (PCB) Manufacturing, Processing, Distribution in Commerce, and Use Prohibitions," Title 40, Code of Federal Regulations, Part 761, as amended, U.S. Environmental Protection Agency, Washington, D.C.

55 FR 22520, et seq., "Land Disposal Restrictions for Third Third Scheduled Wastes; Final Rule," Federal Register, Vol. 55, No. 106, Sec. III.A.8.C. (2), P. 22627, June 1, 1990, U.S. Environmental Protection Agency, Washington, D.C.

58 FR 29800, et seq., "Land Disposal Restriction for Ignitable and Corrosive Characteristic Wastes Whose Treatment Standards Were Vacated, "Federal Register, Vo1. 58, No. 98, p. 29860, May 24, 1993, U.S. Environmental Protection Agency, Washington, D.C.

\subsection{FEDERAL AND STATE ACTS}

Atomic Energy Act of 1954, as amended, 42 USC 2011.

Comprehensive Environmental Response, Compensation, and Liability Act of 1980, as amended, 42 USC 9601 et seq.

Federal Facility Compliance Act of 1992, need citation.

National Environmental Policy Act of 1969, as amended, 42 USC 4321, et seq.

Resource Conservation and Recovery Act of 1976, as amended, 42 USC 6901, et seq.

State of Washington Hazardous Waste Management Act of 1976, as amended, Revised Code of Washington, Chapter 70.105, 01 ympia, Washington.

Toxic Substances Control Act of 1976, 15 USC 2601, et seq.

\subsection{HASHINGTON STATE REGULATIONS}

Ecology, Technical Information Memorandum 86-3, Treatment by Generators (revised July, 1993), Hazardous Waste and Toxics Reduction Program, Technical Assistance and Policy Sections, Washington State Department of Ecology, 0lympia, Washington.

WAC, 1990, Dangerous Waste Regulations, Washington Administrative Code 173-303, Washington State Department of Ecology, O1ympia, Washington. (WAC 173-303-140 covers land disposal restrictions.) 
DOE/RL-94-21

INVENTORY DATA CORRELATION

A- i

\section{APPENDIX A}


DOE/RL-94-21

This page intentionally left blank. 
In response to EPA's comments on the 1993 Land Disposal Restrictions Report, this crosswalk is provided that correlates inventory data among a number of reports. These reports are the following:

- Waste Isolation Pilot Plant No-Migration Variance Petition

- November 1991 Case-by-Case Extension Application

- April 1993 Interim Mixed Waste Inventory Report, required by the Federal Facility Compliance Act of 1992.

The approach in this correlation is to use the Interim Mixed Waste Inventory Report as the basis for comparisons. Thus, the organization of this correlation is the following. First, the data in the Interim Mixed Waste Inventory Report is summarized. Next, a correlation will be presented with the data in the Waste Isolation Pilot Plant No-Migration Variance Petition. This will be followed by a comparison with the Case-by-Case Extension Application.

The purpose of this correlation is to show the consistency of inventory data. Any inconsistencies are explained.

\section{A1.0 INTERIM MIXED WASTE INVENTORY REPORT SUMMARY}

A summary of data presented in the Interim Mixed Waste Inventory Report for the Hanford Site is presented in Tables A-1, A-2, and A-3. Tank waste not subject to land disposal restriction (LDR) is single-shell tank (SST) waste. The solid wastes in storage that are not subject to LDR are the following:

- State-only regulated waste

- EPA regulated waste that have only total carbon-organics waste codes.

These wastes are not subject to LDR because relevant LDR standards have not been promulgated.

The data in the Interim Mixed Waste Inventory Report exclude polychlorinated biphenyl (PCB) - TSCA regulated waste.

\section{A2.0 COMPARISON TO WASTE ISOLATION PILOT}

PLANT NO-NIGRATION VARIANCE PETITION

Only transuranic waste managed by Solid Waste Programs is planned to be shipped to Waste Isolation Pilot Plant, and is thereby. covered in their No-Migration Variance Petition. Tank waste that meets the definition of transuranic waste is planned to be pretreated as necessary and vitrified at the Hanford Waste Vitrification Plant. The canisters containing vitrified 
transuranic tank waste are candidates for disposal at the Waste Isolation Pilot Plant. Thus, these canisters were not covered in the Waste Isolation Pilot Plant No-Migration Variance Petition.

The comparison of mixed transuranic waste inventories and projections, between the Interim Mixed Waste Inventory Report and the Waste Isolation Pilot Plant No-Migration Variance Petition, is presented in Table A-4. The existing transuranic waste inventory in the No-Migration Variance is expected to be less than the inventory in the Interim Mixed Waste Inventory Report, and this is reflected in Table A-4. This is because the inventories in the No-Migration Variance Petition only cover the period to $12 / 31 / 88$.

Projected quantities of mixed transuranic waste in the No-Migration Variance Petition are much less than the Interim Mixed Waste Inventory Report. Transuranic mixed waste descriptions are presented in Tables 3-8 and 3-9 of the No-Migration Variance Petition. These are presented in Table A-5, along with the waste descriptions based on Hanford Site records of the types of waste reported in the Interim Mixed Waste Inventory Report. As the iformation in Table A-5 indicates, the types of waste for the Hanford Site included in the No-Migration Variance Petition includes a small fraction of the wastes that have been actually received at the Hanford Site. The data in the No-Migration Variance Petition does not capture much of the debris generated from maintenance and cleanup activities. 
Table A-1. Interim Mixed Waste Inventory Report Volume Summary".

\begin{tabular}{|l|l|l|c|c|c|}
\hline \multicolumn{1}{|c|}{ Waste } & Type & $\begin{array}{c}\text { Reg. } \\
\text { Auth. }\end{array}$ & $\begin{array}{c}\text { Not subject } \\
\text { to LDR, } \mathrm{m}^{3}\end{array}$ & $\begin{array}{c}\text { Subject to } \\
\text { LDR, } \mathrm{m}^{3}\end{array}$ & Tota1, $\mathrm{m}^{3}$ \\
\hline Tank Waste & HLW & EPA & $138,600.000$ & $7,352.000$ & $145,952.000$ \\
\hline Tank Waste & MTRU & EPA & 0.000 & $2,767.000$ & $2,767.000$ \\
\hline Tank Waste & MLLW & EPA & 0.000 & $81,872.000$ & $81,872.000$ \\
\hline Wastewater & MLLW & EPA & 0.000 & 0.000 & 0.000 \\
\hline 183-H waste & MLLW & EPA & 0.000 & $2,309.600$ & $2,309.600$ \\
\hline Solid Waste & MTRU & EPA & 0.000 & 166.150 & 166.150 \\
\hline Solid Waste & MTRU & State & 2.100 & 0.000 & 2.100 \\
\hline Solid Waste & MLLW & EPA & 16.600 & 505.680 & 522.280 \\
\hline Solid Waste & MLLW & State & 89.180 & 0.000 & 89.180 \\
\hline $\begin{array}{l}\text { Other Solid } \\
\text { Waste }\end{array}$ & MLLW & EPA & 0.000 & 9.641 & 9.641 \\
\hline Total & & & $138,707.880$ & $94,982.071$ & $233,689.951$ \\
\hline
\end{tabular}

Inventories are cumulative through 12/31/91.

bSol id waste is defined as waste managed by Westinghouse Hanford Company, Solid Waste Programs. The 183-H Basin waste is al so managed by Solid Waste Programs, but because of its relative magnitude of inventory the data are tabulated separately. Inventories of solid waste are cumulative from mid-1987, reflecting the date of the Byproduct Rulemaking. "Other solid waste" refers to waste not managed by Solid Waste Programs-the waste is in permitted storage at other locations on the Hanford Site. ${ }^{c} \mathrm{HLW}=$ High-level Waste, MTRU = Mixed Transuranic Waste, MLLW = Mixed Low-level Waste. All HLW is mixed waste, per DOE Order 5820.2A, "Radioactive Waste Management." 
Table A-2. Interim Mixed Waste Inventory Report Mass Summary".

\begin{tabular}{|l|c|c|c|c|c|}
\hline \multicolumn{1}{|c|}{ Waste } & Type & $\begin{array}{c}\text { Reg. } \\
\text { Auth. }\end{array}$ & $\begin{array}{c}\text { Not subject } \\
\text { to LDR, kg }\end{array}$ & $\begin{array}{c}\text { Subject to } \\
\text { LDR, } \mathrm{kg}\end{array}$ & Total, kg \\
\hline Tank waste & HLW & EPA & $248,000,000$ & $8,822,000$ & $256,822,000$ \\
\hline Tank waste & MTRU & EPA & 0 & $3,876,200$ & $3,876,200$ \\
\hline Tank waste & MLLW & EPA & 600 & $98,267,400$ & $98,268,000$ \\
\hline Wastewater & MLLW & EPA & 0 & 0 & 0 \\
\hline 183-H waste & MLLW & EPA & 0 & $2,309,630$ & $2,309,630$ \\
\hline Solid waste & MTRU & EPA & 0 & 166,030 & 166,030 \\
\hline Solid waste & MTRU & State & 2,100 & 0 & 2,100 \\
\hline Solid waste & MLLW & EPA & 16,590 & 513,420 & 530,010 \\
\hline Solid waste & MLLW & State & 91,585 & 0 & 91,585 \\
\hline $\begin{array}{l}\text { Other solid } \\
\text { waste }\end{array}$ & MLLW & EPA & 0 & 15,638 & 15,638 \\
\hline Total & & & $248,110,875$ & $113,970,318$ & $362,081,193$ \\
\hline
\end{tabular}

The footnotes in Table A-1 also apply to Table A-2.

Table A-3. Interim Mixed Waste Inventory Report

Projections Summary".

\begin{tabular}{|l|c|c|c|c|}
\hline \multicolumn{1}{|c|}{ Waste } & Type & $\begin{array}{c}\text { Reg. } \\
\text { Auth. }\end{array}$ & $\begin{array}{c}5 \text { Year Projerted } \\
\text { Generation, } \mathbf{m}^{36}\end{array}$ & $\begin{array}{c}\text { 5 Year Projected } \\
\text { Generation, } \text { kg }^{6}\end{array}$ \\
\hline Tank waste & HLW & EPA & 0.000 & 0 \\
\hline Tank waste & MTRU & EPA & 241.000 & 361,500 \\
\hline Tank waste & MLLW & EPA & $110,119.000$ & $121,745,600$ \\
\hline Wastewater & MLLW & EPA & $114,600.000$ & $114,600,000$ \\
\hline 183-H waste & MLLW & EPA & 310.800 & 310,800 \\
\hline Solid waste & MTRU & EPA & $1,297.560$ & $1,297,440$ \\
\hline Solid waste & MTRU & State & 14.100 & 14,070 \\
\hline Solid waste & MLLW & EPA & $10,266.810$ & $10,381,725$ \\
\hline Solid waste & MLLW & State & $1,024.700$ & $1,057,640$ \\
\hline Other solid waste & MLLW & EPA & 0.872 & 667 \\
\hline Total & & & $237,874.842$ & $249,769,442$ \\
\hline
\end{tabular}

The footnotes in Table A-1 apply to Table A-3.

The five-year projection was required by statute, the data reported in the Interim Mixed Waste Inventory Report actually covers a six-year period, from $1 / 1 / 92$ to $12 / 31 / 97$. 
Table A-4. Data Comparison between the Interim Mixed Waste Inventory Report and the Waste Isolation Pilot Plant No-Migration Variance Petition.

\begin{tabular}{|c|c|c|c|}
\hline \multicolumn{2}{|c|}{ Existing inventory } & \multicolumn{2}{c|}{$\begin{array}{c}\text { Annual average projection } \\
\mathrm{m}^{3} / \mathrm{yr}\end{array}$} \\
\hline IMWIR (a) & $\begin{array}{c}\text { WIPP No Mig } \\
\text { Petition Var. }\end{array}$ & IMWIR (a) & $\begin{array}{c}\text { WIPP No Mig } \\
\text { Petition Var. }\end{array}$ \\
\hline mid 87 to $12 / 31 / 91$ & $\begin{array}{c}\text { through } \\
12 / 31 / 88\end{array}$ & $\begin{array}{c}1 / 1 / 92 \text { to } \\
12 / 31 / 97\end{array}$ & 1988 to 2013 \\
\hline 168.25 & 25 & 218.61 & 11.12 \\
\hline
\end{tabular}

Table A-5. Mixed Transuranic Waste Description Comparison.

\begin{tabular}{|c|c|c|c|}
\hline \multicolumn{2}{|c|}{$\begin{array}{l}\text { Waste Isolation Pilot Plant } \\
\text { No-Migration Variance } \\
\text { Petition }\end{array}$} & \multicolumn{2}{|c|}{ Interim Mixed Waste Inventory Report } \\
\hline $\begin{array}{l}\text { Waste } \\
\text { description }\end{array}$ & $\begin{array}{l}\text { Chemical } \\
\text { contaminants }\end{array}$ & Waste description & $\begin{array}{l}\text { Chemical } \\
\text { contaminants }\end{array}$ \\
\hline $\begin{array}{l}\text { Organic cleanup } \\
\text { of solvent } \\
\text { extraction } \\
\text { system and } \\
\text { analytical lab } \\
\text { waste } \\
\text { Neutralized } \\
\text { rags used to } \\
\text { clean PUREX } \\
\text { and PFP } \\
\text { equipment } \\
\text { Gloves } \\
\text { containing } \\
\text { lead shielding }\end{array}$ & $\begin{array}{l}\text { Sodium } \\
\text { hydroxide }\end{array}$ & $\begin{array}{l}\text { Cleanup activities } \\
\text { Maintenance activities } \\
\text { Spent equipment } \\
\text { Miscellaneous } \\
\text { inorganic spent } \\
\text { materials } \\
\text { Contaminated soil } \\
\text { Contaminated absorbents } \\
\text { Lead acid batteries packaged } \\
\text { with soil or absorbent } \\
\text { Orained spent lead acid } \\
\text { batteries contaminated with } \\
\text { Mercury } \\
\text { Radioactive contaminated lead } \\
\text { packaged with soil and } \\
\text { various solid debris } \\
\text { Lead glass packaged with } \\
\text { various solid debris } \\
\text { Cellulose material } \\
\text { plastic } \\
\text { Rubber } \\
\text { Cloth }\end{array}$ & $\begin{array}{l}\text { Lead chromate } \\
\text { TC-metals } \\
\text { Sodium } \\
\text { hydroxide } \\
\text { Cadmium } \\
\text { Phosphoric } \\
\text { acid } \\
\text { Sulfuric acid } \\
\text { Mercury } \\
\text { Beryll ium } \\
\text { Tri-chloro- } \\
\text { ethene } \\
\text { Methylene } \\
\text { chloride } \\
\text { PCB's (with } \\
\text { other RCRA } \\
\text { contaminants } \\
\text { Lead }\end{array}$ \\
\hline
\end{tabular}

Hanford has not reported any stored remote-handled mixed transuranic waste, either as inventories or projections, in either the Interim Mixed Waste Invertory Report or the Waste Isolation Pilot Plant No-Migration Variance Fetition. 


\section{A3.0 COMPARISON TO THE CASE-BY-CASE APPLICATION}

The data comparison between the Case-by-Case Extension Application and the Interim Mixed Waste Inventory Report is presented in Table A-6. An explanation of the differences are as follows:

- Existing inventories--Most of the difference reflects actual generation in CY 1991. A minor correction was made to the inventory of SST waste. Minor corrections for the other waste categories are also incorporated into the difference.

- Projections for tank waste in the Case-by-Case Extension Application excluded residues of waste generated within tank farms. The residues were included in the Interim Mixed Waste Inventory Report. These residues are as follows:

- Evaporator Bottoms (Double-shell Slurry and Doubleshell Slurry Feed)

- Evaporator Overhead (Process Condensate, Wastewaters).

- Projections for tank waste in the Case-by-Case Extension Application were based on an average generation rate for each waste stream. Projections for tank waste in the Interim Mixed Waste Inventory Report were based on detailed 5-year projections provided by each of the generators and on planned operating campaigns of the Evaporator.

- Projections for tank waste in the Case-by-Case Extension Application were based on the programmatic assumptions applicable in November 1990. At that time, the plan called for continuation of fuel reprocessing and continued operation at the Plutonium Finishing Plant. While preparing the Interim Mixed Waste Inventory Report (Spring 1993), the plan was not to restart the fuel reprocessing plant, and to perform only necessary operations at PFP to place the facility in a safe maintenance condition.

- In the Case-by-Case Extension Application, the assumption was that retrieval of dewatered fuel fabrication siudge from the 183-H Basins had been completed. However, additional quantities of 183-H Basin waste was received in 1991.

- Projections of Solid Waste (other than 183-H Basin waste) in the Case-by-Case Extension Application was based on the annial generation of each waste stream in the 3.5-year period between mid-1987, the approximate date of the Byproduct Rulemaking, and $12 / 31 / 90$. Projections of solid waste in the Interim Mixed Waste Inventory Report were based on Westinghouse Hanford Company Solid Waste Reference Forecast. 
Table A-6. Data Comparison between Interim Mixed Waste Inventory Report and the Case-by-Case Extension Application.

\begin{tabular}{|c|c|c|c|c|c|c|}
\hline & & & Invent & ry, $\mathrm{m}^{3}$ & $\begin{array}{r}\text { Projected a } \\
\text { generat } \\
\end{array}$ & $\begin{array}{l}\text { erage annual } \\
n, m^{3} / y r\end{array}$ \\
\hline Waste & Type & $\begin{array}{l}\text { Reg. } \\
\text { auth. }\end{array}$ & $\begin{array}{c}\text { Case-by-Case } \\
\text { Extension } \\
\text { Application } \\
12 / 31 / 90\end{array}$ & $\begin{array}{c}\text { Interim Mixed } \\
\text { Waste } \\
\text { Inventory } \\
\text { Report, } \\
12 / 31 / 91 \\
\end{array}$ & $\begin{array}{l}\text { Case-by-Case } \\
\text { Extension } \\
\text { Application }\end{array}$ & $\begin{array}{c}\text { Interim Mixed } \\
\text { Waste } \\
\text { Inventory } \\
\text { Report }\end{array}$ \\
\hline $\begin{array}{l}\text { Single-shell tank } \\
\text { waste }\end{array}$ & & & $139,500.000$ & $138,600.000$ & 0.000 & 0.000 \\
\hline $\begin{array}{l}\text { Double-shell tank } \\
\text { waste }\end{array}$ & HLH & EPA & $7,237.000$ & $7,352.000$ & 0.000 & 0.000 \\
\hline $\begin{array}{l}\text { Double-shell tank } \\
\text { waste }\end{array}$ & MTRU & EPA & $2,767.000$ & $2,767.000$ & 829.000 & 40.167 \\
\hline $\begin{array}{l}\text { Double-shell tank } \\
\text { waste }\end{array}$ & MLLW & EPA & $78,231.000$ & $81,872.000$ & $13,931.000$ & $18,353.167$ \\
\hline Wastewater & MLLW & EPA & 0.000 & 0.000 & 0.000 & $19,100.000$ \\
\hline 183-H waste & MLLW & EPA & $1,781.030$ & $2,309.600$ & 0.000 & 51.800 \\
\hline Solid waste & MTRU & EPA & 160.570 & 166.150 & 45.877 & 216.260 \\
\hline Solid waste & MTRU & State & 2.100 & 2.100 & 0.600 & 2.350 \\
\hline Sol id waste & MLLW & EPA & 441.900 & 522.280 & 126.257 & $1,711.135$ \\
\hline Solid waste & MLLW & State & 72.160 & 89.180 & 20.617 & 170.783 \\
\hline Other solid waste & MLLW & EPA & -- & 9.641 & -- & 0.145 \\
\hline Solid waste & MTRU & TSCA & 1.470 & (a) & 0.420 & (a) \\
\hline Solid waste & MLLW & TSCA & 43.430 & (a) & 12.409 & (a) \\
\hline
\end{tabular}

(a) The Interim Mixed Waste inventory Report did not include TSCA regulated PCBs. 
This page intentionally left blank. 
DOE/RL-94-21

DISTRIBUTION

number of Copies

OFFSITE

13

Washington State Department

of Ecology

OTympia, Washington 98504

G. Anderson (5) PV-11

U.S. Environmental Protection Agency

Richland, Washington

D. R. Sherwood (5) B5-01

U.S. Department of Energy-Headquarters Washington. D.C.

$\begin{array}{ll}\text { G. Deleon (2) } & \text { EM-322 } \\ \text { P. Bubar } & \text { EM-323 }\end{array}$

ONSITE

11

U.S. Department of Energy. Richland Operations Office

R. F. Guercia (10) R3-80

Public Reading Room H2-53

1 Bechtel Hanford. Incorporated

R. H. Wyer H4-79

1

Pacific Northwest Laboratory

Technical Files K1-11

72 Westinghouse Hanford Comoany

B. A. Austin B2-35

J. W. Badden H6-22

D. G. Black (25) H6-20

R. C. Bowman H6-24

B. J. Broomfield N3-13

F. T. Calapristi B2-35

F. M. Coony H6-07

C. Defigh-Price R2-31

B. G. Erlandson H6-21

L. A. Garner R2-86

K. A. Gasper L6-29 


\section{DISTRIBUTION (cont)}

Number of Copies

ONSITE

Westinghouse Hanford Comoany (cont)

C. J. Geier

W. T. Gretsinger (3) R2-86

R. D. Gustavson R1-51

D. L. Hage1

H5-33

C. S. Haller

R2-12

P. H. Jacobsen

J. R. Kirkendall

N3-13

O. S. Kramer

H4-21

R. J. Landon

B2-35

M. M. Lane

G. J. LeBaron

P. J. Mackey

M. M. McCarthy (5)

D. S. Merry

P. C. Miller

H6-21

S6-19

S6-19

B3-06

N3-13

H4-21

R. J. Murkowski

N2-04

R. D. Pierce

R4-01

0 . R. Rasmussen

T3-04

R. C. Roal

R1-51

R. J. Roberts

F. A. Ruck, III

G6-08

N3-13

H6-23

J. 0. Skolrud

H6-20

J. N. Strode

E. A. Weakley

EPIC (2)

Central files

Document Processing and Distribution (2)

Information Release

Administration (3)

RI-51

L6-26

H6-08

L8-04

(2) $\quad$ $8-15$

A3-36 

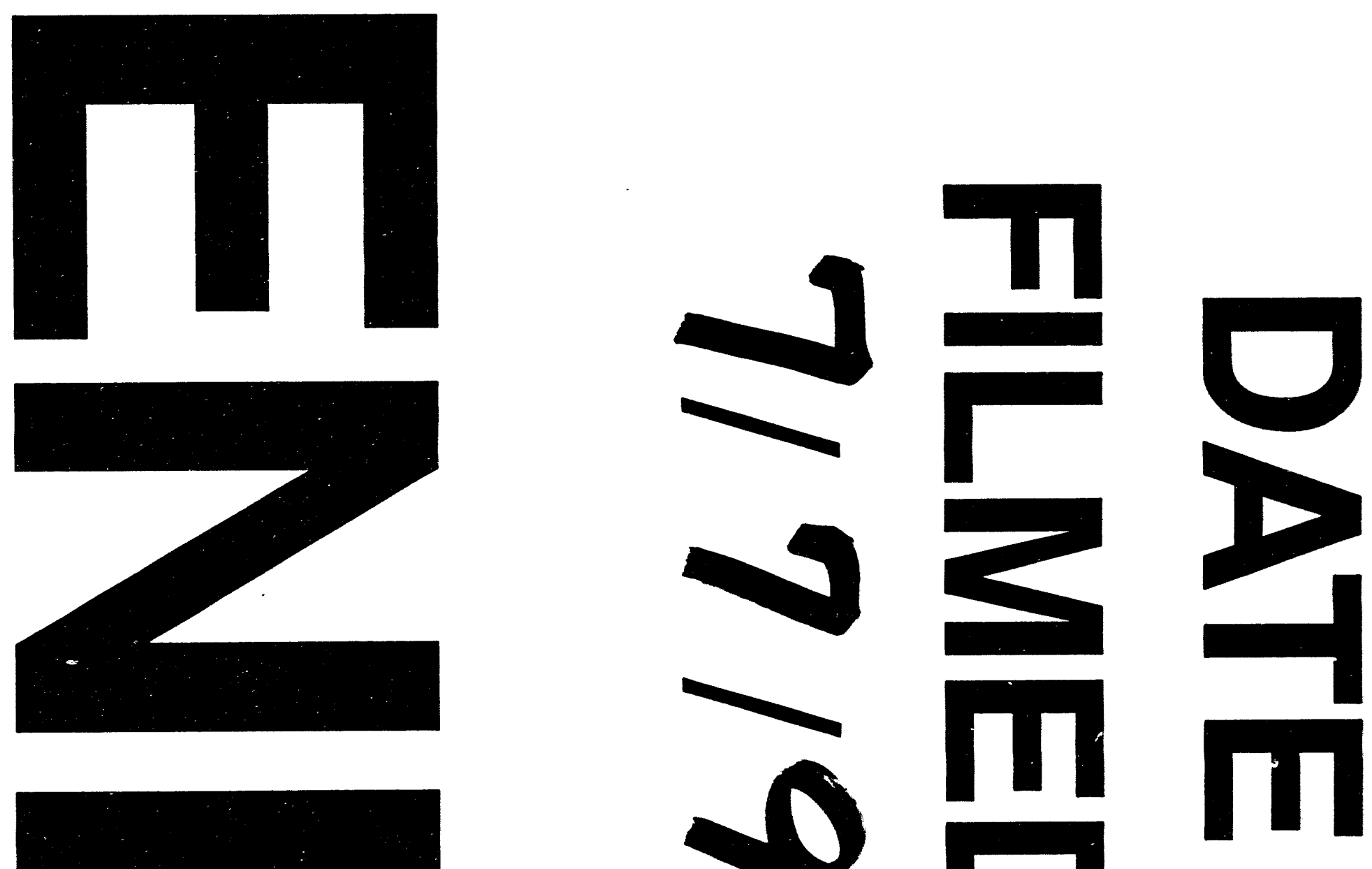

$\Sigma$
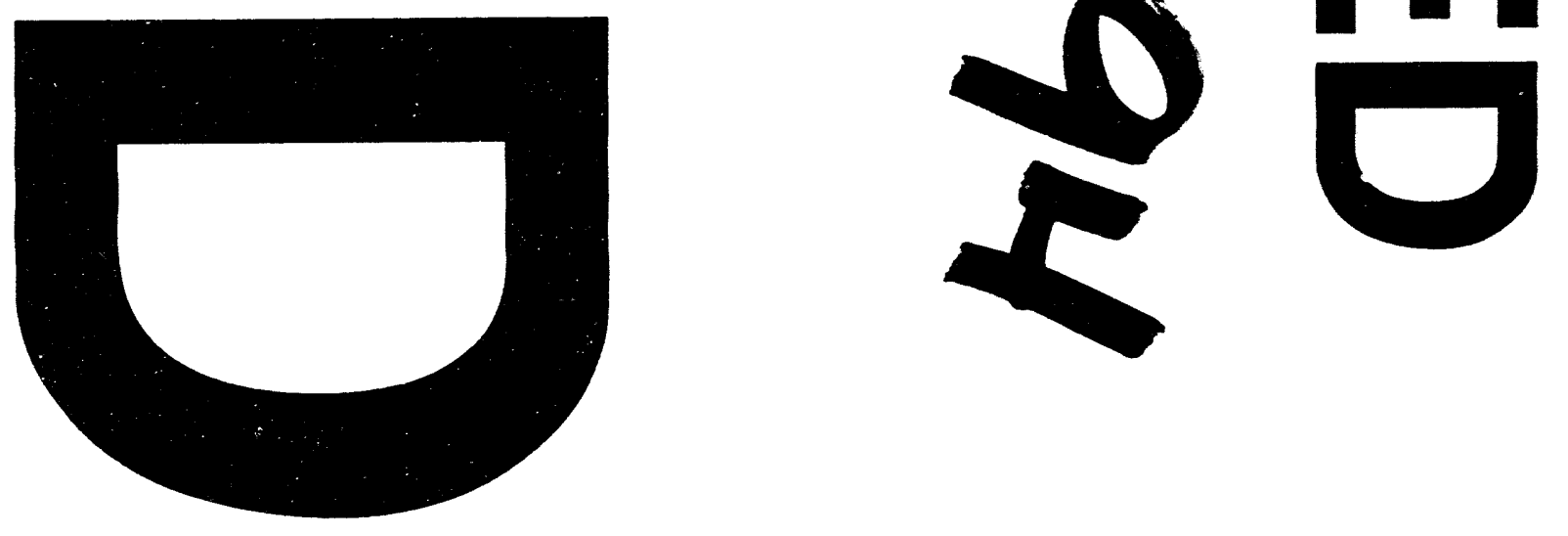
